\title{
EFFECTS OF SPIN-FLIP SCATTERING ON THE
}

\section{DYNAMICS OF THE SUPERCONDUCTING ORDER}

\author{
PARAMETER
}

F. E. ASPEN

\section{Solid State and Low Temperature Physics Group}

SCHOOL OF PHYSICS AND ASTRONOMY

เก

JUNE 1980 


\section{DISCLAIMER}

This report was prepared as an account of work sponsored by an agency of the United States Government. Neither the United States Government nor any agency Thereof, nor any of their employees, makes any warranty, express or implied, or assumes any legal liability or responsibility for the accuracy, completeness, or usefulness of any information, apparatus, product, or process disclosed, or represents that its use would not infringe privately owned rights. Reference herein to any specific commercial product, process, or service by trade name, trademark, manufacturer, or otherwise does not necessarily constitute or imply its endorsement, recommendation, or favoring by the United States Government or any agency thereof. The views and opinions of authors expressed herein do not necessarily state or reflect those of the United States Government or any agency thereof. 


\section{DISCLAIMER}

Portions of this document may be illegible in electronic image products. Images are produced from the best available original document. 


\author{
EFFECTS OF SPIN-FLIP SCATTERING \\ ON THE \\ DYNAMICS OF THE SUPERCONDUCTING ORDER PARAMETER
}

A THESIS

SUBMITTED TO THE FACULTY OF THE GRADUATE SCHOOL OF THE UNIVERSITY OF MINNESOTA

\title{
By
}

Frank Eugene Aspen

\author{
IN PARTIAL FULFILLMENT OF THE REQUIREMENTS \\ FOR THE DEGREE OF \\ DOCTOR OF PHILOSOPHY
}

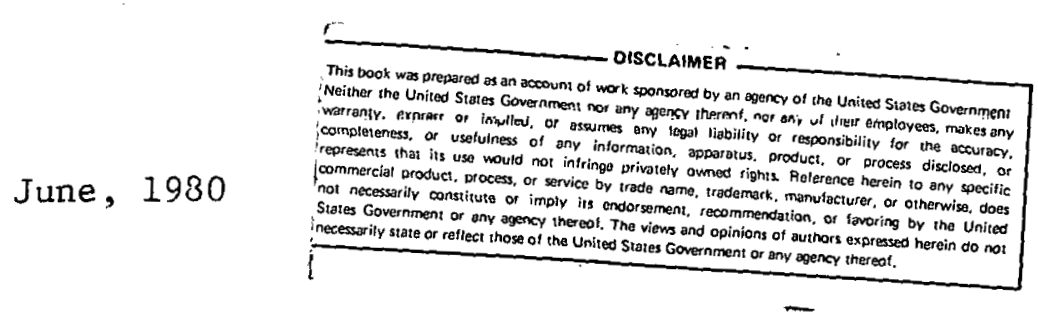




\author{
Abstract \\ EFFECTS OF SPIN-FLIP SCATTERING \\ ON THE \\ DYNAMICS OF THE SUPERCONDUCTING ORDER PARAMETER
}

Measurements of the imaginary part of the pair-field susceptibility $\chi "$ have been carried out on dirty-limit Al-Er alloy films. Aluminum films with erbium concentrations of up to one atomic percent were incorporated as the low $\mathrm{T}_{c}$ film of an asymetric Josephson. junction. The excess current $I_{\text {ex }}$ due to pair tunneling was measured as a function of the bias voltage $\mathrm{V}$ and of the magnetic field $\mathrm{H}$, applied in the plane of the junction, at temperatures within $20 \%$ of the critical temperature $\mathrm{T}_{c}$. Comparison to theory was facilitated by noting that $\chi^{\prime \prime}(\omega, \mathrm{k}) \propto \mathrm{I}_{\mathrm{ex}}(\mathrm{V}, \mathrm{H})$ where the frequency $\omega$ and wavenumber $k$ are related to $V$ and $H$ via the Josephson relations.

These experiments represent the first measurements of $\chi^{\prime \prime}(\omega, k)$ as a function of the pair-breaking parameter $\rho$. Spin-flip'scattering from the tir impurities resulted in values of $\rho$ up to 0.1 .

Several important results were obtained from these experiments. At temperatures above $\mathrm{T}_{c}$ it was verified that the diffusive timedependent Ginzburg-Landau equation for order-parameter fiuctuations is valid in the presence of pair-breaking. The diffusion rate was found to be reduced by the pair-breaking to a value which is in quantitative agreement with theory.

The characteristic frequencies of $X$ " were determined from the 
measurements of $I_{e x}$ below $T_{c}$ by fitting to a functional form which exhibits the essential features of the various theories. These frequencies were compared in detail to values determined from the theories of Orbach and Entin-Wohlman, Dinter, and Schön and Ambegaokar. . The "Carlson-Goldman mode" was found to be overdamped for large values of $\rho$. This is consistent with theoretical calculations of the transverse mode in the presence of finite pair-breaking. Quantitative comparison could not be made because theoretical calculations have been carried out only for magnetic fields smaller than that which was necessary to perform the experiment.

A peak in the excess current near the gap voltage was determined to be due to a longitudinal mode in the order parameter fluctuations and not due to single particle tunneling as had been reported in an earlier work. Calculations of $\chi^{\prime \prime}$ for the longitudinal mode contain a peak near the order-parameter energy $\Delta$ in the theories of Dinter and of Schön and Ambegaokar. The characteristic frequency of this mode as derived from the Schon-Ambegaokar theury, in Llie gap regime, consists of a real part near $\Delta$ and an imaginary part equal to the spin-flip scattering rate. This calculation is in very good agreement with the data over a wide range of parameters.

A second characteristic frequency for the longitudinal mode is derived from the theorles of Dinter and of Orbacl and Entin-Wohlman. A low voltage peak in the data is in good agreement with these calculations. Dinter's theory, in particular, describes very well the $\rho-$ dependence of this mode.

Measurements of $\chi^{\prime \prime}(\omega, k)$ as a function of pair momentum $q$ were 
carried out by applying a dc transport current along the Al-Er film while recording the $I-V$ characteristics of the junction. The real and imaginary parts of the characteristic frequency of the transverse mode were reduced from the $q=0$ value. This is in qualitative agreement with a theoretical calculation of Schön and Ambegaokar. Finally, measurements of $X^{\prime \prime}(\omega, k)$ for $\mathrm{Ag} / \mathrm{Pb}$ proximity sandwich superconductors were carried out for temperatures above $\mathrm{T}_{c}$. The order-parameter diffusion rate is again reduced from the unperturbed value. The results are in excellent.agreement with a theoretical calculation of Entin-Wohlman. 
CONTENTS

LIST OF FIGURES . . . . . . . . . . . . . . . . . . . . iv

LIST OF TABLES . . . . . . . . . . . . . . . . . . v viii

I. INTRODUCTION . . . . . . . . . . . . . . . . . 1

II. THEORY . . . . . . . . . . . . . . . . . 3

A. Pair-Field Susceptibility . . . . . . . . . . 4

B. Pair-Breaking Mechanisms . . . . . . . . . . 11

C. Theory of Entin-Wohlman and Orbach . . . . . . . 16

1. Eigenfrequency Solutions . . . . . . . . 17

2. Pair-Field Susceptibility . . . . . . . 25

D. Theory of Dinter . . . . . . . . . . . 27

E. Theory of Schön and Ambegaokar . . . . . . . . . 34

1. Solutions for Zero Transport Current . . . 36

2. The Current-Carrying State . . . . . . - 41

3. Mode Softening . . . . . . . . . . . 44

F. Related Theoretical Work . . . . . . . . . 45

1. Transverse Mode Calculatione . . . . . . . 45

The Schmid Mode... . . . . . . 48

Schmid and Schön Relaxation Times . . . . 48

III. EXPERIMENTAL TECHNIQUES . . . . . . . . . . . . 50

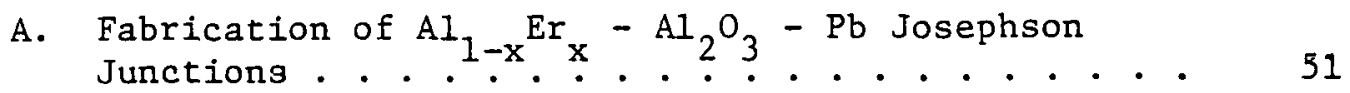

1. The Evaporation System . . . . . . . 51 
2. Preparation of the $\mathrm{Al}_{1-\mathrm{x}} \mathrm{Er}_{\mathrm{x}} \mathrm{Alloy} \cdot . \cdot . \cdot 53$

3. Fabricating the Junction . . . . . . . 54

B. Cryostat Design .. . . . . . . . . . 56

C. Data Acquisition ............... 60

IV. ANALYSIS OF THE DATA . . . . . . . . . . . . . 63

A. Normal State Tunneling Resistance... . . . . . 64

B. Quasiparticle Tunneling and Leakage Conductance . . 65

C. Pair Tunneling and Eigenfrequencies . . . . . . 68

V. RESULTS AND DISCUSSION . . . . . . . . . . . . 72

A. Data for $\mathrm{T}>\mathrm{T}_{\mathrm{C}}$. . . . . . . . . . . . 73

1. Measurements of the Pair-breaking Parameter . 73

2. Er Concentration by Auger Analysis . . . . 77

B. Data for $\mathrm{T}<\mathrm{T}_{\mathrm{C}}$. . . . . . . . . . . 82

1. Excess Current ............. 83

2. Eigenfrequencies . . . . . . . . . 99

3. The Current Corrying State . . . . . . . . . 134

L. Kelared Experlmenlal Wuik............. . 138

D. Conclusions and Suggestions for Future Work . . . . 141 ACKNOWLEDGMENTS . . . . . . . . . . . . . . . . 144 APPENDIXES

A. In $0_{\mathrm{y}}$ Barriers for Tunneling Junctions . . . . . . . 145

B. Pb/Ag Proximity Effect Tunneling Junctione . . . . . . 149 REFERENCES . . . . . . . . . . . . . . . . . 163 
LIST OF FIGURES

Figure

1. Plot of Schön-Ambegaokar theory for the high frequency longitudinal mode . . . . . . . . . . . ... 38

2. Schematic of the cryostat . . . . . . . . . . 58

3. Block diagram of the data acquisition system ...... 61

4. Contributions to the low voltage tunneling current of a Josephson current for $\mathrm{T}>\mathrm{T}_{\mathrm{c}}$. . . . . . . . . . 66

5. Typical pair current for $\mathrm{T}<\mathrm{T}_{c}$. . . . . . . . 69

6. Temperature dependence of the relaxation frequency for $\mathrm{T}>\mathrm{T}_{\mathrm{C}}$ for four samples . . . . . . . . . . . 74

7. Relaxation frequencies for $T>T_{c}$ normalized to $8\left(\mathrm{~T}-\mathrm{T}_{\mathrm{C}}\right) / \pi$. . . . . . . . C. . . . . . . . 75

8. Plot of pair-breaking energy vs. Er concentration . . . 79

9. Plot of excess current for $\Gamma=0$ and $\mathrm{Dk}^{2}=27 \mu \mathrm{V}$. . . 84

10. Plot of excess current for $\Gamma=26 \mu \mathrm{V}$ and $\mathrm{Dk}^{2}=3.7 \mu \mathrm{V} \cdot . \quad 85$

11. Plot of excess current for $\Gamma=26 \mu \mathrm{V}$ and $\mathrm{Dk}^{2}=3.7 \mu \mathrm{V} \cdot . \quad 86$

12. Rlot of excess current for $\Gamma=26 \mu \mathrm{V}$ and $\mathrm{Dk}^{2}=6.0 \mu \mathrm{V}$. . 87

13. Plot of excess current for $\Gamma=26 \mu \mathrm{V}$ and $\mathrm{Dk}^{2}=20.6 \mu \mathrm{V} \cdot{ }^{2} \cdot 88$

14. Plot of excess current for $\Gamma=26 \mu \mathrm{V}$ and $\mathrm{Dk}^{2}=33.5 \mu \mathrm{V} \cdot . \quad 89$

15. Plot of excess current for $\Gamma=26 \mu \mathrm{V}$ and $\mathrm{Dk}^{2}=20.2 \mu \mathrm{V} \cdot . \quad 90$

16. Plot of excess current for $\Gamma=26 \mu \mathrm{V}$ and $\mathrm{Dk}^{2}=30.5 \mu \mathrm{V} . .91$

17. Plot of excess current for $\Gamma=26 \mu \mathrm{V}$ and $\mathrm{Dk}^{2}=39.0 \mu \mathrm{V}$. . $92^{\circ}$

18. Plot of excess currene for $I^{\prime}=26 \mu \mathrm{V}$ and $\mathrm{Dk}^{2}=54.7 \mu \mathrm{V} \cdot . \quad 93$

19. Plot of excess current for $\Gamma=26 \mu \mathrm{V}$ and $\mathrm{Dk}^{2}=71.5 \mu \mathrm{V} \cdot . \quad 94$

20. Plot of excess current for $\Gamma=26 \mu \mathrm{V}$ and $\mathrm{Dk}^{2}=120.0 \mu \mathrm{V} .95$ 
Figure

21. Plot of excess current for $\Gamma=39 \mu \mathrm{V}$ and $\mathrm{Dk}^{2}=14.7 \mu \mathrm{V} . \quad 97$

22. Plot of excess current for $\Gamma=41 \mu \mathrm{V}$ and $\mathrm{Dk}^{2}=21.0 \mu \mathrm{V} . .98$

23. Gap mode eigenfrequencies for $\Gamma=0, \mathrm{Dk}^{2}=27 \mu \mathrm{V}$

with $\mathrm{SA}$ and $\mathrm{OEW}$ theories............. 100

24. Gap mode eigenfrequencies for $\Gamma=26 \mu \mathrm{V}, \mathrm{Dk}^{2}=20.2 \mu \mathrm{V}$

with SA theory................. . 101

25.- Gap mode eigenfrequencies for $\Gamma=26 \mu \mathrm{V}, \mathrm{Dk}^{2}=39 \mu \mathrm{V}$

with SA theory. . . . . . . . . . . . . 102

26. Gap mode eigenfrequencies for $\Gamma=26 \mu \mathrm{V}, \mathrm{Dk}^{2}=54.7 \mu \mathrm{V}$

with SA theory................. 103

27. Gap mode eigenfrequencies for $\Gamma=26 \mu \mathrm{V}, \mathrm{Dk}^{2}=71.5 \mu \mathrm{V}$
with SA theory.................. 104

28. Gap mode eigenfrequencies for $\Gamma=39 \mu \mathrm{V}, \mathrm{Dk}^{2}=14.7 \mu \mathrm{V}$ with SA theory.................. 105

29. Gap mode eigenfrequencies for $\Gamma=39 \mu \mathrm{V}, \mathrm{Dk}^{2}=14.7 \mu \mathrm{V}$ with OEW theory ................. 106

30. L-mode eigenfrequencies for $\Gamma=26 \mu \mathrm{V}, \mathrm{Dk}^{2}=20.2$, $39.0,54.7$ and $71.5 \mu \mathrm{V}$ with OEW theory . . . . . . 107

31. L-mode eigenfrequencies for $\Gamma=26 \mu \mathrm{V}, \mathrm{Dk}^{2}=20.2$
$39.0,34.1$ and $71.5 \mu \mathrm{V}$ w1th DLnLeL"'s theory....... 108

32. L-mode and $T$-mode eigenfrequencles fur $\Gamma-0$, $\mathrm{Dk}^{2}=27 \mu \mathrm{V}$ with $\mathrm{OEW}$ theory ............ 110

33. L-mode and $\mathrm{T}$-mode eigenfrequencies for $\Gamma=0$, $\mathrm{Dk}^{2}$. $=27 \mu \mathrm{V}$ with Dinter's theory . . . . . . . . . . 111

34. $\mathrm{L}$-mode and $\mathrm{T}$-mode eigenfreqieucnes for $\Gamma=39 \mu \mathrm{V}$, $\mathrm{Dk}^{2}=14.7 \mu \mathrm{V}$ with $\mathrm{OEW}$ theory ............. . 112

35. L-mode and $T$-mode eigenfrequencies for $\Gamma=39 \mu \mathrm{V}$, $\mathrm{Dk}^{2}=14.5 \mu \mathrm{V}$ with Dinter's theory .. . . . . . . . 113

36. L-mode and $T$-mode eigenfrequencies for $\Gamma=41 \mu \mathrm{V}$, $\mathrm{Dk}^{2}=21 \mu \mathrm{V}$ with OEW theory ... . . . . . . . . 114

37. L-mode and $T$-mode eigenfrequencies for $\Gamma=41 \mu \mathrm{V}$, $\mathrm{Dk}^{2}=21 \mu \mathrm{V}$ with Dinter's theory . . . . . . . . 115 
Figure

38. T-mode eigenfrequencies for $\Gamma=41 \mu \mathrm{V}, \mathrm{Dk}^{2}=21 \mu \mathrm{V}$

with SA theory... . . . . . . . . . . . .

39. T-mode eigenfrequencies for $\Gamma=0, \mathrm{Dk}^{2}=27 \mu \mathrm{V}$

with SA theory .. . . . . . . . . . . . . . .

40. T-mode eigenfrequencies for $\Gamma=39 \mu \mathrm{V}, \mathrm{Dk}^{2}=14.7 \mu \mathrm{V}$

with SA theory. . . . . . . . . . . . . . . . .

41. T-mode eigenfrequencies for $\Gamma=26 \mu \mathrm{V}, \mathrm{Dk}^{2}=20.2 \mu \mathrm{V}$ with OEW theory... . . . . . . . . . . . . . .

42. T-mode eigenfrequencies for $\Gamma=26 \mu \mathrm{V}, \mathrm{Dk}^{2}=20.2 \mu \mathrm{V}$ with Dinter's theory . . . . . . . . . . . . . . .

43. T-mode eigenfrequencies for $\Gamma=26 \mu \mathrm{V}, \mathrm{Dk}^{2}=20.2 \mu \mathrm{V}$ with SA theory . . . . . . . . . . . . . . . . .

44. T-mode eigenfrequencies for $\Gamma=26 \mu \mathrm{V}, \mathrm{Dk}^{2}=39.0 \mu \mathrm{V}$ with OEW theory . . . . . . . . . . . . . . . .

45. T-mode eigenfrequencies for $\Gamma=26 \mu \mathrm{V}, \mathrm{Dk}^{2}=39.0 \mu \mathrm{V}$ with Dinter's theory . . . . . . . . . . . . .

46. T-mode eigenfrequencies for $\Gamma=26 \mu \mathrm{V}, \mathrm{Dk}^{2}=39.0 \mu \mathrm{V}$ with SA theory....................

47. T-mode eigenfrequencies for $\Gamma=26 \mu \mathrm{V}, \mathrm{Dk}^{2}=54.7 \mu \mathrm{V}$ with OEW theory . . . . . . . . . . . . . . .

48. T-mode eigenfrequencies for $\Gamma=26 \mu \mathrm{V}, \mathrm{Dk}^{2}=54.7 \mu \mathrm{V}$ with Dinter's theory . . . . . . . . . . . . .

49. T-mode eigenfrequencies for $\Gamma=26 \mu \mathrm{V}, \mathrm{Dk}^{2}=54.7 \mu \mathrm{V}$ with SA theory. . . . . . . . . . . . . . . .

50. T-mode eigenfrequencies for $\Gamma=26 \mu \mathrm{V}, \mathrm{Dk}^{2}=71.5 \mu \mathrm{V}$ with OEW theory

51. T-mode eigenfrequencies for $\Gamma=26 \mu \mathrm{V}, \mathrm{Dk}^{2}=71.5 \mu \mathrm{V}$ with Dinter's theory . . . . . . . . . . . . .

52. T-mode eigenfrequencies for $\Gamma=26 \mu \mathrm{V}, \mathrm{Dk}^{2}=71.5 \mu \mathrm{V}$ with SA theory... . . . . . . . . . . . . .

53. SA theory with finite pair momentum and eigenfrequencies for $\Gamma=0, \mathrm{Dk}^{2}=27 \mu \mathrm{V}$ and $\varepsilon=0.005$ 
Figure

54. SA theory with finite pair momentum and eigenfrequencies for $\Gamma=26 \mu \mathrm{V}, \mathrm{Dk}^{2}=0.8 \mu \mathrm{V}$ and $\varepsilon=0.007 . \cdot 136$

55. P1ot of $\mathrm{T}_{\mathrm{C}}$ vs $\mathrm{Pb}$ film thickness for a number of $\mathrm{Ag} / \mathrm{Pb}$ proximity superconductors . . . . . . . . 155

56. Representative plot of $\mathrm{I}_{\mathrm{ex}} \mathrm{vs} \mathrm{V}$ for an $\mathrm{Ag} / \mathrm{Pb}-\mathrm{Pb}$ junction, with quasi-Lorentzian superimposed . . . . . . 157

57. P1ot of $\mathrm{V}_{\mathrm{p}}$ vs $\mathrm{T}-\mathrm{T}_{\mathrm{c}}$ for four $\mathrm{Ag} / \mathrm{Pb}-\mathrm{Pb}$ junctions . . 159

58. Plot of $\omega_{R}$ normalized to $8\left(T-T_{C}\right) / \pi$ plotted as a function of $\mathrm{T}-\mathrm{T}$ for three $\mathrm{Ag} / \mathrm{Pb}-\mathrm{Pb}$ junctions. 160 
Table

I. Pair-breaking energy determined from relaxation frequencies and from Er concentrations . . . . . . . .

II. Physical parameters of $\mathrm{Al}_{1-\mathrm{x}} \mathrm{Er}_{\mathrm{x}}$ electrodes and of junction barriers ... . . . . . . . . . . . .

III. Expressions for the real and imaginary parts of the transverse-mode eigenfrequency in the gap regime given by $i \omega=\omega_{D}+i\left(c^{2} k^{2}-\omega_{D}^{2}\right)^{1 / 2}$ for each of

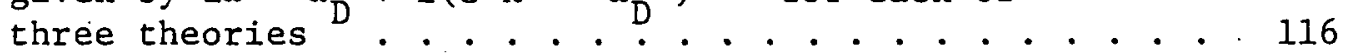

IV. Physical parameters of several $\mathrm{Ag} / \mathrm{Pb}-\mathrm{Pb}$ tunneling junctions . . . . . . . . . . . . . . . 158

V. Sumnary of the parameters relevant to the Ginzburg-Landau diffusion rate . . . . . . . . . . 


\section{INTRODUCTION}

The pair-field susceptibility of the superconducting order parameter and its relation to a measurable tunneling current in a Josephson junction is by now established as perhaps the most sensitive probe of order parameter dynamics. Of particular interest are the related dispersion relations for the order parameter fluctuations from which the characteristic frequencies can be calculated. Of more recent interest is the effect of a pair-breaking mechanism such as spin-flip scattering on the dynamics of the order parameter. Experimental measurements of the imaginary part of the pairfield susceptibility for Al-Er alloys exhibiting moderate spin-flip scattering are presented in this dissertation. Characteristic frequencies are extracted from these measurements and compared in detail to current theories. Alloys with Er concentrations as high as $1 \%$ are examined at temperatures within $20 \%$ of $\mathrm{T}_{c} \cdot$ Although the alloy $\mathrm{Al}_{0.99^{\mathrm{Er}} 0.01}$ is gapless for a wide range of temperatures below $\mathrm{T}_{c}$, it is still far from the extreme gapless limit. Attempts to reach this limit with $\mathrm{Pb} / \mathrm{Ag}$ proximity sandwich superconductors met with 1 imited success and is discussed in the appendix.

It is the purpose of this dissertation to present the first measurements of the imaginary part of the pair-field susceptibility influenced by spin-flip scattering. Furthermore, it is the purpose 
of this dissertation to test existing theories which calculate the pair-field susceptibility and to develop these theories where possible to make the most meaningful comparison to the data.

Some.knowledge of the subject is assumed throughout the discussion. Brief reviews of the theoretical results for the pairfield susceptibility and for the Abrikosov and Gor'kov theory are given along with adequate references for the reader unfamiliar with the subject. This is followed by a detailed description of the experimental techniques, including junction fabrication, cryostat design and data acquisition. Reduction of the data into a form which can be compared to theory is then discussed. Finally detailed comparison of the data to three theories is given. Experimental work performed by the author on artificial In $\mathrm{O}_{\mathrm{y}}$ tunneling barriers and on $\mathrm{Pb} / \mathrm{Ag}$ proximity effect junctions is of interest to workers in the field and is discussed in Appendix A and B respectively. 
II. THEORY

To provide a background for the theory specific to this work, a brief summary will be given of the theories of Ferrell ${ }^{1}$ and Scalapino, ${ }^{2}$ in which the imaginary part of the pair-field susceptibility is shown to be proportional to a measurable excess current in the tunneling characteristics of a Josephson junction. Further background will be provided in sections in which various pairbreaking mechanisms are discussed.

The balance of this chapter will be devoted to the examination of several theories which calculate the characteristic frequencies of order-parameter fluctuation and the pair-field susceptibility. Where possible, detailed expressions will be given for the eigenfrequency equations and/or the pair-field susceptibility. Emphasis will be placed on the effects of a pair-breaking mechanism.

The fundamental constants $k_{B}$, $x, c$ and $e$ will be used explicitly during the introduction of the parameters. Except whore deemed necessary for the sake of clarity they will otherwise be set equal to unity which is in accordance with standard practice. 


\section{A. PAIR-FIELD SUSCEPTIBILITY}

An excellent review of the derivation of the pair-field susceptibility as it pertains to this work is given by Carlson. 3,4 A more recent account is given in the review article by Giaquinta and Mancini. ${ }^{5}$ Only the important results and definition of the variables will be presented here.

Any reference to tunneling will refer to tunneling between a reference superconductor (which is $\mathrm{Pb}$ for all experiments reported here) and the superconductor of interest (such as Al-Er alloys). Primed variables will refer to the reference superconductor. The superconducting transition temperatures are denoted $\mathrm{T}_{c}{ }^{\prime}$ and $\mathrm{T}_{\mathrm{c}}$. Characteristic lengths of interest are the penetration depth $\lambda$, the coherence length $\xi$ and the electrode thickness d of each superconductor. The area of the tunneling junction is $A$ and the normal state tunneling resistance is $\mathrm{R}_{\mathrm{N}}$. The experiments take place at temperatures $\mathrm{T} \simeq \mathrm{T}_{\mathrm{C}} \ll \mathrm{T}_{\mathrm{C}}{ }^{\prime}$.

The usual single particle tunneling (often referred to as quasiparticle tunneling) between 3 superconductor and a normal metal or between two superconductors is well understood and parametrized. 6 By excess current $I_{\text {ex }}$ we shall mean currents associated with the tunneling of electron pairs (Cooper pairs) at finite voltage which are in excess of the single-particle current. This excess dc current is related to but not the same as the ac Josephson current. For the ac Josephson effect the two electrodes separated in space by an insulating barrier can be represented by two energy states that are separated in energy by $2 \mathrm{eV}$, where $\mathrm{V}$ is the potential-difference 
between them. A consequence of quantum mechanics is that Cooper pairs will oscillate between the two states at a frequency of $\omega=2 \mathrm{eV} / \mathrm{K}^{\circ}$. Since the states are spatially separated by an insulating layer, the pairs will also tunnel through the barrier at the same rate. Cooper pairs on the high energy side of the barrier will decay into normal electrons or thermally excited quasiparticles at a higher rate than on the low energy s1de. These normal electrons cannot tunnel back to the low energy side. which rejults in a net dc tunneling of superconducting pairs.

A complete formal description of this mechanism involves spatial and temporal fluctuations of the two components of the superconducting order parameter. It is usual to choose the average phase of the order parameter to be zero; that is, the equilibrium order parameter ls Laker tu be red. Then flucluations of the real part are synonymous with fluctuations of the modulus of the order parameter. In like manner phase fluctuations are proportional to fluctuations. in the imaginary part of the order parameter.

The picture of dc pair tunneling given above is related to modulus fluctuations, and is referred to as the longitudinal mode. It can be observed both above $T_{c}$ where the average value of the order parameter is zero and below $\mathrm{T}_{c}$ where the order parameter is finite. Phase fluctuations on the other hand are meaningless above $T_{c}$ since the average value of the order parameter is zero. Below $T_{C}$ fluctuations in the phase are coupled to fluctuations of the quasiparticle distribution function via what's known as the anomalous term. ${ }^{7}$ The result is a damped wave equation for the order parameter 
fluctuations instead of the diffusion equation which describes fluctuations above $\mathrm{T}_{c}$. This type of order parameter fluctuation is referred to as the transverse mode.

The excess current due to pair tunneling appears at low voltages $\left(\mathrm{V} \ll \mathrm{k}_{\mathrm{B}} \mathrm{T} / \mathrm{e}\right)$ at temperatures near $\mathrm{T}_{\mathrm{C}}$. It is directly proportional to the imaginary part of the pair-field susceptibility and is given by

$$
I_{e x}(V, H)=\left(4 e|\bar{C}|^{2} A / d K\right) \chi^{\prime \prime}(\omega, k),
$$

where $H$ is the magnetic field applied in the plane of the junction, -e is the charge on an electron, $h$ is Planck's constant divided by $2 \pi, \bar{C}$ is the coupling constant given by

$$
\bar{C}=\left(\ln 4 T_{C}{ }^{\prime} / T_{C}\right) / R_{N} A,
$$

and $\chi^{\prime \prime}$ is the imaginary part of the pair-field susceptibility. The Josephson frequency and wave number are given by

$$
\omega=2 \mathrm{eV} / \text { Һ }
$$

and

$$
\cdot k=\left(2 e / K_{c}\right) H\left(\lambda^{\prime}+d / 2\right) \text {. }
$$

For temperatures greater than $\mathrm{T}_{c}$ it was predicted by Scalapino that the excess current would take the form

$$
I_{e x}=\left(2 I_{p} V / V_{p}\right) /\left(1+\left(V / V_{p}\right)^{2}\right)
$$

where 


$$
v_{p}=(x / 2 e) \Gamma_{0}\left(1+k^{2} \xi^{2}(T)\right)
$$

and

$$
I_{p}^{-1}=\left(d N(0)\left(T-T_{c}\right) / 2 e A|\bar{C}|^{2} T_{c}\right)\left(1+k^{2} \xi^{2}(T)\right)
$$

Here $\Gamma_{0}$ is the pair relaxation frequency in zero field given by

$$
\Gamma_{0}=8 k_{B}\left(T-T_{C}\right) / \pi \hbar \text {. }
$$

$N(0)$ is the electronic density of states measured relative to the Fermi level. The temperature-dependent coherence length $\xi(T)$ is given in terms of the BCS coherence length $\xi_{0}$, the electronic mean free path $\ell$, and the reduced temperature $\varepsilon$.

$$
\begin{array}{ll}
\xi(T)=.85\left(\xi_{0} \ell / \varepsilon\right)^{1 / 2} & \text { for } \ell \ll \xi_{0}, \\
\xi(T)=.74 \xi_{0} / \varepsilon^{1 / 2} & \text { for } \ell \gg \xi_{0},
\end{array}
$$

and $\varepsilon$ is defined by

$$
\varepsilon=\left(T-T_{c}\right) / T_{c}
$$

Combining the dirty limit (Eq. (9a)) with the definition for the diffusion constant $D=V_{F} l / 3$ and the BCS coherence length $\xi_{0}=0.18 \mathrm{KV} \mathrm{F}_{\mathrm{B}} / \mathrm{k}_{\mathrm{B}} \mathrm{T}_{\mathrm{c}}$, one obtains the dirty-limit diffusion constant

$$
D=\left(8 \mathrm{k}_{\mathrm{B}} \mathrm{T}_{\mathrm{C}} / \pi \mathrm{K}\right) \xi^{2}(0)
$$

Then the dirty-limit form of Eq. (6) can be written

$$
v_{p}=(\not / 2 e)\left(\Gamma_{0}+D k^{2}\right) .
$$


Several experimental tests ${ }^{8-11}$ were quick to follow and offered qualitative support for Scalapino's theory. In the work of Anderson and Goldman ${ }^{8,9}$ measurements of the current-voltage characteristics of $\mathrm{Pb}-\mathrm{Sn}_{\mathrm{x}} \mathrm{O}_{\mathrm{y}}-\mathrm{Sn}$ were made and found to be in qualitative agreement with theory. By refining the data acquisition techniques Anderson, Carlson and Goldman ${ }^{12}$ and Carlson and Goldman ${ }^{3,4}$ were able to quantitatively verify the theory. In order to reduce the background current due to single-particle tunneling $\mathrm{Al}-\mathrm{Al} \mathrm{x}_{\mathrm{y}} \mathrm{O}-\mathrm{Pb}$ junctions were used instead of $\mathrm{Sn}-\mathrm{Sn} \mathrm{x}_{\mathrm{y}}-\mathrm{Pb}$ junctions. From the graph of $\mathrm{V}_{\mathrm{p}}$ as a function of temperature it was found that the slope was consistent with the theoretical value $4 \mathrm{k}_{B} / \pi$ e predicted in Eq. (12). Also, a plot of $\mathrm{V}_{\mathrm{p}}$ as a function of $\mathrm{H}^{2}$ at constant $\mathrm{T}-\mathrm{T}_{\mathrm{c}}(\mathrm{H})$ yielded a straight line as predicted. Having verified the theory one can turn Eq. (12) around and use the plot of $V_{p}$ versus $H^{2}$ to determine $\mathrm{Dk}^{2}$ with far more accuracy than is possible from a calculation based on the physical parameters contained in Eqs. (4) and (11). Carlson and Goldman $3,4,13,14$ extended the experiment to temperatures below $I_{C}$ and found that sralapinn's thenry broke down. In place of the simple quasi-Lorentzian observed above $T_{c}$, they found two sharp peaks and a low-frequency "shoulder" in the excess currentvoltage characteristics.

of particular interest was the mid-frequency peak (now known as the Carlson-Goldman mode). The real part of its characteristic frequency exceeded the imaginary part indicating the presence of a propagating mode. Several theories ${ }^{15-20}$ were advanced to explain this structure. Good agreement between theory ${ }^{15,16}$ and experiment 
was obtained by explaining the mode as a transverse order parameter mode, i.e. due to fluctuations in the phase of the order parameter. The "anomalous" term in thè Green's function allows coupling of the phase fluctuations to fluctuations of the quasiparticle distribution function via the chemical potential. The coupling can occur. only for frequencies greater than inelastic scattering rates due to such processes as inelastic electron-phonon scattering and spinflip scattering. Working in the limit of negligible inelastic scattering Schmid and Schön ${ }^{16}$. were able to derive the following microscopic equations for the superconducting and normal parts of the electron density. $\ddot{\vec{u}}_{s}=c^{2} \nabla\left(\nabla \cdot \vec{u}_{s}\right)+(e / m) \vec{E} ; \dot{\vec{u}}_{n}=(e r / m) \vec{E}$. Here $\vec{E}$ is the local electric field and $\tau$ is the collision frequency. The first equation describes the local acceleration of the superconducting part and the second equation is Ohm's law for the normal part. The second contribution to $\ddot{\vec{u}}_{s}$ is the acceleration of a free charge in an electric field. The first contribution is the gradient of the scalar potential $\nabla \cdot \overrightarrow{\mathrm{u}}_{\mathrm{s}}$. Here $\nabla \cdot \overrightarrow{\mathrm{u}}_{\mathrm{s}}$ can be identifled w1th deviations of the chemical potential of the superconducting electrons from equilibrium. Then $\nabla\left(\nabla \cdot \vec{u}_{s}\right)$ represents the force restoring the superconducting part to its equilibrium position. The equations given above along with Maxweli's equation $\nabla \cdot \vec{E}=-4 \pi e \nabla \cdot\left(n_{S} \vec{u}_{S}+n_{n} \vec{u}_{n}\right)$ relating the electric field to the charge densities $n_{s}$ and $n_{n}$ result in a wave-like solution of the form $\exp (i \vec{k} \cdot \vec{r}-i \omega t)$ where $\omega=i \pi \Delta^{2} / 4 \hbar k_{B} T$ $+\left[(2 \Delta \mathrm{D} / \mathrm{K}) \mathrm{k}-\left(\pi \Delta^{2} / 4 \pi \mathrm{k}_{\mathrm{B}} \mathrm{T}\right)^{2}\right]^{1 / 2}$ and the wave velocity is $\mathrm{c}=$ $\sqrt{2 \Delta \mathrm{D} / \mathrm{h}}$.

For large inelastic scattering one obtains a diffusion equation 
similar to that obtained for $\mathrm{T}>\mathrm{T}_{\mathrm{c}}$ instead of a wave equation. The primary motivation for this work is to investigate in a controlled manner this change in the transverse mode.

The low frequency shoulder was determined to be due to fluctuations in the magnitude of the order parameter, referred to as longitudinal fluctuations.

The high frequency peak known as the secondary peak or gap peak occurs at $V \simeq \Delta$. This peak has also been observed by Burratato et al. ${ }^{23}$ in A1-Sn junctions. It was believed that this peak was not contained in the pair-field susceptibility but was due to single particle tunneling. The primary motivation for this belief was the relative insensitivity of the peak amplitude and peak voltage to an applied magnetic field.

Simanek and Hayward ${ }^{21}$ were the first to propose a theory to explain this feature. In their theory an ac voltage induced in the fluctuating superconductor by the ac Josephson effect induces oscillations in $\Delta(k, t)$ which contribute to the dc current. The threshold for this process is $V=\Delta$. Rounding of the current $j$ ump is provided by pair-breaking effects of the magnetic field and finite conduction electron mean free path. The exact nature of the gap peak is determined by the relative phase of the ac Josephson current and the induced voltage.

At about the same time Baramidze and Cheishvili ${ }^{22}$ calculated the effects of fluctuational pairing on electron tunneling. Their Green's function calculation shows a sharp rise in current at $V=\Delta . \quad$ The solution contains the hypergeometric function 
$F(3 / 2,3 / 2,5 / 2 ;(1-i \gamma) / 2)$ where $\gamma=\pi(V-\Delta) / 4\left(T-T_{c}\right)$. It may be deduced from their solution in finite field that the width of the gap peak is eHD. In zero field the width is $4\left(T-T_{c}\right) / \pi$.

Two recent theories 24,25 indicate there is a gap peak in the longitudinal mode of the pair-field susceptibility. These theories will be discussed in detail in sections $D$ and $E$ of this chapter. Other theoretical calculations of characteristic frequencies w111 be summalzed L! secliún $F$.

\section{B. PAIR-BREAKING MECHANISMS}

A detailed discussion of this subject may be found in the works of Maki $^{26}$ and Fulde ${ }^{27}$ and as it pertains to magnetic impurities by Maple. ${ }^{28}$ Superconductivity is understood as the correlation of electron. pairs of opposite spin and momentum. Under time reversal the electron states are simply interchanged, and thus the correlation is unaffected. If a perturbation breaks the time reversal symnetry, it is not surprising that the superconducting properties are severely affected. Magnetic impurities, for example, acting as spin-flip scattering sites, break time-reversed states and thus reduce the superconducting electron density. Anderson ${ }^{29}$ proved that nonmagnetic impurities do not affect the thermodynamic properties of a superconductor. A more general form of his theorem is: ${ }^{26}$ "If a static external perturbation does not break the time-reversal symmetry and does not cause a longrange spatial variation of the order parameter the thermodynamic properties of the superconductor remain unchanged in the presence of 
the perturbation." This conclusion was reached independently by Abrikosov and Gor'kov. 30

There are many time-reversal symmetry-breaking mechanisms that act to destroy superconductivity. Examples of such mechanisms 26,27 are magnetic fields, magnetic impurities, supercurrents, and spatial variation of the superconducting order parameter. For any such mechanism there is an associated depairing energy $九 \Gamma$. It is useful to define the pair-breaking parameter $\rho$.

$$
\rho=\mathbf{n} \Gamma / 2 \pi k_{B} T \text {. }
$$

In many cases if more than one pair-breaking mechanism is present, the effective $\rho$ is the sum of each contributing $\rho .{ }^{31}$ The presence of a pair-breaking mechanism has several quantitative effects on the supercondoctor. The transition temperature is reduced to $T_{c}\left(\rho_{c}\right)$ given by ${ }^{32}$

$$
\ln \left(\mathrm{T}_{c o} / \mathrm{T}_{c}\left(\rho_{c}\right)\right)=\psi\left(1 / 2+\rho_{c}\right)-\psi(1 / 2)
$$

where $\mathrm{T}_{c o}$ is the transition temperature in the absence of the pairbreaking mechanism. $\psi(\mathrm{X})$ is the digamma.function. For the range of temperatures below $T_{c}$ where $h \Gamma>\Delta(T)$ the superconductor is gapless. Here $\Delta(T)$ is the order parameter of the superconductor. O. Entin-Wohlman and R. Orbach ${ }^{33}$ calculated the effect of magnetic impurities on the pair-field susceptibility for temperatures greater than $T_{c} \cdot$ It can be deduced from their work that $x^{-1}(\omega, k)=N(0)\left[\ln \left(T / T_{c}\right)+\psi\left(1 / 2+\rho+\left(n k^{2}-i \omega\right) / 4 \pi T\right)-\psi\left(1 / 2+\rho_{c}\right)\right]$, 
where $\rho_{c}=\rho\left(T_{c}\right)$. The corresponding $I-V$ characteristic is again a quasi-Lorentzian line shape with a relaxation frequency reduced from the $\rho=0$ case. For $T-T_{c} \ll T_{c}$ the peak voltage is

$$
v_{p}=(\hbar / 2 e)\left(\Gamma_{o} f(\rho)+D k^{2}\right)
$$

where

$$
f(\rho)=\left(1-\rho \psi^{\prime}(1 / 2+\rho)\right) \psi^{\prime}(1 / 2) / \psi^{\prime}(1 / 2+\rho)
$$

Thus the graph of $V_{p}(T)$ is still linear with a slope reduced from the $\rho=0$ case by a factor $f(\rho)$. By carefully measuring $V_{p}$ for $T>T_{C}$ one can determine the value of the pair-breaking parameter $\rho$. Naively one might expect the pair relaxation frequency to increase rather than decrease when a pair-breaking process is present. However, one should consider that the presence of a pair-breaking mechanism weakens superconductivity as is reflected in a longer coherence length. 34 The coherence length is increased by a factor $\mathrm{f}^{-1 / 2}(\rho)$ where $f(\rho)$ is given by Eq. (17). The pair relaxation trequency is actually the reciprocal of the diffusion time for electrons to travel. a distance of the order of the coherence length. Thus an increased coherence length resulting from a weakening of superconductivity would be expected to result in a decrease in the relaxation frequency. 35

The most obvious effects of pair-breaking on the pair-field susceptibility, however, should be those related to the transverse mode. As stated earlier, propagation of the mode requires $\omega \tau \gg 1$ where $\tau^{-1}$ is the inelastic scattering rate (superconducting-normal conversion (1) $)$. The primary motivation for this work was to investigate the dependence of the propagating mode on the scattering rate $\tau^{-1}$. In 
general ${ }^{25} \tau^{-1}=1 / 2 \tau_{E}+1 / \tau_{S}+\ldots$ where $\tau_{E}$ is the inelastic electronphonon scattering time, $\tau_{S}$ is the spin-flip scattering time and all other pair-breaking mechanisms contribute to $\tau^{-1}$. By increasing $\tau^{-1}$ one should cause the transverse mode. to cross from the high-frequency propagating regime to a low-frequency diffusive regime.

In our experiments several pair-breaking mechanisms may be present simultaneously. When this is the case, the pair-breaking parameters are additive and are represented by one overall pair-breaking parameter. For the experiments reported here the pair-breaking energy due to the magnetic field is about $x \mathrm{Dk}^{2} / 20$. This is negligible compared to the spin-flip scattering rate for all but the lowest concentrations of magnetic impurities. The pair-breaking energy due to supercurrents is $\leq \hbar D q_{c}{ }^{2}$ where $q_{C}=1 / \sqrt{3 \xi(T)}$ is the critical pair momentum. Again $\mathrm{hDq}_{\mathrm{c}}{ }^{2}$ is small and is not effective as a pairbreaking parameter at temperatures near $\mathrm{T}_{c}$. The presence of pair momentum does have other significant effects on the pair-field susceptibility which will be examined in detail in section E. Pair-breaking due to magnetic impurities is significant and does not interfere with the excess current in any way other than as a pair-breaking mechanism. For these reasons this mechanism is believed to be the primary source of depairing in these experiments. The strength of the pair-breaking can be controlled since it is proportional to the concentration of magnetic impurities in the superconductor. Concentrations of the order of a few tenths of a percent are sufficient to exhibit strong palr-breaking effects but not so strong as to destroy superconductivity completely. The Al-Er system studied here is 
especially useful since $\mathrm{Er}$ and $\mathrm{Al}$ have comparable vapor pressures making joint evaporation possible and Er exhibits a localized moment in Al for low enough concentrations. 36

In the first Born approximation the pair-breaking energy $\Gamma$ is linearly proportional to the magnetic impurity concentration $C{ }^{32}$

$$
\Gamma=(\pi / 2) C J_{S f}{ }^{2} N\left(E_{F}\right)\left(g_{J}-i\right)^{2} J(J+1)
$$

Here $J_{S f}$ is the exchange Lilleglal, $N\left(E_{F}\right)$ is the single spin denaiey of states at the Fermi level, $g_{J}$ is the Lande $g$-factor and $J$ is the total angular momentum. From Abrikosov-Gor'kov theory

$$
\ln \left(T_{c o} / T_{c}\right)=\psi\left(1 / 2+\rho_{c}\right) \div \psi(1 / 2) .
$$

Here $T_{c o}$ is the unperturbed transition temperature and $T_{c}=T_{c}\left(\rho_{c}\right)$. For $\varepsilon \lll 1$ and $\rho_{c} \lll 1$ Eq. (19) reducee to

$$
\left(T_{c o}-T_{c}\right) \simeq \psi^{\prime}(1 / 2) \rho_{c \cdot} T_{c}=\pi \Gamma / 4
$$

Combining Eqs. (18) and (20) one gets for the depression of, $T_{c}$

$$
\left(T_{c o}-T_{c}\right)=\left(\pi^{2} / 8\right) C_{. I}{ }_{s f}^{2} N\left(E_{F}\right)\left(g_{J}-1\right)^{2} J(J+1)
$$

Unfortunately, $T_{c o}$ for $A l$ depends strongly on the normal electron meanfree-path. Since magnetic impurities can act as normal electron scattering sites as well as spin-flip scattering sites, $T_{\text {co }}$ itself is a function of $\mathrm{C}$.

Let us now examine in detail three theories which calculate the effect of pair-breaking on the characteristic frequencies of the collective modes below $\mathrm{T}_{\mathrm{c}}$. 
C. THEORY OF ENTIN-WOHLMAN AND ORBACH

This theory ${ }^{37}$ is based on the coupling of a time-dependent Ginzburg-Landau equation for the order parameter to a Boltzmann equation for the quasi-particle distribution function. The calculation is similar to that of Schmid and Schön ${ }^{15,16}$ but includes the effects of magnetic impurities as well as inelastic phonon scattering. They confirm the separation of order parameter fluctuations into longitudinal (real) and transverse (imaginary) components and the decoupling of the fluctuations of the real and imaginary parts.

General expressions containing integrals that can be solved in various limits are derived for the eigenfrequencies and the pair-field susceptibility. Analytical expressions are given for the longitudinal and transverse susceptibilities with and without magnetic impurities in the gap and gapless regimes and for gapless superconductors.

In the following general forms for the transverse mode and longitudinal mode dispersion relations will be presented. The solutions of the integrals and the resulting dispersion relationo will then be given for each special case. The general expressions for the pair-field susceptibility are then given, followed by each of the special case forms. 
1. Eigenfrequency Solutions

The transverse mode dispersion equation is

$$
\omega^{2}-\Delta \mathrm{TDk}^{2} \mathrm{a} / \mathrm{I}_{4}+2 \Delta^{2} \mathrm{Ta} / \mathrm{I}_{2}=0
$$

where

$$
a=\psi^{\prime}(1 / 2+0) / 2 \pi T-\omega I_{5} / \Delta T
$$

and $I_{2}, I_{4}$ and $I_{5}$ are integrals that depend on $\omega, D k^{2}, T, \Gamma$ and $\Delta$. These integrals can be solved for special cases.

To solve the dispersion relation in the gap regime $(\Delta \gg \Gamma) M_{1}$ is defined

$$
M_{1}(x)=w\left(1+x^{2}\right)^{2}+i D k^{2}\left(1-x^{4}\right)+8 i \Gamma x^{2}
$$

Then the integrals are

$$
I_{2} \equiv=i T\left(1-\Delta x_{2} / T\right) /\left(\omega_{1}+i n k^{2}\right)
$$

where

$$
\begin{aligned}
x_{2} & =\int_{0}^{1} \operatorname{dx}\left(2 i \Gamma\left(1-\dot{x}^{2}\right)-i / 2 D^{2}\left(1-x^{4}\right) / M_{1}(x)\right) \\
I_{4} & =\underset{0}{1 / 2 \int d x}\left(1+x^{2}\right)(\omega+2 i \Gamma) / M_{1}(x)
\end{aligned}
$$

and.

$$
\left.I_{5}=\underset{0}{i / 2\left[\int d x\right.}\left(2 i \Gamma\left(I-\dot{x}^{2}\right)-i D k^{2}\left(1+x^{2}\right)\right) / M_{1}(x)+0(\ln \Delta / \omega)\right] .
$$

To leading order it is found that

$$
\begin{aligned}
& I_{2}=T /\left(i \omega-\mathrm{Dk}^{2}\right), \\
& I_{4}=\pi / 8
\end{aligned}
$$

and 


$$
a^{\prime}=\dot{\psi}^{\prime}(1 / 2+\rho) / 2 \pi \mathrm{T} \text {. }
$$

Inserting Eq. (29) - Eq. (31) into Eq. (22) the dispersion relation becomes

$$
\omega^{2}-4 \gamma \mathrm{TDk}^{2} / \pi \Delta+i \omega \gamma=0,
$$

where

$$
\gamma=\Delta^{2} \psi^{\prime}(1 / 2+0) / \pi \mathrm{T}
$$

The solution for the transverse mode eigenfrequency in the gap regime is

$$
\omega=-i \gamma / 2 \pm\left[-\gamma^{2} / 4+4 \gamma \mathrm{TDk}^{2} / \pi \Delta\right]^{1 / 2} .
$$

This is a propagating mode with group velocity

$$
\mathrm{V}_{\text {mode }}=\left(2 \Delta \mathrm{D} \psi^{\prime}(1 / 2+\rho) / \psi^{\prime}(1 / 2)\right)^{1 / 2}
$$

and damping

$$
\operatorname{In}(\omega)=\gamma / 2 \text {. }
$$

In the gapless regime the integrals become

$$
\begin{aligned}
& I_{2}=\frac{-i T}{\omega+i D k^{2}}\left[1-\frac{i 2 \Delta^{2} / \Gamma \psi^{\prime}(1 / 2+\rho)}{\left(\omega+i D k^{2}\right)(2 \pi T)^{2} \rho_{1}}\right], \\
& I_{4}=i \Delta \psi^{\prime}\left(1 / 2+\rho_{1}\right) \rho / 2 \pi\left(\omega+i D k^{2}\right) \rho_{1},
\end{aligned}
$$

and

$$
I_{5}=i I_{4} \text {, }
$$


where

$$
\rho_{1}=\rho\left(1+2 i \Delta^{2} / \Gamma\left(\omega+i D k^{2}\right)\right)^{1 / 2} \text {. }
$$

In the limit $\rho \ll 1$ and $\left|2 i \Delta^{2} / \Gamma\left(\omega+i D k^{2}\right)\right| \ll 1$ they get

$$
I_{2}=\frac{-i T}{\omega+i D k^{2}}\left[1-\frac{i \pi \Delta^{2}}{2 T\left(\omega+i D k^{2}\right)}\right] \text {. }
$$

and

$$
I_{4}=i \pi \Delta / 4\left(\omega+i D k^{2}\right) \text {. }
$$

Substituting Eqs. (41), (42), and (39) into Eq. (22), the dispersion relation becomes

$$
\omega^{2}-D k^{2}\left(\gamma+D k^{2}\right)+2 i \omega\left(\gamma+D k^{2}\right)=0 .
$$

The transverse eigenfrequency in the gapless regime $(\Delta<<\Gamma)$ is

$$
\omega=-i\left(\gamma+D k^{2}\right)\left(1 \pm\left(\gamma /\left(\gamma+D k^{2}\right)\right)^{1 / 2}\right) .
$$

Finally, for gaplcos oupcrconductoro ( $\rho, 1,1)$ the integrals becomo

$$
\begin{gathered}
T_{2}=\frac{-i T}{\omega+i D k^{2}}\left[1-\frac{i 2 \Delta^{2} / \Gamma}{\omega+i D k^{2}+2 i \Delta^{2} / \Gamma}\right] . \\
I_{4}=\frac{T}{2 \Delta} \frac{2 i \Delta^{2} / \Gamma}{\omega+i D k^{2}+2 i \Delta^{2} / \Gamma} \simeq \frac{T}{2 \Delta}
\end{gathered}
$$

and

$$
I_{5}=i I_{4}
$$

The resulting dispersion relation is 


$$
\omega^{2}-\gamma D k^{2}+i \omega\left(\gamma / 2+D k^{2}\right)=0 .
$$

The eigenfrequency for the transverse mode of gapless superconductors is purely imaginary.

$$
\omega=-i / 2\left[\left(\gamma / 2+D k^{2}\right)+\left(\left(\gamma / 2+D k^{2}\right)^{2}-4 \gamma D k^{2}\right)^{1 / 2}\right]
$$

The longitudinal mode of the pair-field susceptibility is associated with fluctuations in the order parameter modulus. The dispersion relation for modulus fluctuations derived from the Ginzburg-Landau equation and the Boltzmann equation is

$$
\alpha_{0}-3 \Delta^{2} \beta_{0}+\left(i \omega-D k^{2}\right) \psi^{\prime}(1 / 2+\rho) / 4 \pi T+I_{6} \omega / T=0
$$

Here $\alpha_{0}$ and $\beta_{0}$ are the $p$-dependent G1nzburg-Landau parameters:

$$
\alpha_{0}^{\prime}=\left(\left(T_{c}-T\right) / T_{c}\right)\left(1-\rho \psi^{\prime}(1 / 2+\rho)\right)
$$

and

$$
\beta_{0}=-\left(\psi^{\prime \prime}(1 / 2+\rho)+\rho \psi^{\prime \prime \prime}(1 / 2+\rho) / 3\right) /(4 \pi T)^{2} \text {. }
$$

In equilibrium

$$
\Delta^{2}=\alpha_{0} / e_{0}
$$

$I_{6}$ is an integral that can be calculated to leading order in $\Delta / T$ for the various limiting cases.

In the gap regime it is found that

$$
I_{6}=\int_{0}^{1} d \int^{2} d x\left(1-x^{2}\right) /\left(\omega\left(1-x^{4}\right)+i D k^{2}\left(1+x^{2}\right)^{2}\right) .
$$

The authors then evaluated $I_{6}$ in the high frequency limit 
$\left|\omega+i D k^{2}\right| \simeq \omega$ which. yields

$$
I_{6}=-\pi \Delta / 4 \omega
$$

Inserting this expression into Eq. (50) one gets the high frequency dispersion relation for the gap regime,

$$
-2 \dot{\alpha_{0}}+\left(i \omega-\mathrm{Dk}^{2}\right) \psi^{\prime}(1 / 2+\rho) / 4 \pi \mathrm{T}-\pi \Delta / 4 \mathrm{~T}=0
$$

This solucion is a purely diffusive mode given by

$$
\omega=-i\left(D k^{2}+\left(2 \Delta+16 T \alpha_{0} / \pi\right) / b\right)
$$

where $b=\psi^{\prime}(1 / 2+\rho) / \psi^{\prime}(1 / 2) \lesssim 1$. If one chooses the low frequency limit $\left|\omega+i D k^{2}\right| \simeq D k^{2}$ the integral $I_{6}$ becomes

$$
I_{6}=i \Delta / 2 \mathrm{Dk}^{2}
$$

Inserting Eq. (58) into Eq. (50) one gets the low frequency dispersion relation for the gap regime,

$$
-2 \alpha_{0}+\left(i \omega-D k^{2}\right) \psi^{\prime}(I / 2+\rho) / 4 \pi T+i \omega \Delta / 2 D k^{2} T=0
$$

The solution is

$$
\omega=-i\left(\mathrm{Dk}^{2}+16 \alpha_{\mathrm{o}} \mathrm{T} / \pi \mathrm{b}\right) /\left(1+4 \Delta / \pi \mathrm{bDk}{ }^{2}\right)
$$

One can show by numerical evaluation of $I_{6}$ that Eq. (60) represents a solution nearly identical to the exact solution for typical values of experimental parameters.

Ir the gapless regime $(\Delta<\Gamma)$ the integral $I_{6}$ is 


$$
I_{6}=-2 \Delta^{2} T\left(\psi^{\prime}(1 / 2+\rho) / \rho+\psi^{\prime \prime}(1 / 2+\rho)\right) /\left(\omega+i D k^{2}\right)(4 \pi T)^{2} .
$$

For weak pair-breaking $(\rho \ll 1)$ this becomes

$$
I_{6}=-\pi \Delta^{2} / 8 \Gamma\left(\omega+i D k^{2}\right)
$$

Inserting this expression into Eq. (50) one obtains the dispersion relation for the longitudinal mode in the gapless regime,

$$
-2 \alpha_{0}+\left(i \omega-D k^{2}\right) \psi^{\prime}(1 / 2+\rho) / 4 \pi T-\pi \omega \Delta^{2} / 3 \Gamma T /\left(\omega+i D k^{2}\right)=0
$$

The authors go to the high frequency limit obtaining

$$
\omega=-i\left(D k^{2}+\Delta^{2} / \Gamma\right)
$$

If one solves Eq. (63) exactly, however, one finds that there are two solutions,

$$
\omega=-i\left[D k^{2}+8 \alpha_{0} T / b \pi+\Delta^{2} / 2 \Gamma \pm\left(\left(8 \alpha_{0} T / b \pi+\Delta^{2} / 2 \Gamma\right)^{2}+\Delta^{2} D k^{2} / \Gamma\right)^{1 / 2}\right] .
$$

The upper sign gives the Orbach - Entin-Wohlmann-solution for $\mathrm{Dk}^{2} \rightarrow 0$. The lower sign represents a decaying solution similar to that found in the gap regime in the low frequency limit. It is of interest to examine the limit $\Delta \rightarrow 0$. To leading order Eq. (6.5) simplifies to

$$
\omega=-i\left(D^{2} \pm \Delta\left(D k^{2} / \Gamma\right)^{1 / 2}\right)
$$

provided $\Delta \ll \mathrm{Dk}^{2}, \Gamma$. If $\mathrm{Dk}^{2}$ is small enough; there will be a range $\dot{\mathrm{Dk}}^{2} \ll \Delta \ll \Gamma$. In this range Eq. (65) reduces to 


$$
\omega=\left\{\begin{array}{lc}
-i\left(D k^{2}+\Delta^{2} / \Gamma\right) & \text { (upper sign) } \\
-1.7 i \mathrm{Dk}^{2} & \text { (lower sign) }
\end{array}\right.
$$

The upper solution can again be identified with Eq. (64).

For gapless superconductors $(\rho>>1)$ the integral $I_{6}$ becomes

$$
I_{6}=i \pi^{2} T^{3} \Delta^{2} / 3\left(i \omega-D k^{2}\right) \Gamma^{4}
$$

For $\cap \gg 1,2 \alpha_{u}=\Delta^{2} / 6 \Gamma^{2}$ and $\psi^{\prime}(1 / 2+\rho)=1 / \rho$. Inserting this along with Eq. (68) into Eq. (50) one gets the dispersion relation for the longitudinal mode in gapless superconductors,

$$
-\Delta^{2} / 6 \Gamma^{2}+\left(i \omega-D k^{2}\right) / 2 \Gamma-i \pi \omega T^{2} \Delta^{2} / 3\left(i \omega-D k^{2}\right) \Gamma^{4}=0 .
$$

Again there are two solutions which simplify to

$$
\omega=\left\{\begin{array}{l}
-i\left(\mathrm{Dk}^{2}+\Delta^{2} / 3 \Gamma\right) \\
-i\left(\mathrm{Dk}^{2} \cdots\left(\mathrm{Dl}{ }^{2}+\Delta^{2} / \Gamma\right)\left(2 \pi^{2} \mathrm{~T}^{2} / \Gamma^{2}\right)\right)
\end{array}\right.
$$

Since Orbach - Entin-Wohlmann give only the upper solution, they predict a characteristic frcquency that increases linearly with $\alpha_{0}$. If the second solution in Eq. (70) coexists with first the characteristic frequencies may not be resolved experimentally. Furthermuse, if the second solution in Eq. (70) is the ont.y valid solution, one should measure a decreasing relaxation frequency in gapless superconductors in finite field.

In summary, for all three cases considered there are two possible solutions for the longitudinal mode. Both are predicted 
to be purely diffusive (the eigenfrequency is pure 1maginary). One solution increases while the other decreases from $\mathrm{Dk}^{2}$ as $\varepsilon$ increases. 
2. Pair-Field Susceptibility

In terms of the integrals $I_{2}, I_{4}, a$, and $I_{6}$ the longitudinal and transverse susceptibilities are given by

$$
x^{L}=(1 / N(0))\left(2 \alpha_{0}-(\pi b / 8 T)\left(i \omega-D k^{2}\right)-(\omega / T) I_{6}\right)^{-I}
$$

and

$$
X^{T}=(1 / N(0))\left(\omega^{2} /\left(2 \Delta^{2}\left(i \omega-D k^{2}\right)-\Delta T_{D k}^{2} / I_{4}\right)+a D k^{2}\right)^{-1}
$$

$N(0)$ is the electron density of seares di llie f'ermi level und $\mathrm{b}=\psi^{\prime}(1 / 2+\rho) / \psi^{\prime}(1 / 2)$. The imaginary part of the susceptibility is proportional to the measured excess current of a Josephson junction. Precise values for $\operatorname{Im}\left(X^{L}\right)+\operatorname{Im}\left(X^{T}\right)$ can be obtained by numerically integrating $I_{2}, I_{4}, a$, and $I_{6}$ and taking the imaginary part of the resulting numbers in Eqs. (71) and (72). Using the approximate values for the integrals, approximate analytical expressions for. $\operatorname{Im} \chi^{\mathrm{L}}$ and $\operatorname{Im} \chi^{\mathrm{T}}$ can be given.

krom Eqs. (29)-(31), (33), (55), (58), (71), and (72) we have in the gap regime:

$$
\begin{gathered}
\left.\operatorname{Im} \chi^{L}=\frac{4 \mathrm{~T}}{\pi \mathrm{bN}(0)} \frac{\omega}{\left(2 \Delta / \mathrm{b}+\mathrm{Dk}^{2}\right)^{2}+(1)^{2}}(\omega)>\mathrm{Dk}^{2}\right), \\
\operatorname{Im} \chi^{L}=\frac{4 \mathrm{~T}}{\pi \mathrm{bN}(0)} \frac{\omega /\left(1+4 \Delta / \pi \mathrm{b} n \mathrm{k}^{2}\right)}{\omega^{2}+\left(\left(\mathrm{Dk}^{2}+16 \mathrm{~T} \alpha_{0} / \pi \mathrm{b}\right) /\left(1+4 \Delta / \pi \mathrm{DDk}^{2}\right)\right)^{2}}
\end{gathered}
$$

for $\omega \varsigma \mathrm{Dk}^{2}$ and

$$
\operatorname{Im} \chi^{T}=2 \Delta^{2} \omega^{3} / \pi D k^{2} N(0)\left(\left(\omega^{2}-2 \Delta b D k^{2}\right)^{2}+\gamma^{2} \omega^{2}\right) \text {. }
$$


Here $\gamma=\pi b \Delta^{2} / 2 T$ and $b=\psi^{\prime}(1 / 2+\rho) / \psi^{\prime}(1 / 2)$ :

From Eqs. (40), (23), (39), (41), (42), (71), and (72) the gapless regime result is

$$
\operatorname{Im} \chi^{L}=\frac{4 T \Gamma}{\pi b \Delta^{2} N(0)} \frac{\omega \Gamma / \Delta^{2}}{1+\left(\omega \Gamma / \Delta^{2}\right)^{2}}
$$

for $\omega>\mathrm{Dk}^{2}$,

$$
\operatorname{Im} \chi^{L^{\prime}}=\frac{4 T \Gamma}{\pi b \Delta^{2} N(0)} \frac{\left(\omega^{2}+\left(D k^{2}\right)^{2}\right) \omega D k^{2}}{\left(\left(16 \alpha_{0} T \Gamma / \pi \Delta^{2}\right)\left(\omega^{2}+\left(D k^{2}\right)^{2}\right)\right)^{2}+\left(\omega D k^{2}\right)^{2}}
$$

for $\omega \leqslant \mathrm{Dk}^{2}$, and

$$
\operatorname{Im} \chi^{T}=\frac{2 \Delta^{2}\left(\gamma+D k^{2}\right)}{\gamma D k^{2} N(0)} \frac{\omega\left(\omega^{2}+\gamma D k^{2}+\left(D k^{2}\right)^{2}\right)}{\left(\omega^{2}-D k^{2}\left(\gamma+D k^{2}\right)\right)^{2}+4 \omega^{2}\left(\gamma+D k^{2}\right)^{2}} .
$$

For gapless superconductors the longitudinal mode from Eqs. (68) and (71) is

$$
\operatorname{Im} \chi^{L}=\omega\left(A+B D k^{2}\right) /\left(A^{2}+B^{2} \omega^{2}\right)
$$

where

$$
A=\omega^{2}-\left(D k^{2}\right)^{2}-\Delta^{2} D k^{2} / 3 \Gamma
$$

and

$$
B=2 D k^{2}+\Delta^{2}\left(1-1 / 2 \rho^{2}\right) / 3 \Gamma .
$$

The transverse mode in gapless superconductors from Eqs. (45)-(47) and (72) is

$$
\operatorname{Im} \chi^{T}=\frac{2 \Delta^{2}}{D k^{2} N(0)} \frac{\omega\left(2 \omega^{2}+4\left(D k^{2}\right)^{2}\right)}{\left(2 \omega^{2}-2 \gamma D k^{2}\right)^{2}+\omega^{2}\left(\gamma+D k^{2}\right)^{2}} .
$$


D. THEORY OF DINTER

Dinter ${ }^{24}$ provides a microscopic theory for the current-voltage characteristics for the range $0 \leqslant \omega \leqslant 3.5 \Delta$. In an earlier paper 38 he developed what he refers to as a generalized random-phase formalism. It consists of combining all possible types of twoparticle correlations in a $4 \times 4$ matrix scheme. All possible correlations between fluctuations of the complex order parameter and the quasiparticle density are included. Pair-breaking is included at the outset for values of $\rho \ll 1$. It is aiso assumed $\varepsilon \ll 1$. In this limit the order parameter is given by

$$
\Delta^{2}=8 \pi^{2} \mathrm{~T}_{c}^{2} \varepsilon / 7 \zeta(3)
$$

where $\zeta(3)=1.202$ is the Rieman zeta function of argument 3.

The surrent due to fluctuations, is caloulated to he

$$
I_{c x} \propto \operatorname{Im}\left[\frac{N(O)}{2}\left(\frac{1}{a-d}+\frac{1}{a+d+(b+c)^{2} /(f+g)}\right)\right],
$$

where the quantities $a \pm d, b+c$ and $f+g$ are calculated in terms of $\omega, \mathrm{Dk}^{2}, \Gamma, \Delta$ and $\mathrm{T}_{\mathrm{c}}$. The expressions contain elliptic integrals that can be approximated by analytic expressions for a couple special cases. The first and second terms in Eq. (84) are due to the longitudinal and transverse modes respectively.

The excess current due to the transverse mode can be calculated by evaluating the following quantities:

$$
(a+d) / N(0)=Y(b+c) / 2 \omega \Delta N(0)-S
$$


and

$$
\frac{\mathrm{f}+\mathrm{g}}{N(0)}=\frac{\mathrm{Dk}{ }^{2}}{4 \pi \sigma}-\frac{2 \omega \Delta}{\mathrm{Y}} \frac{\mathrm{b}+\mathrm{c}}{\mathrm{N}(0)}-\frac{4 \Delta^{2} \mathrm{~S}}{\mathrm{Y}}-\frac{\left.(\mathrm{Dk})^{2}\right)^{2}}{\mathrm{Y}}-\frac{i \omega \mathrm{Dk}}{\mathrm{Y}} \frac{\sigma(\omega, \mathrm{k})}{\sigma}
$$

The term $\mathrm{Dk}^{2} / 4 \pi \sigma$ in $\mathrm{Eq}$. (86) may be neglected for frequencies of interest. $\left(\mathrm{Dk}^{2}\right)^{2} / Y$ has been added to reproduce correctly the limit in which $\Delta \rightarrow 0 .^{38}$

$$
\begin{aligned}
& \mathrm{Y} \equiv \omega^{2}-\left(D k^{2}\right)^{2} \\
& \mathrm{~S} \simeq \frac{\mathrm{Dk}^{2}}{8 \mathrm{~T}}\left[\pi+\frac{2 \omega}{\omega+2 \Delta} \mathrm{K}\left(\frac{2(2 \omega \Delta)^{1 / 2}}{\omega+2 \Delta}\right)-\frac{4 i \omega}{\omega+2 \Delta} \mathrm{K}\left(\frac{2 \Delta-\omega}{2 \Delta+\omega}\right) \Theta(2 \Delta-\omega)\right] \\
& \frac{\sigma(\omega, k)}{\sigma} \simeq \frac{\pi \Delta^{2}}{2 T} \frac{i}{\omega}+1-\left[\frac{\omega+2 \Delta}{4 T} E\left(\frac{2 \Delta-}{2 \Delta+\omega}\right)-\frac{\Delta}{T} k\left(\frac{2 \Delta-\omega}{2 \Delta+\omega}\right)\right] \theta(2 \Delta-\omega) \\
& +i\left[\frac{\omega+2 \Delta}{8 T} E\left(\frac{2(2 \Delta \omega)^{1 / 2}}{2 \Delta+\omega}\right)-\frac{\omega-2 \Delta}{8 T} K\left(\frac{2(2 \Delta \omega)^{1 / 2}}{2 \Delta+\omega}\right)\right] \\
& \frac{b+c}{N(0)} \frac{\pi \omega^{2} \Delta}{4 T\left[Y\left(4 \Delta^{2}-Y\right)\right]^{1 / 2}} \theta(2 \Delta \omega-Y)+\frac{\omega^{2} \Delta D k^{2}}{4 T Y(\omega+2 \Delta)}\left[K\left(\frac{2(2 \Delta \omega)^{1 / 2}}{\omega+2 \Delta}\right)\right. \\
& \left.+\frac{Y-2 \omega \Delta}{Y+2 \omega \Delta} \Pi\left(\frac{8 \omega \Delta Y}{(2 \Delta \omega+Y)^{2}}, \frac{2(2 \Delta \omega)^{1 / 2}}{\omega+2 \Delta}\right)\right] \\
& +\frac{i \omega \Delta D k^{2}}{2 \operatorname{TY}(\omega+2 \Delta)} \theta(2 \Delta-\omega) \quad\left\{-K\left(\frac{2 \Delta-\omega}{2 \Delta+\omega}\right)\right. \\
& \left.+\frac{\mathrm{Y}^{2}-4 \omega^{2} \Delta^{2}}{\left(\Pi k^{2}\right)^{2}\left(Y-1 \Delta^{2}\right)}\left[-K\left(\frac{2 \Delta-\omega}{2 \Delta+\omega}\right)+\Pi\left(\frac{\left(\mathrm{Dk}^{2}\right)^{2}\left(4 \Delta^{2}-\mathrm{Y}\right)}{\mathrm{Y}(\omega+2 \Delta)^{2}}, \frac{2 \Delta-\omega}{2 \Delta+\omega}\right)\right]\right] \\
& +\frac{i \pi \omega^{2} \Delta}{4 T\left[Y\left(Y-4 \Delta^{2}\right)\right]^{1 / 2}} \theta(\omega-2 \Delta)
\end{aligned}
$$


Here $K, E$, and $\Pi$ are complete elliptic integrals of the first, second and third kinds respectively. Implicit in the above expressions and those to follow is $\mathrm{Dk}^{2} \ll 2 \Delta$. It should be noted that the expressions shown above do not contain any dependence on $\Gamma$. The depencence on $\Gamma$ for $\dot{\Gamma} \ll \mathrm{T} / 2 \pi$ is weak and ${ }^{\circ}$ is lost in taking the extreme gap $(\Delta \gg \Gamma)$ limit.

In order to compare to other theories one can express the elliptic integrals analytically for the frequency range $D k^{2} \ll \omega \ll 2 \Delta$. Then the transverse mode susceptibility is

$$
\begin{aligned}
& -\frac{N(0)}{2} \frac{1}{(a+d)+(b+c)^{2} /(f+g)}= \\
& \quad \frac{4 T}{\pi D k^{2}} \frac{2 \Delta D k^{2}-\left(\pi \Delta^{2} / 2 T\right)}{-\omega^{2}+2 \Delta D k^{2}-i \omega\left[\left(\pi \Delta^{2} / 2 T\right)+\left(2 D k^{2} / \pi\right) \ln (4 \Delta / \omega)\right]}
\end{aligned} .
$$

The quantity a - d in the longitudinal susceptibll1ty is calculated piece-meal.

$$
\begin{aligned}
& \operatorname{Re} \frac{\mathrm{a}-\mathrm{d}}{\mathrm{N}(0)}=-\frac{7 \zeta(3) \Delta^{2}}{4 \pi^{2} \mathrm{~T}^{2}}-\frac{\pi \mathrm{Dk}}{8 \mathrm{~T}}-\frac{\pi \omega}{16 \mathrm{~T}}\left(\frac{4 \Delta^{2}-\mathrm{Y}}{\mathrm{Y}}\right)^{1 / 2} \\
& \quad-\frac{\mathrm{Dk}^{2} \omega}{8 \mathrm{~T}(\mathrm{Y}+2 \omega \Delta)}\left[4 \Delta \mathrm{K}\left(\frac{2(2 \Delta \omega)^{1 / 2}}{2 \Delta+\omega}\right)-\frac{\left(4 \Delta^{2}-\mathrm{Y}\right)(\mathrm{Y}-2 \Delta \omega)}{\mathrm{Y}(\omega+2 \Delta)}\right. \\
& \left.\quad \mathrm{x} \Pi\left(\frac{(\mathrm{Y}+2 \Delta \omega)^{2}}{\mathrm{Y}(\omega+2 \Delta)^{2}}, \frac{\left.2(2 \omega \Delta)^{1 / 2}\right)}{\omega+2 \Delta}\right)\right]
\end{aligned}
$$

for $0 \leq \omega \leq\left[4 \Delta^{2}-\left(D k^{2}\right)^{2}\right]^{1 / 2}$. 


$$
\begin{aligned}
& \operatorname{Re} \frac{a-d}{\mathbb{N}(0)}=-\frac{7 \zeta(3) \Delta^{2}}{4 \pi^{2} T^{2}}-\frac{\pi D k^{2}}{8 T}-\frac{\omega D k^{2}}{8 T Y(\omega+2 \Delta)}\left[\left(4 \Delta^{2}+Y\right) K\left(\frac{2(2 \omega \Delta)^{1 / 2}}{\omega+2 \Delta}\right)\right. \\
& \left.\quad+\left(4 \Delta^{2}-Y\right) \frac{Y-2 \Delta \omega}{Y+2 \Delta \omega} \Pi\left(\frac{8 \omega \Delta Y}{(2 \Delta \omega+Y)^{2}}, \frac{2(2 \omega \Delta)^{1 / 2}}{2 \Delta+\omega}\right)\right]
\end{aligned}
$$

for $\left[4 \Delta^{2}-\left(D k^{2}\right)^{2}\right]^{1 / 2} \leq \omega \leq \mathrm{T}$.

$$
\begin{aligned}
& \operatorname{Im} \frac{a-d}{N(0)}=\frac{\omega}{4 \mathrm{TDk}^{2}(\omega+2 \Delta)}\left[\left(4 \Delta^{2}-\omega^{2}\right) \mathrm{K}\left(\frac{2 \Delta-\omega}{2 \Delta+\omega}\right)\right. \\
& \left.\quad-\frac{\left(4 \omega^{2} \Delta^{2}-\mathrm{Y}^{2}\right)}{\mathrm{Y}} \pi\left(\frac{(\mathrm{Dk})^{2}\left(4 \Delta^{2}-\mathrm{Y}\right)}{\mathrm{Y}(\omega+2 \Delta)^{2}}, \frac{2 \Delta-\omega}{2 \Delta+\omega}\right)\right] \Theta(2 \Delta-\omega) \\
& \quad+\frac{\pi \omega\left(\mathrm{Y}-4 \Delta^{2}\right)^{1 / 2}}{8 \mathrm{TY} \mathrm{Y}^{1 / 2}} \Theta(\omega-2 \Delta) .
\end{aligned}
$$

The excess current due to the longitudinal mode is proportional to $\operatorname{Im}(\mathrm{N}(0) /(\mathrm{a}-\mathrm{d}))$. This function consists of a maximum at a low frequency and a second maximum at $\mathrm{V} \simeq 1.25 \Delta$.

Dinter claims finite $\Gamma$ is necessary to avoid a logrithmic divergence of the longitudinal structure function at $\omega=0$. He axamines the $w \lll \Gamma \leftrightarrow \Delta \lll T$ limit in detail. With $B=\Gamma / \Delta$, he Finds:

$$
-\frac{a-d}{N(0)} \simeq \frac{\pi D k}{8 T}+2 \varepsilon-i \omega \frac{\pi}{8 T} f_{1}(\beta)-i \omega \frac{\Delta}{D k^{2}} \frac{\pi}{8 T} f_{2}(\beta),
$$

where

$$
f_{1}(\beta)=\frac{2}{\pi}\left[\frac{\left(1-\beta^{2 / 3}\right)^{1 / 2}}{\beta^{1 / 3}}+\tan ^{-1}\left(\frac{\beta^{1 / 3}}{\left(1-\beta^{2 / 3}\right)^{1 / 2}}\right)\right]
$$

and 


$$
\begin{aligned}
f_{2}(\beta) & =\frac{4}{\pi}\left\{\ln \left(\frac{1+\left(1-\beta^{2 / 3}\right)^{1 / 2}}{\beta^{1 / 3}}\right)+\frac{1}{2 \beta} \tan ^{-1}\left(\frac{\beta^{1 / 3}}{\left(1-\beta^{2 / 3}\right)^{1 / 2}}\right)\right. \\
& \left.-\frac{\left(1-\beta^{2 / 3}\right)^{1 / 2}}{2 \beta^{2 / 3}}\left[\left(1-\beta^{2 / 3}\right)^{2}+\beta^{4 / 3}\right]\right\} .
\end{aligned}
$$

When Eq. (95) is evaluated at $\beta=1$ (the boundary between the gapless and gap regime) one gets

$$
-\frac{\mathrm{a}-\mathrm{d}}{\mathrm{N}(0)}=\frac{\mathrm{Dk}^{2} \pi}{8 \mathrm{~T}}+2 \varepsilon-\frac{i \omega \pi}{8 \mathrm{~T}}-\frac{i \omega \Delta \pi}{8 \mathrm{TDk}}{ }^{2} .
$$

The same quantity calculated in the gapless regime ${ }^{39}$ and evaluated at $\Delta=\Gamma$ is

$$
-\frac{a-d}{N(0)}=\frac{D k^{2}}{8 T}+2 \varepsilon-\frac{i \omega \pi}{8 T}-\frac{i \omega \Delta \pi}{8 T\left(-i \omega+D k^{2}\right)} .
$$

Although the first expression is deduced from an equation that was derived for $\beta \ll 1$ and the latter from an equation derived for $\beta \ll 1$, Dinter proposes that the discrepancy at $\beta=1$ should be resolved by substituting $-i \omega+\mathrm{Dk}^{2}$ for $\mathrm{Dk}^{2}$ in the denominator of the last term in Eq. (98). As the expression for $\beta \ll 1$ was derived under the assumption that $\omega \ll \mathrm{Dk}^{2}$ there should be no inconsistency in adding $-i \omega$ to $\mathrm{Dk}^{2}$. But one must be suspicious of the $\mathrm{Dk}^{2} \rightarrow 0$ result once this is done. Setting $\mathrm{Dk}^{2}=0$ Dinter finds the relaxation frequency from $-(a-d) / N(0)=0$ in Eq. (98). Neglecting $2 \mathrm{f}$

$$
w=-i \Delta f_{2}(\beta) / f_{1}(\beta) .
$$


This gives a relaxation frequency of $\Delta$ at $\beta=1$. One.can easily solve for $\omega$ without taking $k=0$ limit or neglecting the $2 \varepsilon$ term. Solving for $\omega$ before making the $-i \omega+\mathrm{Dk}^{2}$ substitution one finds from Eq. (98)

$$
\omega=-i \frac{D k^{2}+16 \varepsilon T / \pi}{f_{1}(B)+\Delta f_{2}(B) / D k^{2}} \cdot \text { (Dinter) }
$$

Compare this to the low-frequency Entin-Wohlman - Orbach result in Eq. $(60)$. For $\rho=0$

$$
\omega=-i \frac{\mathrm{Dk}^{2}+16 \varepsilon \mathrm{T} / \pi}{1+4 \Delta / \pi \mathrm{Dk}^{2}} \cdot \text { (Entin-Wohlman - Orbach) }
$$

If one makes the $\mathrm{Dk}^{2} \rightarrow-i \omega+\mathrm{Dk}^{2}$ substitution in $\mathrm{Eq} \cdot(98)$ as suggested before, but then solve for $\omega$ exactly without taking $k=0$ limit or neglecting $\varepsilon$ one gets

$$
\omega=-i D k^{2}\left\{B \pm\left[B^{2}-\left(1+16 \varepsilon T / \pi D k^{2}\right) / 2 f_{1}(B)\right]^{1 / 2}\right\} \text {, }
$$

where

$$
B=\left[1+16 \varepsilon \mathrm{ET} / \pi D k^{2}+f_{1}(\beta)+f_{2}(\beta) \Delta / D k^{2}\right] / 2 f_{1}(\beta)
$$

One gets a high frequency solution if the " + " sign is taken:

$$
i \omega \simeq \Delta \mathrm{f}_{2}(\beta) / \mathrm{f}_{1}(\beta)+\mathrm{Dk}^{2}\left(1+1 / \mathrm{f}_{1}(\beta)\right)+16 \varepsilon \mathrm{T} / \pi \mathrm{f}_{1}(\beta) .
$$

Taking the minue gign in Eq. (103) glves the low frequency solution

$$
i \omega \simeq \frac{D k^{2}+16 \varepsilon T / \pi}{2\left(1+f_{1}(\beta)+16 \varepsilon T / \pi D k^{2}+\Delta f_{2}(\beta) / D k^{2}\right)}
$$

The high frequency solution is not significantly different from that 
calculated before. The low frequency solution, however, is much smaller. At $\beta=1$, for example,

$$
\omega(\beta=1)=-i\left(D k^{2}+16 \varepsilon T / \pi\right) / 2\left(2+\Delta / D k^{2}+16 \varepsilon T / \pi D k^{2}\right) .
$$

This is more than a factor of two smaller than the frequency obtained from Eq. (101).

A closing thought here is can it mean anything to find a solution $\omega \simeq \Delta$ when one is using $\omega \rightarrow 0$ limit to do the calculation? If only one of the above solutions is valid it is probably Eq. (101). That is one should not make the replacement $\mathrm{Dk}^{2} \rightarrow-i \omega+\mathrm{Dk}^{2}$. Doing it seems only to introduce an extraneous root at high frequencies. This is not to say there is no solution at high frequencies--only that such a solution might not be found in a low frequency limit calculation. 
E. THEORY OF SCHÖN AND AMBEGAOKAR

This theory includes the effects of a superconducting transport current on the dynamics of the order parameter fluctuations for dirty superconductors at temperatures near $T_{C}$. The effect of pairbreaking for $\Gamma \ll T / 2 \pi$ is also included.

The methods developed by Schön in an earlier work ${ }^{15}$ were extended to modify the work of Ambegaokar ${ }^{40}$ which considered the effects of an impressed pair monentum. A time-dependent GinzburgLandau equation for the order parameter and a Boltzman rate equation for the quasiparticle distribution function are derived. General solutions are given in terms of elliptic integrals, but analytic solutions are possible only in certain limiting cases. In particular solutions are given for $\mathrm{Dk}^{2} \ll \omega$ only. Special cases include negligible inelastic phonon scattering of electrons $\left(1 / \tau_{E} \ll \omega\right)$ as well as electron-phonon dominated relaxation $\left(1 / \tau_{E}>\omega\right)$ for both $q=0$ (no transport current) and $q \neq 0$. For all cases it is assumed that

$$
\Delta_{0}^{2} / T \ll\left|1 / \tau_{E}-i \omega\right|
$$

where

$$
\Delta_{0}^{2}=\left(1-q^{2} / 3 q_{c}^{2}\right) \Delta^{2}
$$

Here $q_{c}$ is the critical pair momentum given by

$$
q_{c}^{2}=1 / 3 \xi^{2}(T)
$$

and $\Delta$ is the $\mathrm{q}=0$ order parameter. 
From the Fourier transformed time dependent Ginzburg-Landau equation, which has been linearized in the space and time-dependent deviations from equilibrium of the complex order-parameter, one gets the following set of simultaneous equations for $\delta \Delta^{L}(r, t)$ (deviation in the real part of $\Delta(r, t)), \delta \Delta^{T}(r, t)$ (deviation in the imaginary part of $\Delta(r, t)$ ) and $e \Phi(r, t)$ (the electric potential).

$$
\begin{aligned}
& {\left[-i \omega 2 \Delta_{\Omega} \tau \zeta+D k^{2}+16 \varepsilon \mathrm{T} / \pi\right] \delta \Delta^{\mathrm{L}}+\left(1+2 i \omega \mathrm{Y}_{2\urcorner} / \pi\right) 2 i D k q \delta \Delta^{\mathrm{T}}=0} \\
& {\left[-i \omega n / 2 \Delta_{0} \tau+D \mathrm{k}^{2}\right] \delta \Delta^{\mathrm{T}}-(n / \tau) e \Phi-\left(1+2 i \omega \mathrm{Y}_{22} / \pi\right) 2 i D k q \delta \Delta^{\mathrm{L}}=0} \\
& -i \omega n \delta \Delta^{\mathrm{T}}-\left(2 \Delta_{0} \eta+\frac{8 \mathrm{TDk} / \pi}{1 / \tau+D k^{2}}\right) \operatorname{el}-\frac{4}{\pi} \mathrm{Dkq} \omega \mathrm{Y}_{12} \delta \Delta^{\mathrm{L}}=0
\end{aligned}
$$

Here $Y_{12}$ and $Y_{22}$ are functions which contain elliptic integrals,

$$
\begin{aligned}
& 11=\sqrt{2 y \uparrow} / v{ }_{1+Y / 2 L_{n}^{2}}^{2} \tau, \\
& \zeta=\sqrt{\lambda+\left(Y / \Delta_{0}\right)^{2-}}-Y / \Delta_{0}+1 / 2 \Delta_{0}{ }^{\prime}, \\
& 1 / \tau=-i \omega+1 / \tau_{E}, \\
& \gamma=-i \omega / 2+\Gamma,
\end{aligned}
$$

allu

$$
\Gamma=1 / 2 \tau_{E}+\text { other depairing energies. }
$$




\section{Solutions for Zero Transport Current}

For $\mathrm{q}=0$ Eqs. (111) - (113) reduce to

$$
\begin{aligned}
& \left(-i \omega 2 \Delta \tau \zeta+D k^{2}+16 \varepsilon T / \pi\right) \delta \Delta^{L}=0, \\
& \left(-i \omega n / 2 \Delta \tau+D k^{2}\right) \delta \Delta^{T}-(\eta / \tau) e \Phi=0,
\end{aligned}
$$

and

$$
-i \omega n \delta \Delta^{T}-\left(2 \Delta \eta+8 T D k^{2} / \pi\left(1 / \tau+D k^{2}\right)\right) e \Phi=0
$$

The dispersion relation for the longitudinal mode is decoupled from that of the transverse mode. Solving Eq. (119) in the strong electron-phonon scattering limit, $\left(I / \tau_{E} \gg \omega\right)$, one gets the characteristic frequency of the longitudinal mode

$$
i \omega=\left(D k^{2}+16 \varepsilon T / \pi\right) /\left(1-2 \tau_{E} \Gamma+2 \Delta \tau_{E}\left(1+(\Gamma / \Delta)^{2}\right)^{1 / 2}\right) .
$$

The limiting values of this frequency are

$$
i \omega=\mathrm{Dk}^{2}+16 \varepsilon \mathrm{T} / \pi \quad \text { (gapless regime). }
$$

and

$$
i(t)=\left(n k^{2}+16 \varepsilon_{c} T / \pi\right) / 2 \tau_{E} \Delta \quad \text { (gap regime) } .
$$

Note that the frequency in Eq. (123a) is the well-known GinzburgLandau frequency for superconductors below the critical temperature. Turning now to the limit $1 / \tau_{E} \ll \omega E q$. (119) glves the longitudinal mode eigen-frequency for negligible electron-phonon scattering:

$$
i \omega=2 \Gamma-\left(\left(2 \Gamma-D k^{2}-16 \varepsilon T / \pi\right)^{2}-(2 \Delta)^{2}\right)^{1 / 2} .
$$

The limiting values of Eq. (1.24) are 


$$
\mathrm{i} \omega=\mathrm{Dk}^{2}+\Delta^{2} /\left(\Gamma-\mathrm{Dk}^{2} / 2\right) \quad \text { (gapless regime) }
$$

and

$$
i \omega=2 \Gamma \pm i 2 \Delta \quad \text { (gap regime). }
$$

One again finds a purely diffusive mode in the gapless regime. One finds, however, that the mode is not diffusive in the extreme gap regime. Eq. (125b) predicts a resonant frequency of $2 \Delta$ with damping due to pair-breaking. Since $\Delta \gg \Gamma$ this should be manifested as a peak at $V=\Delta$ in the excess current-voltage characteristics.

To highlight the effect of electron-phonon scattering we plot in Fig. (1) the imaginary frequency (dotted line) predicted by Eq. (122) as a function of temperature. Superimposed we plot the imaginary part (dashed line) and real part (solid line) of the frequency in Eq. (124). The parameters used are $\mathrm{Dk}^{2}=10 \mu \mathrm{eV}$,

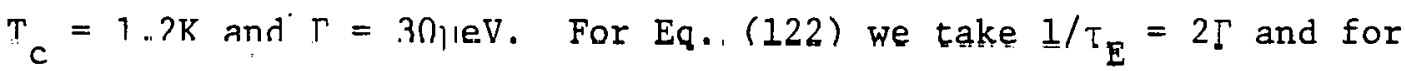
E'q. (124) we rake $1 / \tau_{E}=0$.

The group velocity of this mode $1 \mathrm{~s} \mathrm{D}^{2} \mathrm{k}^{3} / \Delta$. For cledil supeiconductors $\left(D \simeq 10^{4} \mathrm{~cm}^{2} / \mathrm{sec}\right)$ and short wavelengths $\left(\mathrm{k} \sim 10^{4} \mathrm{~cm}^{-1}\right)$ the mode velocity can be large $\left(\sim 10^{9} \mathrm{~cm} / \mathrm{sec}\right)$. Since the decay time can be as long as $\tau_{E}\left(\sim 10^{-7} \mathrm{sec}\right)^{41}$ it is possible that propagation of this mode can be directly observed. 42

The transverse mode dispersion relation 1 s obtafned by cumbining Eqs. (120) and (121) in such a way as to eliminate the dependence on electric potential. For zero supercurrent one finds

$$
i \omega / \tau-\left(\pi \Delta^{2} / 2 \mathrm{~T}\right)\left(I / \tau+\mathrm{Dk}^{2}\right)-2 \Delta \mathrm{Dk}^{2} / \eta=0 \text {. }
$$




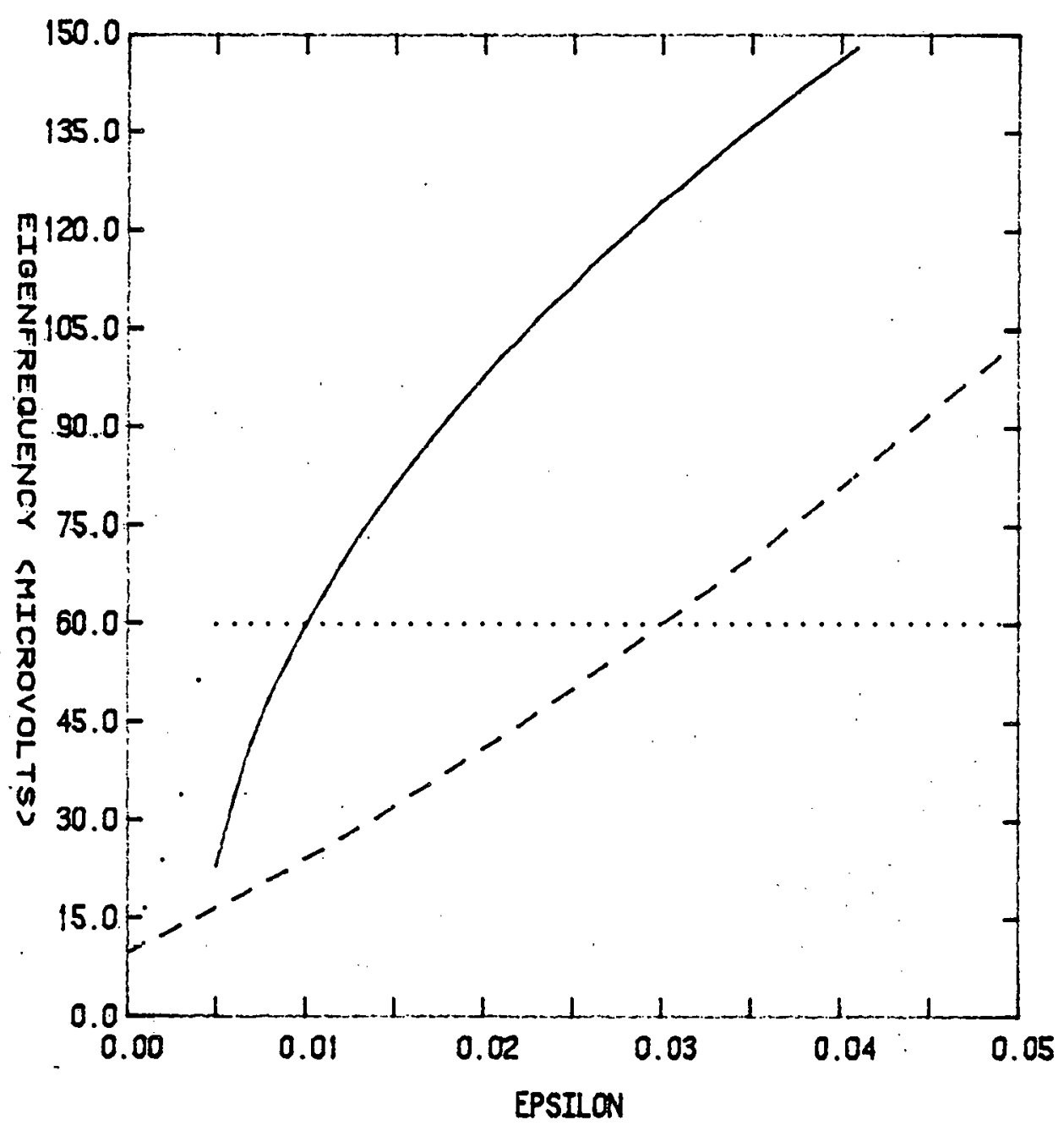

Figure 1. Plot of the Iongitudinal mode eigenfrequency for the Schön-Ambegaokar theory for $\mathrm{T}_{\mathrm{C}}=1.2 \mathrm{~K}, \mathrm{Dk}^{2}=10 \mathrm{\mu eV}$, and $\Gamma=30 \mu \mathrm{eV}$. The dashed line is for $1 / \tau_{E}=2 \Gamma^{\prime}$. The solid and dotted lines are the real and imaginary frequencies respectively for $1 / \tau_{E}=0$. 
In the case of superconductors with strong. inelastic electronphonon scattering, $\eta$ is approximately frequency independent. Replacing $1 / \tau$ by $1 / \tau_{E}$, Eq. (126) can be solved for $i \omega$ directly:

$$
i(1)=\left(\pi \Delta^{2} / 2 T\right)\left(1+\tau_{E} D k^{2}\right)+2 \Delta D k^{2} \tau_{E} / n \text {, }
$$

where $\eta \simeq 2 \Delta \tau_{E} /\left(1+\left(2 \Delta \tau_{E}\right)^{2}\right)^{1 / 2}$.

, In the high frequency regime one can put $1 / \tau=-i \omega$ and $\eta=1$. Then in the extreme gap regime the transverse mode dispersion relation becomes

$$
(i \omega)^{2}-i \omega\left(\pi \Delta^{2} / 2 T\right)+\left(\pi \Delta^{2} / 2 T+2 \Delta\right) D k^{2}=0 .
$$

The solution can be written

$$
i \omega=\dot{\omega}_{D} \pm\left(\omega_{D}^{2}-c^{2} k^{2}\right)^{1 / 2} .
$$

This is a propagating mode with velocity $c$ given by

$$
c^{2}=2 \Delta D(1+\pi \Delta / 4 T)
$$

and damping $\omega_{D}$ given by

$$
\omega_{D}=\pi \Delta^{2} / 4 \mathrm{~T}
$$

Corrections due to finite $\mathrm{Dk}^{2}$ are of order $\frac{\Delta}{\mathrm{T}}$ In $\frac{\Delta}{\omega}$ for the resonant frequency $c k$, while the damping is increased by an amount of order $\mathrm{Dk}^{2}$. Furthermore, pair-breaking effects due to paramagnetic impurities increase the damping by $\sim \Gamma / 2$ for small $\Gamma$. With these modifications Eqs. (130) and (131) would look like 


$$
c^{2}=2 \Delta D\left[1+(\pi \Delta / 4 T)\left(1+o\left(\ln \frac{\Delta}{\omega}\right)\right)\right]
$$

and

$$
\omega_{D}=\pi \Delta^{2} / 4 T+\sim D k^{2}+\sim \Gamma / 2
$$

in the gap regime $(\Gamma \ll \Delta \ll T)$.

In the extreme gapless regime $(\Delta<\Gamma)$ one finds $2 \Delta / \eta \simeq 1 / \tau$ $+\Delta^{2} / \Gamma$. Substituting this into Eq. (126) and taking the high frequency $(1 / \tau=-i \omega)$ limit, the transverse dispersion relation becomes

$$
(i \omega)^{2}-i \omega\left(D k^{2}+\pi \Delta^{2} / 2 T\right)+\left(\Delta^{2} \mathrm{Dk}^{2} / \Gamma\right)(1+\pi \Gamma / 2 T)=0 .
$$

The solution reduces to

$$
i \omega=D k^{2}-\Delta^{2} / \Gamma
$$

for $\Delta \ll \Gamma \ll T$ and $D k^{2} \gg \Delta^{2} / T$. Although there is a second solution in this limit given by $i \omega=\Delta^{2} / \Gamma$, it is known that $i \omega=D k^{2}$ at the critical temperature. We therefore take Eq. (135) to be the correct solution. 
2. The Current-Carrying State

If a current is passed through the superconductor, the superconducting electrons will have a finite momentum defined by the current density and superconducting electron density. One effect of finite momentum $q$ is to couple the transverse and longitudinal modes. As a consequence the coupled Eqs. (111) - (113) have a nontrivial solution only if the corresponding secular determinant vanishes. The characteriśtic equation is

$$
\begin{gathered}
\mathrm{k}^{2}\left[\left(-i \omega 2 \Delta_{0} \tau \zeta+\mathrm{Dk}^{2}+16 \beta \Delta_{0}{ }^{2} \mathrm{~T} / \pi\right)\left(-i \omega n / 2 \Delta_{0} \tau+\pi \Delta_{0} n / 4 T \tau+\mathrm{Dk}^{2}\right)\right. \\
\left.-4 \mathrm{Dq}^{2}\left(1+\frac{2 i \omega}{\pi} \mathrm{Y}_{22}\right)^{2}\left(\left(\pi \Delta_{0} / 4 \mathrm{~T}\right) \eta / \tau+D k^{2}\right)\right]+0
\end{gathered}
$$

The term containing $\mathrm{Y}_{12}$ in Eq. (113) is of order $\Delta_{\mathrm{o}} / \mathrm{T}$ and is therefore neglected.

The gange transformation is always present as a $k^{2}=0$ sntution of Eq. (136). This corresponds to the arbitrary choice of a reterence phase. 'lhe remaining part of Eq. (136) reduces in the limit $1 / \tau \rightarrow-i \omega$ to

$$
\begin{aligned}
(i \omega)^{2}- & i \omega\left[\left(\pi \Delta_{0}^{2} / 2 \mathrm{~T}\right)\left(1-\mathrm{Dq}^{2} /\left(2 \Delta_{0}+\mathrm{Dk}^{2}\right)\right)\right] \\
& +2 \Delta_{0} \mathrm{Dk}^{2}\left(1-\mathrm{Dq}^{2} /\left(2 \Delta_{0}+\mathrm{Dk}^{2}\right)\right)=0 .
\end{aligned}
$$

In this limit $\eta=1, \zeta=1$ and $Y_{22} \simeq i \pi / 4 \omega$ for $i \omega \gg 2 \Gamma$. According to Eq. (137) both the resonant frequency and the damping are reduced when there is a finite pair monentum $q$.

$$
\mathrm{C}^{2} \mathrm{k}^{2}=2 \Delta_{\mathrm{O}} \mathrm{Dk}{ }^{2}\left(1-\mathrm{Dq}^{2} /\left(2 \Delta_{\mathrm{O}}+\mathrm{Dk}^{2}\right)\right)
$$




$$
\omega_{D}=\left(\pi \Delta_{0}^{2} / 4 T\right)\left(1-D q^{2} /\left(2 \Delta_{0}+D k^{2}\right)\right)
$$

But one effect of pair-breaking due to supercurrent is to increase the damping by about $\mathrm{Lq}^{2} / 4$ (see Eq. (133)). Since this effect is of order $T / \Delta_{0}$ larger than the q-correction in Eq. (139) the damping should instead by given by

$$
\omega_{D}=\pi \Delta_{0}^{2} / 4 \mathrm{~T}+\mathrm{Dq}^{2} / 4
$$

Furthermore $\Delta_{0}^{2}=\Delta^{2}\left(1-q^{2} / 3 q_{c}^{2}\right)$ where $3 q_{c}^{2}=\xi^{-2}(T)$ and in the dirty limit

$$
\xi^{2}(\mathrm{~T})-\pi \mathrm{D} / 8 \mathrm{~T}_{c} \varepsilon
$$

Combining this with Eq. (140) and noting that $\Delta^{2}=8 \pi^{2} T^{2} \varepsilon / 7 \zeta(3)$ we get

$$
\omega_{D}=\frac{\pi \Delta^{2}}{4 T}-\left(\frac{\pi^{4}}{28 \zeta(3)}-1 / 4\right) D q^{2}
$$

Thus the damping is decreased by 2.64 times $\mathrm{Dq}^{2}$ when the q-dependence of $\Delta_{0}$ is taken into account.

By adding a normalized driving force term $(1 / 2 \mathrm{~N}(0))$ to the righthand sides of Eqs. (111) and (112) one obtains the pair-field susceptibility. The longitudinal and transverse parts are given by

$$
X^{L}=\frac{8 T}{2 \pi N(0)} \frac{1}{D k^{2}} \frac{(T) D k^{2}-i D k q(R)}{(L)(T)-D q^{2}(R)}
$$

and

$$
X^{T}=\frac{3 T}{2 \pi N(U)} \frac{1}{D k^{2}} \frac{(L)+i D k q(R)}{(L)\left(L^{\prime}\right)-V q^{2}(K)}
$$


where

$$
\begin{aligned}
& (L)=-i \omega 2 \Delta_{0} \tau \zeta+\mathrm{Dk}^{2}+16 \mathrm{~T} \varepsilon / \pi, \\
& (\mathrm{T})=-i \omega n / 2 \Delta \tau+\pi \Delta \eta / 4 \mathrm{~T} \tau+\mathrm{Dk}^{2},
\end{aligned}
$$

and

$$
(R)=\pi \Delta \eta / 4 T \tau+D k^{2} \text {. }
$$

Eqs. (143) and (144) are valid for $\omega \gg 2 \Gamma$, but can be extended to includo the gapleoo regime by maleing the oubotitution

$$
q \rightarrow 2 q\left(1+2 i \omega Y_{.22} / \pi\right)
$$

Schön and Ambegaokar have evaluated $\mathrm{Y}_{22}$ in several limits. For $\gamma=1 / 2 \tau$ (that is $\omega>>2 \Gamma$ )

$$
Y_{22} \simeq \pi \tau / 4\left(1+\left(1 / 2 \Delta_{0} \tau\right)^{2}\right)
$$

The corrections are of order $|\ln | \Delta_{0} \tau\left|/\left(\Delta_{0} \tau\right)\right|$. The the gapless regime $\left(|\gamma| \gg \Delta_{0}\right), Y_{22}$ becomes

$$
Y_{Z 2}= \begin{cases}\pi \Delta_{0}^{4} \tau^{2} / 16 \gamma^{3} & \text { for } \Delta_{0}^{2} \ll|\gamma / \tau|, \\ \pi \Delta_{0}^{2} \tau / 16 \gamma^{2} & \text { for } \Delta_{0}^{2} \gg|\gamma / \tau| .\end{cases}
$$


3. Mode Softening

Eq. (136) can be solved in the low frequency regime yielding

$$
\begin{aligned}
& \omega_{ \pm}=\frac{i}{2}\left[\frac{2 \Delta_{0} \tau_{E}}{\eta}\left(\omega_{R}^{T}+D k^{2}\right)+\frac{1}{2 \Delta_{o} \tau_{E} \zeta}\left(\frac{16 \mathrm{~T}}{\pi} \beta \Delta_{0}^{2}+D k^{2}\right)\right] \\
& \pm \frac{i}{2}\left[[\ldots]^{2}-\frac{4}{n \zeta}\left(\omega_{R}^{T}+D k^{2}\right)\left(\frac{16 T}{\pi} \beta \Delta_{0}^{2}+D k^{2}-D q^{2}\right]^{1 / 2},\right.
\end{aligned}
$$

where [...] is the first bracket in Eq. (151) and

$$
\omega_{R}^{T}=\left(\pi \Delta_{o} / 4 T\right)\left(2 \Gamma / \tau_{E}+\left(2 \Delta_{o}\right)^{2}\right)^{1 / 2}
$$

In the $q=0$ limit, $\omega_{+}$reduces to the longitudinal mode eigenfrequency while $\omega_{\text {- }}$ is connected with the transverse mode. For $\Delta_{0}>\Gamma$, Eq. (151) can be expanded for all $\mathrm{Dk}^{2}$ and arbitrary supercurrents:

$$
\begin{gathered}
\omega_{+}=\frac{-i}{2 \Delta_{0} \tau_{E}}\left(16 \mathrm{~T} B \Delta_{0}^{2} / \pi+\mathrm{Dk}^{2}-4 \mathrm{Dq}^{2}\right), \\
\omega_{-}=-i\left(\pi \Delta_{0}^{2} / 2 \mathrm{~T}\right)\left(1+\mathrm{Dk}^{2} / \omega_{\mathrm{R}}^{\mathrm{T}}\right) .
\end{gathered}
$$

At $q=q_{c}, 4 D q^{2}=16 T B \Delta_{0}^{2} / \pi$. Therefore $\left|\omega_{+}\right|$decreases to $\mathrm{Dk}^{2} /\left(2 \Delta_{0} \tau_{\mathrm{E}}\right)$ as $\mathrm{q}$ increases to $\mathrm{q}_{\mathrm{c}}$.

This limit is difficult to reach in $A l$ since $1 / \tau_{E} \simeq 0.1 \mu \mathrm{eV}$. The inelastic electron-phonui scattering rate is much higher in other superconductors such as $\mathrm{Pb}$, resulting in greater ease of observing such a mode softening. 
F. RELATED THEORETICAL WORK

1. Transverse Mode Calculations

Several authors have shown that the transverse mode dispersion relation is of the form

$$
i \omega=\omega_{D} \pm\left(\omega_{D}^{2}-c^{2} k^{2}\right)^{1 / 2}
$$

where $\omega_{D}$ is the damping rate and $c$ is the velocity of the mode. We have already indicated in part B of this chapter that EntinWohlman and Orbach reproduced Schmid and Schön's ${ }^{16}$ (SS) original calculation for dirty superconductors in the limit $\dot{\Gamma} \ll \omega \ll \Delta \ll T$. Recall from Eqs. (35) and (36) that

$$
\omega_{D}=\pi \Delta^{2} / 4 \mathrm{~T}
$$

and

$$
c=(2 \Delta D)^{1 / 2} \text {. }
$$

At about the same time Artemenko and Volkov ${ }^{43}$ (AV) calculated the clean limit $\left(V_{F} / \ell \ll \Delta\right)$ result. They found for the dispersion relation

$$
i \omega=i k c+\omega_{D}
$$

where

$$
c=\left(7 \zeta(3) \Delta / 3 \pi^{3} \mathrm{~T}\right)^{1 / 2}
$$

and

$$
\omega_{D}=\left(V_{F} / \ell\right)\left(2 \varepsilon+(k \ell)^{2} / 12\right)
$$

The effects of spin-flip scattering due to paramagnetic impurities on the clean limit results were calculated by Galaiko, 
Glushchik and Shumeiko ${ }^{44}$ (GGS). They found a dispersion relation which reduced to the $A V$ result for negligible spin-slip scattering $\left(\tau_{S} \rightarrow \infty\right)$. The scattering rate becomes important for $l / V_{F} \tau_{S} \geqslant\left(\Delta / T_{C}\right)^{4}$. In this concentration range shortwave damping is renormalized while the mode velocity is essentially. unaffected. As the scattering rate is increased to $l / V_{F} \tau_{S} \sim\left(\Delta / T_{C}\right)^{2}$ paramagnetic damping dominates and for $\ell / V_{F} \tau_{S} \sim \Delta / T_{C}$ it completely suppresses propagation.

Cheishvili ${ }^{45}$ calculated the effect of a large electron-phonon scattering rate $\left(\Delta^{2} / T_{c} \ll 1 / \tau_{E} \ll \Delta\right)$. He found the ss dispersion and velocity given in Eqs. (155) and (157) respectively. The damping, however, was increased by $1 / 2 \tau_{E}$ over the SS result (Eq. (156)).

$$
\omega_{D}=\pi \Delta^{2} / 4 T+1 / 2 \tau_{E}
$$

General expressions that are valid for any normal electron meanfree-path have been calculated by several authors. Using a two-fluid model Pethick and Smith ${ }^{46}$. (PS) found a dispersion relation similar to that of SS by calculating quasiparticle diffusion near phaseslip centers. The mode velocity and damping are

$$
c=\left(\mathrm{N}_{\mathrm{S}} / \mathrm{m} \mathrm{X}_{\mathrm{S}}{ }^{\mathrm{o}}\right)^{1 / 2}
$$

and

$$
\omega_{D}=N_{S}(1-\beta) / 2 \tau_{i m p} N
$$

Here $\mathrm{N}_{\mathrm{S}}$ is the superconducting electron density and $\mathrm{N}$ is the total density. $x_{S}{ }^{0}$ is defined as the susceptibility of the superfluid component in the absence of Fermi liquid effects. The quantities $\mathrm{N}_{S}$ and $\beta$ must be determined from microscopic calculations. Kadin, 
Smith and Skocpol ${ }^{47}$ (KSS) do a similar calculation for the propagating mode and show an analogy to propagation of electric signals down transmission lines.

Finally, Holstein, Kulik and $\operatorname{Orbach}^{48}$ (HKO) do a linear response theory based solely on the self-consistent BCS equation. They show that the Carlson-Goldman mode is obtained from the AndersonBogoliubov mode by including the Coulomb interaction. The SS and AV limits are again reproduced. Ihe low temperacure ( $\because \Delta)$ Iimil as well as the high temperature limit $(\Delta \ll \mathrm{T}$ ) is treated for both clean and dirty superconductors.

For $w \ll \Delta \ll T$ the SS dispersion relation (Eq. (155)) is obtained with a modified damping and velocity given by

$$
\omega_{D}=\left(\pi \Delta^{2} / 4 T\right) \times(2 \pi \tau T)
$$

and

$$
c=\left(2 \Delta \mathrm{D}_{X}(? \pi \tau \mathrm{T})\right)^{1 / 2}
$$

where $\tau=\ell / V_{F}$ and $X(x)$ is the Gor'kov function. The limiting values of the Gor'kov function are

$$
x(2 \pi \tau T)= \begin{cases}1 & 2 \pi \tau T \ll 1 \\ 7 \zeta(3) / 2 \pi^{3} \tau T & 2 \pi \tau T \gg 1\end{cases}
$$

From Eqs. (164)-(166) one can reproduce the SS and AV results.

A high-frequency $(\omega \sim \Delta)$ dispersion relation is also calculated. It is found that

$$
i \omega=\gamma(k)+i \omega(k)
$$


where

$$
\omega(k)=\left[-\Omega^{2} / 2+(\Omega / 2)\left(\Omega^{2}+16 \Delta^{2}\right)^{1 / 2}\right]^{1 / 2} .
$$

The quantity $\Omega=\mathrm{Dk}^{2} \mathrm{X}(2 \pi \tau \mathrm{T})$ and

$$
\gamma(k)=2 \omega_{D}\left(1-\omega^{2}(k) / 4 \Delta^{2}\right) /\left(2-\omega^{2}(k) / 4 \Delta^{2}\right)
$$

In this high-frequency limit the damping vanishes at $\mathrm{Dk}^{2} \gg \Delta$, while the resonant frequency saturates at $2 \Delta$.

The Schmid Mode

Schmid $^{49}$ and $\mathrm{HKO}^{48}$ calculate a resonant frequency at $\omega=2 \Delta$ for the $k=0$ longitudinal mode, that is for fluctuations in the order parameter modulus. The physical origin of this resonance is that when the order parameter is perturbed it tries to relax. But if a pair of quasiparticles attempt to relax into a Cooper pair, they must gain energy $2 \Delta$ violating energy conservation. According to quantum mechanics this violation is allowed for a time $t \leq h / 2 \Delta$. This allows the order parameter to oscillate at a frequency $\omega \geq 2 \Delta / \hbar$. In the absence of scattering, this oscillation would decay as $t^{-1 / 2}$. The presence of scattering results in the more conventional exponential decay. ${ }^{48}$ In light of this, the mode calculated from the Schön and Ambegaokar dispersion relation (see Eq. (125b)) Is probably the Schmid mode.

\section{Schmid and Schön Relaxation Times}

Schmid and Schön ${ }^{15}$ calculated the branch imbalance of charge relaxation time $\tau_{Q}$ and the gap relaxation time $\tau_{\Delta}$. These times are 
not contained in the pair-field susceptibility of the orderparameter and therefore are unrelated to the data reported here. Their calculation, however, is intimately related to the calculation of order-parameter relaxation times. A brief summary will be given here for the sake of completeness.

Schmid and Schön found

$$
\tau_{Q}=(4 \mathrm{~T} / \pi \Delta) \tau_{E}
$$

and

$$
\tau_{\Delta}=\left(\pi^{2} \mathrm{~T} / 7 \zeta(3) \Delta\right) \tau_{F} .
$$

Pethick and Smith ${ }^{46}$ determined that for $\tau_{Q}$ one must average $\tau_{E}{ }^{-1}$ over the Fermi surface while for $\tau_{\Delta}$ it is $\tau_{E}$ itself that must be averaged.

Lawrence and Meador ${ }^{50}$ calculate the above lifetimes for aluminum for temperatures down to absolute zero. The result is uscd to calculate the order-parameter relaxation times just helow $\mathrm{T}_{\mathrm{c}}$. 


\section{EXPERIMENTAL TECHNIQUES}

In the first part of this chapter a detailed description of the technique used for fabricating $\mathrm{Al}_{1-\mathrm{x}} \mathrm{Er} \mathrm{x}-\mathrm{Al}_{2} \mathrm{O}_{3}-\mathrm{Pb}$ junctions will be given. Details pertaining to the sample geometry are given by Carlson and Goldman. ${ }^{3,4}$ Discussion of $\mathrm{Pb} / \mathrm{Ag}$ - $\mathrm{Pb}$ proximity sandwich junctions will be given in Appendix II as it is unrelated to the main body of this dissertation.

Section B of this chapter is devoted to a detailed description of the cryostat that was built for the experiments reported here.

Finally, section $C$ contains a description of the data acquisition hardware. Most of the equipment was used by Carlson ${ }^{3}$ and some of it by Anderson. 8 Unnecessary duplication in the description of the hardware will be avoided. Details not reported here can be found in their respective dissertations. 


\section{A. FABRICATION OF $\mathrm{Al}_{1-\mathrm{x}} \mathrm{Er}_{\mathrm{x}}-\mathrm{Al}_{2} \mathrm{O}_{3}-\mathrm{Pb}$ JOSEPHSON JUNCTIONS}

1. The Evaporation System

All of the tunneling junctions used in the experiments reported here were fabricated in an oil-free high vacuum system. Rough pumping was accomplished in two stages. First the evaporation chamber was pumped to about 50 Torr using a dry vane pump. Two Ultek molecular seive sorption pumps completed the roughing step to a pressure less than $10^{-2}$ Torr. At this point the system was baked for two to eight hours depending on the relative humidity and the length of time the system was exposed to room air. Baking was accomplished by utilizing three heat sources. Infrared heat-lamps were directed at the upper portion of the evaporation chamber through the glass bell jar. A resistive heater capable of dissipating 2000 watts was clamped to the lower portion of the chamber. Optimum dissipation was found to be 500 watts. The third source of heat was a spent titanium filament. By operating a used filament at 30 amps, the interior of the system, which included copper tubing used as a water cooled trap during high vacuum pumping, could readily be outgassed. After this bake-out the titanium filaments to be used during evaporation were outgassed and the sorption pumps were 1solated from the chamber. The system was now ready for pumping to high vacuum.

Separated from the evaporation chamber by a six-inch gate-valve is a 350 liter per second Ultek ion pump. The ion pump chamber maintained a pressure of $10^{-8}$ Torr when not exposed to evaporation 
chamber. Afțer the above roughing and bake-out procedure the gatevalve was opened. Along with titanium getter pumping aided by water and air cooling, the ion pump was capable of pumping the system to the low $10^{-8}$ scale in a short time. 
2. Preparation of the $\mathrm{Al}_{1-\mathrm{x}} \mathrm{Er}_{\mathrm{x}}$ Alloy

The first step in making the $\mathrm{Al}_{1-\mathrm{x}} \mathrm{Er}_{\mathrm{x}}-\mathrm{Pb}$ junction was to prepare a source for the alloy electrode. The procedure used is a modification of the method of Craven, et al. ${ }^{36}$ A $5 \mathrm{~N}$ pure Al foil ${ }^{51}(0.0127 \mathrm{~cm}$ thick) was loaded into the vacuum system. Erbium of $3 \mathrm{~N}$ purity was evaporated onto the Al foil to a thickness of about $10^{-4} \mathrm{~cm}$. To prevent oxidation of the Er, $200 \AA$ of pure Al was evaporated on top of the Er. The Er concentration in this master charge was determined by carefully weighing the bare Al foil and the Al/Er sandwich. To prepare a sample charge of desired concentration enough pure Al foil was folded together with a portion of the master charge to dilute the Er. Only enough material was used to provide a $1000 \AA$ film when evaporated to completion. In contrast to the Craven method we did not cold roll the charge. Doing so would only add additional impurities as well as oxidize a portion of the Er. At the melting point of $\mathrm{Al}$, Er is soluable to about one atomic percent. 52 Since our entire charge was evaporated in one shot, premixing would have served no purpose. 
3. Fabricating the Junction

Once the necessary materials were loaded into the evaporator and the system was pumped to high vacuum, actual fabrication of the junction could begin. The first step was to flash evaporate the Al/Er charge to completion from a tungsten filament at a rate of $200 \AA / \mathrm{sec}$. This alloy was then coated with $50 \AA$ of pure Al which was evaporated from a tungsten filament at a rate of about $10 \AA / \mathrm{sec}$. This prevented oxidation of the $\mathrm{Er}$ and also provided a reproducible surface for forming the oxide barrier.

Oxidation of the alloy electrode is the most critical step in the process. With the ion pump isolated from the evaporator, dry oxygen was admitted to the evaporation chamber to a pressure in the range $5 \times 10^{-3}$ to $5 \times 10^{-2}$ Torr for a period of 1 to 30 minutes. The parameters for best results would vary over a period of months but would usually be stable for several days or even weeks. Thus two or three trials were necessary to obtain a junction of suitable resistance if several weeks had passed since the previous fabrication.

Following the evacuation of the oxygen gas from the evaporation chamber the edges of the electrodes were masked with $1000 \AA$ of $\mathrm{Bi}_{2} \mathrm{O}_{3}$. The $\mathrm{Bi}_{2} \mathrm{O}_{3}$ was evaporated from a platinum boat and deposited at a rate of $100 \AA \AA / m i n$. The junction was completed by evaporating $\mathrm{Pb}$ as the counterelectrode. $2000 \AA$ of $5 \mathrm{~N} \mathrm{~Pb}$ was deposited at a rate of $100 \AA / \mathrm{sec}$. Additional leads and pads for electrical contact were deposited to complete the device. Although one junction survived at room temperature for more than six hours without 
exhibiting substantial leakage at temperatures near $1 \mathrm{~K}$, most

junctions were kept at room temperature for less than one hour. 


\section{B. CRYOSTAT DESIGN}

The cryostat designed for these experiments was constructed primarily from non-magnetic stainless steel and OFHC copper. To minimize the possibility of leaks due to corrosion from solder flux most of the permanent joints were made by welding. The temporary joints were made with a cryogenic epoxy. 53 .

A brief outline of the cryostat is as follows: From the top plate of $3 / 8 "$ stalnless steel dre suspended the thin-wall stainless steel tubes for pumping, electrical feed-throughs, etc. which in turn are attached to the bottom plate of OFHC copper: Three radiation plates of OFHC copper are epoxied to the stainless steel tubes at random intervals: These plates facilitate heat exchange between the helium gas and the cryostat and also provide structural support in addition to blocking the room temperature radiation from the top plate of the cryostat. The vacuum can is attached to the bottom plate and sealed with an indium 0-ring. Suspended within the vacuum can is the $1 \mathrm{~K}$ pot, that is an isolated chamber capable of being filled with liquid he and pumped to the vapor pressure of liquid He at $1 \mathrm{~K}$. Attached to the $1 \mathrm{~K}$ pot is the exchange gas can which is also sealed with an indium 0-ring. The purpose of this can is to provide the capability of improving the thermal contact between the sample block and the $1 \mathrm{~K}$ pot. The sample block is attached to the $1 \mathrm{~K}$ pot with nylon screws and is thermally isolated with $3 / 8^{\prime \prime}$ delrin spacers. A controlled thermal link between the $1 \mathrm{~K}$ pot and the sample block is accomplished by the use of a 4" long by .03" thick 
copper wire. A schematic of the low temperature end of the cryostat is given in Fig. (2).

The electrical leads for the sample area were fed through the exchange gas pumping line. Heat-sinking to the $4 \mathrm{~K}$ bath was accomplished by wrapping the wires around a copper-heat sink which was exposed to the $4 \mathrm{~K}$ bath. The copper heat-sink was sealed to the stainless steel tubes with cryogenic epoxy. This epoxy seal has been cycled dozens of times from $300 \mathrm{~K}$ to $4 \mathrm{~K}$ without failure. Even quenching from $300 \mathrm{~K}$ to $77 \mathrm{~K}$ did not cause failure. The seal was susceptible to failure only through mechanical stress such as that encountered when the $1 \mathrm{~K}$ pot fill valve was severely torqued. Once this was realized, failure of the seal was extremely rare.

The $1 \mathrm{~K}$ pot fill valve was fashioned from a right angle needle valve. In order to facilitate a slow refill of the $1 \mathrm{~K}$ pot while cold, a stainless steel ball was inserted between the valve seat and needle. This sealed very well and allowed refilling of the $1 \mathrm{~K}$ pot without substantial warming of the sample block.

The sample block contained eight spring-loaded copper contacts electrically isolated with delrin sleeves. The base of the sample block was threaded and served as a heat-sink for the electrical leads and the $100 \mathrm{ohm}$ manganin wire heater. All wires were plotted with G.E. \#7031 varnish.

A total of fifteen twisted pairs of wires entered the sample area. Two pairs were used for each of three temperature sensors, four pairs for electrical measurements on the tunneling junction, 


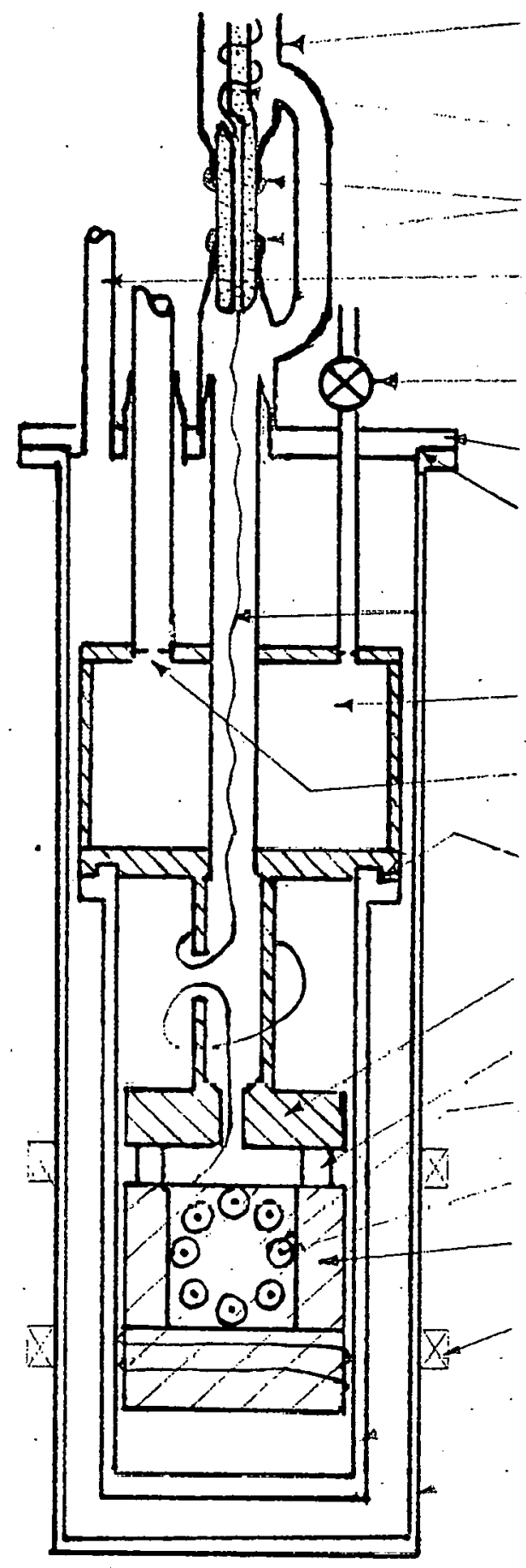

PUMPING LINE AND ELECTRICAL

FEED-THRU FOR SAMPLE AREA

COPPER HEAT-SINK

EPOXY SEAL

VACUUM CAN PUMP LINE

1K POT FILL VALVE

BOTTOM PLATE

INDIUM O-RING SEAL

ELECIRICAI LEAUS

IK POT RESERVOIR

IK POT PUMP LINE

INDIUM O-RING SEAL

1K POT HEAT-SINK AND MOUNTING PLATFORM

INSULATING SPACERS

INSULATING SLEEVE

SPRING-LOADED COPPER CONTACTS

SAMPLE BLOCK

HELMHOLTZ COIL SUPERCONDUCTING MAGNET

EXCHANGE GAS CAN

VACUUM CAN

Figure 2. Schematic of the low-temperature part of the cryostat. 
one pair for the sample block heater and four pairs for spares in case of broken wires as the cryostat aged.

At the top of the cryostat the wires destined for the sample area were of copper and were connected to low pass pi filters in the cable connector. The copper wires were soldered to superconducting NbTi wires clad in a copper-nickel matrix ${ }^{54}$ at the $4 \mathrm{~K}$ bath heat sink. This arrangement allowed the lowest resistance while minimizing the heat leak to the $1 \mathrm{~K}$ pot. The wires were also heat-sunk to the $1 \mathrm{~K}$ pot and the sample block.

A superconducting Helmholtz magnet that could be operated in the persistent mode was mounted on the vacuum can. The magnet produced 172 Oe per amp and was capable of more than 3000 De at the sample. Calculation of the magnet calibration from the magnet geometry agreed to within $0.3 \%$ of the calibration determined independently by using a pickup coil in a time-varying field. 
C. DATA ACQUISITION

A schematic of the data acquisition system is shown in Fig. (3). In order to measure the current-voltage characteristics over a wider range of temperature and magnetic field (in particular at temperatures and magnetic fields where the tunneling characteristics exhibited negative dynamic resistance) a shunt resistor was placed across the current leads to the junction. A resistor in series with the junction allowed accurate measurements of the current actually going through the junction. A chopped dc current was used in order to take advantage of ac voltage measuring techniques while not introducing errors that might result from the non-1inear nature of the current-voltage characteristics.

Details pertaining to the ramp generator, chopper, computer facilities and thermometry have been given elsewhere. ${ }^{3,6}$

Before each current-voltage trace was taken, the magneric field was set to a predetermined value such that the zero voltage Josephson current was at a minimum. The temperature was set and stabilized to $\pm 2 \times 10^{-5} \mathrm{~K}$ by monitoring the resistance of a germanium resistor ${ }^{53}$ which had previously been calibrated as a function of temperature. Each current-voltage characteristic consisted of $250^{\circ}$ daca poincs. Each dala pulul was lie diverage of about 100 readings of the analog-to-digital converters.

The data were plotted on the display terminal screen for immediate analysis. Based on the analysts lie dald wele either discarded or stored on magnetic disk for future use. For more sophisticated fitting than could be accomplished by the minicomputer 


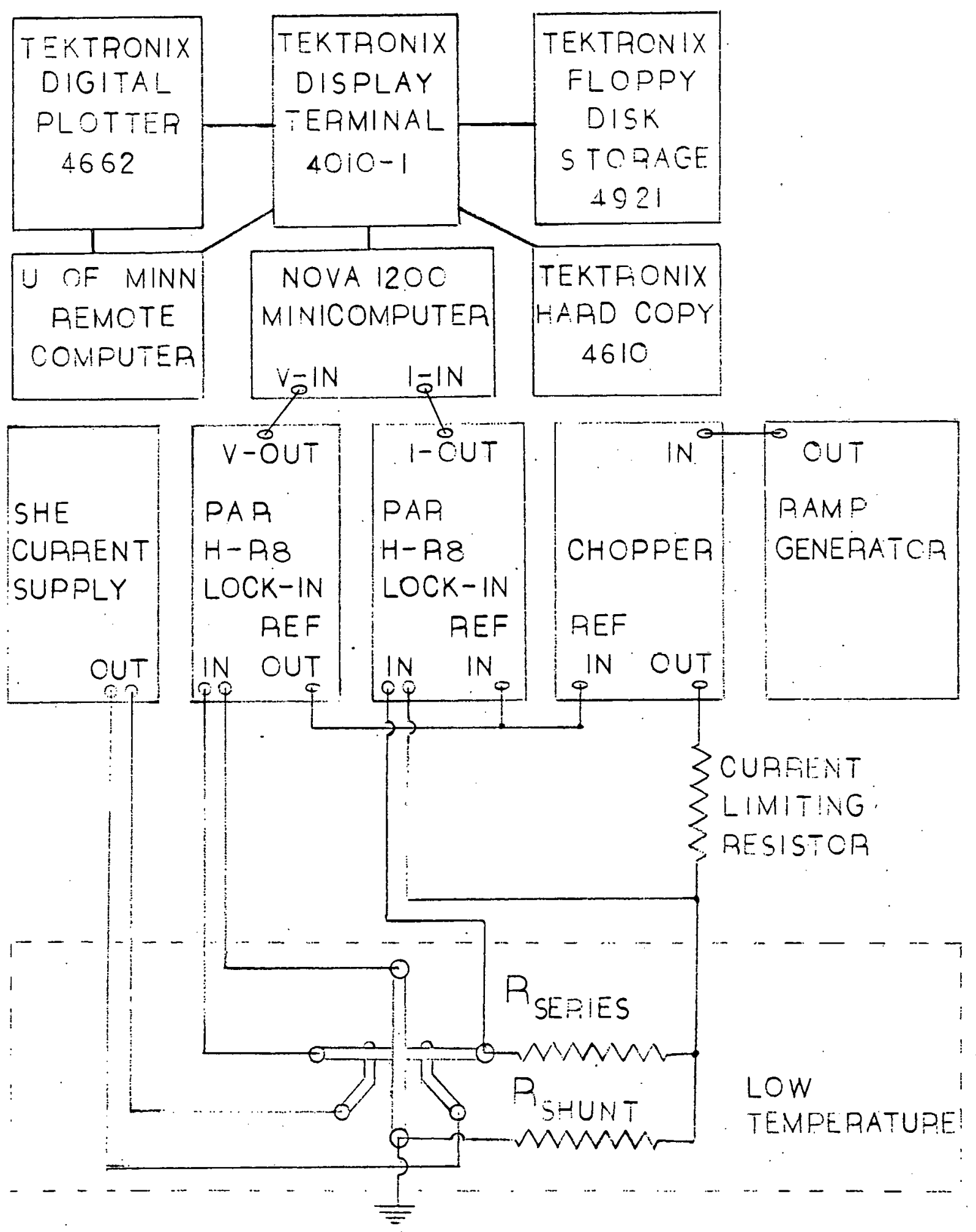

Figure 3. Block diagram of the data acquisition system. 
the data were transmitted to the University of Minnesota remote computers for further analysis. 


\section{ANALYSIS OF THE DATA}

There are basically three distinct phases of data analysis. A preparatory phase includes calibration of the various amplifiers and sensors and measurement of the normal state tunneling resistance. The second phase is on-line analysis of tunneling data. From the data at temperatures just above $\mathrm{T}_{c}$ of the alloy electrode we determine the temperature and magnetic field dependent relaxation frequency $\omega_{R}(T, H)$. At the same time we determine the $\mathrm{Pb}$ gap, $\Delta_{\mathrm{Pb}}$ and leakage conductance, $G_{L}$ which, along with $T$ and $R_{N}$, determine the background current for $T<T_{C}$. The diffusion constant $D$ is determined from the magnetic field dependence of $\omega_{R}(T, H)$.

In the third phase we fit the data using a remote computer. From the data for $\mathrm{T}>\mathrm{T}_{\mathrm{C}}$ we determine the pair-breaking parameter. The excess current for $T<T_{c}$ is fit to a function containing three peaks. Five of the parameters in the fit can be Identified as the real or imaginary part of a characteristic frequency and can be compared with the various theoretical predictions.

The remainder of this chapter is devoted to a more detailed description of the analysis techniques. 
A. NORMAL STATE TUNNELING RESISTANCE

The normal state tunneling resistance is measured at a temperature of about $8 \mathrm{~K}$. The temperature dependence below this temperature is weak and can be ignored. The measurement is an ac four terminal measurement at $400 \mathrm{~Hz}$. Unless the condition $R_{N} \gg R_{\square A 1}+R_{\square P b}$ is met, where $R_{\square}$ is the sheet resistance, one must make corrections due to the geometry. For the devices studied here, $R_{\square A l} \simeq 0.1$ ohms and $R_{\square P b} \leqslant 0.001$ while $R_{N} \geq 0.1$ ohms. Thus the measured $\mathrm{R}_{\mathrm{N}}$ must in general be corrected for finite sheet resistance.

The temperatures were determined by measuring the resistance of a precalibrated germanium resistor. ${ }^{55}$. Absolute accuracy was $\pm 5 \times 10^{-3}$ K. Temperature differences were accurate to $\pm 0.5 \%$. The temperature could be stabilized to $\pm 2 \times 10^{-5} \mathrm{~K}$. 
B. QUASIPARTICLE TUNNELING AND LEAKAGE CONDUCTANCE

At temperatures $\mathrm{T}_{c}(\mathrm{Al})<\mathrm{T}<\mathrm{T}_{\mathrm{c}}{ }^{\prime}(\mathrm{Pb})$ there are three contributions to the measured current for voltages less than the $\mathrm{Pb}$ gap. The total current $I_{T}$ is

$$
I_{T}=I_{q p}+G_{L} V+I_{e x}
$$

Here $I_{q p}$ is the quasiparticle tunneling and $G_{L}$ is the leakage conductance. In this temperature range the functional form of $I_{e x}$ is the well-known quasi-Lorentzian.

$$
I_{e x}=2 I_{P}\left(V / V_{P}\right) /\left(I+\left(V / V_{P}\right)^{2}\right)
$$

The single particle tunneling current can be calculated exactly if $\mathrm{R}_{\mathrm{N}}, \Delta_{\mathrm{Pb}}$ and $\mathrm{T}$ are known. 56

$$
1_{\mathrm{qp}}=2 \frac{\Delta_{\mathrm{Pb}}}{\mathrm{R}_{\mathrm{N}}} \sum_{1}^{\infty}(-1)^{\mathrm{m}+1} \overline{\mathrm{K}}_{1}\left(\mathrm{~m} \frac{\Delta}{\mathrm{T}}\right) \sinh \left(\mathrm{m} \frac{\mathrm{v}}{\mathrm{T}}\right)
$$

Here $\mathrm{K}_{1}$ is the modified Bessel function of the second kind.

The excess current is significant even in the range $\mathrm{V}>200 \mu \mathrm{V}$.

For this reason the parameters $I_{P}, V_{P}, \Delta_{P b}$ and $G_{L}$ were varied simultaneously to achieve the best fit of $I_{T}$ to the measured current.

In Flg. (.4a) we have plotted a typical current-voltage characteristics along with the fitted contributions from $I_{q p}$ and $G_{L}$ V. Fig. (4b) is a plot of the current less the background along with theoretical quasi-Lorentzian Lineshape.

The best fit is sensitive to variations in $\Delta_{\mathrm{Pb}}$ of about $1 \mu \mathrm{V}$. At. $\mathrm{T} \simeq 1.2 \mathrm{~K}$ it is found $\Delta_{\mathrm{Pb}} \simeq 1.36 \mathrm{mV}$. If a small $(\sim 1 \%)$ adjustment is 


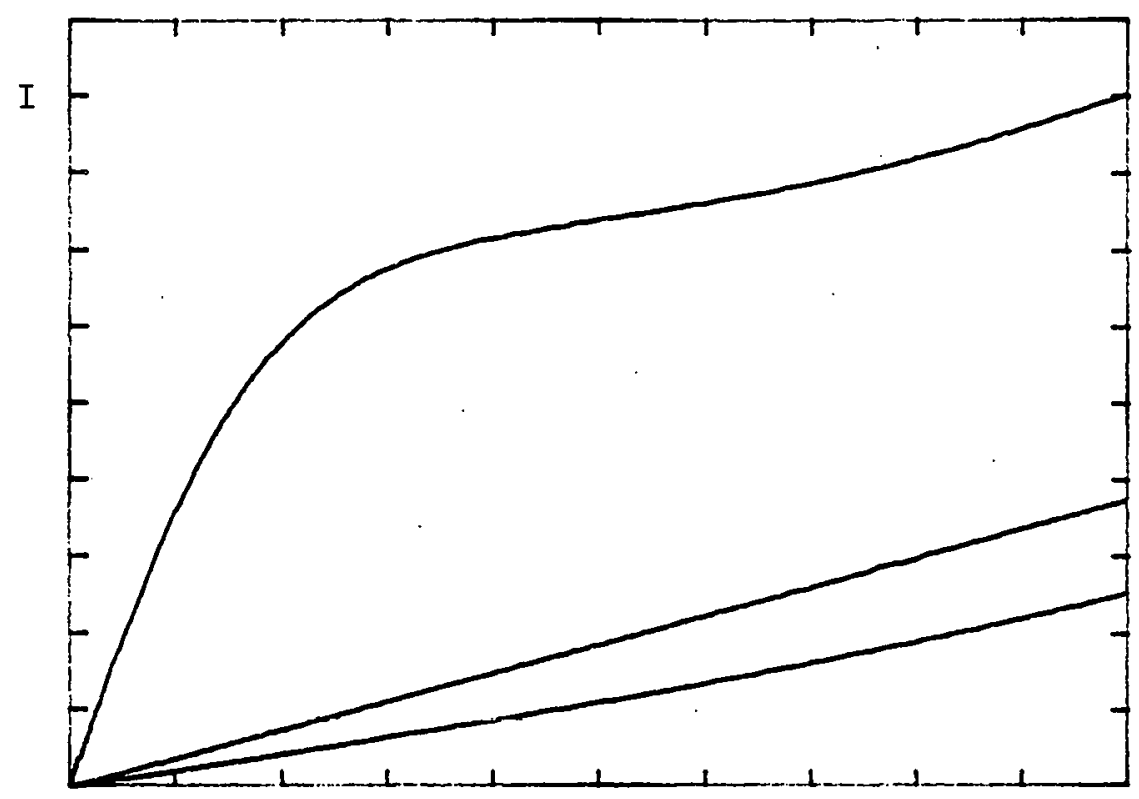

(a)

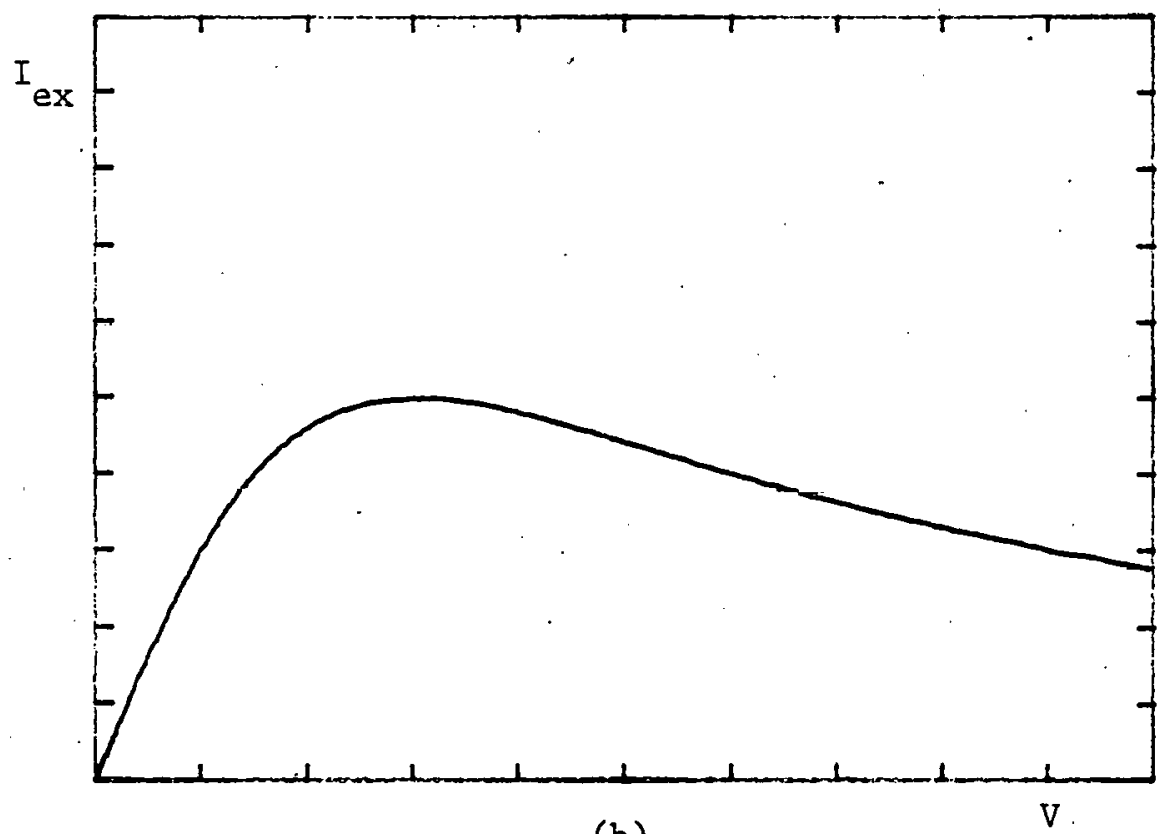

(b)

Figure 4. (a) Typical total current, leakage current, and quasiparticle current vs: voltage from top to bottom respectively.

(b) Excess current obtained by substracting the leakage and quasiparticle currents from the total current in (a). 
made to the measured value $R_{N}$ one can find a single value for $G_{I}$ and for $\Delta_{\mathrm{Pb}}$ that will give an excellent fix for $I_{T}$ to the measured current for $T_{C}<T \leqslant T_{C}+100 m K$. Thus a set of $R_{N}, G_{L}$ and $\Delta_{P b}$ can be determined at temperatures greater than $T_{C}$ and used for temperatures less than $T_{c}$ where the exact functional forms for $I_{e x}$ are not known.

It should be noted that small charges in $R_{N}{ } G_{L}$ and $\Lambda_{\mu b}$ तn not have a measurable effect on the best value for $V_{p}$. This is important when the value of $\mathrm{V}_{\mathrm{P}}(T)$ is used to determine the pairbreaking parameter. $V_{P}$ can be measured to instrumental accuracy. $(\sim .05 \mu \mathrm{V})$ at temperatures near $T_{C}$ where the excess current dominates. 


\section{PAIR TUNNELING AND EIGENFREQUENCIES}

It was indicated in the last section that there is only one eigenfrequency above $T_{c}$, and the determination of that frequency was a natural consequence of determining the background current.

At temperatures below $\mathrm{T}_{c}$ we are less fortunate in that exact functional forms are not known for the excess current. The experimental features are shown in Fig. (5). It is believed that the low voltage (frequency) shoulder (or peak) is due to the diffusive longitudinal mode. The mid-frequency peak is described as a propagating mode (the eigenfrequency contains a real and imaginary part with the real part linear in $k$ (magnetic field)). The high frequency step (or peak) occurs near the gap voltage and may also be due to the longitudinal mode.

In order to describe this with a general function, eight parameters are needed: three amplitudes, location of low frequency peak, location and width of mid-frequency peak, and location and sharpness (width) of the high frequency peak. A function that containe all of these featuree ie

$$
\begin{aligned}
x^{\prime \prime}(\omega)= & \frac{2 A_{L} \omega / \omega_{R}^{L}}{1+\left(\omega / \omega_{R}^{L}\right)^{2}}+\frac{A_{T} \omega_{D} \omega}{\omega_{D}^{2}+\left(\omega-\omega_{R e s}\right)^{2}} \\
& +\frac{A_{T} \omega_{D} \omega}{\omega_{D}^{2}+\left(\omega+\omega_{\text {Res }}\right)^{2}}+\frac{A_{G}}{1+\left(\omega_{G} / \omega\right) P}
\end{aligned}
$$

The first term is a quasi-Lorentzian which is peaked at the relaxation frequency $\omega_{R}$ : The second and third terms describe the 


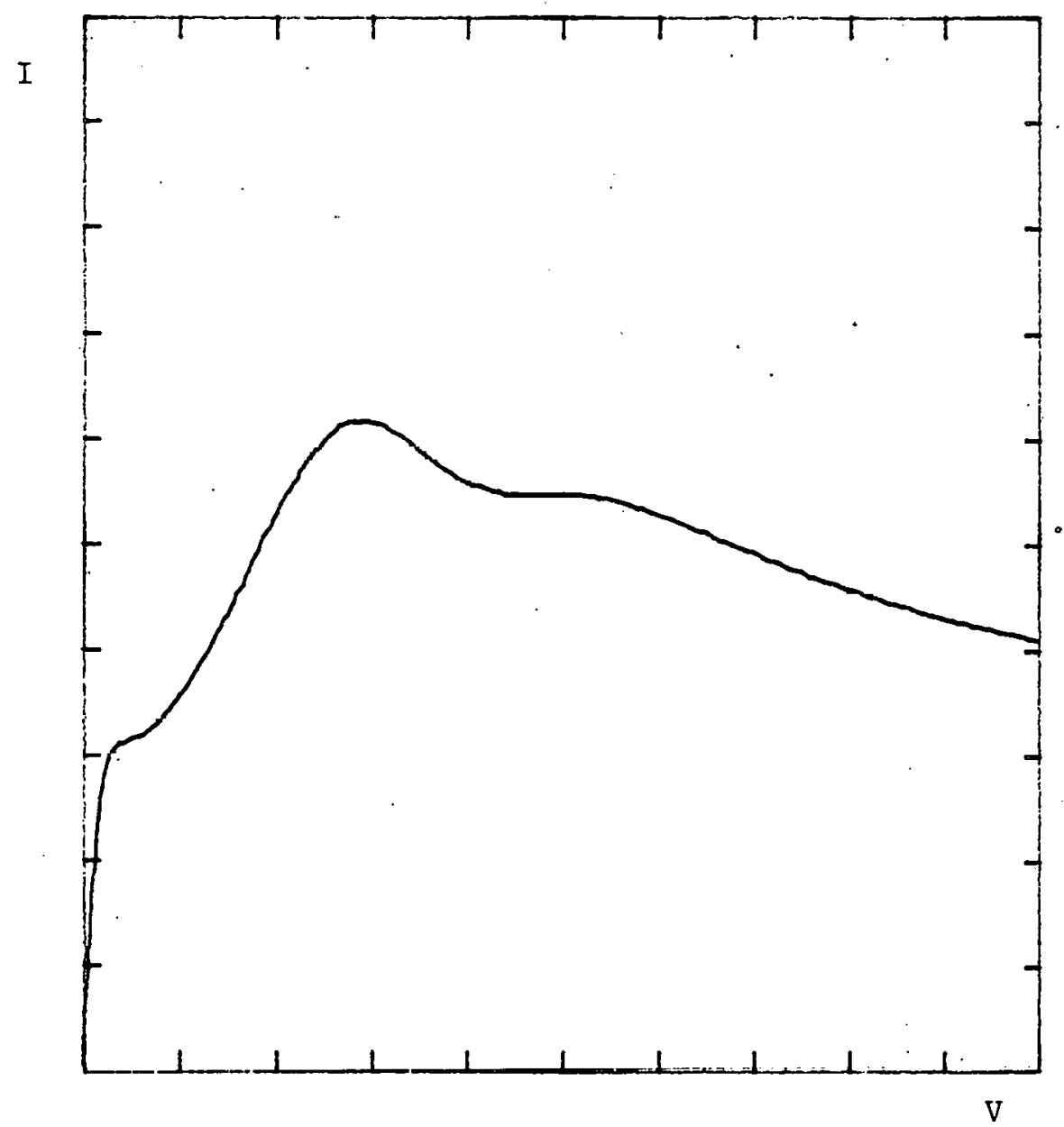

Figure 5. Typical excess current for $T<T_{c}$. 
propagating mode with resonant frequency $\omega_{\text {Res }}$ and damping $\omega_{\mathrm{D}}$ corresponding to an eigenfrequency of

$$
i \omega=\omega_{D}+i \omega_{\operatorname{Res}}
$$

Two terms are used for the transverse mode to make the current antisymmetric.

The last term in Eq. (175) represents a rounded step at the frequency $\omega_{G}$. The width of the step is $\sim 2 \omega_{G} / p$.

In order to determine the eigenfrequencies of the gap mode one can consider the associated structure function:

$$
S_{G}(\omega) \propto \frac{1 / \omega}{1+\left(\omega_{G} / \omega\right)^{P}}
$$

The peak of this function can be regarded as the real part of the eigenfrequency. The peak occurs at

$$
\omega_{\text {Res }}^{G}=(P-1)^{1 / P} \omega_{G}
$$

If one defines the damping of this mode to be the lower half-width of the structure function, the imaginary part of the eigenfrequency $\omega_{D}^{G}$ is the solution of

$$
1+P-(1-P) \omega_{D}^{G} / \omega_{\text {Res }}^{G}=\left(1-\omega_{D}^{G} / \omega_{\text {Res }}^{G}\right)^{1-P} \text {. }
$$

It can be shown that for all values of the exponent $P$, the damping frequency $\omega_{D}^{G}$ is less than $\omega_{\text {Res }}^{G}$. Therefore the functional form will be invalid whenever $\omega_{D}^{G}>\omega_{\text {Res }}^{G}$.

Other functions have been used for fitting to the transverse mnde. 
The functional form does not significantly alter the numerical values for the eigenfrequencies. It should also be noted that altering any one parameter by a significant amount results in a fit in which the other seven parameters do not change by more than $6 \%$. The one exception to these claims is that near $T_{c}$ more than one solution can be found since the modes are not well resolved. We found that the expression given in Eq. (175) represents the data over largest temperature span and is adequate for all of the samples analyzed. The fitting scheme is a modified Levenberg-Marquardt algorithm. 57 By systematically varying each parameter we have determined the fitting parameters are in error by no more than $6 \%$ except for $T_{c}-T<0.005 \mathrm{~K}$. 


\section{RESULTS AND DISCUSSION}

In the first section of this chapter we present the measured relaxation frequencies for $T>T_{c}$ for six samples with differing amounts of magnetic impurities. By fitting $\omega_{R}(T)$ to the expression

$$
\ln \left(T / T_{c}\right)+\psi\left(1 / 2+\rho-\omega_{R} / 4 \pi T\right)-\psi\left(1 / 2+\rho_{c}\right)=0
$$

with $\rho_{c}$ and $T_{c}$ as adjustable parameters, one obtains the pairbreaking energy $\Gamma$, where

$$
\Gamma=2 \pi \rho_{c} T_{c}
$$

These values are compared to values calculated from the impurity concentration.

In section $B$ we present the data for $T<T_{c}$. Eigenfrequencies are obtained from an eight parameter fit to the data. These are compared to the calculated eigenfrequencies of the various theories. Eigenfrequency data taken with a superimposed transport current is compared to calculations based on the Schön-Ambegaokar theory.

Finally in Section $C$ we summarize the experiments of other workers which are related to the experiments reported here. 
A. DATA FOR $\mathrm{T}>\mathrm{T}_{\mathrm{C}}$.

1. Measurements of the Pair-breaking Parameter

As discussed in Section II-B the relaxation frequencies at temperatures greater than $T_{c}$ are solutions of:

$$
\ln \left(T / T_{c}\right)+\psi\left(1 / 2+\left(D k^{2}-i \omega\right) / 4 \pi T+\rho\right)-\psi\left(1 / 2+\rho_{c}\right)=0
$$

The approximate solution,

$$
i \omega=D k^{2}+(8 / \pi)\left(T-T_{c}\right) f(\rho)
$$

is not adequate to determine $p$ from data extending to $\varepsilon \geq 0.1$. Fig.

(6) is a plot of relaxation frequencies for four samples with differing values of $\rho$. All of the data were taken in the absence of a magnetic field. The data for samples $\# 54$ (0\% Er), \#62 $(0.31 \%$ Er), $\# 45(0.62 \% \mathrm{Er})$ and $\# 38(0.98 \% \mathrm{Er})$ are displayed from top to bottom. Note that the data for sample $\#_{54}$ do not coincide with the dashed line, which is a plot of $\omega_{R}=8\left(T-T_{C}\right) / \pi$. The deviation is due to higher order terms in $\varepsilon$ and is clear evidence that the first order solution represented by Eq. (18.3) is inadequate.

In Fig. (7) we plot $\pi \omega_{R} / 8\left(T-T_{c}\right)$ as a function of $T-T_{c}$. In this plot we can clearly see the effects of finite $\varepsilon$ as well as finite $\rho$. Each set of points is nearly a straight line intercepting the vertical axis at the value $f\left(\rho_{c}\right)$. The superimposed lines are a least squares fit of the data to Eq. (180) with $\rho_{c}$ and $T_{C}$ treated as adjustable parameters. The data shown are for samples $\# 54(0 \% \mathrm{Er}), \# 62(0.31 \% \mathrm{Er}), \# 45(0.62 \% \mathrm{Er})$ and $\# 38(0.98 \% \mathrm{Er})$. 


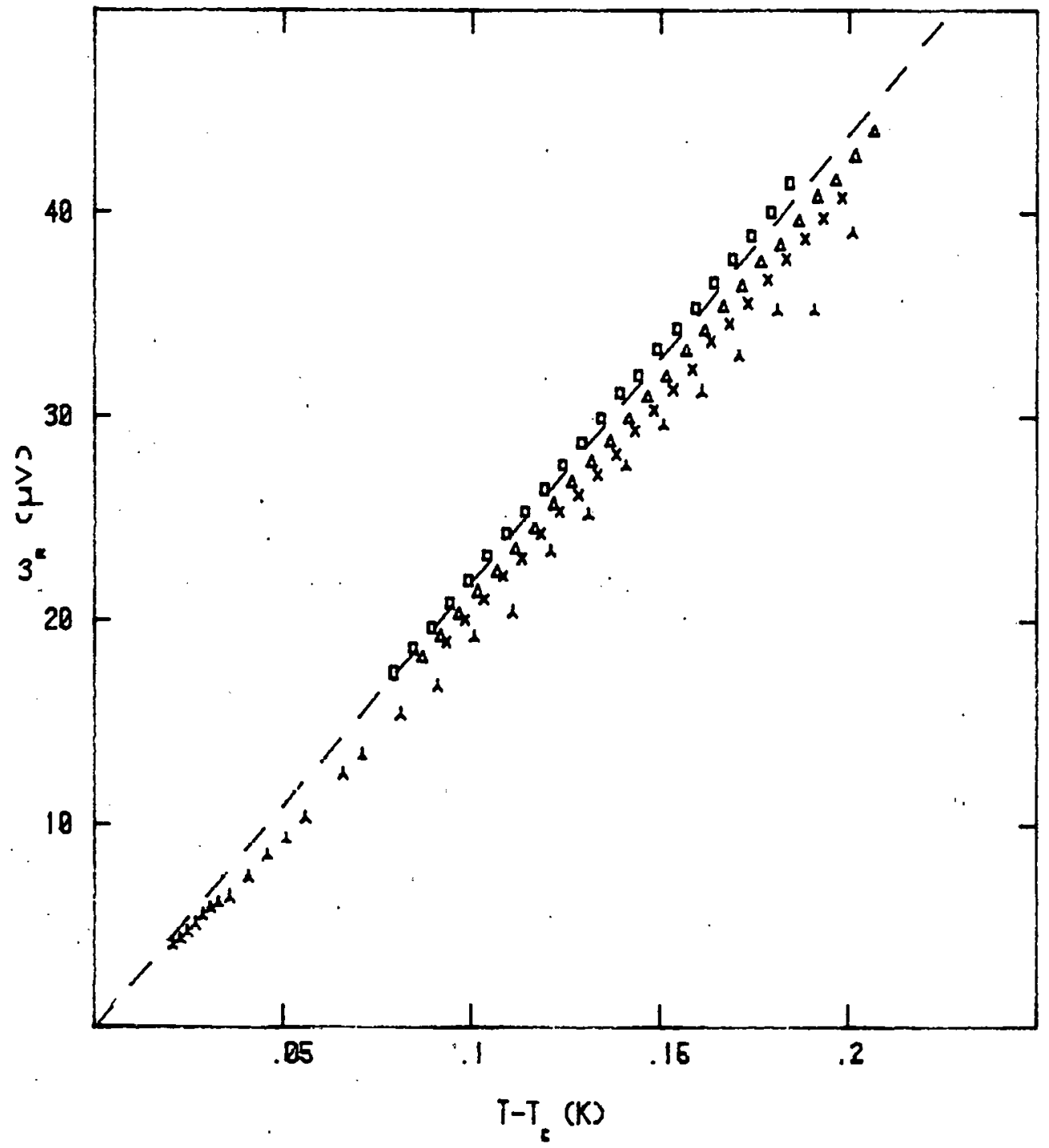

Figure 6. Relaxation frequencies for $T \geqslant I_{c}$ for samples \#54(a), \#62 $(\Delta)$, 非5 $(X)$, and \#38( $(\lambda)$. The dashed line is the asymptote for $\mathrm{T} \rightarrow \mathrm{T}_{c}$ for $\rho=0$. 


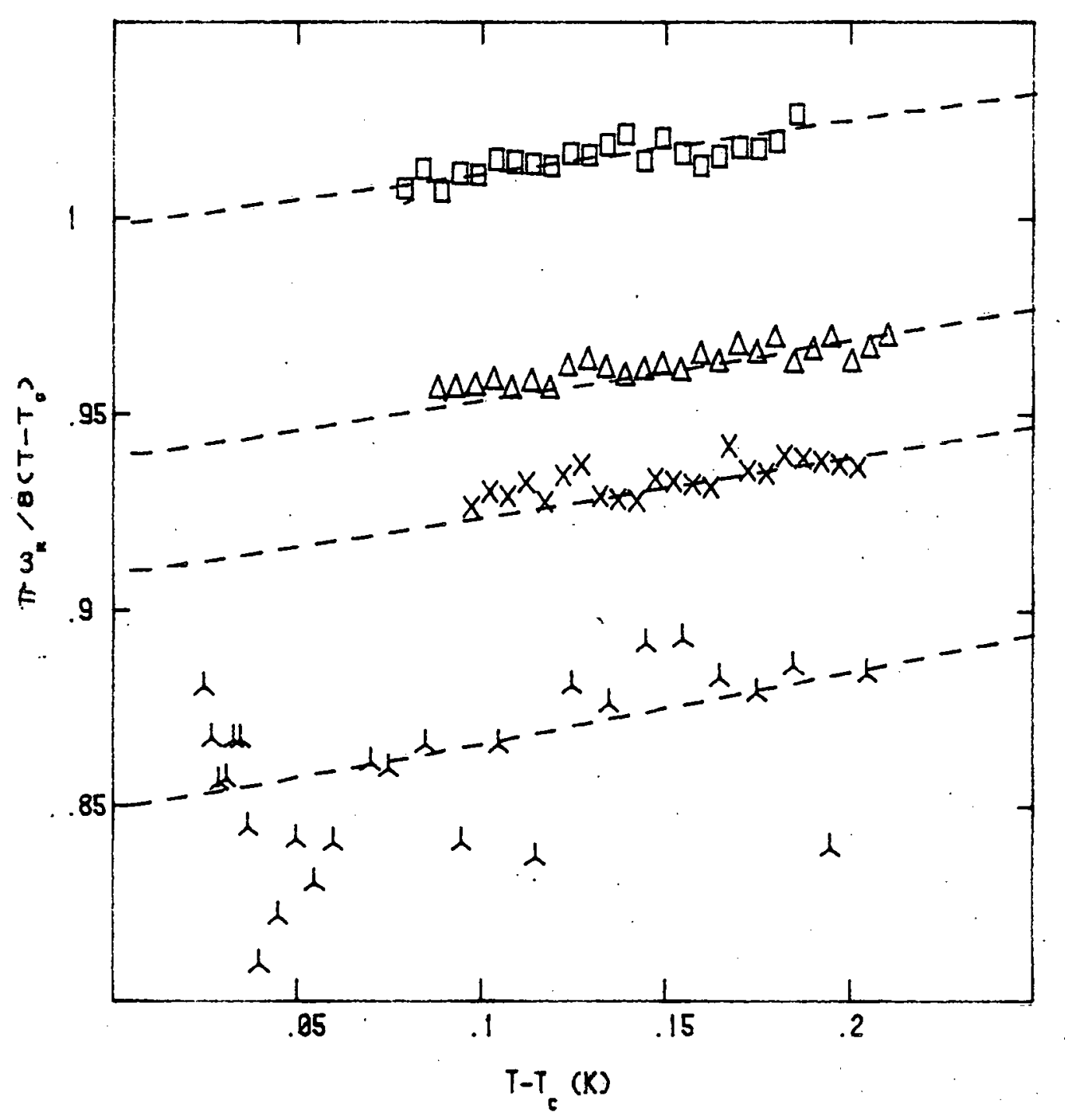

Figure 7. Relaxation frequencies normalized to $8\left(\mathrm{~T}-\mathrm{T}_{\mathrm{c}}\right) / \pi^{\prime}$ and best fit to $\rho_{c}$ and $T_{c}$.

(D) \#54, $\rho_{c}=0, T_{c}=1.221 \mathrm{~K} ;(\Delta) \equiv 62, \rho_{c}=0.042$, $\mathrm{T}_{\mathrm{c}}=1.153 \mathrm{~K} ;(\mathrm{X}) \equiv 45, \rho_{\mathrm{c}}=0.063, \mathrm{~T}_{\mathrm{c}}=1.196 \mathrm{~K}$;

(d) \#38, $\rho_{c}=0.111, \mathrm{~T}_{c}=1.079 \mathrm{~K}$. 
Data for samples $\# 39$ and $\# 37$ are similar to samples $\$ 62$ and $\# 45$ respectively. 
2. Er Concentration by Auger Analysis

The concentration of $\mathrm{Er}$ in each sample was determined from Auger spectra. A reference alloy of $0.86 \%$ Er in Al was prepared by slowly dissolving the Er into an Al charge in vacuum. The Al and Er were placed together in an aluminum coated Mo boat. The boat was heated very slowly until the Al melted. Once the Er was dissolved, the alloy was kept in the liquid state for about 30 minutes to ensure complete diffusion of the Er. The alloy charge was cut into a thin slab for Auger analysis. Several hundred angstroms were sputtered from the surface of the reference alloy to ensure steady-state surface concentrations. Spectra from three different locations confirmed that the Er was uniformly diffused throughout the sample. The peak-to-peak Auger signal at $131 \mathrm{eV}$ was used to represent the quantity of Er while the signal at 1390 $\mathrm{eV}$ was used for Al. It was determined that the Er concentration was given by

$$
C=(1.1 \pm 0.1)(131 \mathrm{eV} \text { peak } / 1390 \mathrm{eV} \text { peak })
$$

Auger spectra were taken at approximately $100 \AA$ intervals for each of the samples analyzed. The concentration was determined from the weighted average of these spectra.

Table $I$ is a summary of fitted values of $\rho_{c}$ and $T_{c}$ from $\omega_{R}(T)$ data and Er concentration data from Auger analysis. Fig. (8) is a plot of $2 \pi \rho_{c} T_{c}$ vs C. The solid line is a plot of Eq. (18). For Al the parameters needed to evaluate $\Gamma$ are: 58,59 
TABLE I

Pair-Breaking Energy Determined from Relaxation

Frequencies and From Erbium Concentrations

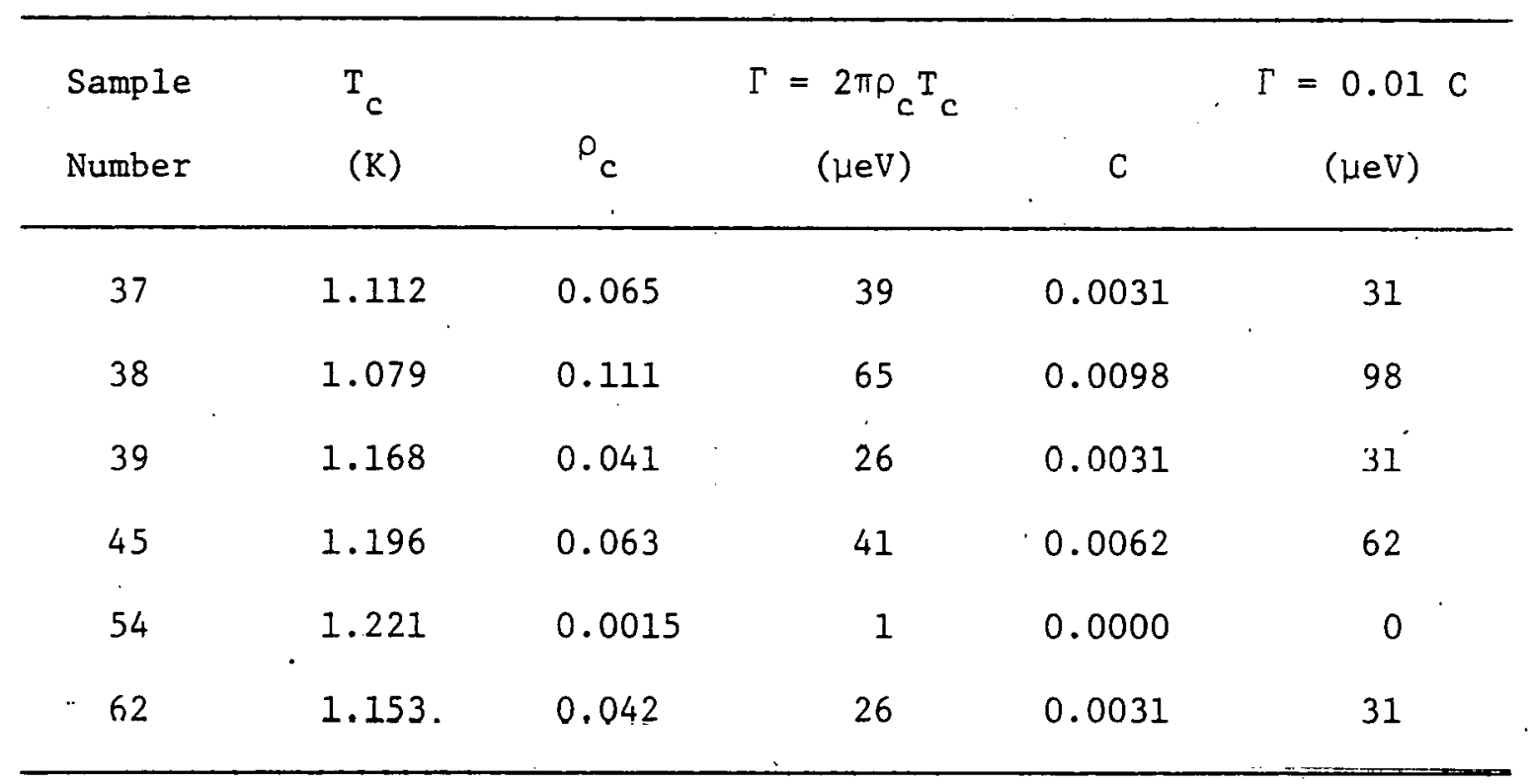




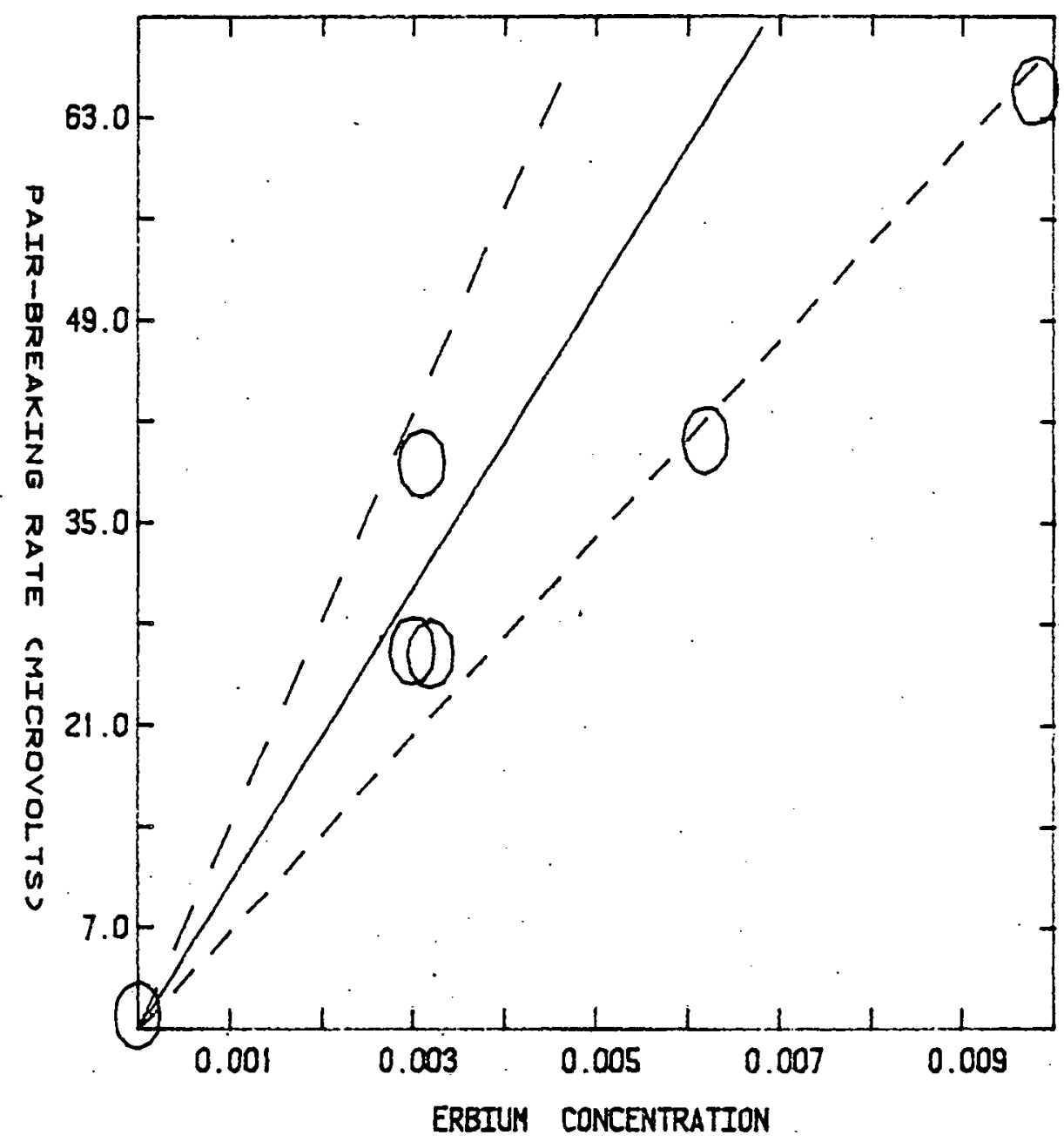

Figure 8. Pair-breaking energy $\Gamma=2 \pi \rho_{c} T_{c}$ plotted vs. Er concentration determined by Auger analysis. Solid line is theory and dashed lines are error limits on the theory as explained in text. 


$$
\left.\begin{array}{l}
J_{S f}=0.11 \pm 0.02 \mathrm{eV} \\
N\left(E_{F}\right)=0.21 \text { states } / \mathrm{eV} \\
g_{J}=6 / 5 \\
J=15 / 2
\end{array}\right\}
$$

With these parameters Eq. (18) becomes

$$
\Gamma=(0.010 \pm 0.004) \mathrm{eV} \times \mathrm{C} .
$$

The error limits on the theory due to the uncertainty in $J_{s f}$ are represented by dashed lines. The statistical error in the data is represented by the size of the plotted symbol. There may be an additional systematic error of $\pm 10 \%$ in the calibration of the Auger spectra.

It should be noted that the Er concentration determined from Auger spectra should be reyalded as the maximum valuc that would contribute to spin-flip scattering. Some of the Er detected may actually be oxidized and thus would be ineffective as a spin-flip scattering site. With this in mind the data points in Fig. (8) arc in remarkable agreement with theory.

Other physical parameters which are determined at temperatures above the Al-Er alloy transition temperature are summarized in Table II. 
TABLE II

Physical Parameters of $\mathrm{Al}_{1-\mathrm{x}^{\mathrm{Er}} \mathrm{x}}$ Electrodes and of Junction Barriers

\begin{tabular}{ccccc}
\hline $\begin{array}{c}\text { Sample } \\
\text { Number }\end{array}$ & $\begin{array}{c}\mathrm{R}_{\mathrm{N}} \\
\text { (ohms) }\end{array}$ & \multicolumn{1}{c}{$\mathrm{R}_{\mathrm{N}} \mathrm{G}_{\mathrm{L}}$} & $\begin{array}{c}\mathrm{Al}_{1-\mathrm{x}} \mathrm{Er}_{\mathrm{x}} \text { electrode } \\
\text { thickness }(\AA)\end{array}$ & $\begin{array}{c}\mathrm{D} \\
\left(\mathrm{cm}^{2} / \mathrm{sec}\right)\end{array}$ \\
\hline 37 & 0.63 & 150 & 1430 & 58 \\
38 & 1.86 & 5,000 & 1100 & 80 \\
39 & 1.27 & 13,000 & 920 & 200 \\
45 & 0.145 & 710 & 780 & 97 \\
54 & 0.103 & 770 & 1010 & 592 \\
62 & 0.053 & 240 & 870 & 471 \\
\hline
\end{tabular}

The area of each junction is $(0.27 \mathrm{~mm})^{2}$. The diffusion constant $D$ is determined from the data and Eqs. (4) and (16). $R_{N}$ is the normal tunneling resistance and $G_{L}$ is the leakage conductance. 
B. DATA FOR $\mathrm{T}<\mathrm{T}_{\mathrm{C}}$

In this section we will present the data obtained from five junctions at temperatures below the transition temperature. Many more samples were examined, but were found unsuitable for analysis because of the quality (leaky or shorted), the normal resistance (too high or too low), or the transition temperature (too low) of the junction. In addition, several of the alloys had such low Er concentrations that pair-breaking effects were unobservable. of the five samples examined, one contained no Er, two exhibited a pair-breaking energy of $26 \mu \mathrm{V}$, and two had a pair-breaking energy of about $40 \mu \mathrm{V}$. This covers a wide range of pair-breaking energy while allowing a check for consistency.

Actual excess current-voltage characteristics as well as the eigenfrequency fitting parameters are presented. Comparison to the theories of Orbach and Entin-Wohlman, Dinter, and Schön and Ambegaokar are made. Data to test the Schön-Ambegaokar theory for the transverse mode in the current carrying state is also given. 


\section{Excess Current}

Fig. (9) contains a set of $I_{e x}-V$ curves for sample $\$ 54$ $(\Gamma=0)$. The magnetic field is the same for all curves: $\left(\mathrm{Dk}^{2}=27 \mu \mathrm{V}\right)$ but each curve is taken at a different temperature. One can clearly see the single feature at $T-T_{c}(\varepsilon=0)$ develop into three features with increasing $\varepsilon$. Notice that the dc Josephson current could not be entirely suppressed for all temperatures at fixed magnetic fields. Because of this some curves contain irregular features or missing data near $V=0$.

The effect on the excess current due to changing the magnetic field can be seen by examining Figs. (10)-(20). These figures are constructed in the same manner as Fig. (9) but are obtained from. samples $\# 39$ and $\# 62$. The pair-breaking energy for both samples is about $26 \mu \mathrm{V}$. The normal tunneling resistance for sample 非 62 is about 30 times less than that of sample $\$ 39$ resulting in a three order-ofmagnitude increase in the excess current. Figs. (13) and (15) represent $\mathrm{Dk}^{2}=20 \mu \mathrm{V}$ for samples $\# 39$ and $\# 62$ respectively. As expected they are quite similar except for the three order-ofmagnitude scale factor in the current axis. Several qualitative results are observable from this sequence of figures. The low frequency "shoulder" is shifted to higher frequencies as $\mathrm{Dk}^{2}$ is increased. With respect to the transverse mode, there is no propagation (observable as a sharpening of the mid-frequency peak) for small values of $\mathrm{Dk}^{2}$ such as in Figs. (11) and (12). For larger values of $\mathrm{Dk}^{2}$ such as in Figs. (13) to (i7) notice the onset of propagation at $\varepsilon \simeq 0.008$. This corresponds to $\Delta \simeq \Gamma$. For very 


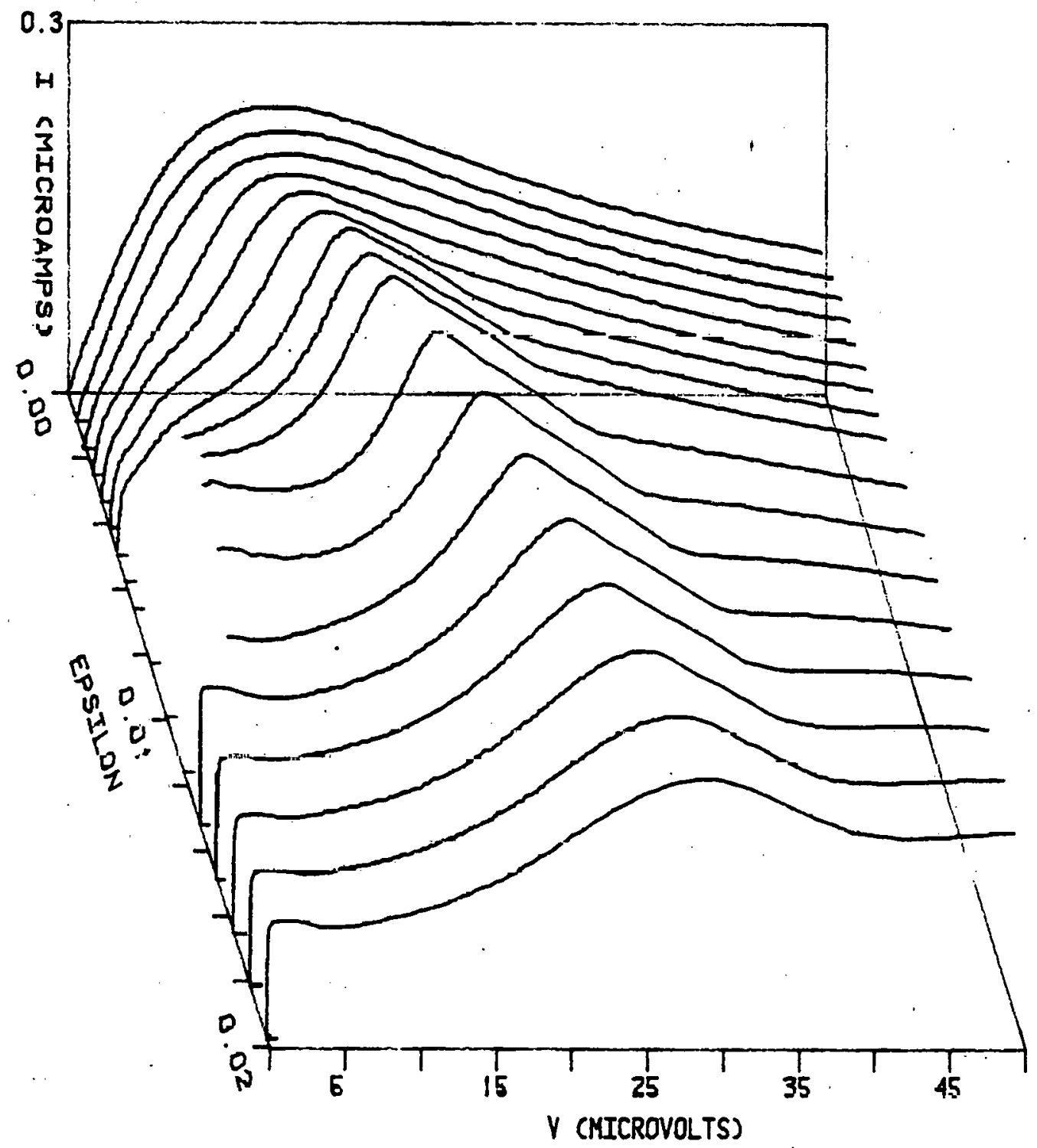

Figure 9. Excess current for $\# 54: \quad \Gamma=0, \mathrm{H}=29.8$ Oe, $\mathrm{Dk}^{2}=27 \mu \mathrm{V}$, $T_{c}=1.214 \mathrm{~K}$, and $R_{N}=0.103$ ohms. 


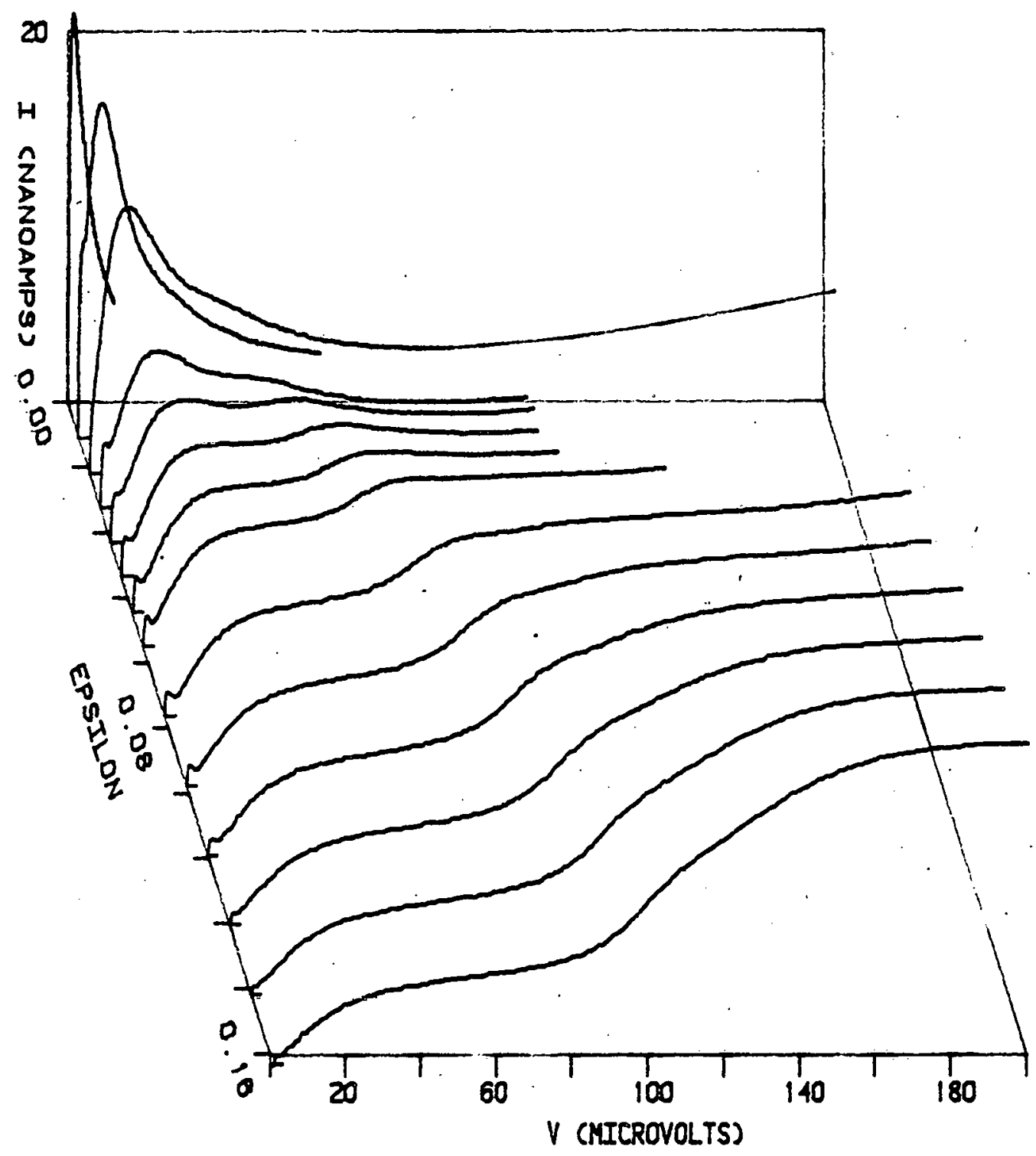

Figure 10. Excess current for 非 39 : $\Gamma=26 \mu \mathrm{V}, \mathrm{H}=15.8 \mathrm{Oe}$, $\mathrm{Dk}^{2}=3.7 \mu \mathrm{V}, \mathrm{T}_{\mathrm{c}}=1.170 \mathrm{~K}$ and $\mathrm{R}_{\mathrm{N}}=1.27$ uhus. 


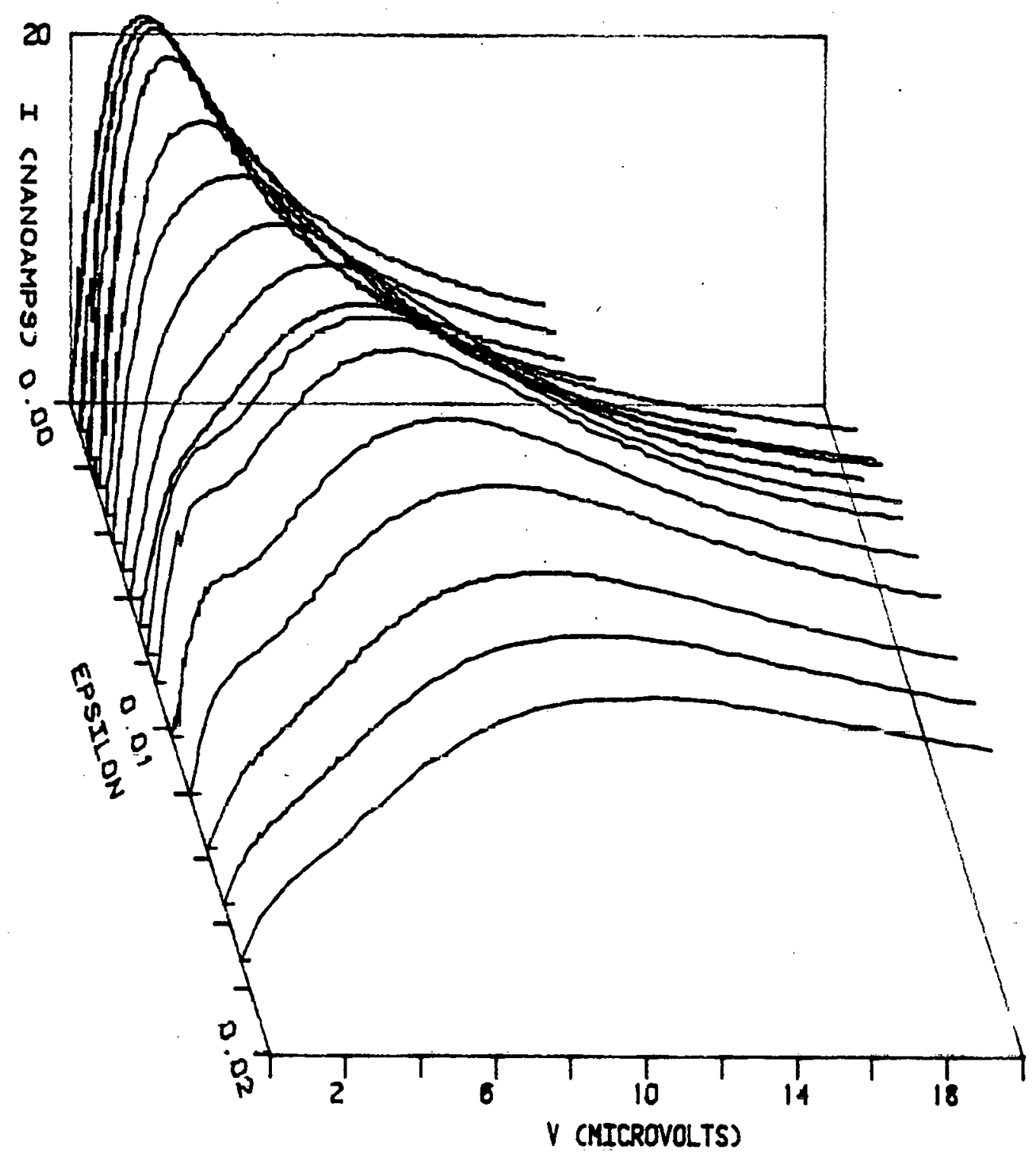

Figure 11. Detail of excess current for $\# 39: \quad \Gamma=26 \mu \mathrm{V}, \mathrm{H}=15.8 \mathrm{Oe}$, $\mathrm{Dk}^{2}=3.7 \mu \mathrm{V}, \mathrm{T}_{\mathrm{C}}=1.170 \mathrm{~K}$ and $\mathrm{R}_{\mathrm{N}}=1.27 \mathrm{ohms}$. 


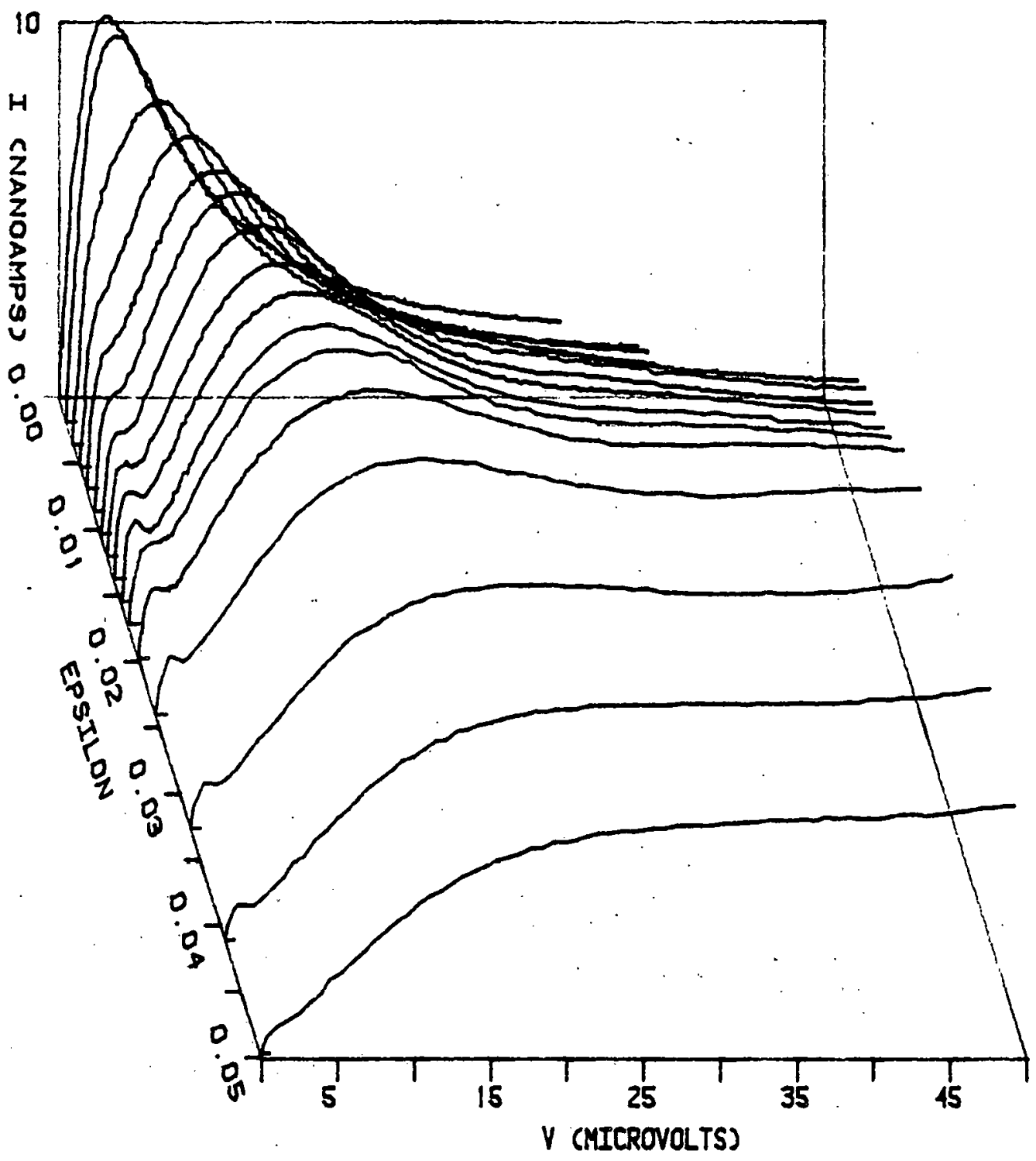

Figure 12. Excess current for $\# 39: \Gamma=26 \mu \mathrm{V}, \mathrm{H}=24.5 \mathrm{Oe}$, $\mathrm{Dk}^{2}=6.0 \mu \mathrm{V}, \mathrm{T}_{\mathrm{c}}=1.168 \mathrm{~K}$ and $\mathrm{R}_{\mathrm{N}}=1.27$ ohms. 


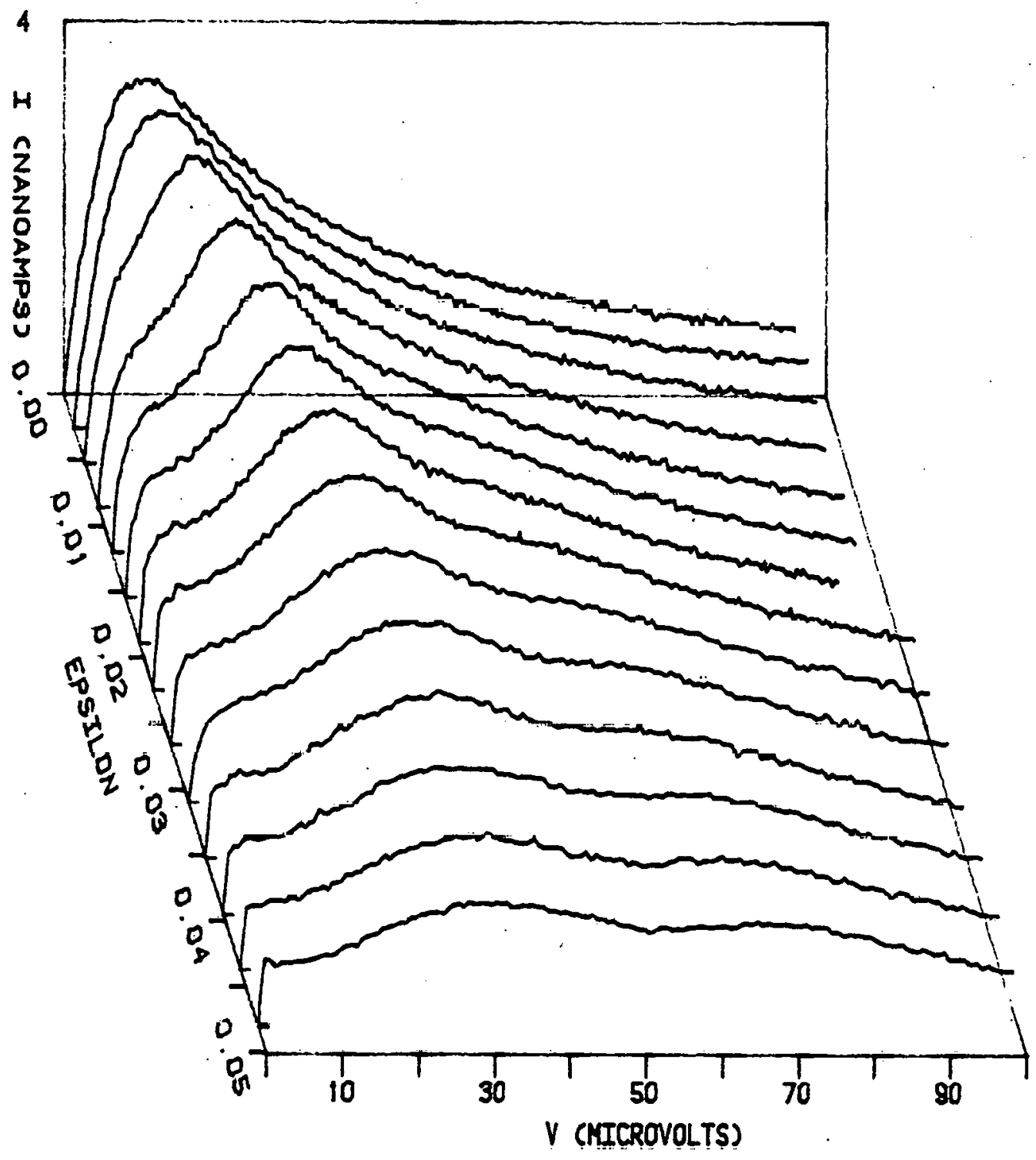

Figure 13. Excess current for $\$ 39: \quad \Gamma=26 \mu \mathrm{V}, \mathrm{H}=46.6 \mathrm{Oe}$, $\mathrm{Dk}^{2}=20.6 \mu \mathrm{V}, \mathrm{T}_{\mathrm{c}}=1.166 \mathrm{~K}$, and $\mathrm{R}_{\mathrm{N}}=1.27 \mathrm{ohms}$. 


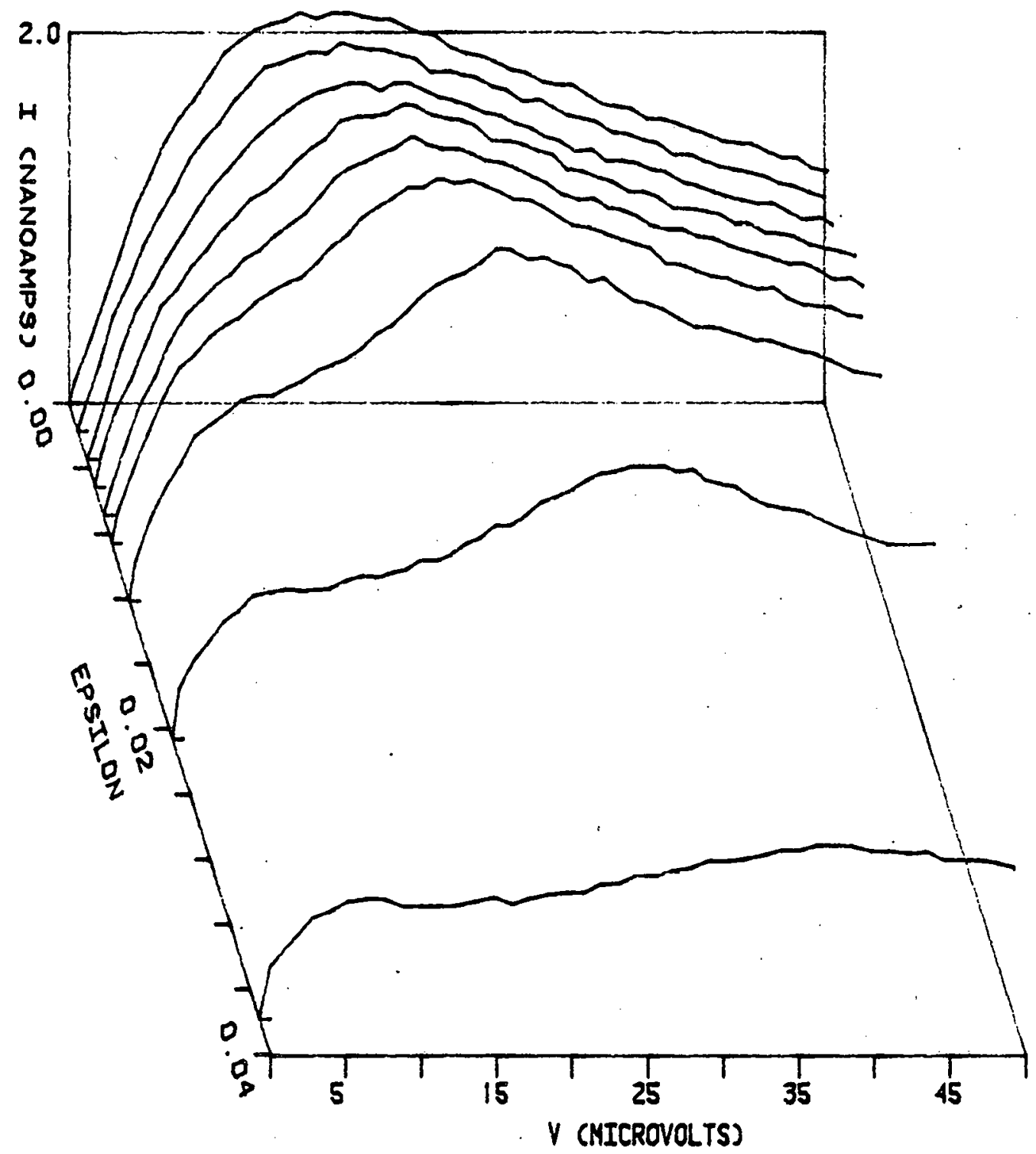

Figure 14. Excess current for $\# 39: \quad \Gamma=26 \mu \mathrm{V}, H=60.0 \mathrm{Oe}$, $\mathrm{Dk}^{2}=33.5 \mu \mathrm{V}, \mathrm{T}_{\mathrm{c}}=1.164 \mathrm{~K}$, and $\mathrm{R}_{\mathrm{N}}=1.27$ ohms. 


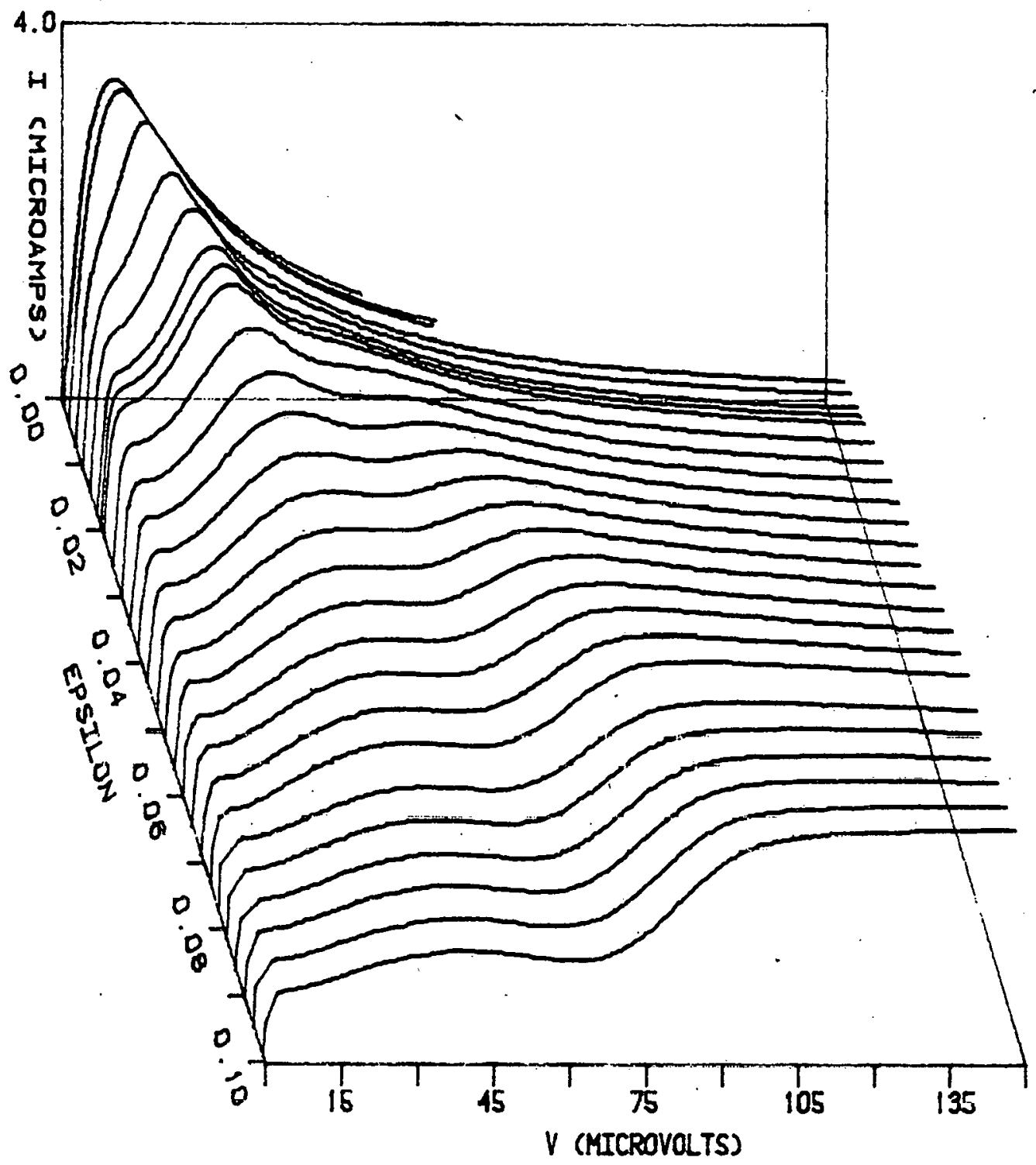

Figure 15. Excess current for $\# 62$ : $\Gamma=26 \mu \mathrm{V}, \mathrm{H}=31.2 \mathrm{Oe}$, $\mathrm{Dk}^{2}=20.21 \mathrm{VV}, \mathrm{T}_{\mathrm{c}}=1.1 .55 \mathrm{~K}$, and $\mathrm{R}_{\mathrm{N}}=0.053 \mathrm{ohms}$. 


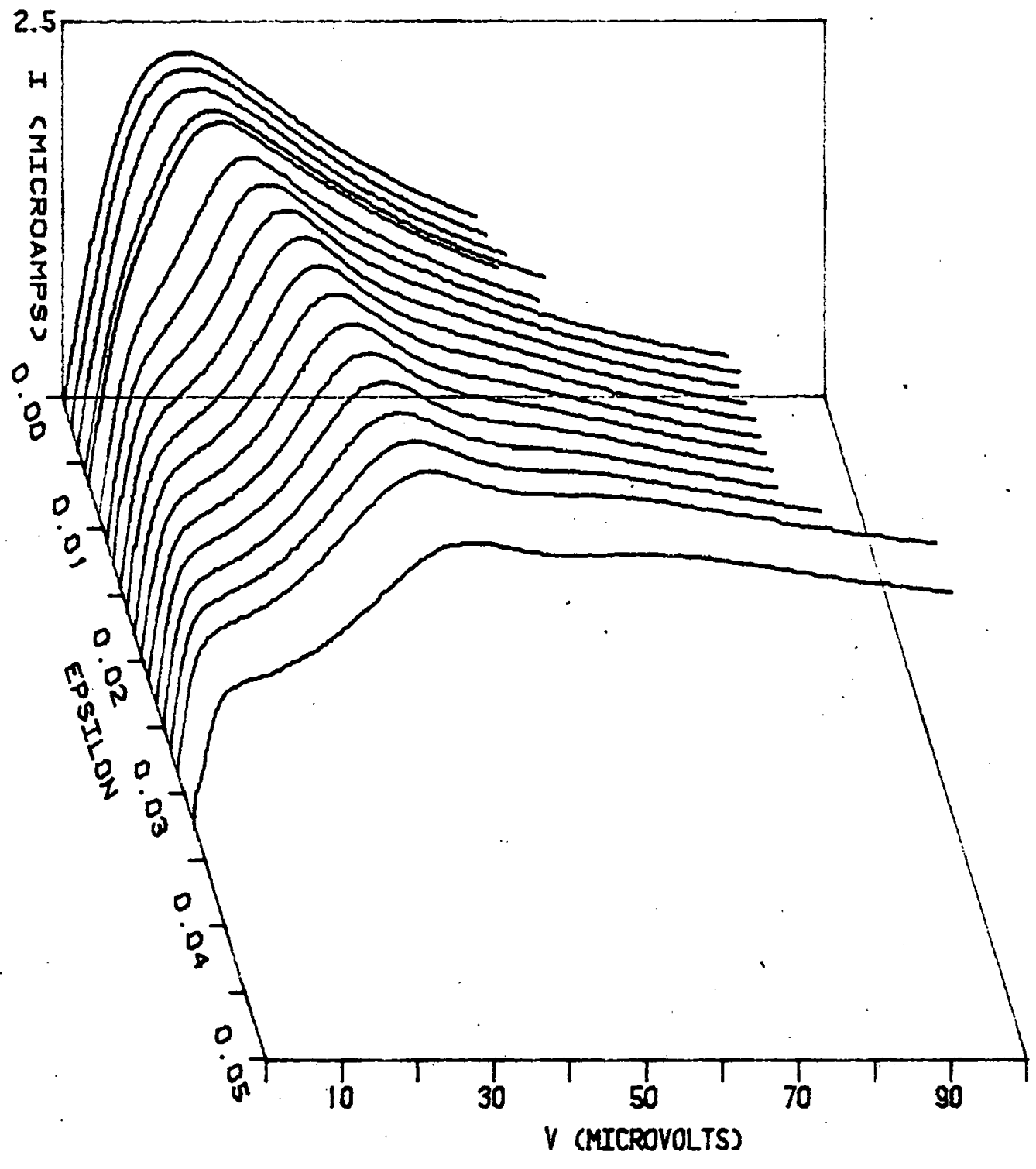

Figure 16. Excess current for 非62: $\Gamma=26 \mu \mathrm{V}, \mathrm{H}=38.9$ Oe, $\mathrm{Dk}^{2}=30.5 \mu \mathrm{V}, \mathrm{T}_{\mathrm{c}}=1.153 \mathrm{~K}$, and $\mathrm{R}_{\mathrm{N}}=0.053 .0 \mathrm{hms}$. 


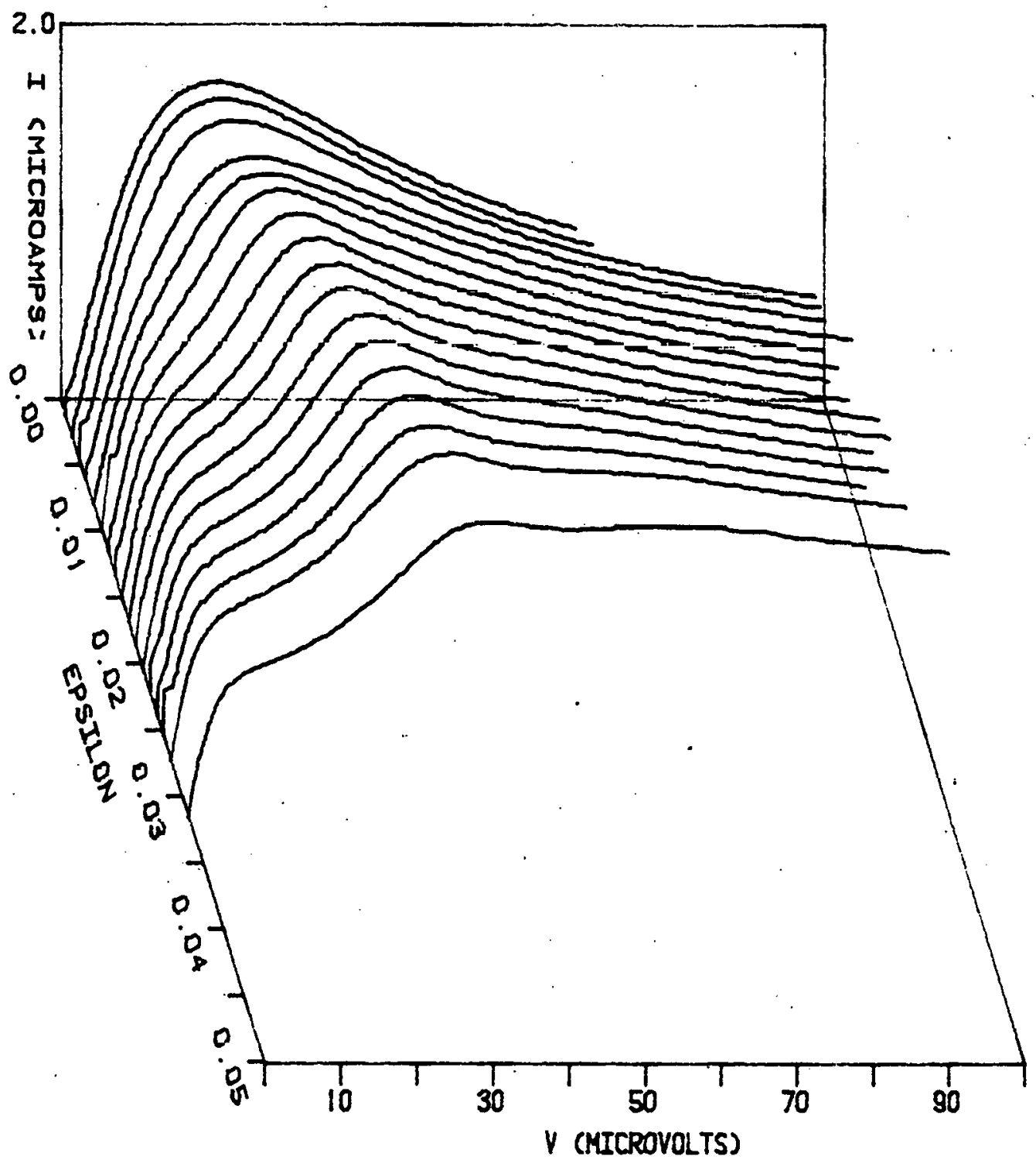

Figure 17. Excess current for $\$$ 62: $\Gamma^{\prime}=26 \mu \mathrm{V}, \mathrm{H}=44.0 \mathrm{Oe}$, $\mathrm{Dk}^{2}=39.0 \mu \mathrm{V}, \mathrm{T}_{\mathrm{C}}=1.152 \mathrm{~K}$, and $\mathrm{R}_{\mathrm{N}}=0.053$ ohms. 


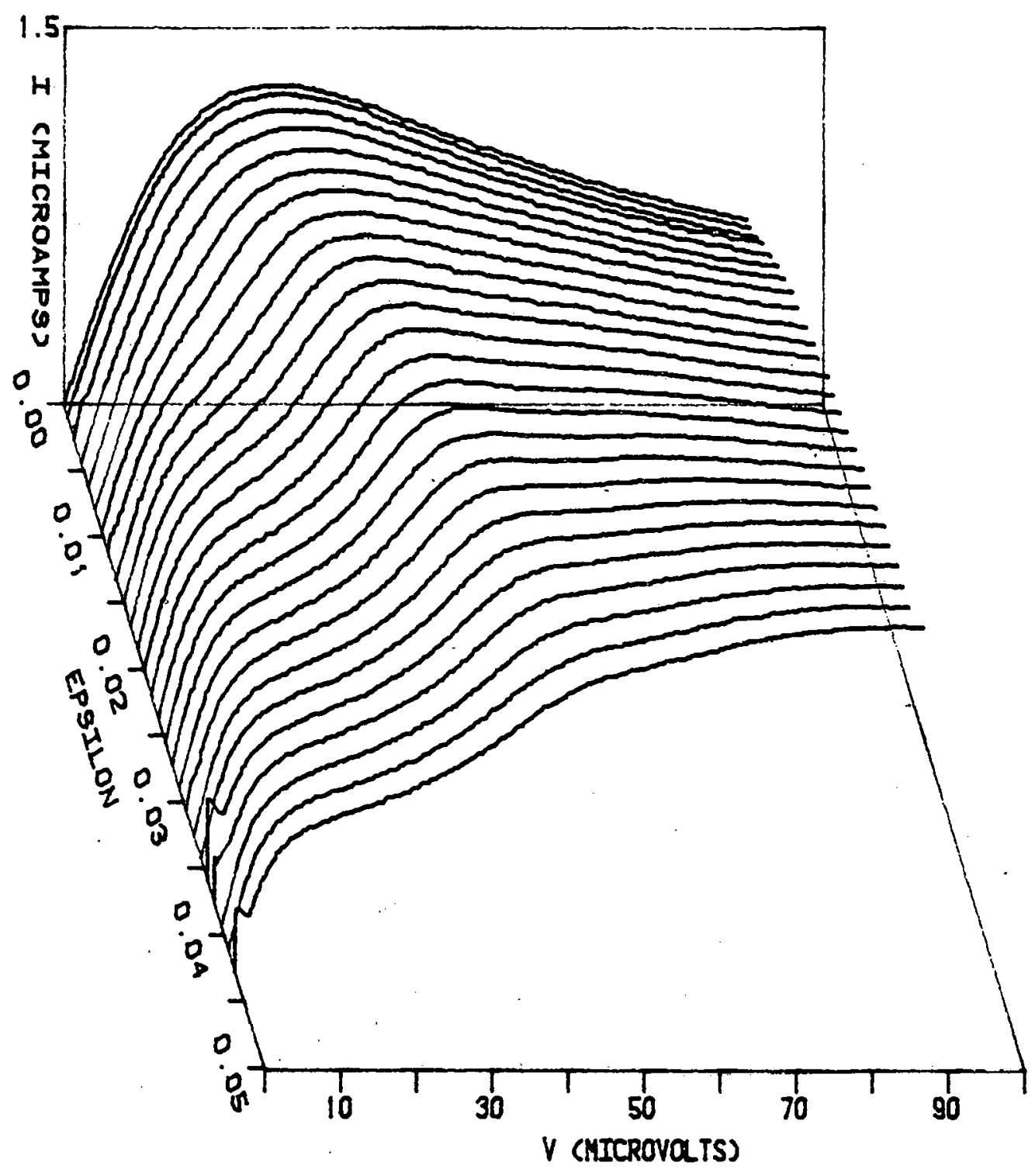

Figure 18. Excess current for $\$ 62: \quad \Gamma=26 \mu \mathrm{V}, \mathrm{H}=52.3 \mathrm{Oe}$, $\mathrm{Dk}^{2}=54.7 \mu \mathrm{V}, \mathrm{T}_{\mathrm{C}}=1 \cdot 1.50 \mathrm{~K}$, and $\mathrm{R}_{\mathrm{N}}=0.053 \mathrm{ohms}$. 


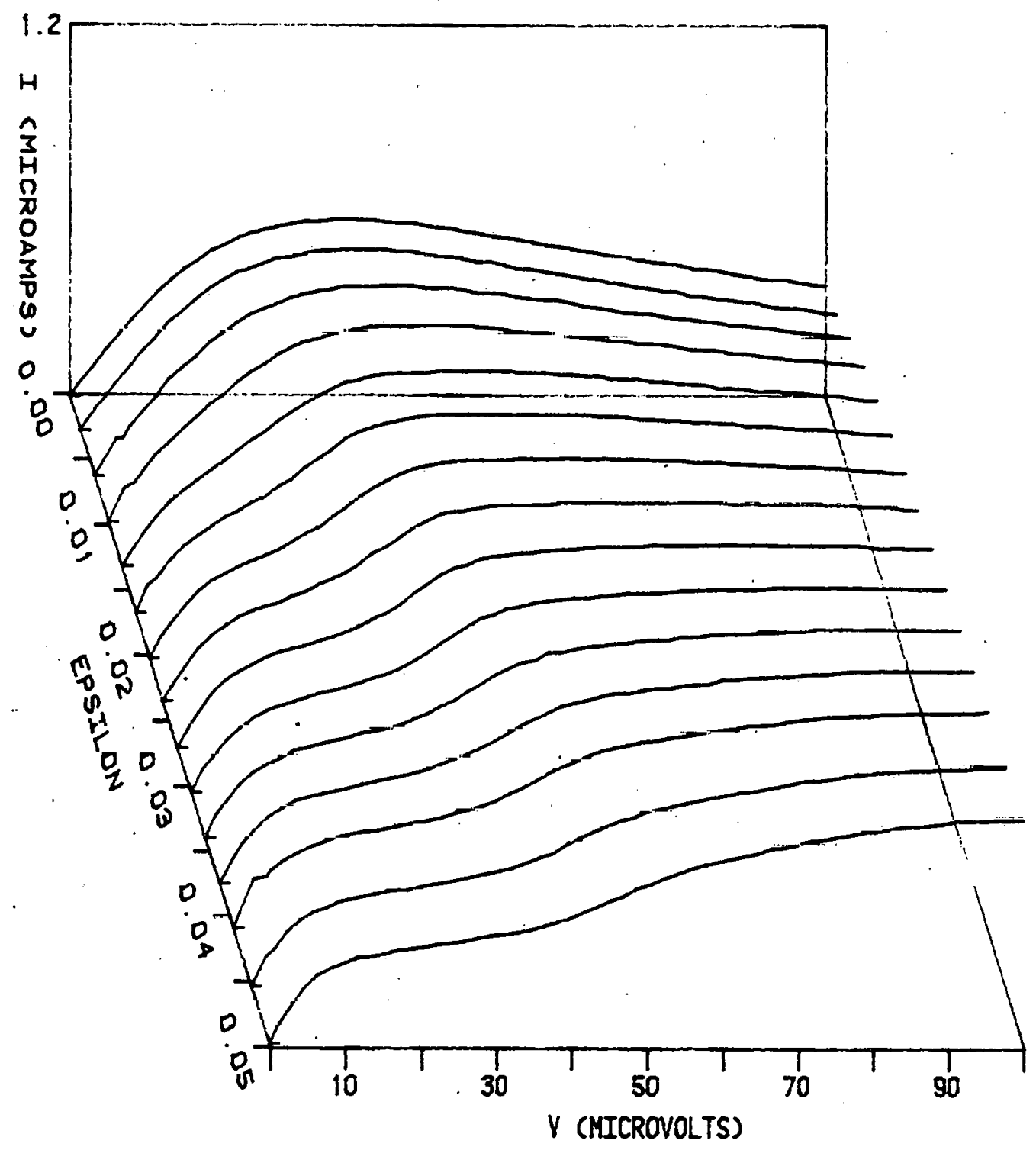

Figure 19. Excess current for $\# 62: \quad \Gamma=26 \mu \mathrm{V} ; \mathrm{H}=60.0 \mathrm{Oe}$, $\mathrm{Dk}^{2}=71.5 \mu \mathrm{V}, \mathrm{T}_{\mathrm{C}}=1.147 \mathrm{~K}$, and $\mathrm{R}_{\mathrm{N}}=0.053$ ohms. 


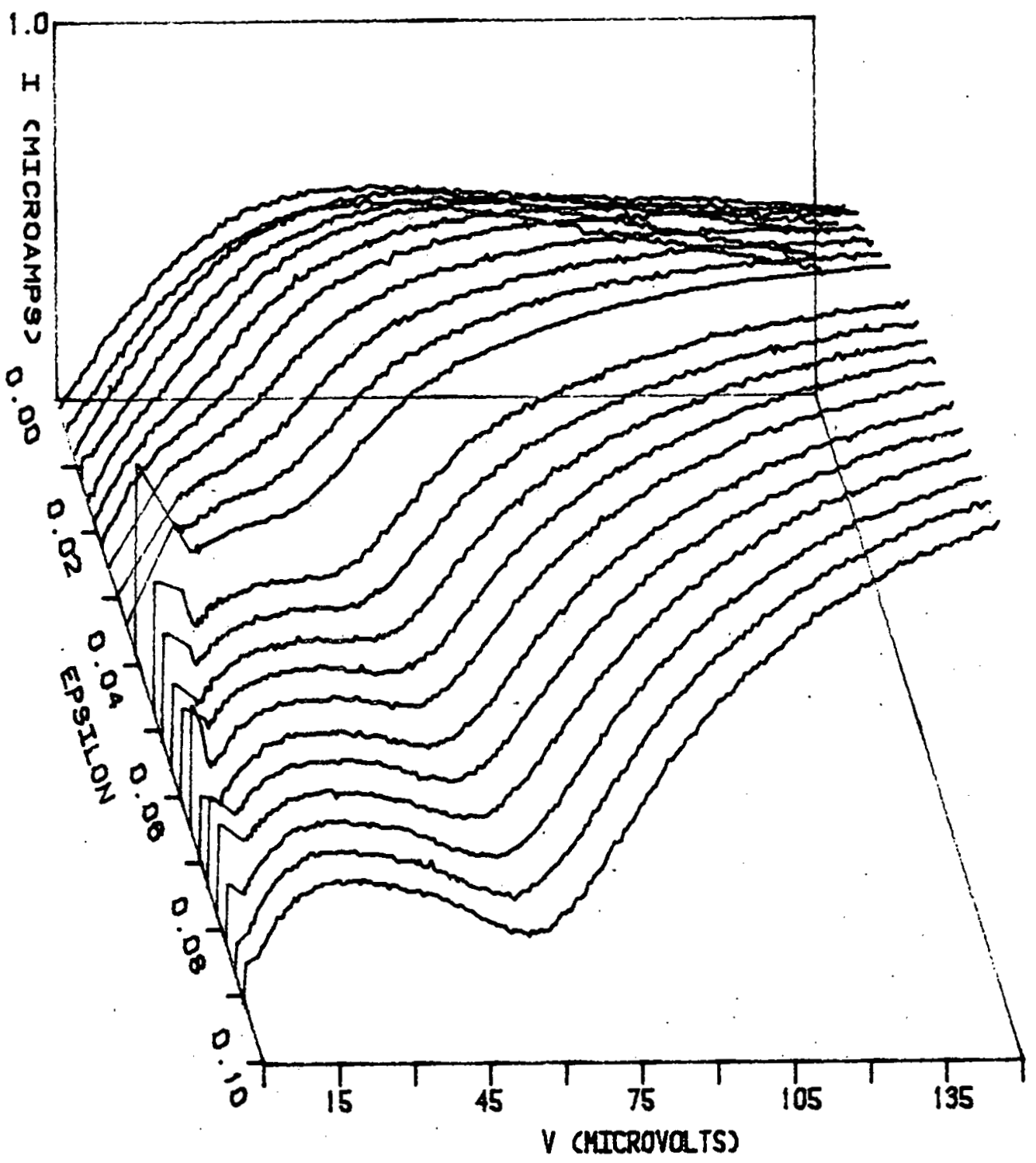

Figure 20. Excess current for $\$ 62$ : $\Gamma=26 \mu \mathrm{V}, \mathrm{H}=80.0 \mathrm{Oe}$, $\mathrm{Dk}^{2}=120 . \mu \mathrm{V}, \mathrm{T}_{\mathrm{C}}=1.140$, and $\mathrm{R}_{\mathrm{N}}=0.053 \mathrm{ohms}$. 
large values of $\mathrm{Dk}^{2}$ as in Figs. (18) to (20) the transverse mode is lost in the gap peak. This may be the result predicted by Holstein, Orbach and Kulik ${ }^{48}$ for $\mathrm{Dk}^{2} \gg \Delta$ (see Eqs. (167) to (169)).

Samples $\# 37$ (Fig. (21)) and \#45 (Fig. (22)) both have a pairbreaking energy of about 40 $\mathrm{V}$. One can see that propagation of the transverse mode is again delayed to $\Delta \simeq \Gamma(\varepsilon \simeq 0.015)$. The other qualitative result associated with increased pair-breaking Is Llial Llie gap peak is bruadened. 


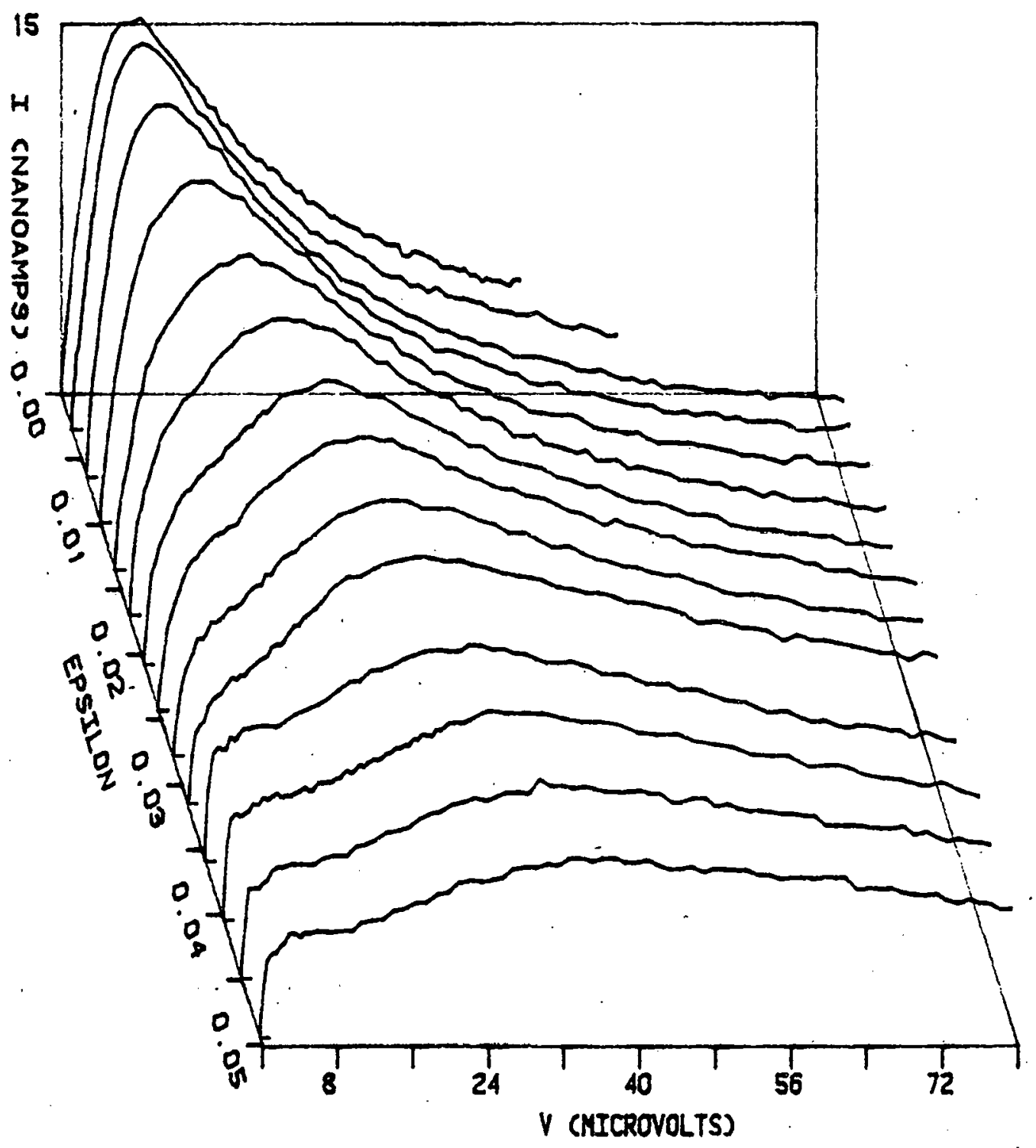

Figure 21. Excess current for $\$ 37$ : $\Gamma=39 \mu \mathrm{V}, \mathrm{H}=60.0 \mathrm{De}$, $\mathrm{Dk}^{2}-14.7 \mu \mathrm{V}, \mathrm{T}_{\mathrm{c}}=1.115 \mathrm{~K}$, and $\mathrm{R}_{\mathrm{N}}=0.63$ ohms. 


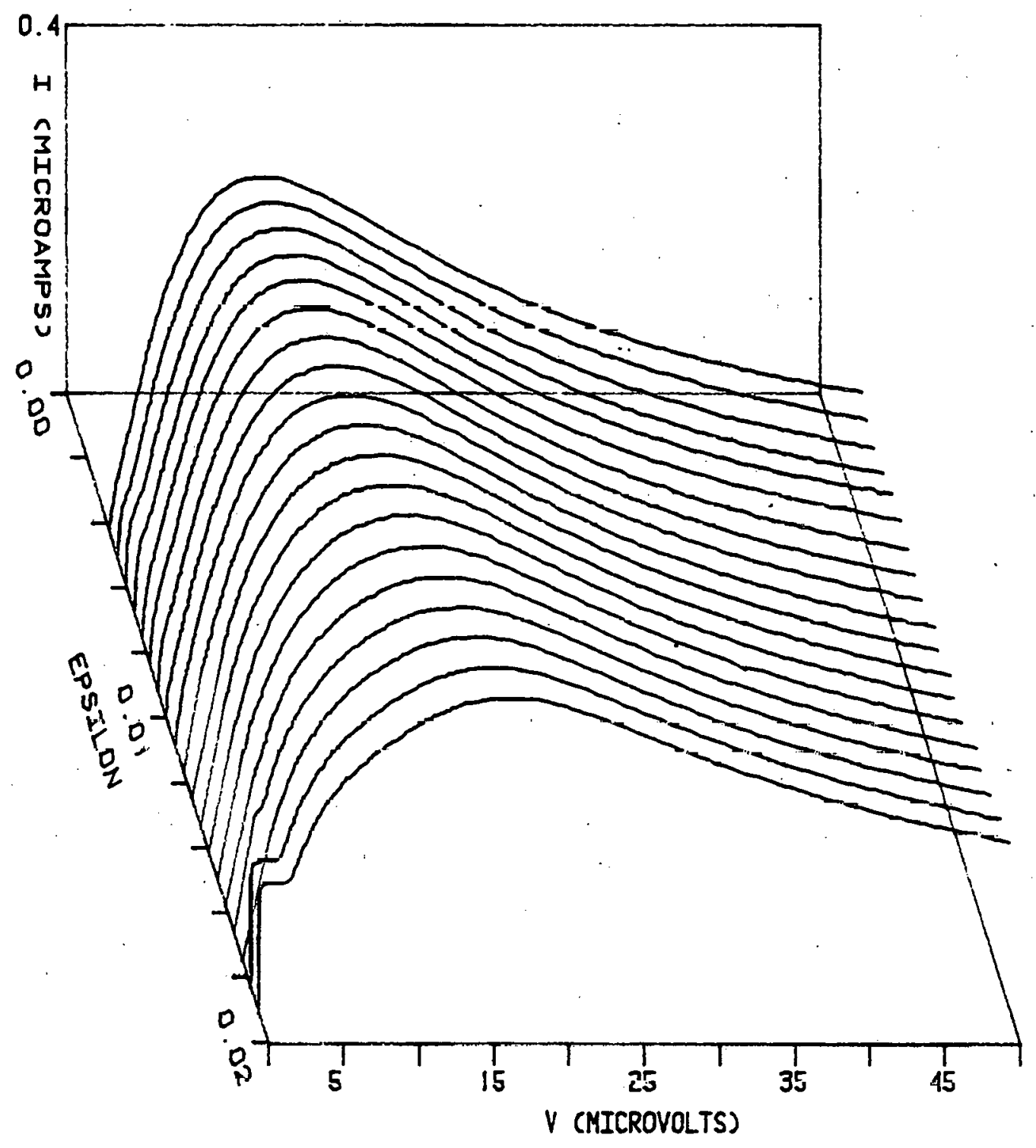

Figure 22. Excess current for $\$ 45: \Gamma=41 \mu \mathrm{V}, \mathrm{H}=75.6 \mathrm{Oe}$, $\mathrm{Dk}^{2}=21 \mu \mathrm{V}, \mathrm{T}_{C}=1.203 \mathrm{~K}$, and $\mathrm{R}_{\mathrm{N}}=0.145 \mathrm{ohms}$. 
2. Eigenfrequencies

The qualitative features noted above can be made quantitative by extracting the eigenfrequencies in the manner discussed in Section B of Chapter IV. This will also facilitate comparison to the eigenfrequencies derived by the theorist.

Let us begin by discussing the gap peak which we believe can be identified with the high frequency longitudinal mode. In Figs. (23) to (28) we plot the real and imaginary parts of the eigenfrequency determined from the fitting parameters (see Eqs. (177) to (179)). It should be kept in mind that one drawback of the function chosen to represent the gap peak is that it can only describe a mode in which $\operatorname{Re}(\omega)>\operatorname{Im}(\omega)$. Superimposed on the experimental eigenfrequencies are the real and imaginary parts of Eq. (124), which is the eigenfrequency of the longitudinal mode predicted by the Schön-Ambegaokar theory. The agreement ranges from good to excellent, especially for moderate values of $\mathrm{Dk}^{2}$ and for $\Delta>\Gamma$. The theory of Orbach and Entin-Wohlman from Eqs. (57) and (64) is superimposed in Figs. (23) and (29). They predict an imaginary frequency which nearly coincides with the real part of the measured frequency. Dinter derives a similar result in Eq. (105).

We have derived low frequency dispersion relations for the longitudinal mode from the Orbach Entin-Wohlman (Eqs. (59) and (65)) and Dinter (Eq. (101)) theories. These are superimposed on the data for sample $\#_{62}$ in Figs. (30) and (31) for four values of $\mathrm{Dk}^{2}$. Both theories are consistent with the data for small values of $\nu k^{2}$. It appears that Dinter's $\Gamma$-dependent expression is also in 


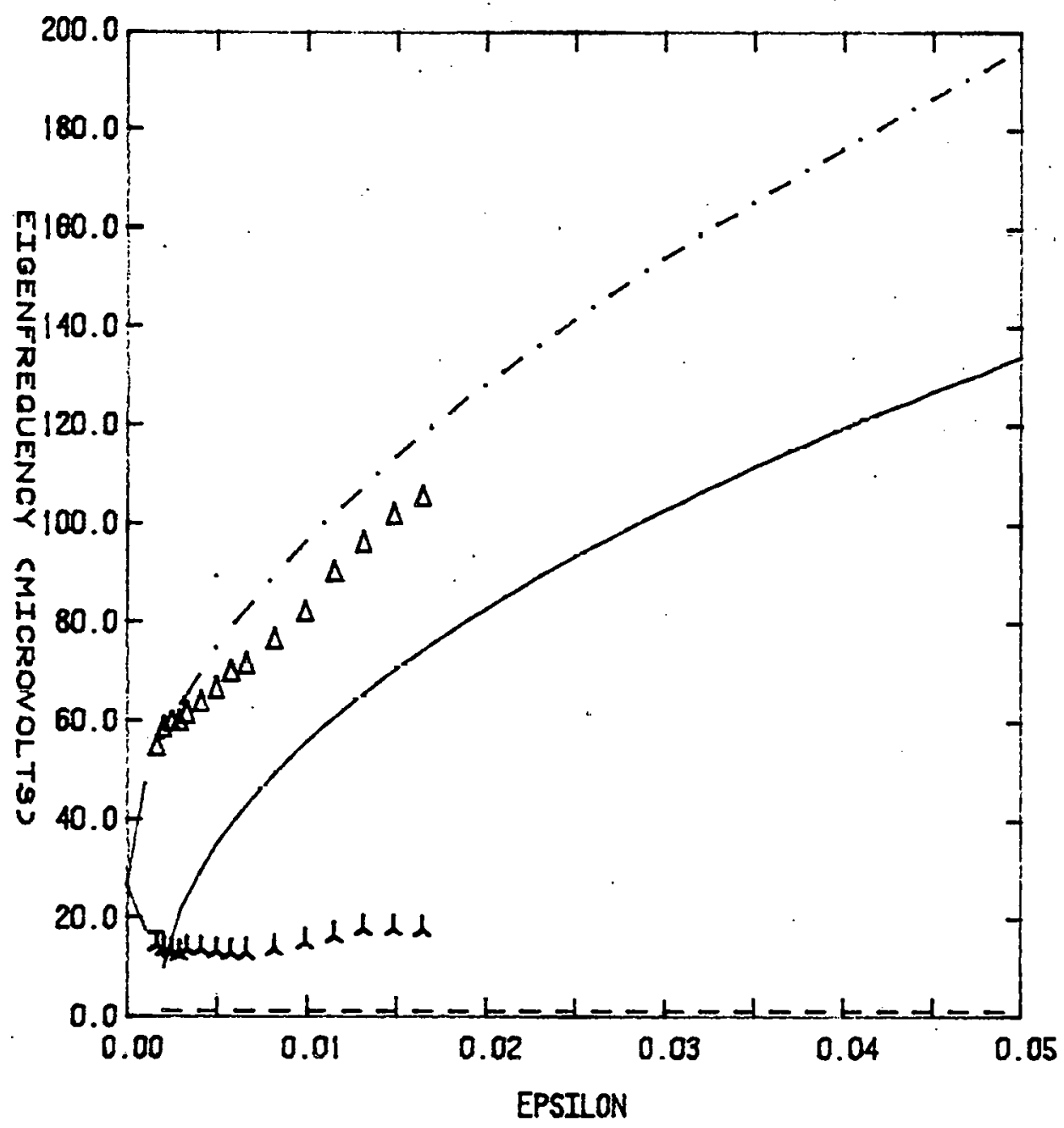

Figure 23. Data for real $(\Delta)$ and imaginary ( $\lambda$ ) parts of the gap mode for $\$ 54$ with $\Gamma=0, \mathrm{Dk}^{2}=27 \mu \mathrm{V}$, and $\mathrm{T}_{\mathrm{c}}=1.214 \mathrm{~K}$. OEW theory (dot-dashed) for pure imaginary highfrequency L-mode. SA theory for real (sol1d) and imaginary (dashed) part of L-mode. 


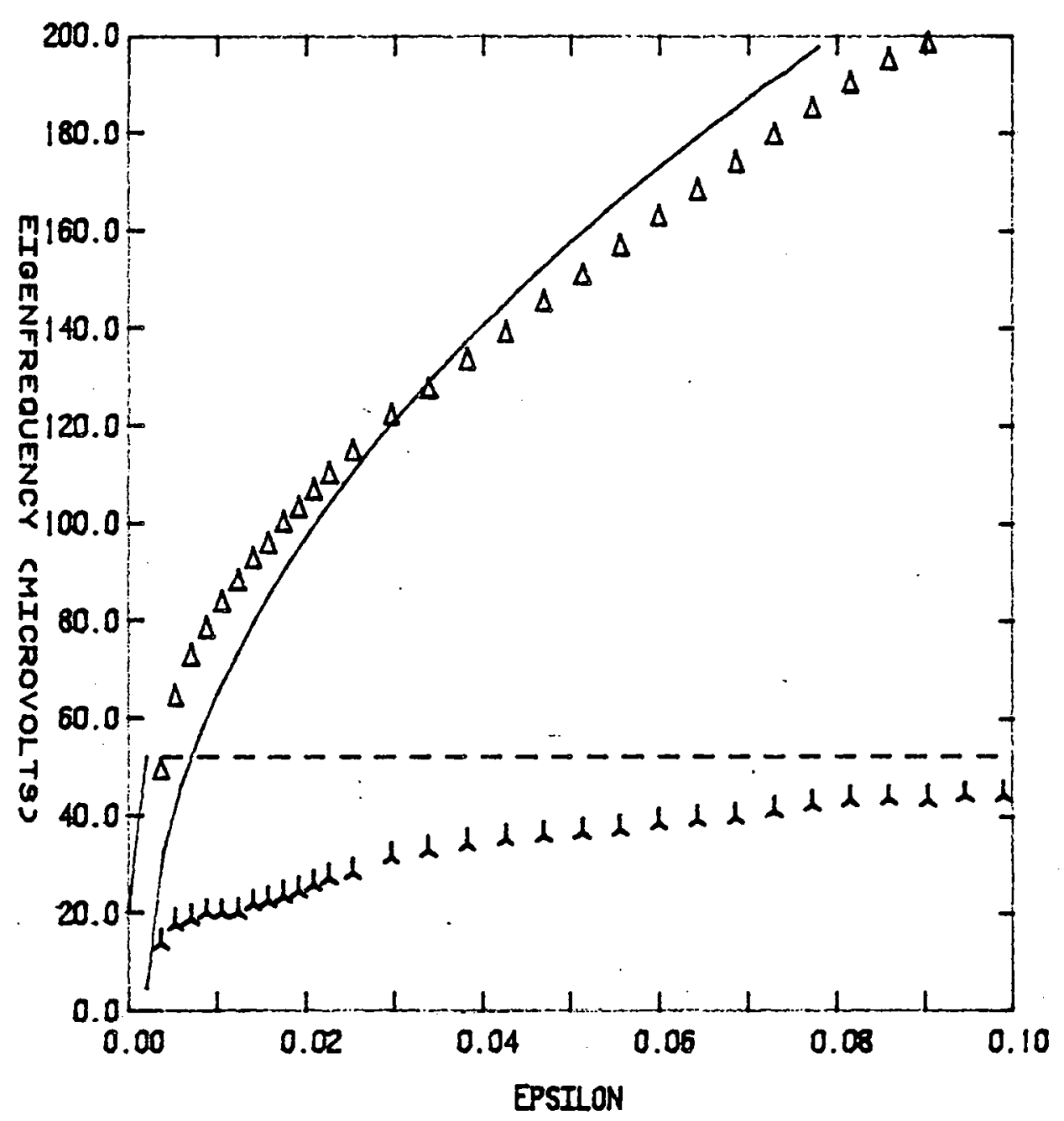

Figure 24. Data for real ( $\Delta$ ) and imaginary ( $(\lambda)$ parts of the gap mode for $\$$ 非 62 with $\Gamma=26 \mu \mathrm{V}, \mathrm{Dk}^{2}=20.2 \mathrm{~V}$, and $\mathrm{T}_{\mathrm{c}}=1.155 \mathrm{~K}$. SA theory for real (solid) and imaginary (dashed) part of L-mode. 


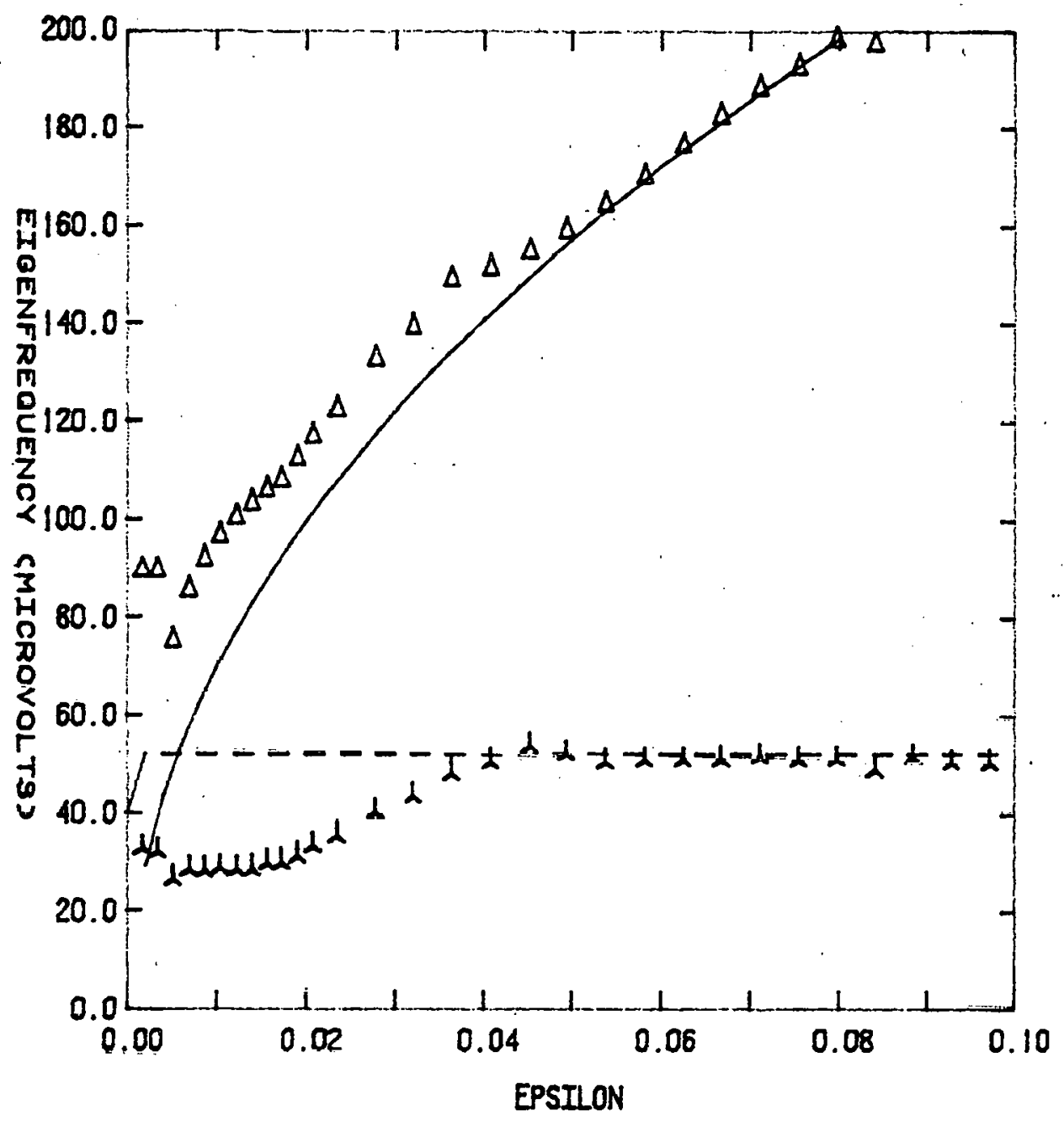

Figure 2b. Data for real $(\Delta)$ and imaginary $(\lambda)$ parls of the gap mode for $\# 62$ with $\Gamma=26 \mu \mathrm{V}, \mathrm{Dk}^{2}=39.0 \mu \mathrm{V}$, and $\mathrm{T}_{c}=1.152 \mathrm{~K}$. SA theory for real (solid) and Imaginary (dashed) part of L-mode: 


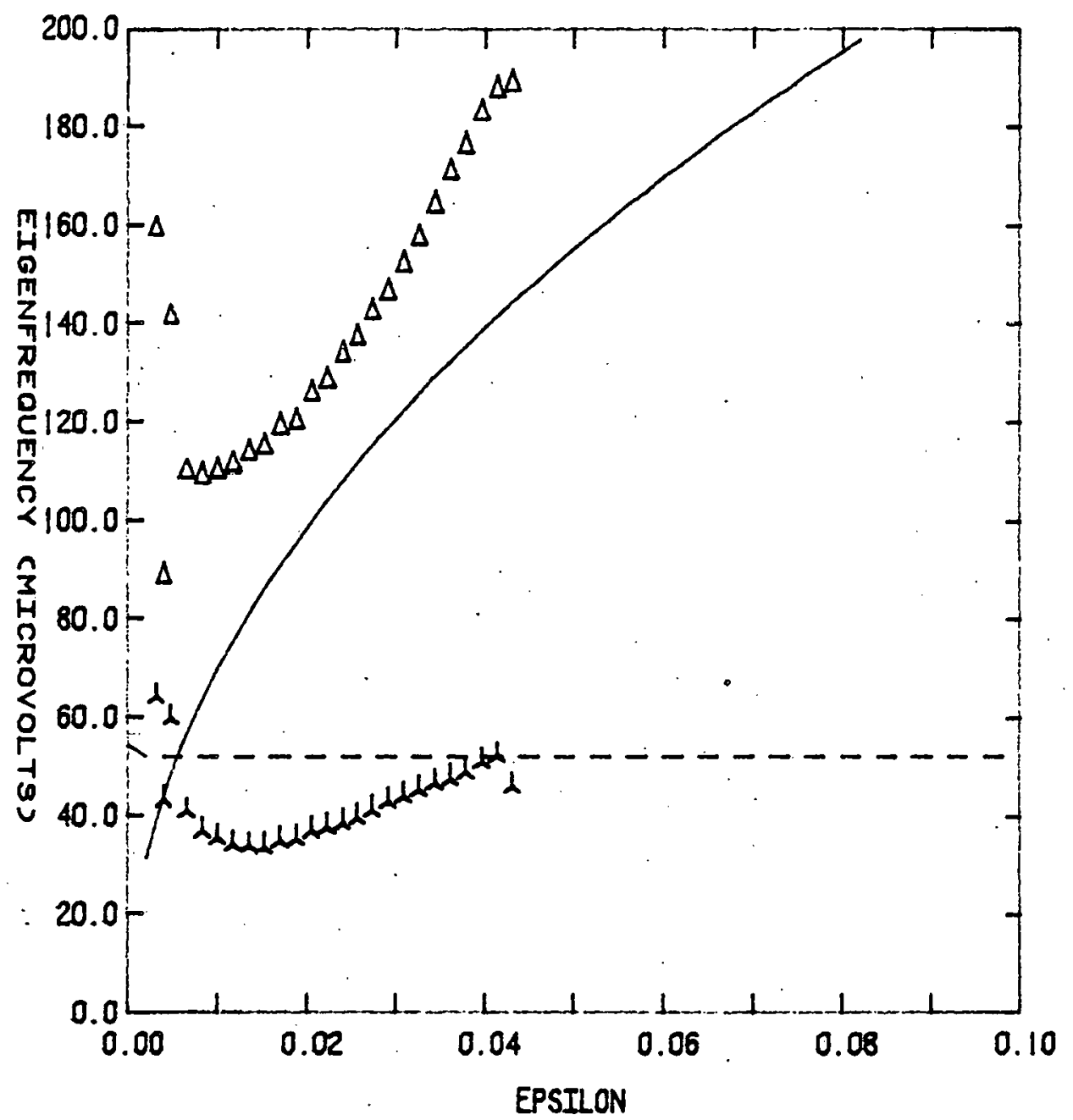

Figure 26. Data for real $(\Delta)$ and imaginary ( $(\lambda)$ parts of the gap mode for $\$$ 非 62 with $\mathrm{I}^{\prime}=26 \mu \mathrm{V}, \mathrm{Dk}^{2}=54.7 \mu \mathrm{V}$, and $\mathrm{T}_{\mathrm{c}}=1.150 \mathrm{~K}$. SA theory for real (solid) and imaginary (dashed) part of L-mode. 


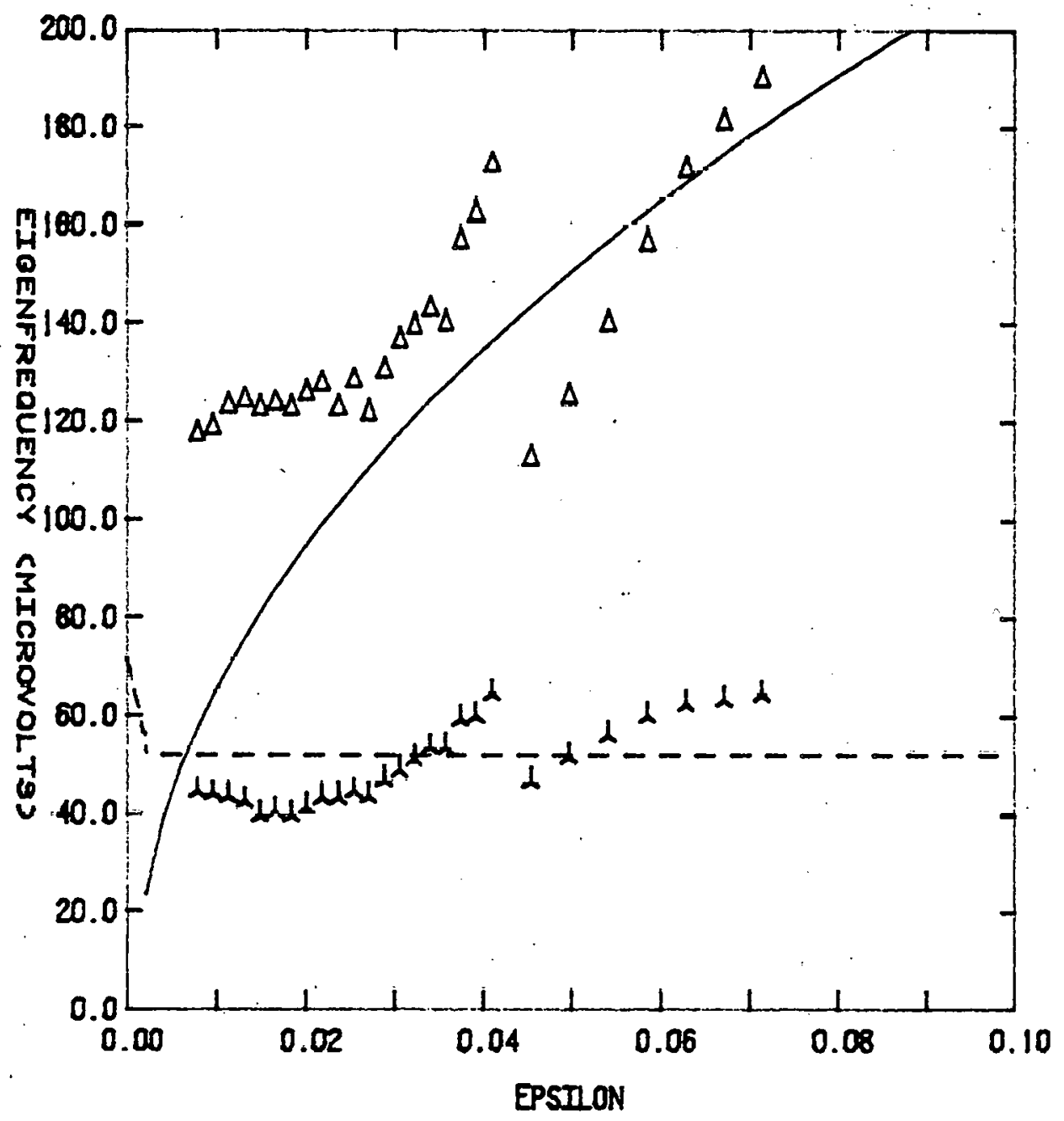

Figure 27. Data for real $(\Delta)$ and imaginary ( $(\lambda)$ parts of the gap mode for 1 k6 with $\Gamma=26 \mu \mathrm{V}, \mathrm{Dk}^{2}=71.5 \mu \mathrm{V}$, and $\mathrm{T}_{\mathrm{C}}=1.147 \mathrm{~K}$. SA theory for real (solid) and imaginary (dashed) part of L-mode. 


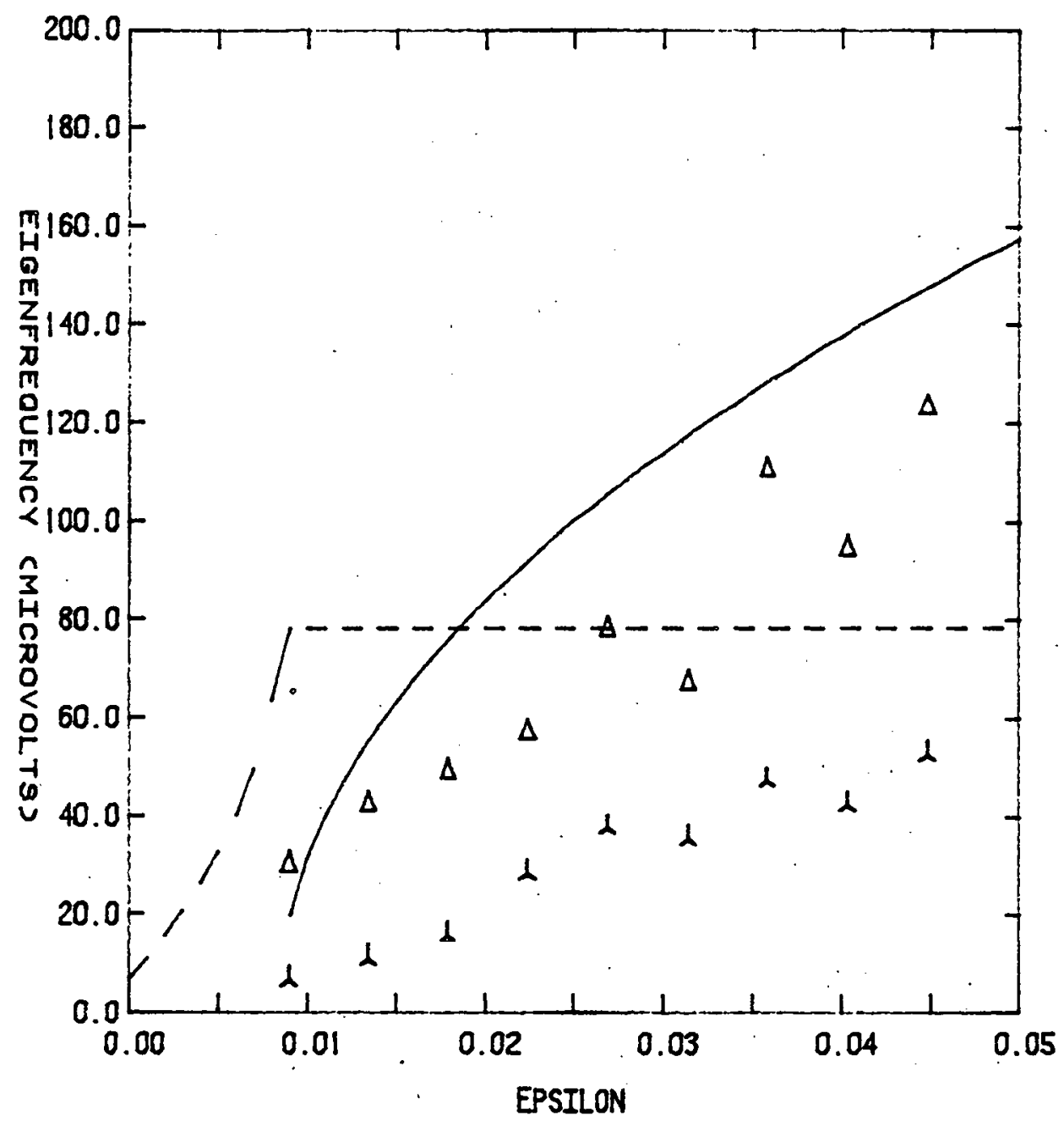

Figure 28. Data for real $(\Delta)$ and imaginary ( $(\lambda)$ parts of the gap mode for $\# 37$ with $\Gamma=39 \mu \mathrm{V}, \mathrm{Dk}^{2}=14.7 \mu \mathrm{V}$, and $\mathrm{T}_{\mathrm{c}}=1.115 \mathrm{~K}$. SA theory for real (solid) and imaginary (dashed) part of L-mode. 


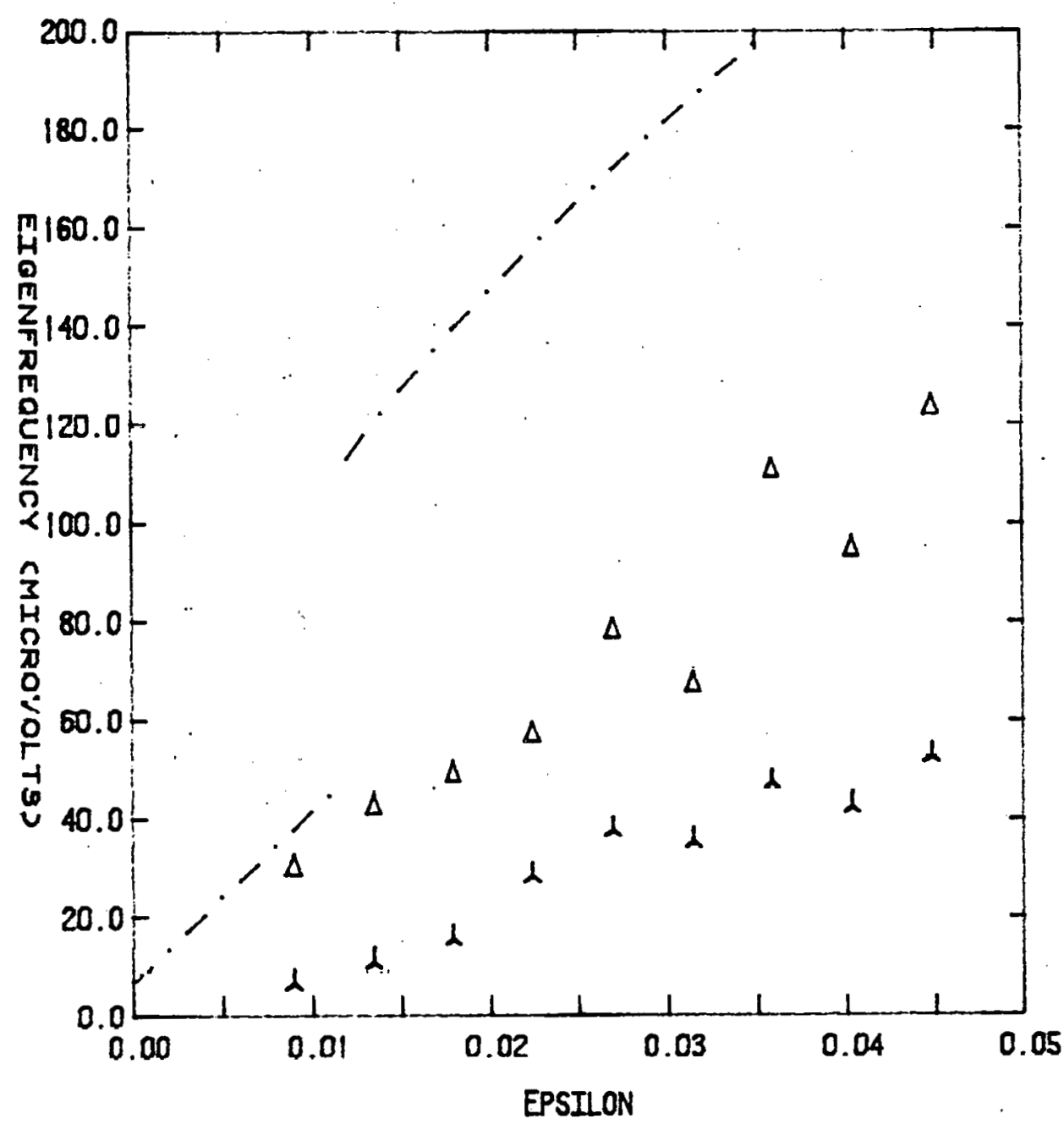

Figure 29. Data for real $(\Delta)$ and imaginary (X) parts of the gap mode for $\# 37$ with $\Gamma=39 \mu \mathrm{V}, \mathrm{Dk}^{2}=14.7 \mu \mathrm{V}$, and $\mathrm{T}_{\mathrm{c}}=1.115 \mathrm{~K}$. OEW theory (dot-dashed) for highfrequency, pure imaginary $\mathrm{L}$-mode. 


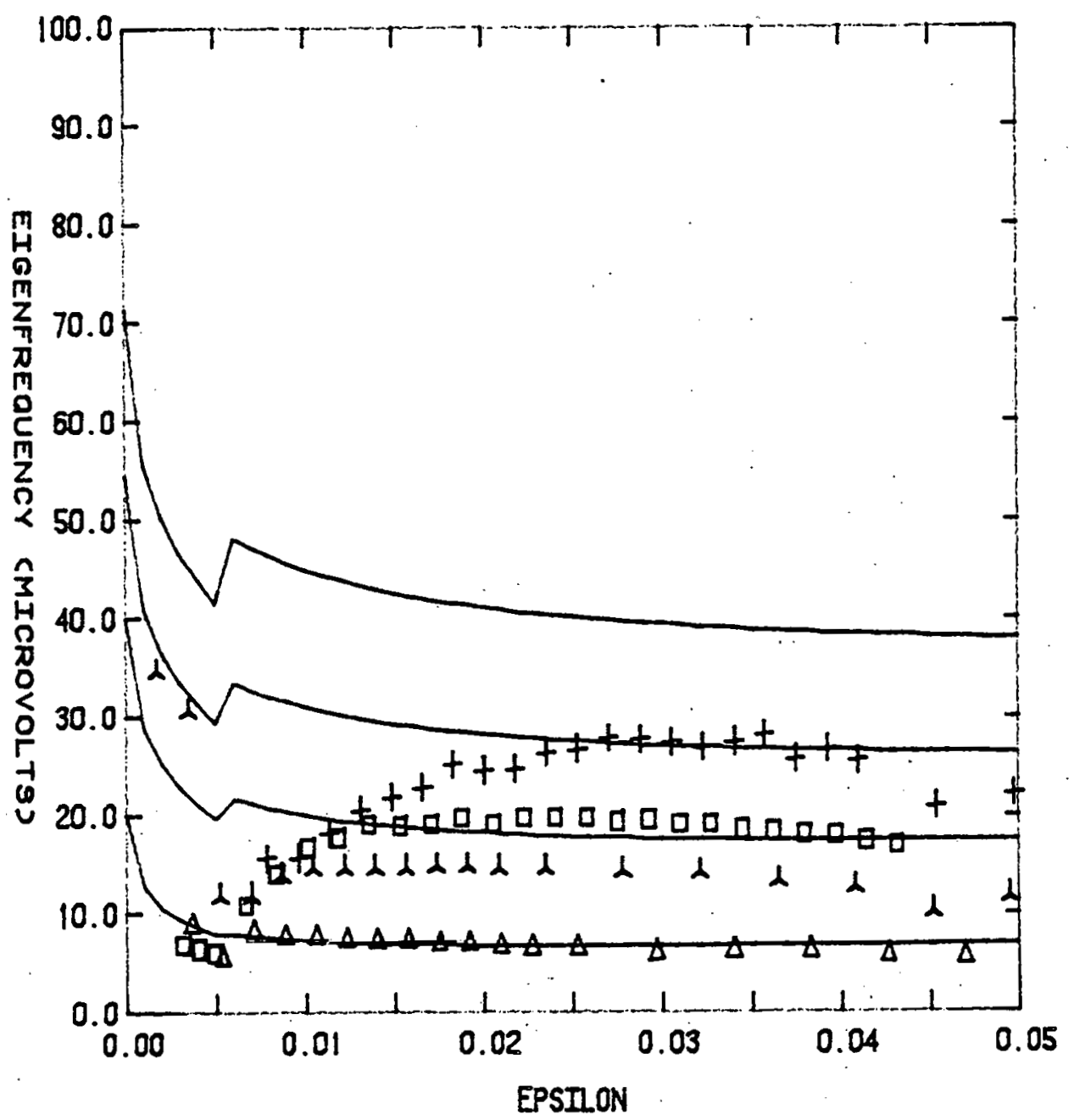

Figure 30. Data for low frequency L-mode for $\# 62$ with $\Gamma=26 \mu \mathrm{V}$ and $: \mathrm{Dk}^{2}=20.2 \mu \mathrm{V}, \mathrm{T}_{\mathrm{c}}=1.155 \mathrm{~K}(\Delta)$; $\mathrm{Dk}^{2}=39.0 \mu \mathrm{V}, \mathrm{T}_{\mathrm{c}}=1.152 \mathrm{~K}(\mathrm{l}) ; \mathrm{Dk}^{2}=54.7 \mu \mathrm{V}$, $\mathrm{T}_{\mathrm{c}}=1.150 \mathrm{~K}(\mathrm{a}) ; \mathrm{Dk}^{2}=71.5 \mu \mathrm{V}, \mathrm{T}_{\mathrm{c}}=1.147 \mathrm{~K}(+)$. Corresponding OEW theory (solid lines) from bottom to top. 


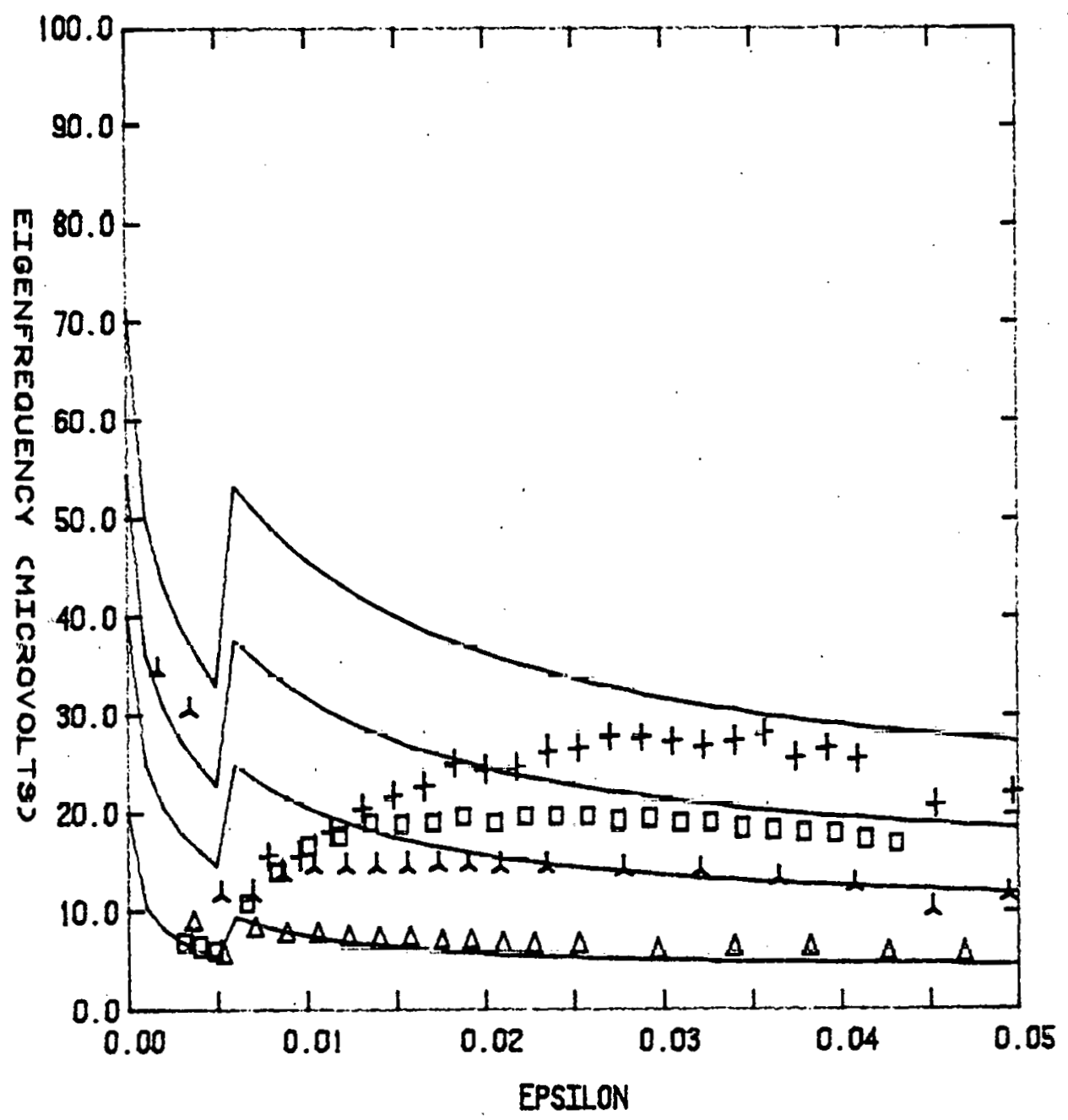

Figure 31. Data for low frequency L-mode for $\$ 62$ with $\Gamma=26 \mu \mathrm{V}$ and: $\mathrm{Dk}^{2}=20.2 \mu \mathrm{V}, \mathrm{T}_{\mathrm{c}}=1.55 \mathrm{~K}(\Delta)$; $\mathrm{Dk}^{2}=39.0 \mu \mathrm{V}, \mathrm{T}_{\mathrm{c}}=1.152 \mathrm{~K}(\alpha) ; \mathrm{Dk}^{2}=54.7 \mu \mathrm{V}$, $\mathrm{T}_{\mathrm{c}}=1.150 \mathrm{~K}(\mathrm{D}) ; \mathrm{Dk}^{2}=71.5 \mu \mathrm{V}, \mathrm{T}_{\mathrm{c}}=1.147 \mathrm{~K}(+)$. Corresponding Dinter theory (solid lines) from bottom to top. 
good agreement with the data for larger values of $\mathrm{Dk}^{2}$ and is especially good for $\varepsilon>0.03$. The discrepancy near $T_{c}$ may be due to the difficulty in fitting in this temperature range where the modes become degenerate.

In Figs. (32) to (37) we plot the low frequency longitudinal mode eigenfrequencies along with the transverse mode eigenfrequencies for samples $\$ 54, \# 37$ and $\$ 45$. Consider for now only the longitudinal mode. Dinter's theory, which is plotted in Figs. (33), (35) and (37), is again in excellent agreement with the experimental data. For comparison the Orbach and Entin-Wohlman theory is superimposed on the same data in Figs. (32), (34) and (36). In general it appears that Dinter's theory is in better agreement with the experimentally determined values of the low-frequency longitudinal mode eigenfrequencies over a wide range of $\mathrm{Dk}^{2}$ (15 to $\left.70 \mu \mathrm{V}\right)$ and of $\Gamma(0$ to $40 \mu \mathrm{V})$.

Each of the three theories considered here agree that the form of the transverse mode eigenfrequency in the gap regime is

$$
i \omega=\omega_{D}+\left(\omega_{D}^{2}-c^{2} k^{2}\right)^{1 / 2}
$$

where the quantities $\omega_{D}$ and $c^{2} k^{2}$ are slightly different for each of the theories. Table III lists the expressions for $\omega_{D}$ and $c^{2} k^{2}$ for each of the theories. The expressions are taken from Eqs. (34), (91), (132) and (133). Only two of the theories derive expressions for the transverse mode in the gapless regime. The solution derived by orbach and Entin-Wohlman is given in Eq. (44), and the Schön-Ambegaokar solution 


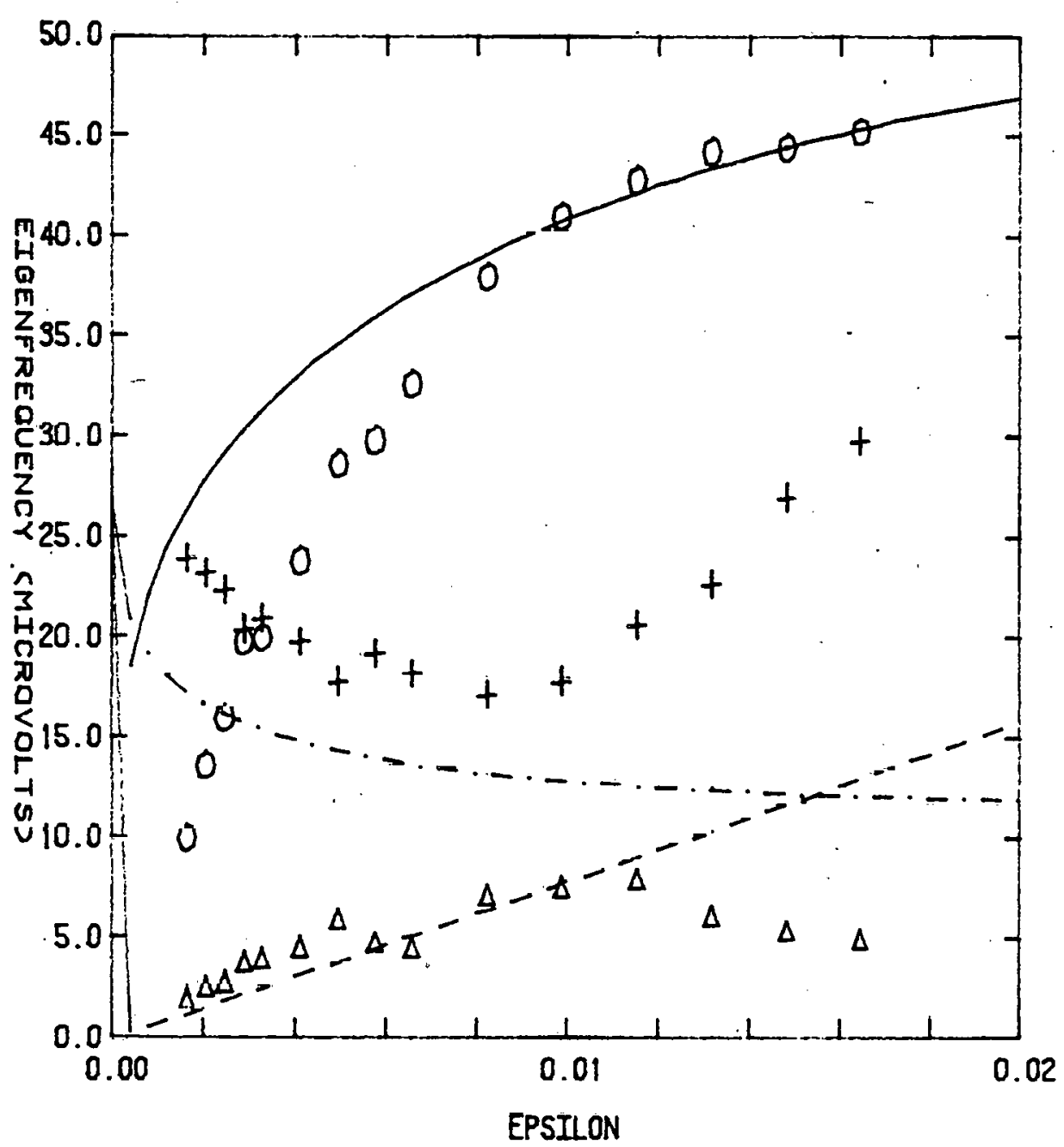

Figure 32. Low frequency $L$-mode ( $\Delta$ and dot-dash), real part of $T$-mode ( 0 and solid) and imaginary part of $\mathrm{T}$-mode (+ and dashed) data and OEW theory for $\$ 54$ with $\Gamma=0, \mathrm{Dk}^{2}=27.0 \mu \mathrm{V}$, and $\mathrm{T}_{\mathrm{c}}=1.214 \mathrm{~K}$. 


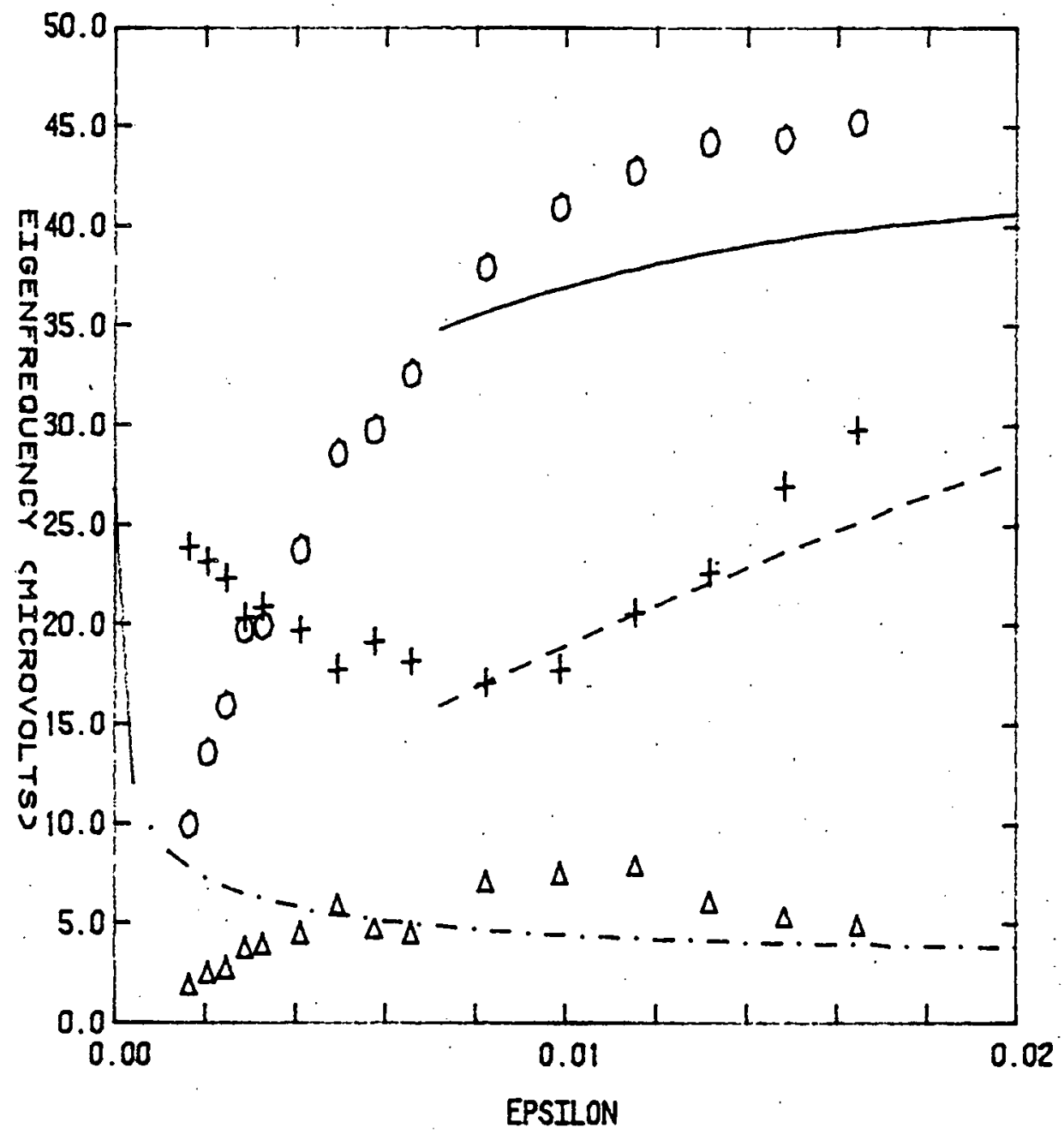

F1gure 33. Low frequency L-mode, ( $\Delta$ and dot-dashed), real part of T-mode ( 0 and soli, $)$ and imaginary part of T-mode ( + and dashed) data and Dinter theory for $\$$ 非 with $\Gamma=0, \mathrm{Dk}^{2}=27.0 \mu \mathrm{V}$, and $\mathrm{T}_{\mathrm{c}}=1.214 \mathrm{~K}$. 


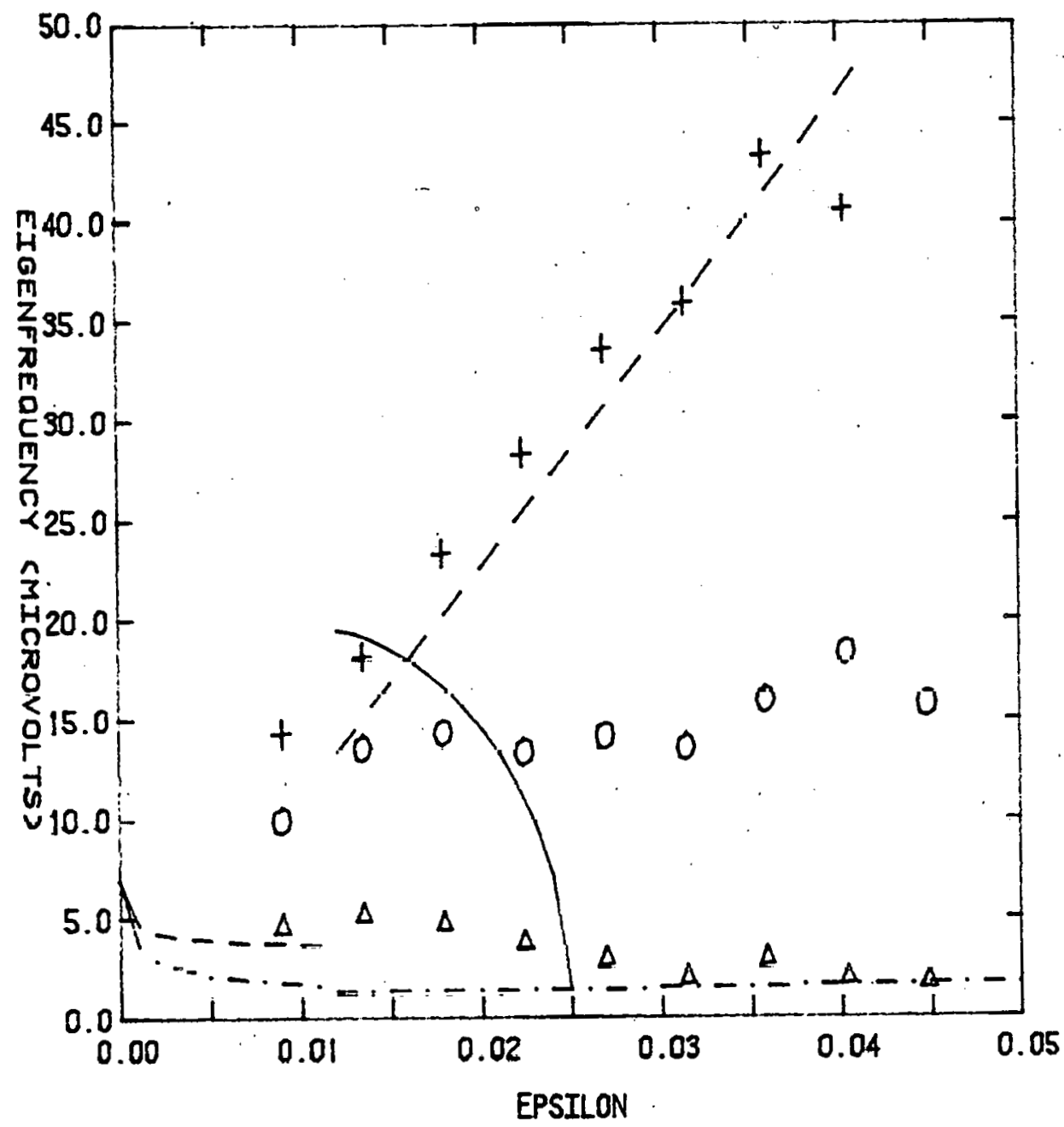

Figure 34. Low frequency L-mode ( $\Delta$ and dot-dashed), real part of $T$-mode ( 0 and solid) and imaginary part of T-mode (+ and dashed) data and OEW theory for $\$ 37$ with $\Gamma=39 \mu \mathrm{V}, \mathrm{Dk}^{2}=14.7 \mu \mathrm{V}$, and $\mathrm{T}_{\mathrm{c}}=1.115 \mathrm{~K}$. 


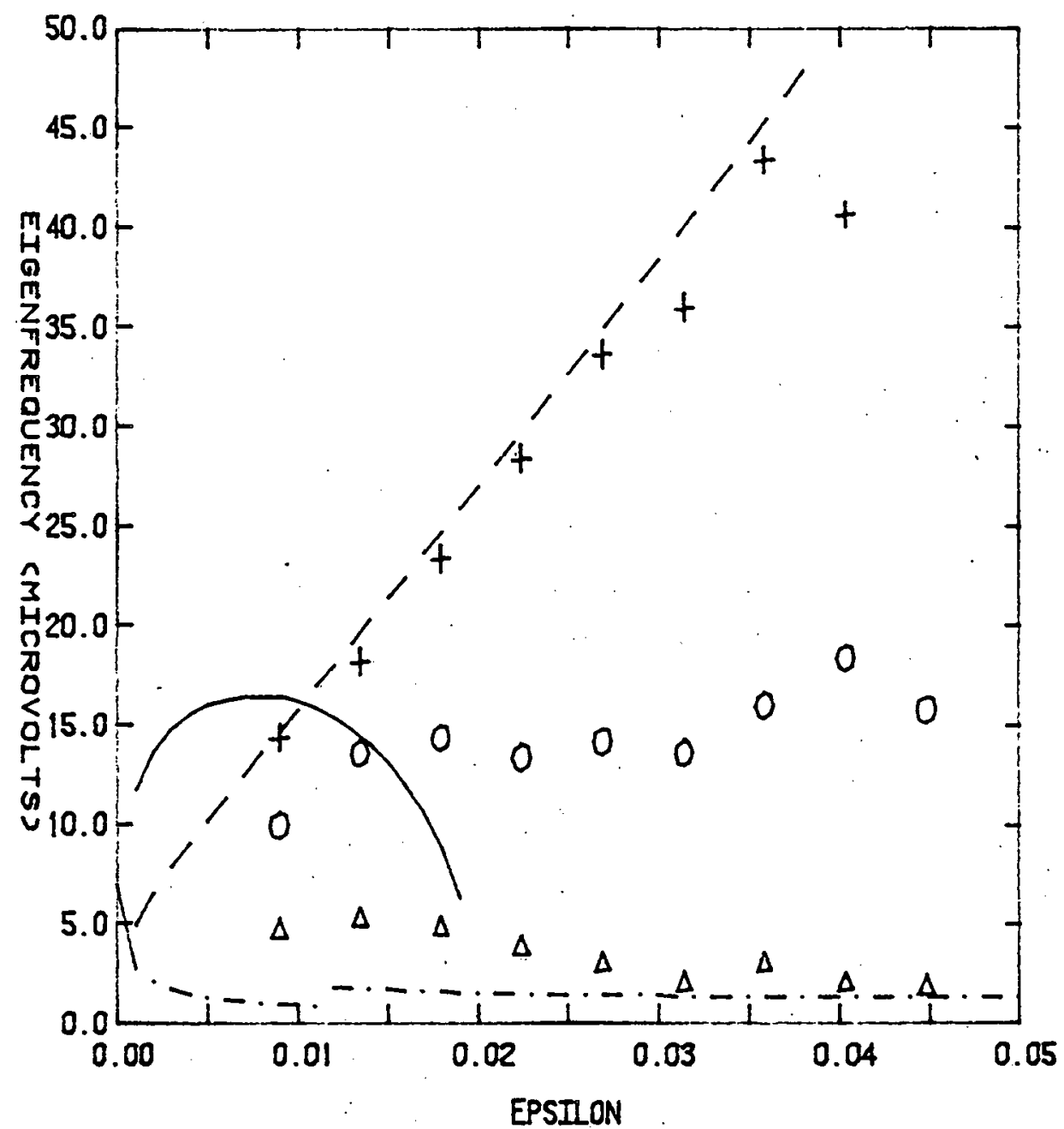

Figure 35. Low frequency L-mode ( $\Delta$ and dot-dashed), real part $(O$ and solid) and imaginary part (th and dashed) of T-mode data and Dinter theory for $\$ 37$ with $\Gamma=39 \mu \mathrm{V}$, $\mathrm{Dk}^{2}=14.7 \mu \mathrm{V}$, and $\mathrm{T}_{\mathrm{c}}=1.115 \mathrm{~K}$. 


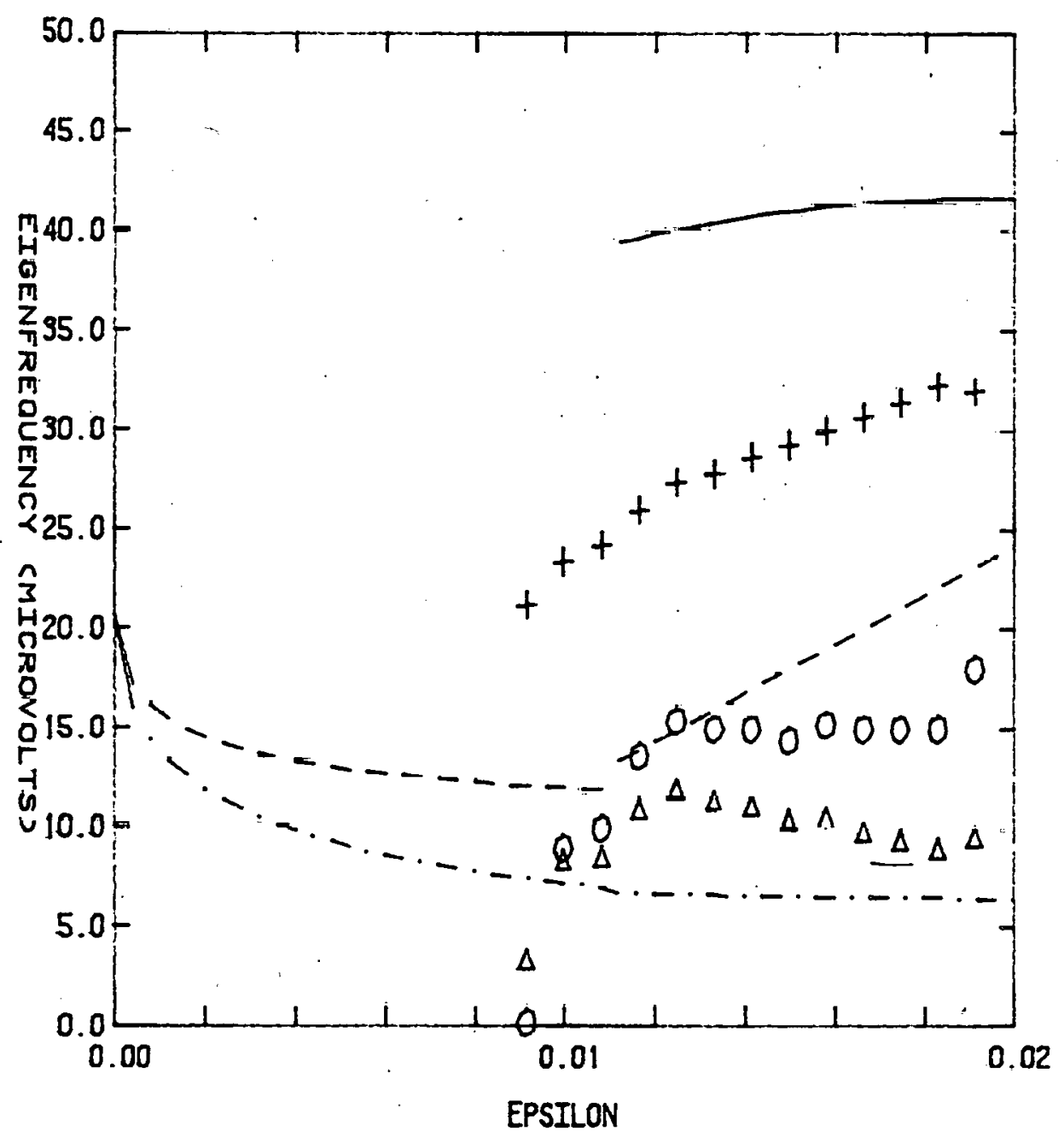

Figure 36. Low frequency L-mode ( $\Delta$ and dot-dashed), real part ( 0 and solid) and imaginary part (t and dashed) of $T$-mode data and OEW theory for $\$ 45$ with $\Gamma=41 \mu \mathrm{V}$, $\mathrm{Dk}^{2}=20.6 \mu \mathrm{V}$ and $\mathrm{T}_{\mathrm{c}}=1.203 \mathrm{~K}$. 


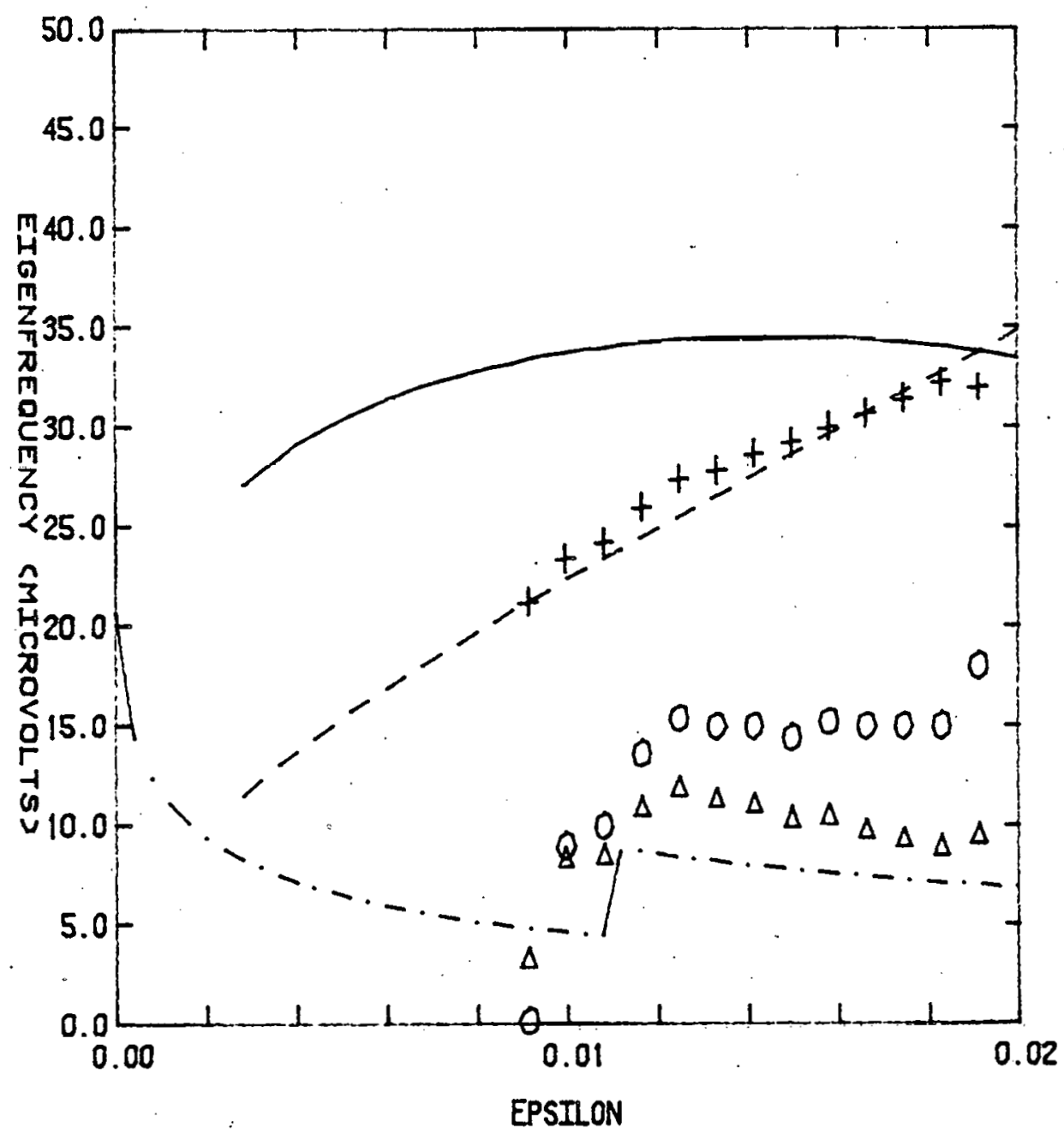

Figure 37. Low frequency L-mode ( $\Delta$ and dot-dashed), real part ( 0 and solid) and imaginary part (t and dashed) of $T$-mode data and Dinter theory for $\$$ 非 with $\Gamma=41 \mu \mathrm{V}$, $\mathrm{Dk}^{2}=20.6 \mu \mathrm{V}$ and $\mathrm{T}_{\mathrm{c}}=1.203 \mathrm{~K}$. 
TABLE III

Expressions for the Real and Imaginary Part of the Transverse-Mode Eigenfrequency in the Gap Regime Given by $i \omega=\omega_{D}+\left(\omega_{D}^{2}-c^{2} \cdot k^{2}\right)^{1 / 2}$ for Each of Three Theories

\begin{tabular}{|c|c|c|c|}
\hline Theory & $\omega_{D}$ & $c^{2} k^{2}$ & Constraints \\
\hline $\begin{array}{l}\text { Orbach and } \\
\text { Entin-Wohlman }\end{array}$ & $\frac{\pi \Delta^{2}}{4 \mathrm{~T}} \frac{\psi^{\prime}(1 / 2+\rho)}{\psi^{\prime}(1 / 2)}$ & $2 \Delta \mathrm{Dk}^{2} \frac{\psi^{\prime}(1 / 2+\rho)}{\psi^{\prime}(1 / 2)}$ & $\Delta \gg \Gamma$ \\
\hline Dinter & $\frac{\pi \Delta^{2}}{4 \mathrm{~T}}+\frac{\mathrm{Dk}^{2}}{\pi} \ln \frac{2 \Delta}{\omega}$ & $2 \Delta \mathrm{Dk}^{2}$ & $\mathrm{Dk}^{2} \ll \omega \ll 2 \Delta$ \\
\hline $\begin{array}{l}\text { Schön and } \\
\text { Añilegaokar }\end{array}$ & $\frac{\pi \Delta^{2}}{4 \mathrm{~T}}+\sim \mathrm{Dk}{ }^{2}+\sim \frac{\Gamma}{2}$ & $\begin{array}{c}2 \Delta \mathrm{Dk}^{2} \mathrm{x} \\
{\left[1+\frac{\pi \Delta}{4 \mathrm{~T}}\left(1+U\left(\ln \frac{\Lambda}{\omega}\right)\right)\right]}\end{array}$ & $\Delta \gg \Gamma$ \\
\hline
\end{tabular}


is given in Eq. (135). Both solutions are diffusive in nature and are valid for $\Gamma$. > $\Delta$ only. Although the predictions are substantially different, the difficulties we encounter in extracting the eigenfrequencies from the data near $T_{c}$ do not allow us to confirm or reject either theory.

In Fig. (32) to (52) we display the experimental eigenfrequencies for the transverse mode. Each theory is superimposed over its range of validity. For Dinter's theory we choose to plot only in the range $\Delta>\mathrm{Dk}^{2}$ to satisfy the constraint given in Table III. The damping is accounted for extremely well by the theory of Dinter. The theories of Schön and Ambegaokar and of Orbach and EntinWohlman predict the same $\pi \Delta^{2} / 4 \mathrm{~T}$ temperature-dependence as Dinter's theory but each admits to a $\mathrm{Dk}^{2}$ correction which is not precisely defined. The term $\mathrm{Dk}^{2} \ln (2 \Delta / \omega) / \pi$ which is contained in Dinter's theory depends weakly on $\omega$. We have inserted $\omega=\mathrm{Dk}^{2}+\pi \Delta^{2} / 4 \mathrm{~T}$, , where this term appears in Dinter's expression for the damping. For our experimental parameters, the exact solution is not noticeably different from the damping calculated in this manner.

The real part of the transverse-mode eigenfrequency for each theory is in qualitative agreement with theory. Each of the theories is plotted to $\varepsilon=0.05$ which corresponds to $\Delta / T \simeq 0.7$. It is expected that higher order terms in $\Delta / T$ are important near this limit.

Each of the theories predicts a temperature "windcw" of propagation in the gap regime in which the real part of the eigenfrequency exceeds the 1maginary part. It should be noted that the 


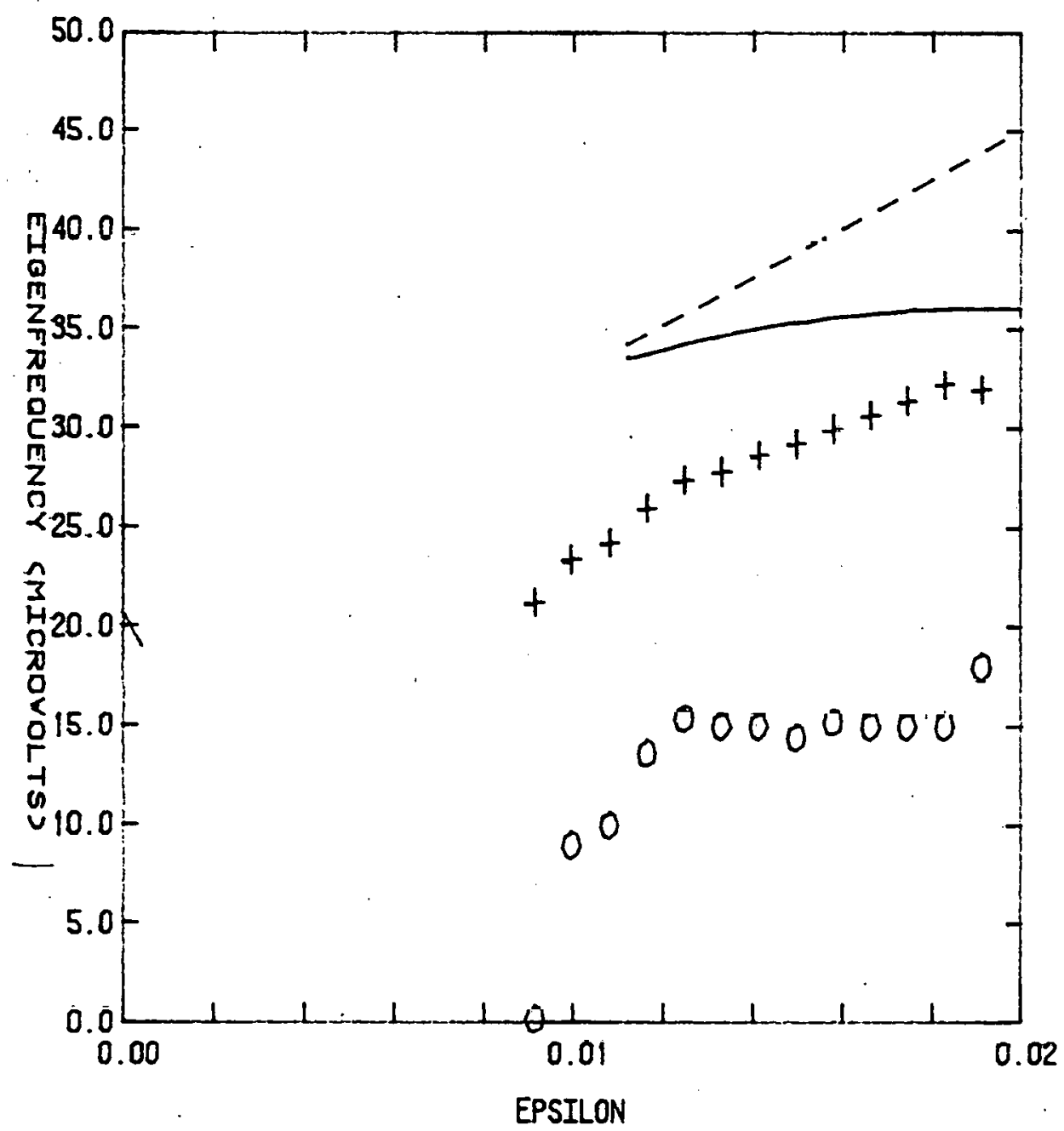

Figure 38: Real part ( $O$ and solid) and imaginary part ( + and dashed) of T-mode data and SA theory for $\$$ " 45 with $\Gamma=41 \mu \mathrm{V}, \mathrm{Dk}^{2}=21 \mu \mathrm{V}, \mathrm{T}_{\mathrm{c}}=1.214 \mathrm{k}$. 


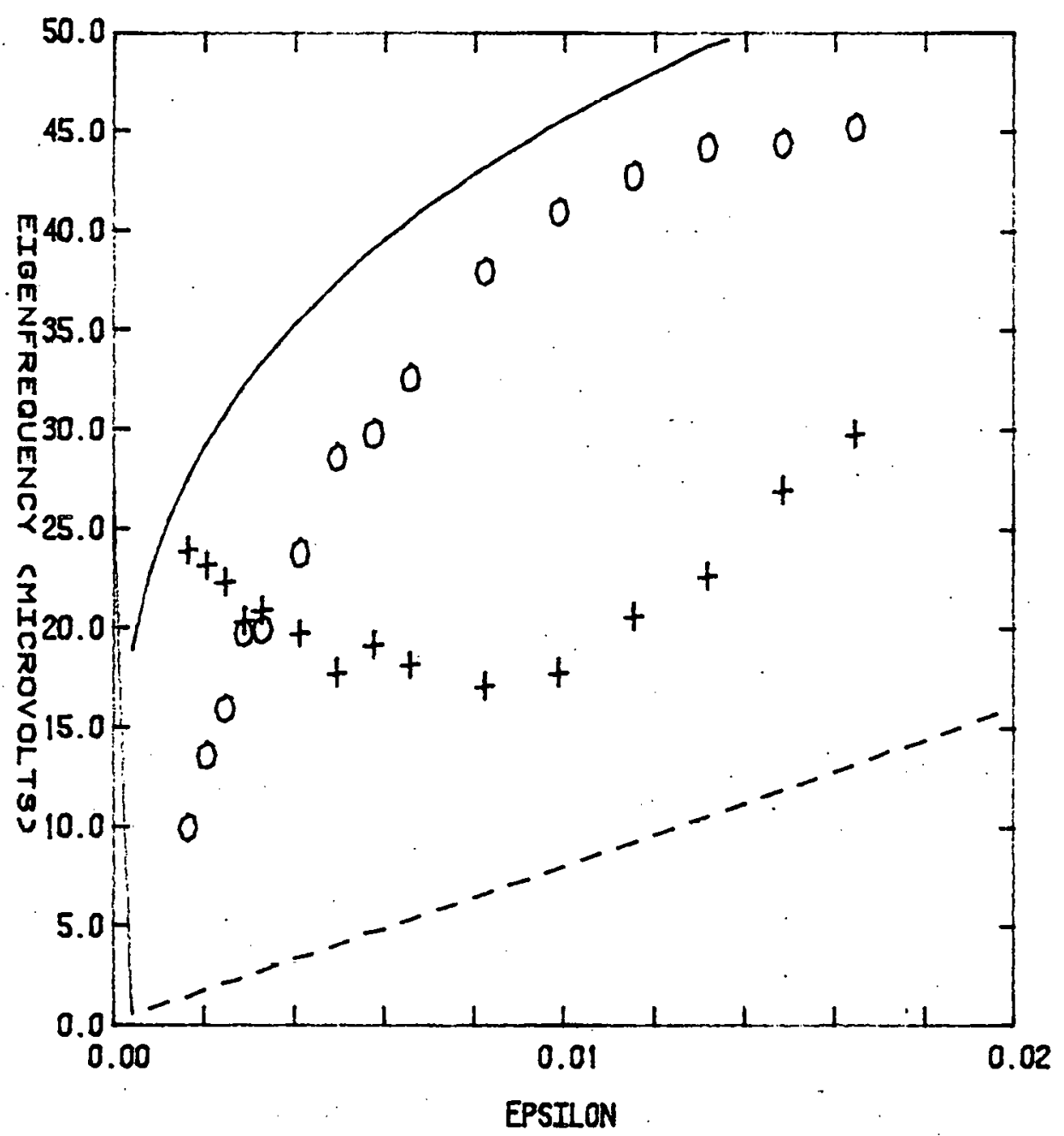

Figure 39. Real part ( 0 and solid) and imaginary part (t and dashed) of $\mathrm{T}$-mode data and SA theory for $\$$ 非 4 with $\Gamma=0, \mathrm{Dk}^{2}=27 \mu \mathrm{V}, \mathrm{T}_{\mathrm{C}}=1.214 \mathrm{~K}$. 


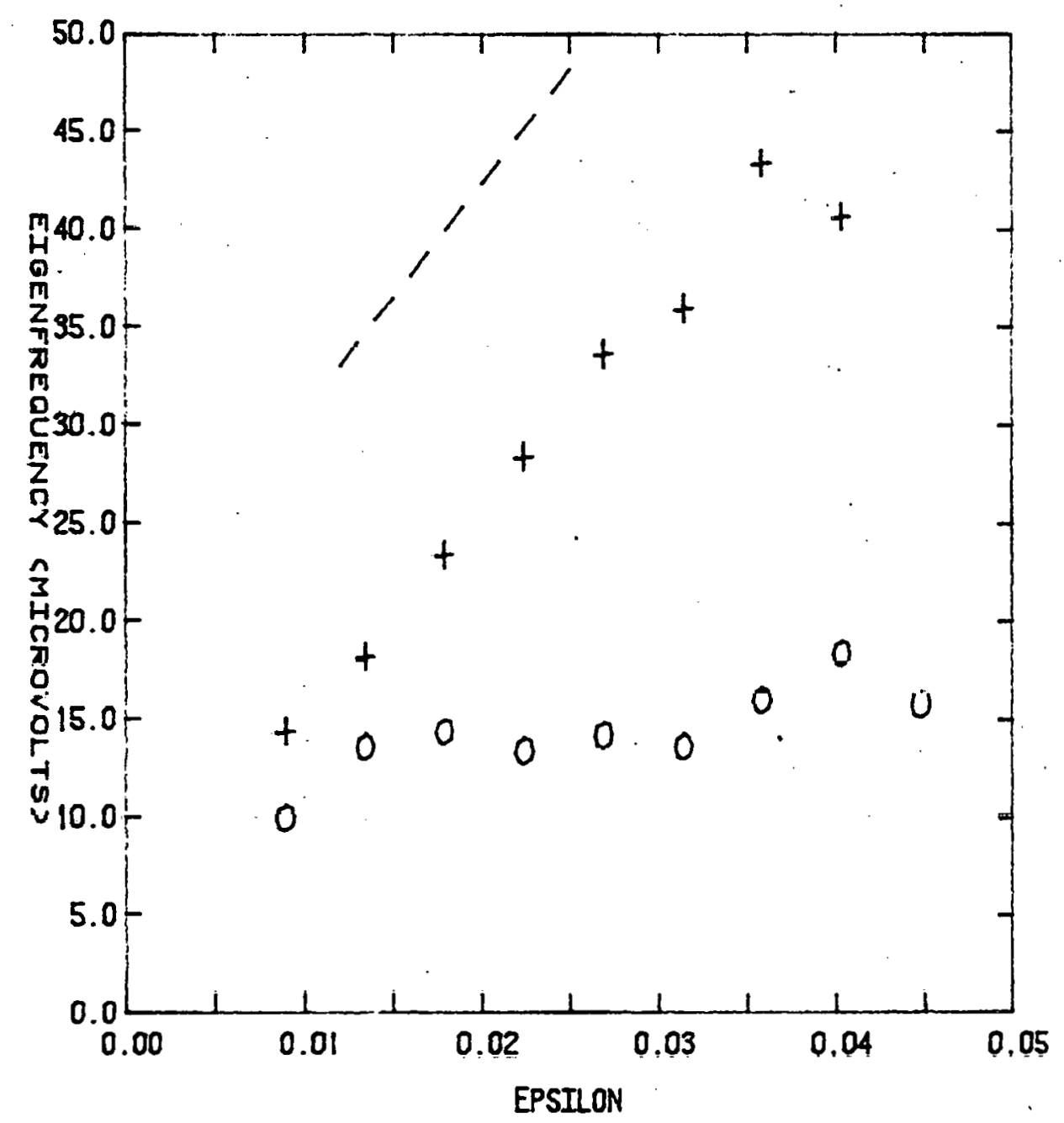

Figure 40. Real part ( 0 and solid) and 1maglnary part (t and dashed) of $T$-mode data and SA theory for $\$ 37$ with $\Gamma=39 \mu \mathrm{V}, \mathrm{Dk}^{2}=14.7 \mu \mathrm{V}$ and $\mathrm{T}_{\mathrm{C}}=1.115 \mathrm{~K}$. 


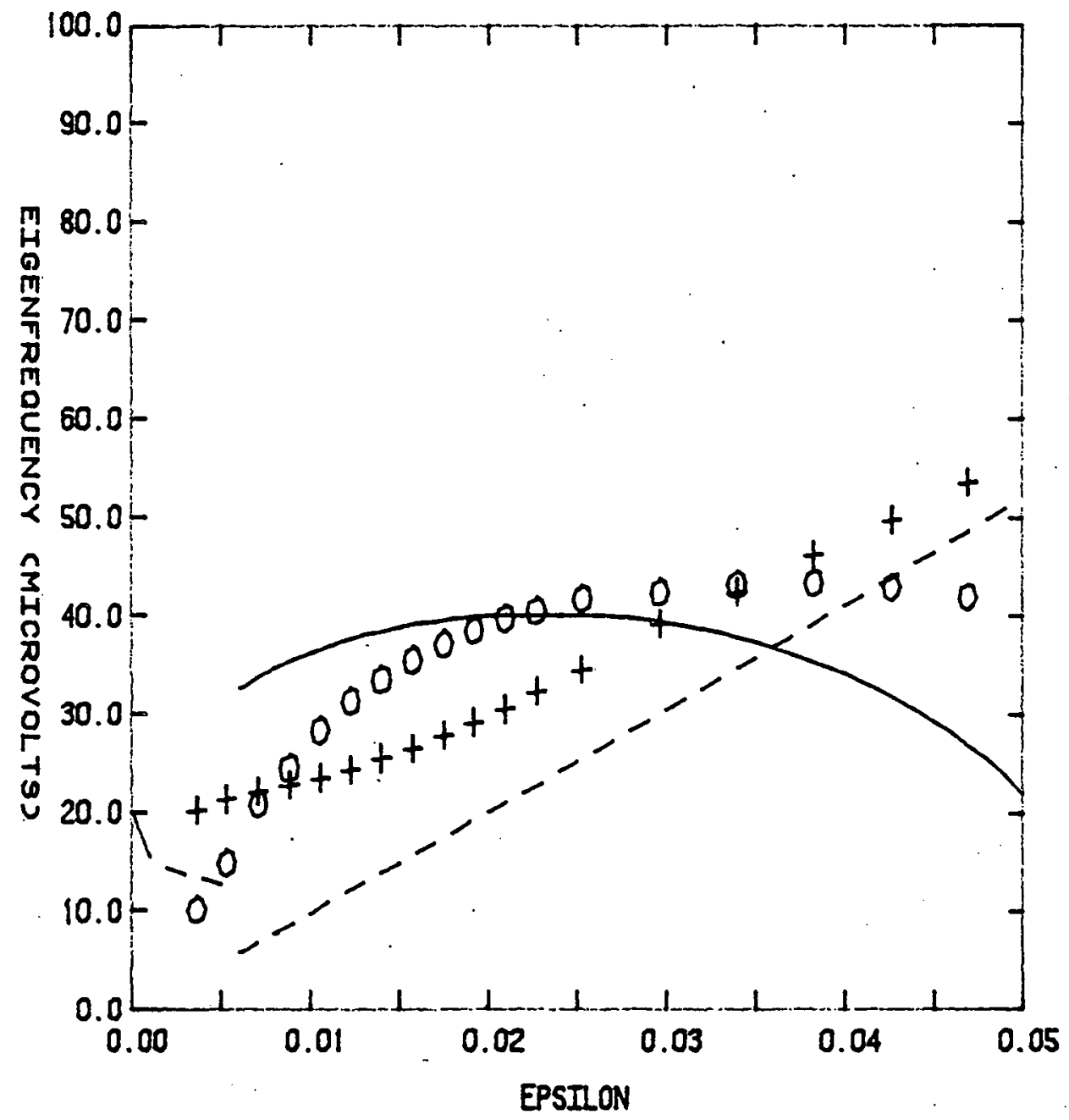

Figure 41. Real part (0 and solid) and imaginary part (t and dashed) of $T$-mode data and OEW theory for $\$ 62$ with $\Gamma=26 \mu \mathrm{V}, \mathrm{Dk}^{2}=20.2 \mu \mathrm{V}$, and $\mathrm{T}_{\mathrm{C}}=1.155 \mathrm{~K}$. 


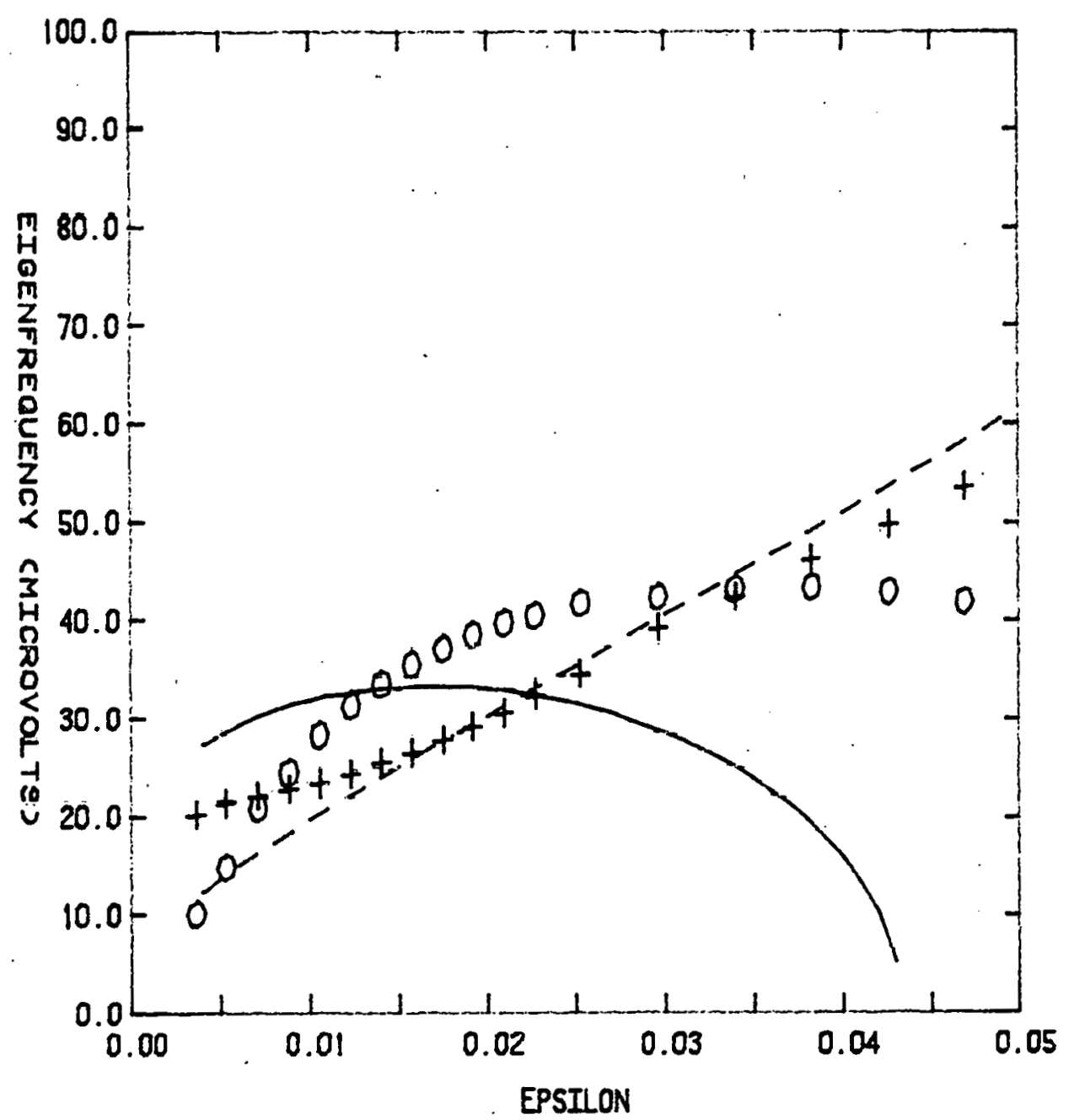

Figure 42. Real part ( 0 and solid) and imaginary part (+ and dashed) of T-mode data and Dinter theory for $\$ 62$ with $\Gamma=26 \mu \mathrm{V}, \mathrm{Dk}^{2}=20.2 \mu \mathrm{V}$ and $\mathrm{T}_{\mathrm{c}}=1.155 \mathrm{~K}$. 


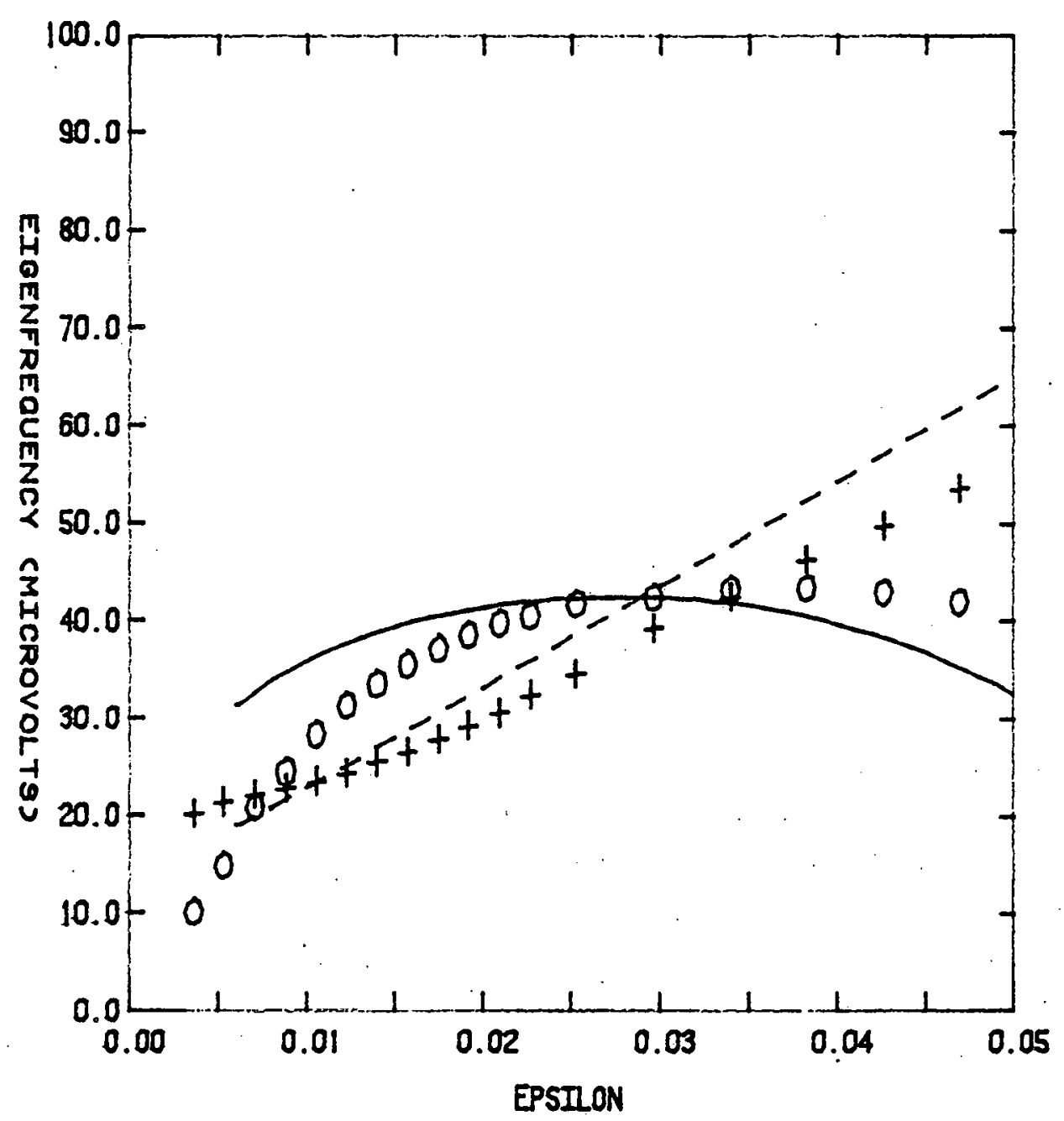

Figure 43. Real part ( 0 and solid) and imaginary part (+ and dashed) of T-mode data and SA theory for $\# 62$ with $\Gamma=26 \mu \mathrm{V}, \mathrm{Dk}^{2}=20.2 \mu \mathrm{V}$ and $\mathrm{T}_{\mathrm{c}}=1.155 \mathrm{~K}$. 


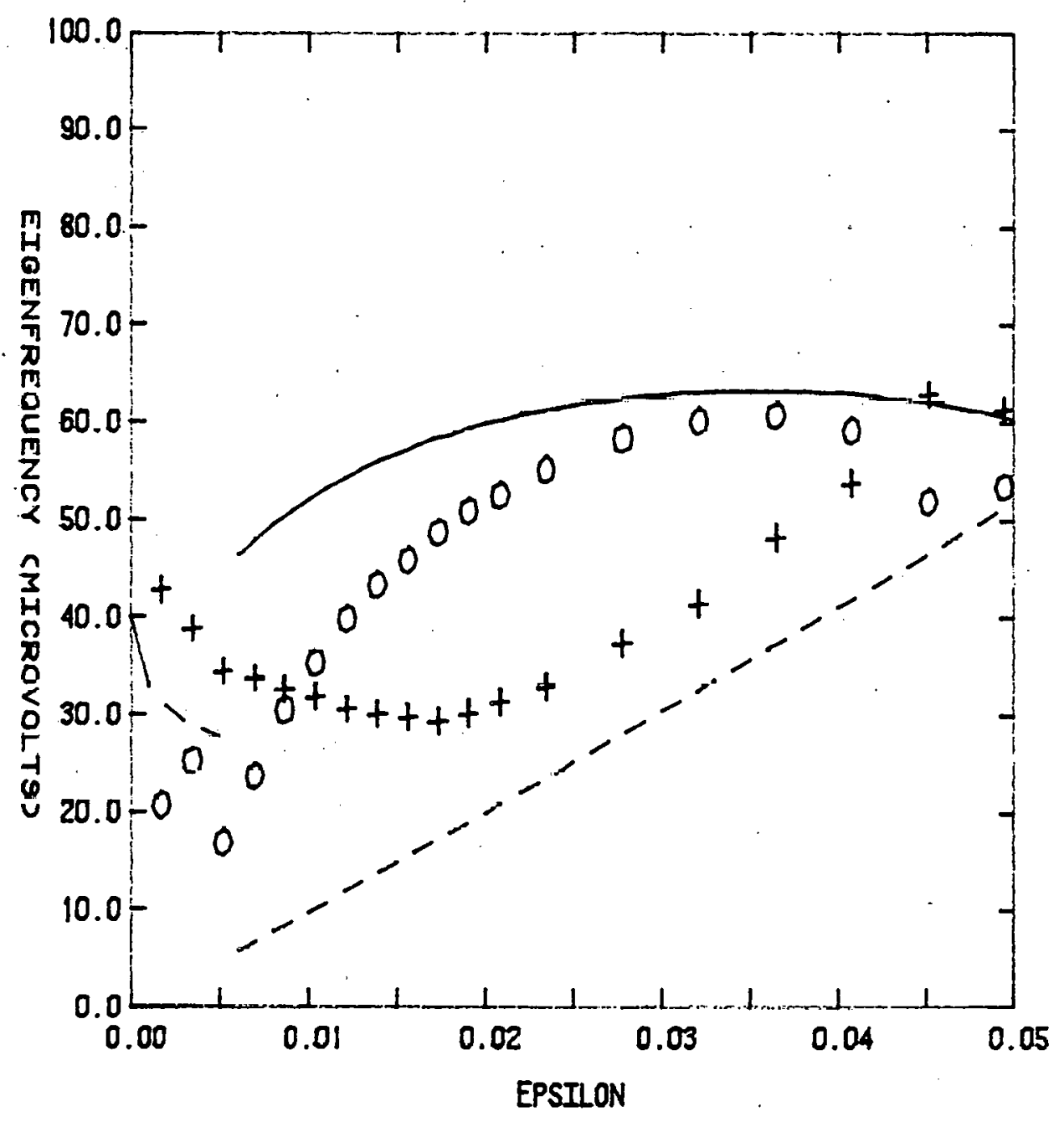

Figure 44. Real part ( 0 and sol1d) and 1maginary part (t and dashed) of $\mathrm{T}$-mode data and OEW theory for $\# 62$ with $\Gamma=26 \mu \mathrm{V}, \mathrm{Dk}^{2}=20.2 \mu \mathrm{V}$ and $\mathrm{T}_{\mathrm{c}}=1.155 \mathrm{~K}$. 


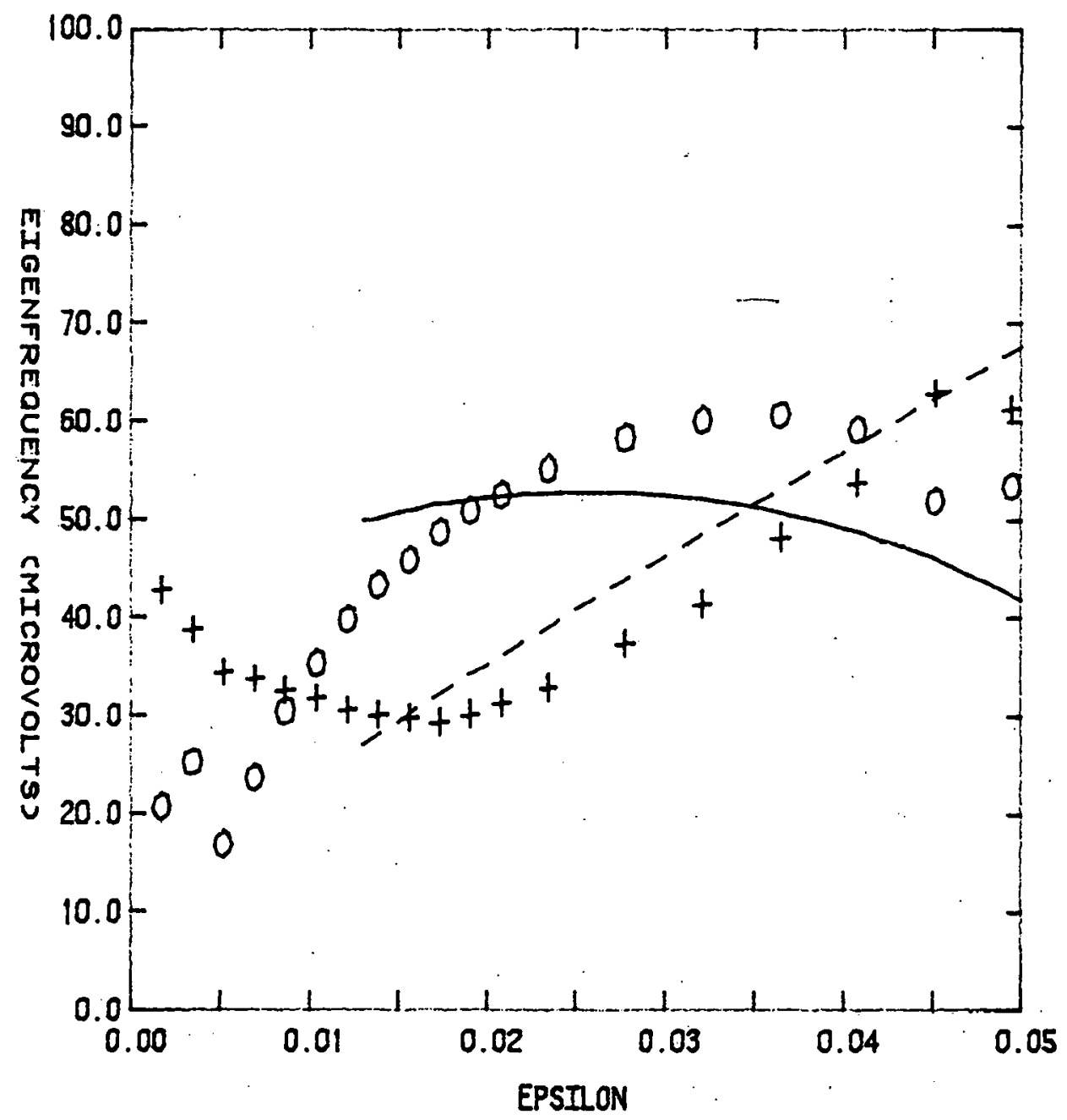

Figure 45. Real part ( 0 and solid) and imaginary part (t and dashed) of T-mode data and Dinter theory for $\$ 62$ with $\Gamma=26 \mu \mathrm{V}, \mathrm{Dk}^{2}=39.0 \mu \mathrm{V}$ and $\mathrm{T}_{\mathrm{c}}=1.152 \mathrm{~K}$. 


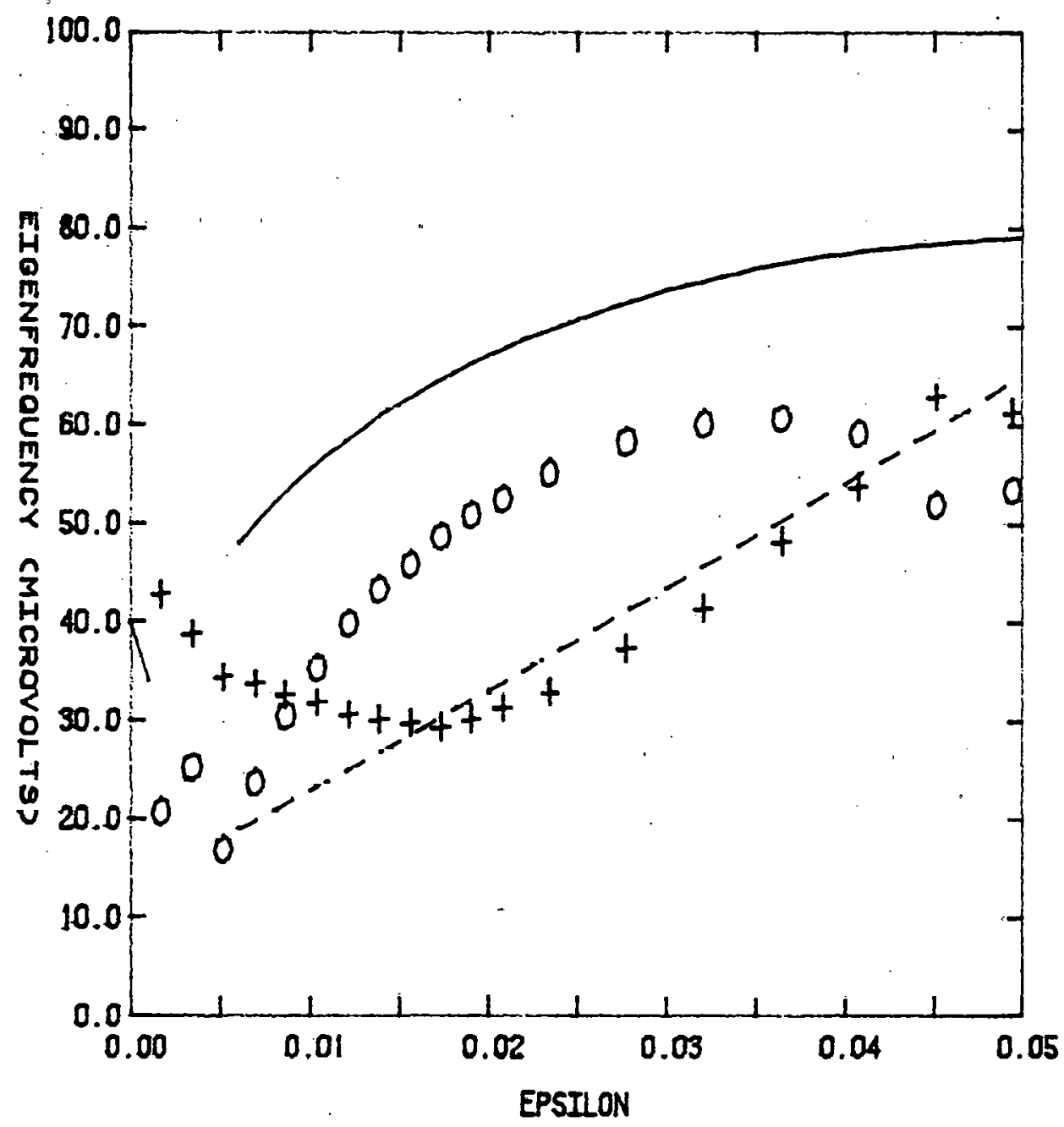

Figure 46. Real part ( 0 and solid) and imaginary part ( $t$ and dashed) of T-mode data and SA theory for $\$$ 作 62 with $\Gamma=26 \mu \mathrm{V}, \mathrm{Dk}^{2}=39.0 \mu \mathrm{V}$ and $\mathrm{T}_{\mathrm{c}}=1.152 \mathrm{k}$. 


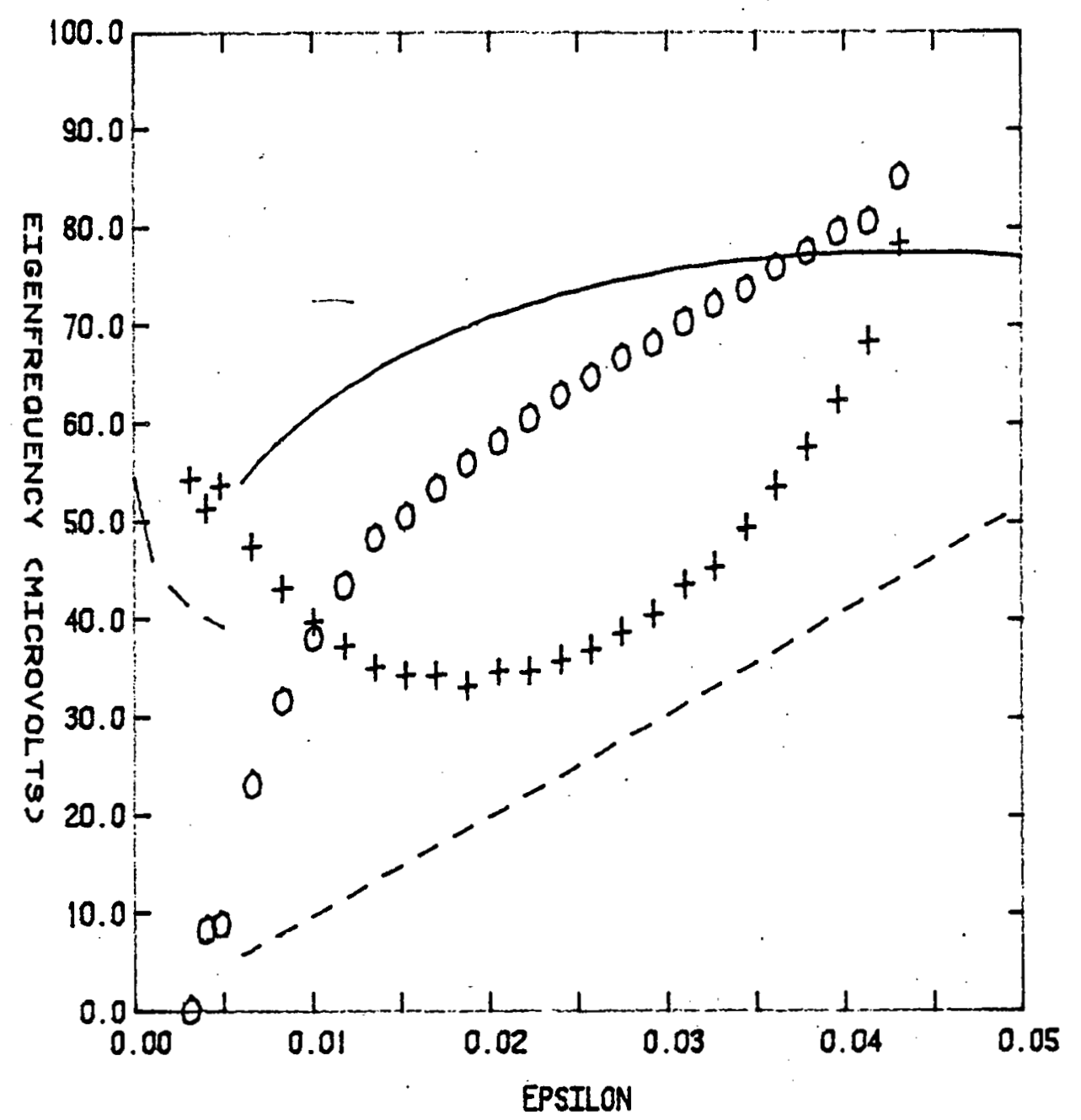

Figure 47. Real part ( 0 and solid) and imaginary part ( + and dashed) of $\mathrm{T}$-mode data and OEW theory for $\$ 62$ with $\Gamma=26 \mu \mathrm{V}, \mathrm{Dk}^{2}=54.7 \mu \mathrm{V}$, and $\mathrm{T}_{\mathrm{c}}=1.150 \mathrm{~K}$. 


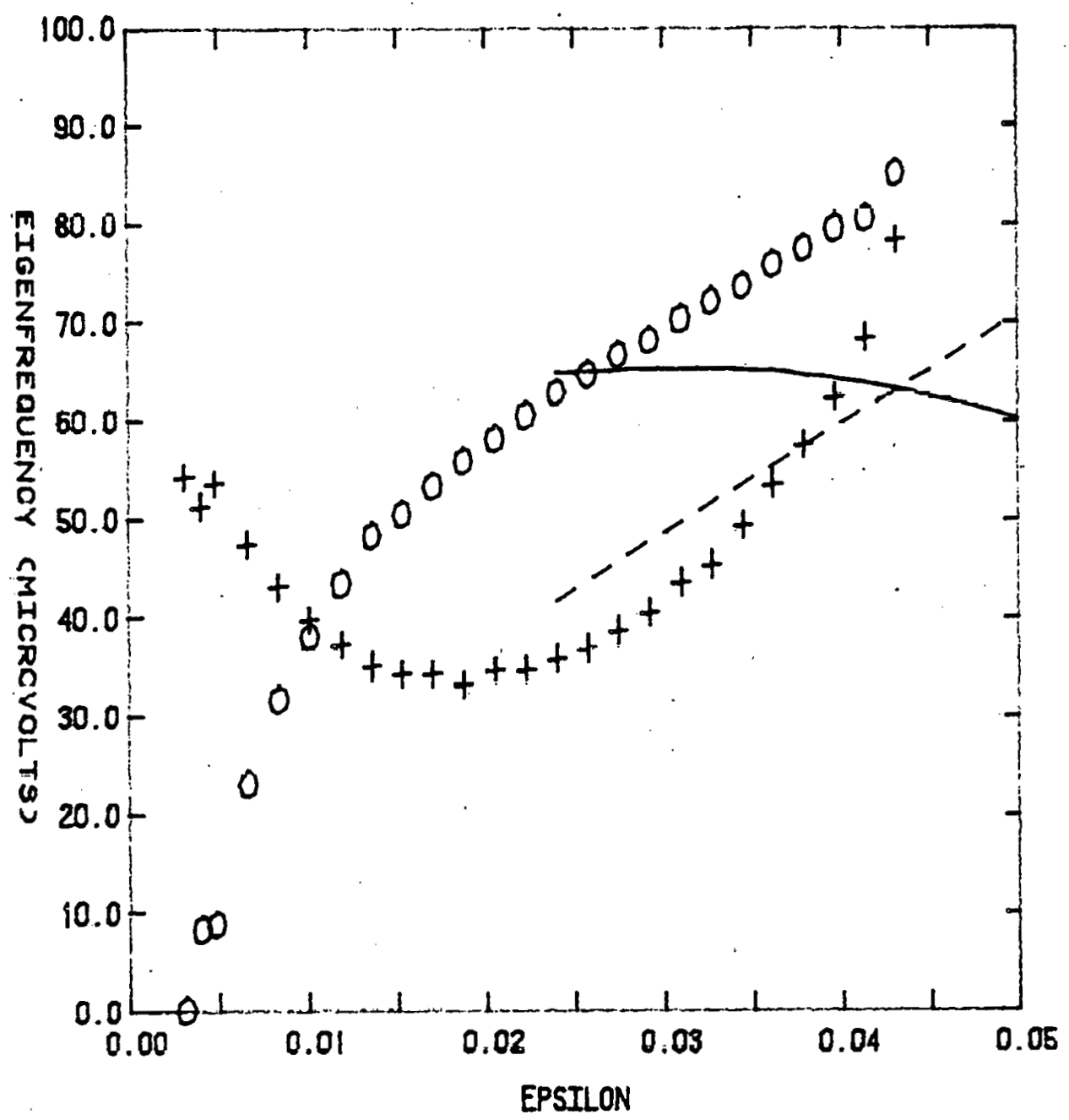

Flgure 48. Real part ( 0 and solid) and imaginary part (t and dashed) of $T$-mode data and Dinter theory for $\$$ \#62 with $\Gamma=26 \mu \mathrm{V}, \mathrm{Dk}^{2}=54.7 \mu \mathrm{V}$, and $\mathrm{T}_{\mathrm{c}}=1.150 \mathrm{~K}$. 


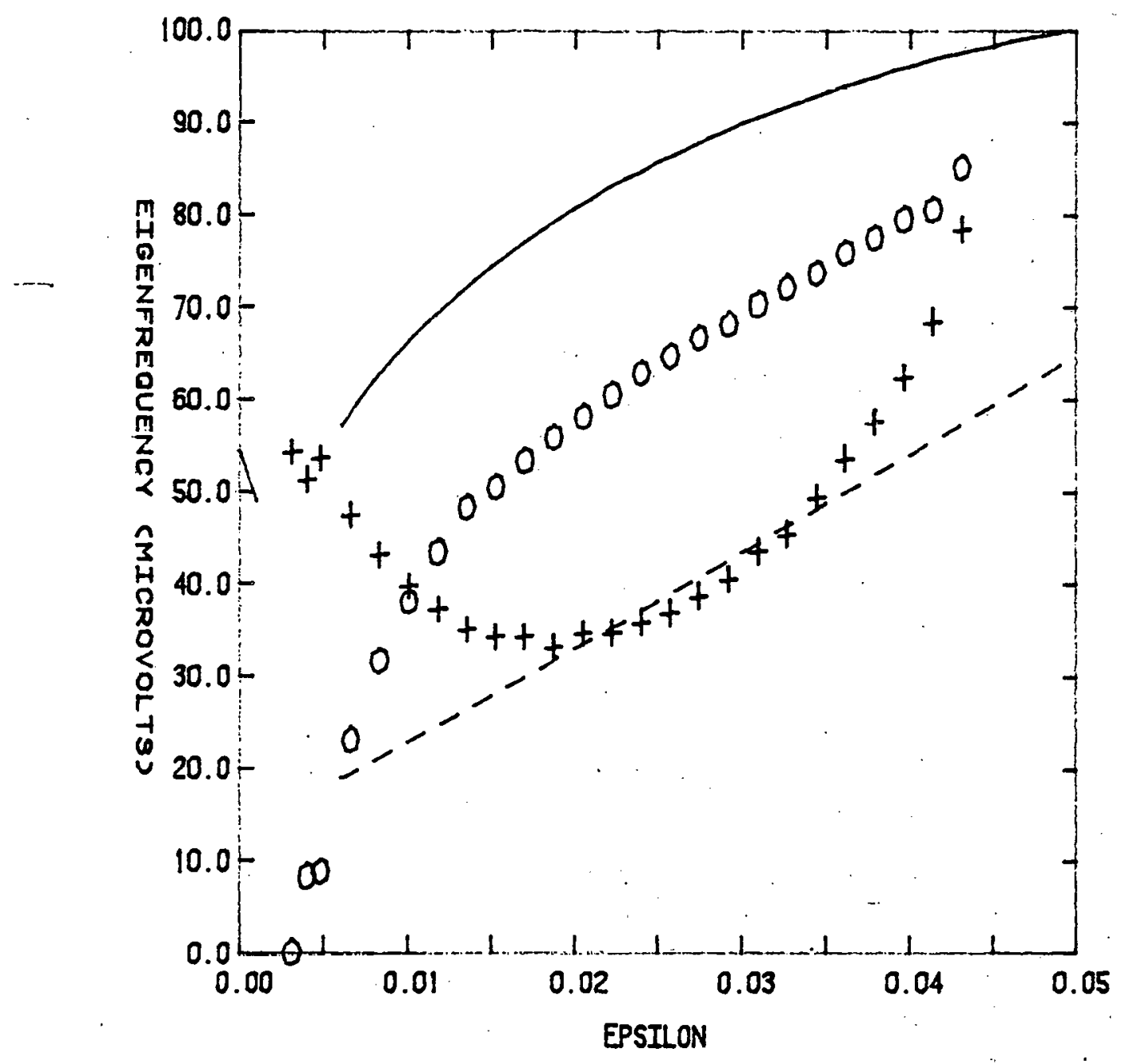

Figure 49. Real part (0 and solid) and imaginary part (t and dashed) of $\mathrm{T}$-mode data and SA theory for $\$ 62$ with $\Gamma=26 \mu \mathrm{V}, \mathrm{Dk}^{2}=54.7 \mu \mathrm{V}$, and $\mathrm{T}_{\mathrm{c}}=1.150 \mathrm{~K}$. 


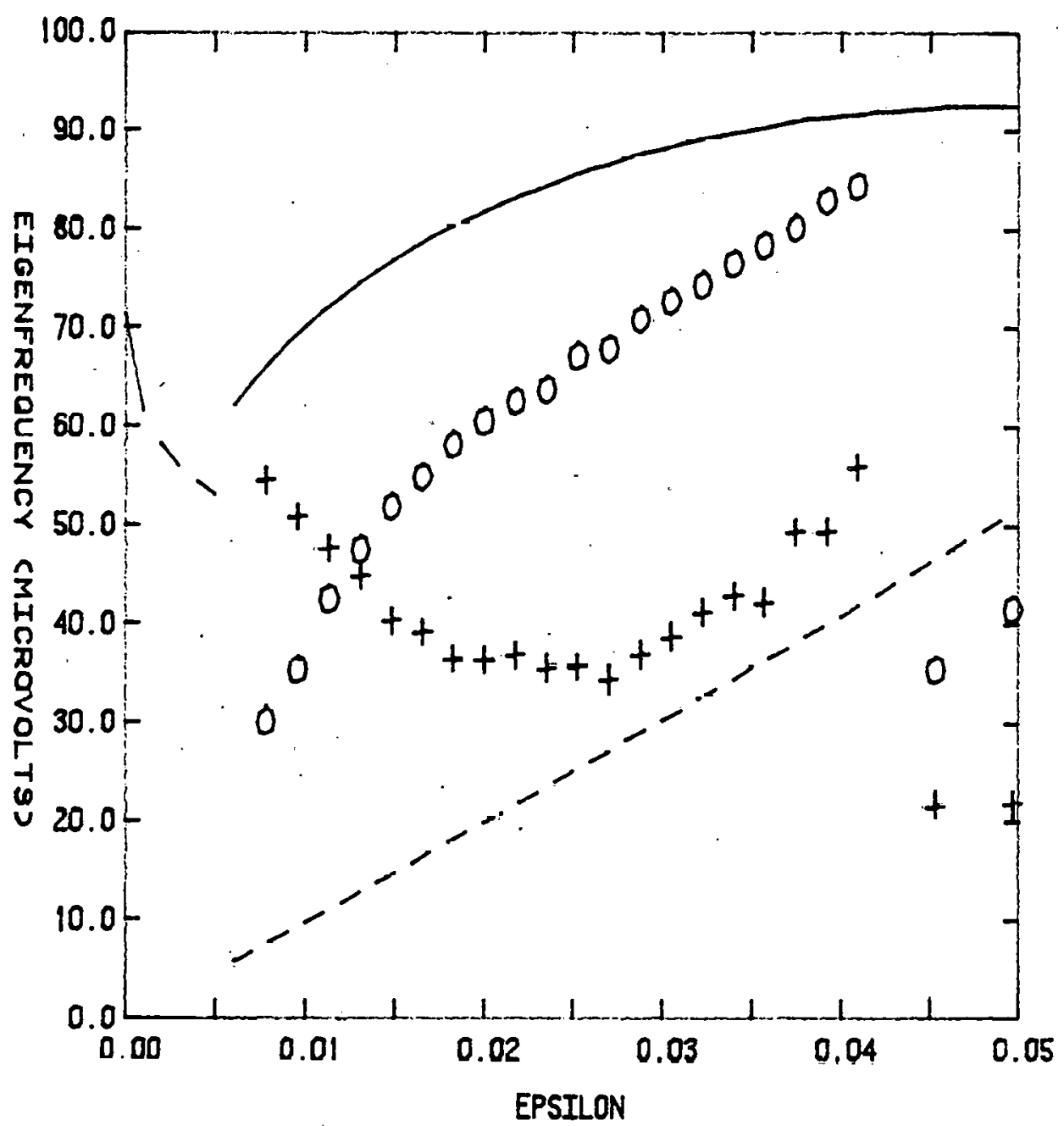

Figure 50. Real part ( 0 and solid) and imaginary part (+ and

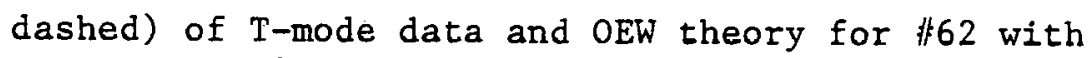
$\Gamma=261 . \mathrm{V}, \mathrm{Dk}^{2}=71,51 . \mathrm{V}$, and $T_{\mathrm{c}}=1.147 \mathrm{~K}$. 


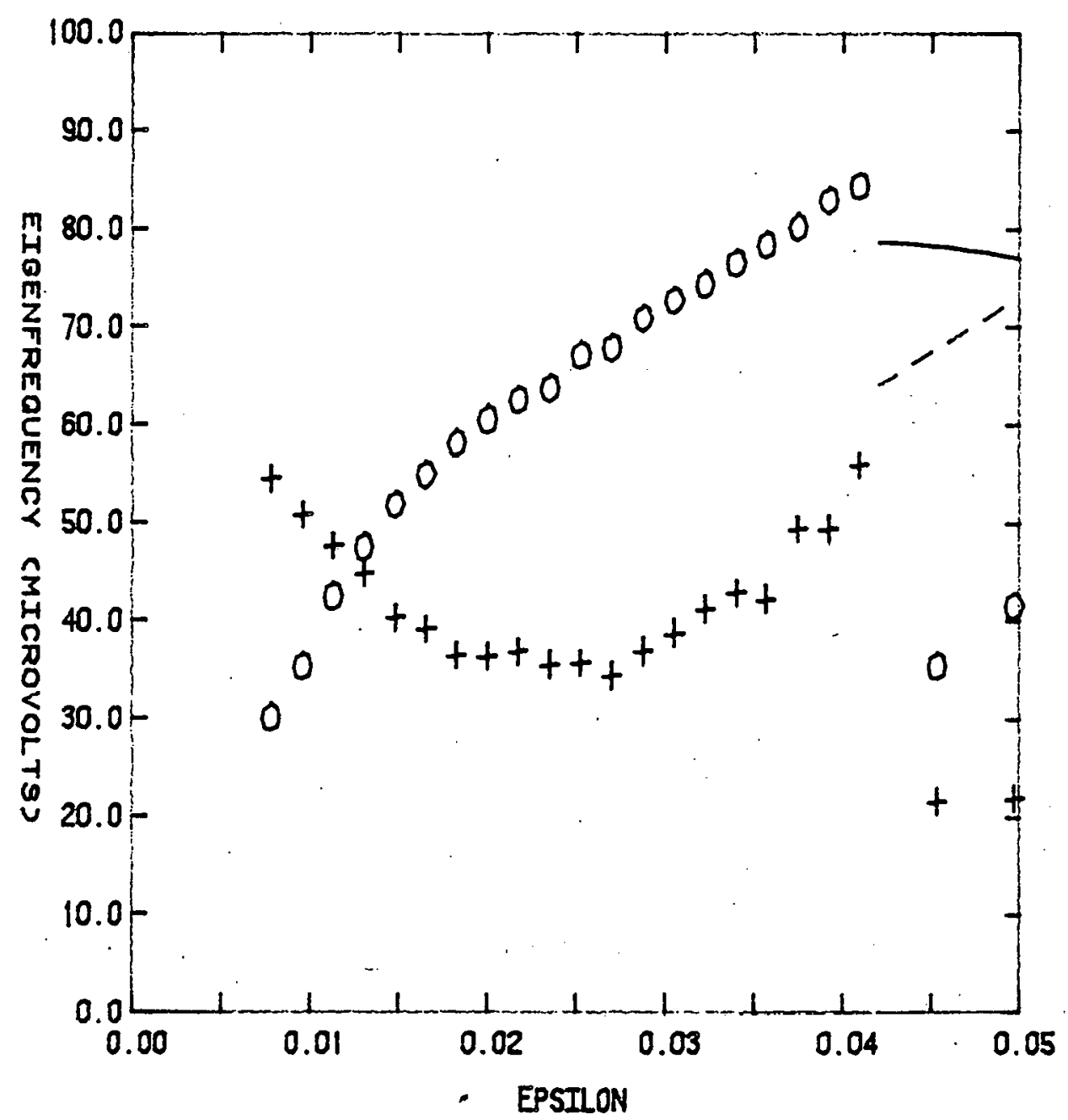

Figure 51. Real part ( 0 and solid) and imaginary part (t and dashed) of T-uivde dald and Dinter theory for $\$ 52$ with $\Gamma=26 \mu \mathrm{V}, \mathrm{Dk}^{2}=71.5 \mu \mathrm{V}$ and $\mathrm{T}_{\mathrm{c}}=1.147 \mathrm{~K}$. 


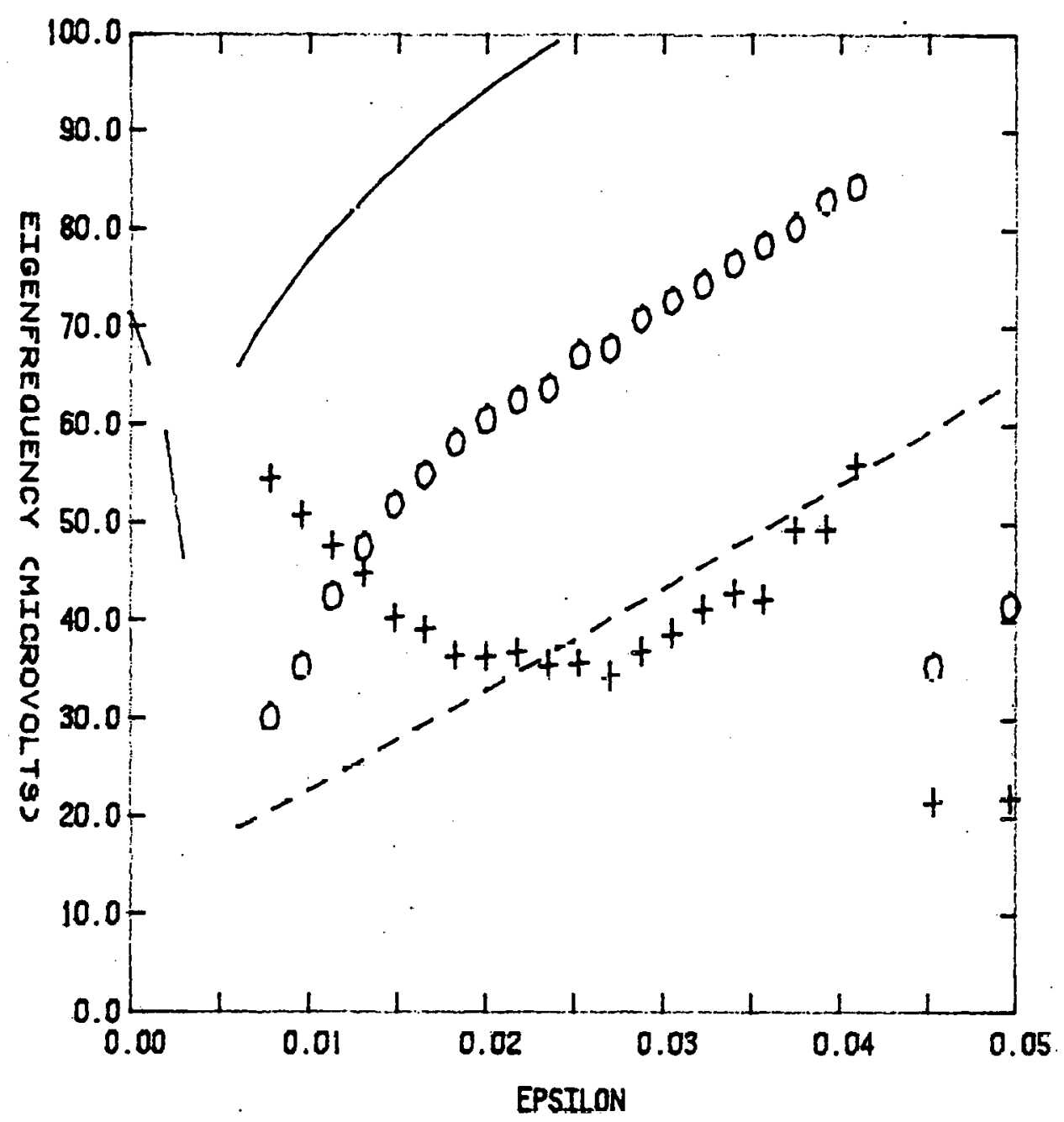

Figure 52. Real part ( 0 and solid) and imaginary part (t and dashed) of T-mode data and SA theory for $\$ 62$ with $\Gamma=26 \mu \mathrm{V}, \mathrm{Dk}^{2}=71.5 \mu \mathrm{V}$ and $\mathrm{T}_{\mathrm{c}}=1.147 \mathrm{~K}$. 
Orbach and Entin-Wohlman theory is in good agreement with the data with regard to the temperature at which the damping exceeds the resonant frequency and thus gives accurately the closing of the window of propagation. 


\section{The Current Carrying State}

Schön and Ambegaokar have calculated the eigenfrequencies in the presence of an impressed supercurrent. The solution of the coupled mode for $i \omega \gg 1 / \tau_{E}$ is given in Eqs. (138) and (142). The resonant frequency, $c k$ and the damping, $\omega_{D}$ are both reduced relative to the solutions for the uncoupled $(q=0)$ transverse mode. The primary reason for the decrease in these frequencies is the qdependence of the order parameter given in Eq. (109).

In Figs. (53) and (54) we plot the experimental eigenfrequencies for the transverse mode as a function of $I_{t r} / I_{c r}$ for two samples. Here $I_{t r}$ is the impressed transport current and $I_{c r}$ is the critical value of the transverse current. We believe $I_{c r}$ to be the current at which the eigenfrequency reaches a minimum.

The thenry is pintted as a function of $I_{\tau r} / I_{C r}$ by using the $q$-dependence of the supercurrent $I_{s} .60$

$$
I_{s} / I_{c r}=\left(3 q / 2 q_{c}\right)\left(1-q^{2} / 3 q_{c}^{2}\right)
$$

Although edges of the films are masked in making the tunneling junction, they may be able to support a larger critical current than the portion of the film probed by tunneling. If in spite of this we substitute the measured $I_{t r} / I_{c r}$ for $I_{s} / I_{c r}$ it should be valid to plot Eqs. (138) and (142) utilizing Eq. (188) as shown in Figs. (53) and (54).

The eigenfrequency which reduces to the high-frequency. longitudinal mode for $q=0$ is not easily extracted from Eq. (136). The low frequenty limit $\left(i \omega \ll 1 / \tau_{E}\right)$ of Eq. (136) which 


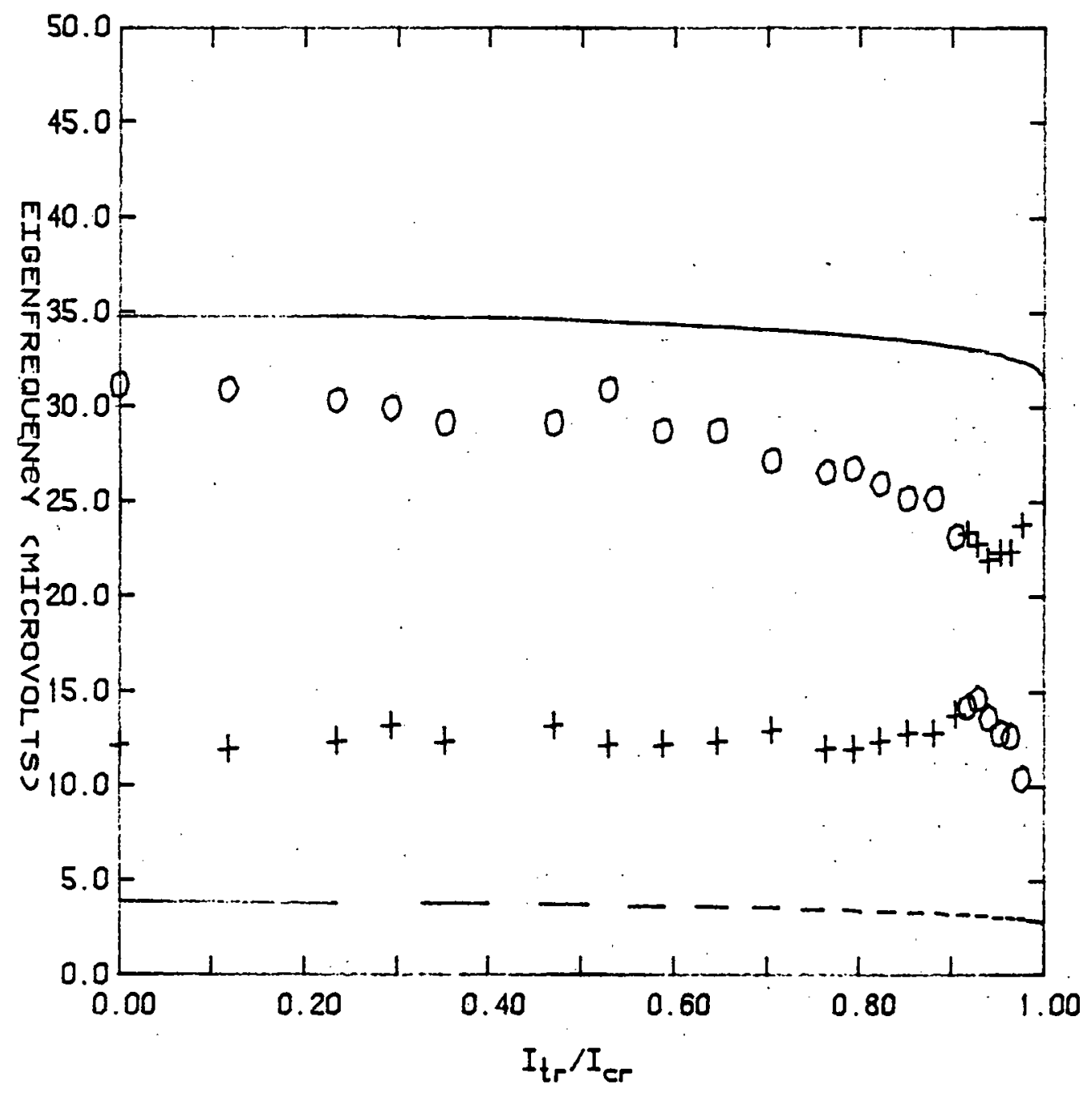

Figure 53. SA theóry for finite pair momentum and data for $\$ 54$ with $\Gamma=0, \mathrm{Dk}^{2}=27 \mu \mathrm{V}, \mathrm{H}=29.8 \mathrm{0e}, \mathrm{T}_{\mathrm{c}}=1.214 \mathrm{~K}$ and $\mathrm{T}=1.208 \mathrm{~K}$ for real part ( 0 and solid) and imaginary part ( + and dashed) of $\mathrm{T}$-mode. 


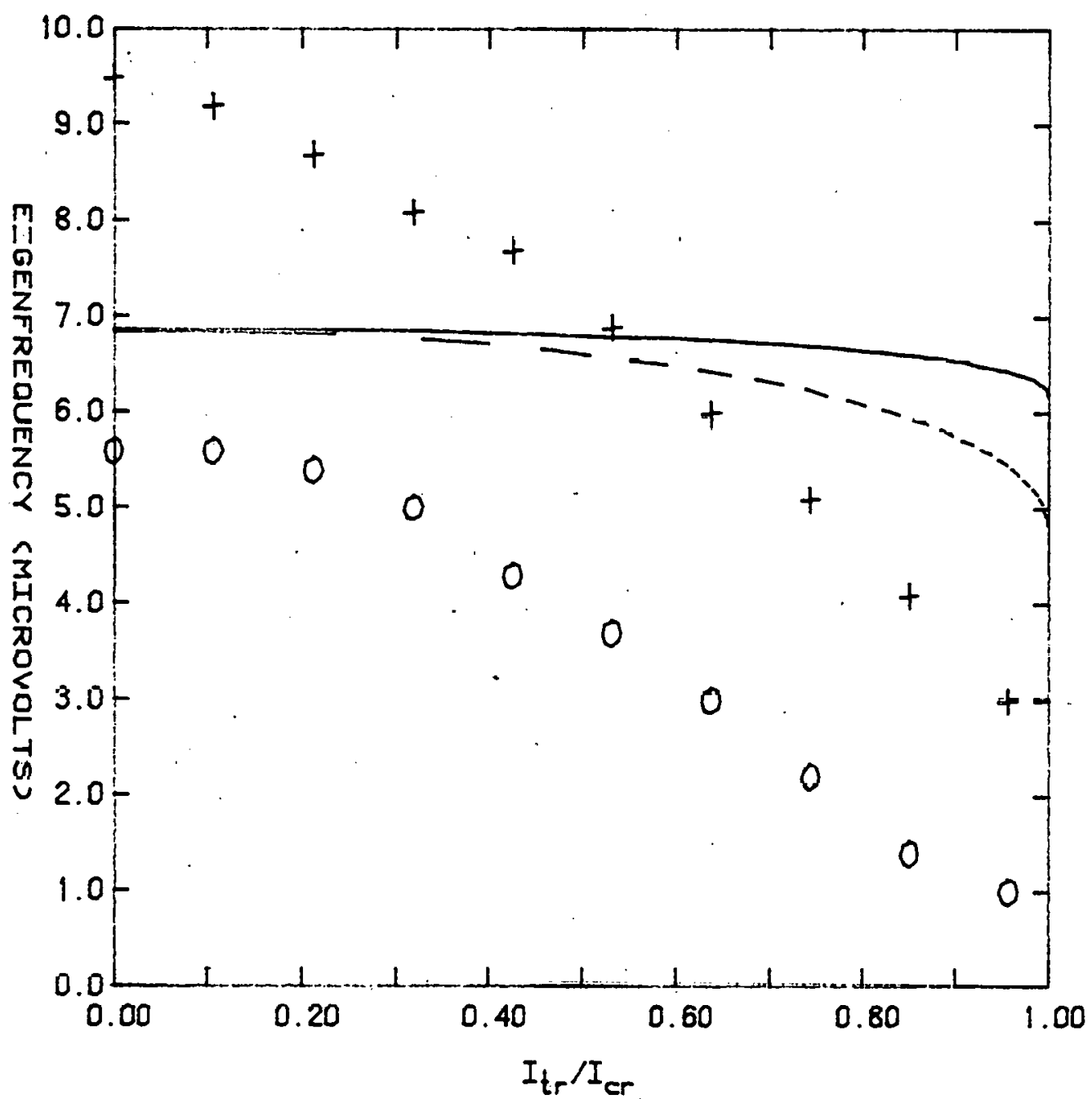

Figure 54. SA theory for finite pair momentum and data for $\$ 39$ with $\Gamma=26 \mu \mathrm{V}, \mathrm{Dk}^{2}=0.8 \mu \mathrm{V}, \mathrm{H}=9.9 \mathrm{Oe}, \mathrm{T}_{\mathrm{c}}=1.168$ and $T=1.160 \mathrm{~K}$ for real part ( 0 and solid) and imaginary part (+ and dashed) of T-mode. 
predicts mode softening for the longitudinal mode does not apply to Al. We therefore do not expect to be able to make any comparison of experiment and theory for the longitudinal mode in Al. 


\section{RELATED EXPERIMENTAL WORK}

Amato and McLean ${ }^{61}$ have extracted the temperature dependence of the order parameter relaxation time from third harmonic power generation of a paramagnetically doped superconductor in a strong microwave field. The measurements are taken for the $\mathrm{La}_{1-\mathrm{x}} \mathrm{Gd}_{\mathrm{x}} \mathrm{Sn}_{3}$ system and test the Gor'kov-Eliashberg theory ${ }^{62}$ for gapless superconductors which predicts a $\left(I-\left(T / T_{c}\right)^{2}\right)^{-1}$ divergence of the orderparameter rélaxation time. In the experiment third harmonic power generation results from the induced supercurrent $\vec{J}_{S} \propto|\Delta|^{2} \vec{A}$ where the superelectron density $|\Delta|^{2}$ is driven at twice the microwave frequency and the vector potential $\vec{A}$ is due to the electromagnetic radiation. For $\tau_{R} \omega \gg 1$, where $\tau_{R}$ is the order parameter relaxation time and $\omega$ is the microwave frequency, they show that $P_{3 \omega} \propto 1 /\left(\omega \tau_{R}\right)^{2}$ where $\mathrm{P}_{3 \omega}$ is the third harmonic power generated by the superconductor in the microwave cavity.

They claim the advantages of their measurements are: 1) freedom from geometry-dependent corrections, 2) only slight disturbance of the order-parameter from equilibrium, and 3) direct evidence of adiabatic response of the order-parameter to the electromagnetic field.

Although their measurements indicate that $T_{R}$ varies as $1 /\left(1-\left(\mathrm{T} / \mathrm{T}_{\mathrm{c}}\right)^{2}\right), \tau_{\mathrm{R}}$ is found to be an order of magnitude larger than predicted by the Gor'kov-Eliashberg theory. They attribute this to the fact that they are not in the limit $\tau_{s} T_{c} \ll 1$ (extreme gapless limit) but in fact have $\tau_{s} T_{c} \simeq 1$.

Song and $\mathrm{Kim}^{63}$ determined the order-parameter relaxation time 
by measuring the surface microwave resistance of a superconductor in the vortex state. The order-parameter relaxation rate is known 64,65 to be proportional to the slope $S=\left(H_{c 2} / R_{n}\right)\left(d_{f} / d H\right){ }_{H c 2}$ where $\mathrm{H}_{c 2}$ is the upper critical field, $\mathrm{R}_{\mathrm{n}}$ is the normal state resistance, $R_{f}$ is the flux-flow resistance and $H$ is the magnetic field. In the three systems $\mathrm{La}_{1-\mathrm{x}} \mathrm{Gd}_{\mathrm{x}} \mathrm{Al}_{2},\left(\mathrm{La}_{1-\mathrm{x}} \mathrm{Gd} \mathrm{x}_{3} \mathrm{Al}\right.$ and $\mathrm{La}_{1-\mathrm{x}^{\mathrm{Gd}}} \mathrm{x}^{\mathrm{Al}}{ }_{1.8^{\mathrm{In}}} 0.2$, they were able to show that for $\mathrm{x}=0$ the slope $S$ extrapolated to 0 at $T / T_{C}=1$ while for $x=0.002 \mathrm{~S}$ was larger and seemed to extrapolate to a finite value. The data is in qualitative agreement with calculations of Baba and Maki 64 which show that the order-parameter relaxation frequency should remain finite at $T=T_{c}$ for magnetically impure superconductors. Song and Kim claim that they observe finite relaxation times at $T_{c}$ because they are in a regime where the linear term of the Ginzburg-Landau theory dominates, while Amato and McLean measure a divergence because the nonlinear term becomes important in their experiments. The main physical difference between the two experiments is the degree of order-parameter perturbation. In the Amato-McLean experiment the order parameter only slightly deviates from its equilibrium value due to the microwave field. On the other hand in the Song-Kim experiment Cooper pairs are broken at the leading edge of a moving flux vortex and recombine at the trailing edge. Thus maximum departure from orderparameter equilibrium is achieved near each flux vortex. In an experiment ${ }^{66,67}$ that measures both the equilibrium value $\Delta$ and the relaxation time $\tau$ of the energy gap in $A l$, Schuller and 
Gray have determined that $\tau \propto \Delta^{-1}$ in agreement with the calculations of Schmid and Schön. ${ }^{15}$ The importance of measuring the equilibrium value of $\Delta$ is that close to $T_{c}, \Delta$ does not. have the $\left(T_{C}-T\right)^{1 / 2}$ temperature dependence predicted by the BCS theory.

In the experiment a $\mathrm{Sn}-\mathrm{Al}$ tunneling junction is current biased such that $\mathrm{V}=\Delta_{\mathrm{Sn}} \pm \Delta_{\mathrm{Al}}$. Since $\mathrm{dI} / \mathrm{dV}$ is very large in the vicinity. of the sum and difference gap features of the current-voltage characteristic a chänge in $\Delta$ will result in a corresponding change in $\mathrm{V}$ at fixed current bias. The voltage response is measured in real time and is found to exponentially relax to the equilibrium value once the aluminum film has been perturbed with a pulse of laser light.

The Schmid-Schön gap-relaxation time $\tau_{\Delta}$ (see Eq. (171)) is proportional to the inelastic electron scattering time $\tau_{F}$. The dara of Schuller and Gray are in quantitative agreement with the SchmidEchön relaration timo if $\tau_{E}$ ie taken to bo $7 \mathrm{nsec}$. which is in gond agreement with other measurements. ${ }^{41}$ 
D. CONCLUSIONS AND SUGGESTIONS FOR FUTURE WORK

From our measurements of the excess currents of a Josephson junction at temperatures near $T_{c}$ of the superconductor of interest we are able to extract the characteristic frequencies or order parameter fluctuations. The extreme accuracy required of our measurements to confirm the $\rho$-dependence of Eq. (182) allowed us to confirm the detailed temperature dependence of the orderparameter relaxation frequency for temperatures as high as $1: 2 \mathrm{~T}_{\mathrm{c}}$. The values of $\rho$ determined from the relaxation frequencies at $\mathrm{T}>\mathrm{T}_{\mathrm{c}}$ correlated very well with an independent determination based on measurements of the magnetic impurity concentration.

We are able to extract three characteristic frequencies from the excess current measurements at temperatures below $\mathrm{T}_{c} \cdot$ One of the frequencies is in excellent agreement with the $\rho, T, \Delta$ and $\mathrm{Dk}^{2}$ dependence of Eq. (101) which is a low-frequency longitudinal mode that we derive from Dinter's theory. We also derive a similar result from the Orbach and Entin-Wohlman theory in Eqs. (59) and (65), but agreement is not as good--particularly for large $\mathrm{Dk}^{2}$.

The complex frequency associated with the gap peak observed in the excess current is in excellent agreement with the longitudinal mode derived from the Schön and Ambegaokar theory and given in Eq. (124). 'l'he real part of $\omega$ is near $\Delta$ while the damping is given by $\Gamma$ in the gap regime. A similar expression could probably be derived from Dinter's theory since his expression for $s(\omega, k)$ contains a peak at $\omega \simeq \Delta$. Orbach and Entin-Wohlman derive an imagilialy frequency near $\Delta$, but since the data indicates there is 
a real frequency at $\Delta$ this solution is not entirely satisfactory. It is an alternate solution of their dispersion relation which is in reasonable agreement with the low-frequency mode.

The frequency which has been of most interest to the theorist is the transverse mode. The complexity of the associated equations necessitates making approximations which are very difficult to realize in practice. All of the theorists predict a propagating mode in the gap regime. The velocity $c$ given approximately by $(2 \Delta D)^{1 / 2}$ and the damping rate is $\pi \Delta^{2} / 4 \mathrm{~T}$ with $\rho, \Delta$ and $D k^{2}$-dependent corrections. We obtain qualitative agreement with each of the theories analyzed. Quantitative agreement is obtained for the damping given by Dinter's theory over its range of validity (see Table III).

This work can be extended in basically four directions: Experiments at low temperatures $(T \ll \Delta)$ should be carried out to test the theory of Holstein, Kulik and Orbach. ${ }^{48}$ This can best be achieved utilizing a dilution refrigerator, but may be approached by using ${ }^{3}$ He pot and/or studying a superconductor with higher $\mathrm{T}_{c}$. Secondly, the gapless superconductor $(\rho \gg 1)$ should be studied. The $\mathrm{Al}_{1-\mathrm{x}} \mathrm{Er} \mathrm{x}$ can be used for this if $\mathrm{x} \simeq 0.02$ and the sample is cooled to less than $0.4 \mathrm{~K}$. It would be of interest to test the theory in the clean limit, and finally, the limit $1 / \tau_{E} \gg \omega$ can be investigated using $\mathrm{Pb}, \mathrm{Sn}$ or In as the low- $\mathrm{T}_{c}$ electrode of an asymetric Josephson junction. Ideally a high- $T_{c}$ single-phase A-15 compound would be used as the high-T electrode for this experiment.

A related experiment is the direct observation of the propagating 
transverse mode by generating and detecting the mode with separate

junctions. It may be possible to observe the gap mode in the same way. 
The author expresses his deeply felt appreciation and gratitude to his thesis advisor, Professor Allen M. Goldman. His constant encouragement and patience were of great benefit for the completion of this work.

The author gratefully acknowledges the theoretical support and helpful discussions and correspondences with the following: Professor Ora Entin-Wohlman, Professor Ray Orbach, Professor Manfred Dinter, Dr. Gerd Schön, Professor V. Ambegaokar, Professor A. Schmid, Professor B. Patton, Professor J. Woods Halley and Professor Dan Dahlberg. The author acknowledges the support for this research by the Department of Energy on Contract. EY-76-S-02-1569.A002. The author also thanks the Office of Naval Research for providing the He gas used in this experiment.

The author also thanks his fellow graduate students who assisted in the experiment.

A special thanks is given to the author's wife, Marilyn, whuse love, encouragement and assistance were instrumental in accomplishing this work. 
APPENDIX A

$$
\text { In }{ }_{x} y \text { Barriers for Tunneling Junctions }
$$

One of the greatest difficulties of fabricating a high quality Josephson junction is to provide a thin yet uniform insulating barrier. It must be thin enough to allow substantial quantum mechanical tunneling of electron pairs and yet must be continuous and thick enough to prevent normal conduction of electrons. Much progress has been made by utilizing a semiconductor barrier ${ }^{68}$ in place of an insulating barrier. By using a material with a low barrier to conduction one can use a thicker barrier which is more . likely to be pin-hold free. Such a construction does allow substantial quantum mechanical tunneling while providing a continuous not-so-thin barrier but generally a great deal of undesirable ohmic conduction is also observed.

We have successfully fabricated natural oxide barriers on $\mathrm{Pb}_{1-\mathrm{x}} \mathrm{In}_{\mathrm{x}}$ alloys and artificial barriers of $\operatorname{In}_{\mathrm{x}} \mathrm{O}$ on $\mathrm{Al}$ and $\mathrm{Pb}$ by oxidizing thin In layers and by evaporating $\mathrm{In}_{2} \mathrm{O}_{3}$ directly. Representative fabrication parameters and junction characteristics have been reported elsewhere. 69

Eldridge and Matisoo ${ }^{70}$ disclosed a method of fahricating $\mathrm{Pb}_{1-\mathrm{x}} \mathrm{In}_{\mathrm{x}}-\mathrm{Pb}$ Josephson junctions with a mixed natural oxide of $\mathrm{Pb}$ and $\mathrm{In}$. The conductance was found to depend in a regular way 
on the oxide thickness and relative In to $\mathrm{Pb}$ content of the oxide. The advantages of alloying the $\mathrm{Pb}$ with In included suppression of detrimental hillock formation, and greater ease of oxidation resulting in more reliable barrier formation.

Our efforts to reproduce the work of Eldridge and Matisoo led to the idea of trying artificial In $\mathrm{O}_{\mathrm{y}}$ barriers. The $\mathrm{Pb}_{1-\mathrm{x}} \mathrm{In}_{\mathrm{x}}$ alloy was formed by deposition a thin film of In followed by a thin film of $\mathrm{Pb}$ to an atomic ratio of about 1 In : $10 \mathrm{~Pb}$. This bilayer film was heated to $130^{\circ} \mathrm{C}$ in vacuum for two hours. Auger analysis of one such film confirmed that In had interdiffused with the $\mathrm{Pb}$ and formed an In-r1ch oxide layer at the surface of the alloy in agreement with the observations of Eldridge and Matisoo. ${ }^{70}$ After this annealing step, the alloy was cooled to $30^{\circ}-40^{\circ} \mathrm{C}$ and 500 Torr of dry oxygen was admitted into the evaporation chamber for one to five hours. The oxygen was then removed and a $\mathrm{Bi}_{2} \mathrm{O}_{3}$ mask was evaporated leaving a $(0.27 \mathrm{~mm})^{2}$ area. The junction was completed by evaporating $1000 \AA$ of $A l$.

Junctions fabricated in this manner had normal resistances which increased from 1-100 ohms at room temperature to 5-50K ohms at 10 Kelvin. This large increase in resistance with decreasing temperature was attributed to the oxide being semiconducting rather than insulating. This conclusion was reinforced by the relatively large leakage conductance which was several percent of the normal conductance.

We attempted to make junctions with $\mathrm{R}_{\mathrm{N}}<1$ ohm using this. method, but these attempts always resulted in shorts. 
Results very similar to those described above were achieved if a 30 to $140 \AA$ film of In was deposited onto $1500 \AA$ of $\mathrm{Pb}$ and oxidized without prior thermal interdiffusion of the In and $\mathrm{Pb}$. These junctions were completed with $\mathrm{Bi}_{2} \mathrm{O}_{3}$ masking and $\mathrm{Pb}$ counterelectrodes as described above.

When Al was used as the base layer and was covered with a thin film of In it was discovered that only several minutes of oxidation at $30^{\circ} \mathrm{C}$ in oxygen at a pressure of 0.1 Torr was required. In contrast, several hours at $55^{\circ} \mathrm{C}$ in oxygen at a pressure of 250 Torr were required when $\mathrm{Pb}$ was the base layer. Junctions fabricated in this way exhibited leakage conductances of as 1 ittle as $0.1 \%$ of the normal conductance.

It was suspected that partial oxidation of the base electrode might be responsible for this observed dependence of the oxidation condition on the base electrode material.

We were also successful in fabricating Josephson junctions by direct sublimation of $\operatorname{In}_{2} \mathrm{O}_{3}$ onto $\mathrm{Al}$ and $\mathrm{Pb}$. The normal resistance did not depend on the base electrode material for these junctions but did depend on the pressure in the evaporation chamber during $\mathrm{In}_{2} \mathrm{O}_{3}$ sublimation. The normal resistance of the junction ranged from less than 1 ohm when the pressure was less than $10^{-7}$ Torr during sublimation to about 100 ohms when the pressure was about $3 \times 10^{-6}$ Torr.

We could not rule out the possibility that the base electrode was belng oxld1zed even for sublimated $\operatorname{In}_{2} \mathrm{O}_{3}$ barriers because we were never successful in fabricating $\mathrm{Ag}-\mathrm{Pb}$ or $\mathrm{Au}-\mathrm{Pb}$. junctions with 
In $O_{y}$ barriers. Attempts to do so resulted in shorts or conductance characteristics which had no feature at the $\mathrm{Pb}$ gap voltage.

Although this artificial barrier method does not appear to work for inert electrodes such as $\mathrm{Ag}$ and. Au we have shown that it can be a useful method for $\mathrm{Al}$ and $\mathrm{Pb}$. Furthermore, it is likely that it will work for other reactive metals such as $\mathrm{Sn}$, In and $\mathrm{Hg}$. 
APPENDIX B

$\mathrm{Pb} / \mathrm{Ag}$ Proximity Effect Tunneling Junctions

A method of introducing pair-breaking in a superconductor which is fundamentally different than spin-flip scattering is to force spatial variation of the order parameter. $26,27,72$ This can be accomplished in a number of ways. The one discussed in this appendix is fabrication of a proximity sandwich superconductor by pacing a normal metal in intimate contact with a superconductor. ${ }^{73,74}$ other examples, such as superconductors in a perpendicular magnetic field and the superconducting surface sheath, will not be considered here. For a review of this subject and further references see Ref. (27). One can intuitively understand the physical effect of placing a normal metal in intimate contact with a superconductor. If the thickness of the normal metal is less than a superconducting coherence length, it is not hard to imagine superconducting electron pairs penetrating the "normal" metal before breaking into normal electrons. When this happens, the "normal" metal is actually a superconductor since it contains a finite density of superconducting electrons. It is equally evident that the normal metal acts as a pair-breaker since it has no "pairing force" of its own.

This appendix contains a review of the theory which is relevant to thin proximity effect superconductors. A more comprehensive 
review of the theory is contained in Refs. (26), (27) and (72) and references therein. Following the theoretical review is a description of the method used to prepare $\mathrm{Pb} / \mathrm{Ag}-\mathrm{Pb}$ Josephson junctions and a discussion of the experimental results obtained from these junctions.

Theory

Proximity effect superconductors which are so thin that the order parameter does not depend on the coordinates normal to the interface are said to be in the Cooper limit. ${ }^{26}$ Theoretical calculations are much simpler in this limit and lend themselves to experimental tests. Our experiments were performed on proximity films consisting of $\sim 240 \AA$ of $\mathrm{Pb}$ deposited on $1250 \AA$ of Ag. Films this thin are well within the Cooper limit. The leakage range of Cooper pairs from the $\mathrm{Pb}$ into the $\mathrm{Ag}$ is

$$
\mathrm{K}^{-1}=\mathrm{v}_{\mathrm{N}} / 2 \pi \mathrm{T} \quad\left(\ell_{\mathrm{N}} \gg \zeta_{\mathrm{N}}\right)
$$

or

$$
\xi_{\mathrm{N}}=\left(D_{\mathrm{N}} / 2 \pi T\right)^{1 / 2} \quad\left(\ell_{\mathrm{N}}<<\xi_{\mathrm{N}}\right)
$$

where $D_{N}=v_{N} \ell_{N} / 3$ is the diffisuon constant and $v_{N}$ is the Fermi velocity in the normal metal. For $\mathrm{Ag}$ at $\mathrm{T}=1.2 \mathrm{~K}, \mathrm{~K}^{-1}=14000 \AA$ and $\xi_{\mathrm{N}}=2500 \AA$ if une Lakes $\ell_{\mathrm{N}}$ Lu be Llie Lhickitess of the normal $\operatorname{metal} \mathrm{d}_{\mathrm{N}}=1250 \AA$.

Fulde and Maki ${ }^{71}$ have calculated the tunneling probing thicknesses $\delta_{\mathrm{S}}$ and $\delta_{\mathrm{N}}$ for a thick, dirty proximity sandwich near its transition temperature $T_{C} \cdot \delta_{S}$ and $\delta_{N}$ are defined as the thickness 
of the layer in which a change in $\Delta(r)$ will have an effect on the tunneling characteristics. The subscripts $\mathrm{S}$ and $\mathrm{N}$ refer to the superconducting metal and normal metal sides respectively. They find

$$
\delta_{S}=\left(D_{S} / 2 \pi T\right)^{1 / 2}
$$

and

$$
\delta_{N}=\left(-K+\left(2 \pi T / D_{N}\right)^{1 / 2}\right)^{-1}
$$

For the $\mathrm{Ag} / \mathrm{Pb}$ film discussed above one finds $\delta_{S}=1000 \AA$ and $\delta_{N}=3000 \AA$. This again confirms that we are in the thin-film Cooper limit (since $\delta_{S} \gg d_{S}$ and $\delta_{N} \gg d_{N}$ ) and that a tunneling experiment will sample throughout the proximity sandwich.

The $\mathrm{Pb} / \mathrm{Ag}$ proximity system differs fundamentally from the $\mathrm{Al}_{1-\mathrm{x}} \mathrm{Er}_{\mathrm{x}}$ alloy system in two ways. (1) $\mathrm{Pb}$ is a strong coupling superconductor. Theoretical calculations ${ }^{75-78}$ indicate that strong coupling effects should enhance the Ginzburg-Landau diffusion rate by $20-40 \%$. One might expect that in a Cooper limit proximity sandwich the enhancement would be diluted by the negligible electronphonon interaction in the normal metal. (2) The pair-breaking mechanism is spatial variation of the order parameter rather than spin-flip scattering.

o. Entin-Wohlman ${ }^{79}$ has calculated the pair susceptibility for a proximity sandwich superconductor consisting of a weak-coupling superconductor in contact with a normal metal. Effects due to spinflip scattering are included in the theory but will: be omitted here for the sake of clarity. 
The relaxation frequency $\Gamma$ is found to be reduced from $\Gamma_{0}$, the relaxation frequency in solid, pure superconductors. The reduced frequency is

$$
\Gamma=\Gamma_{0} \frac{1+\operatorname{Rf}\left(\rho_{c}^{+}\right) \psi^{\prime}\left(1 / 2+\rho_{c}^{+}\right) / \psi^{\prime}(1 / 2)}{1+R \psi^{\prime}\left(1 / 2+\rho_{c}^{+}\right) / \psi^{\prime}(1 / 2)}
$$

where $\Gamma_{0}$ and $f(p)$ are given by Eqs. (8) and (17) respectively and $\psi^{\prime}$ is the trigamma function. $\mathrm{R}$ is given by

$$
R=d_{N} N_{N} / d_{S} N_{S}
$$

where $d_{N}$ and $d_{S}$ are the thicknesses of the normal and superconducting layers and $\mathrm{N}_{\mathrm{N}}$ and $\mathrm{N}_{\mathrm{S}}$ are the respective densities of states at the Fermi level. The parameter $\rho_{c}^{+}$is given by

$$
\rho_{c}^{+}=(1+R) / 2 \pi K_{B} T_{c} \tau_{N}
$$

where $T_{C}$ is the transition temperature of the sandwich and $\tau_{N}$ is given by

$$
\tau_{N}=L_{N} / v_{N} \sigma
$$

Here $\sigma$ is the transmission probability for an electron at the $S-N$ interface, $v_{N}$ is the Fermi velocity in $N$ and $\bar{L}_{N}$ is the mean path between collisions with the interfacial barrier given by

$$
\begin{array}{ll}
\mathrm{L}_{\mathrm{N}} \simeq 4 \mathrm{~d}_{\mathrm{N}} \quad\left(l \gg \mathrm{d}_{\mathrm{N}}\right) \\
\mathrm{L}_{\mathrm{N}} \simeq 4 \ell \quad\left(l<\mathrm{d}_{\mathrm{N}}\right)
\end{array}
$$


where $\ell$ is the electron mean-free-path.

In order to calculate the relaxation frequency from Eq. (B5) one need only know $R$ and $\rho_{c}^{+}$. $R$ is easily determined via Eq. (B6). In principle one can determine $\rho_{C}^{+}$from Eqs. (B7) - (B9) if the transmission probability $\sigma$ is known. In general, however, $\sigma$ depends on the transition temperature, $R$, the BCS coupling constants $\lambda_{S}$ and $\lambda_{\mathrm{N}}$ for $\mathrm{S}$ and $\mathrm{N}$ respectively and on strong-coupling corrections. ${ }^{80-83}$ The complicated dependence on all of these parameters is avoided in the weak-coupling limit for $\lambda_{N}=0$. In this limit McMillan ${ }^{80}$.finds

$$
\ln \left(T_{c o} / T_{c}\right)=\left[\psi\left(-1 / 2+\rho_{c}^{+}\right)-\psi(1 / 2)\right] /(1+R)
$$

Eqs. (B5) and (BI1) offer a simple means of calculating the order parameter relaxation frequency in the weak-coupling limit. It is not unreasonable to assume a similar set of equations will describe the more general case with $\rho_{c}^{+}$being renormalized in terms of the relevant physical parameters.

\section{Experimental}

The expcriment will be cunsidered in three parts. First we will discuss the construction of a suitable bilayer film, then the incorporation of such films as electrodes in tunneling junctions, and finally the tunneling data obtained from such junctions.

The requirements for the proximity effect superconductor are rather stringent. A bilayered fịm can result in a good proximity effect superconductor only if there is good metallic contact between the two metals (no intervening oxide or other contamination) and 
no interdiffusion of the two metals. Additional requirements for the particular experiments in which we are interested, with the constraints of our apparatus, are that $T_{c}$ be much less than $T_{c o}$ and greater than $1.10 \mathrm{~K}$. We would not expect films with $\mathrm{T}_{c} \leqslant \mathrm{~T}_{\text {co }}$ to have a measurably reduced $\Gamma$, and because of the limited temperature range of our apparatus we would not be able to take sufficient data on f1lms with $T_{c}<1.10 \mathrm{~K}$. Furthermore we require that each layer be sufficiently thin to ensure that the order parameter be nearly constant throughout. Large variations in $\Delta$ across the thickness of the f1lm would complicate the analysis.

The $\mathrm{Pb} / \mathrm{Ag}$ system was chosen since it could readily meet all of our requirements. As mentioned before we found that $240 \AA$ of $\mathrm{Pb}$ on $1250 \AA$ of $\mathrm{Ag}$ resulted in a proximity effect superconductor with $T_{c} \simeq 1.4 \mathrm{~K}$, which is low enough to significantly reduce the orderparameter relaxation frequency.

In FIg. (5j) we plot $T_{c}$ of bilaycr films as a function of $d_{S}$. For all of these films $d_{N}=1250 \pm 50 \AA$. It is important to note that in the range of interest $T_{c}$ varies by $25 \mathrm{mK}$ for each angstrom of $\mathrm{Pb}$. This may be a severe problem in this experiment, because we are looking at quantities which are sensitive to changes in $T$ of $1 \mathrm{mK}$. We, in fact, see a rather large transition region $(\sim 0.1 \mathrm{~K})$ in many of the samples.

To fabricate the junction we adopted the method of Garno 84 in which he oxidizes a $\mathrm{Pb}$ film in a controlled humidity chamber to provide a natural oxide barrier for a tunneling junction. We found his method very reliable and reproducible. We could 


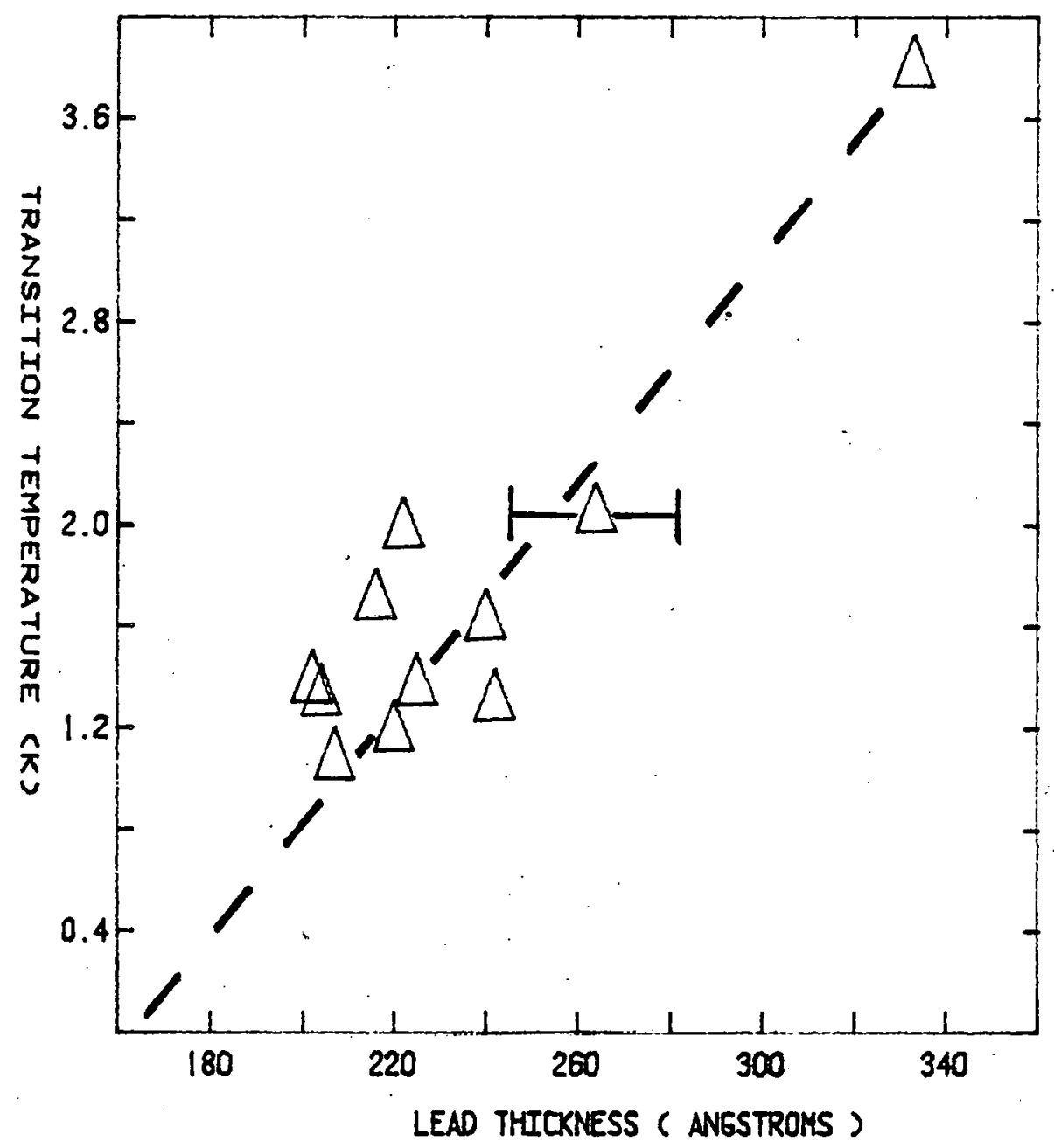

Figure 55. Plot of $\mathrm{T}_{c}$ vs $\mathrm{Pb}$ film thickness for a number of $\mathrm{Ag} / \mathrm{Pb}$ proximity superconductors. The thickness of the Ag film is $1250 \AA$. 
consistently make good $\mathrm{Pb}-\mathrm{Pb}$ junctions with $\mathrm{R}_{\mathrm{N}} \sim 0.5 \Omega$ by oxidizing for thirty minutes at $73 \%$ relative humidity.

It was, however, far more difficult to reliably oxidize the $\mathrm{Pb} / \mathrm{Ag}$ bilayer films. Very often the junction was shorted and at best suffered from a relatively large leakage conductance. We noted in Appendix A that our attempts to fabricate ( $\mathrm{Ag}$ or $\mathrm{Au}$ ) - ( $\operatorname{In}_{\mathrm{x}} \mathrm{O}_{\mathrm{y}}$ or $\left.\mathrm{Bi}_{2} \mathrm{O}_{3}\right)$ - $\mathrm{Pb}$ junctions resulted in shorts. For this reason it may have been difficult to reliably mask the edges of the $\mathrm{Pb} / \mathrm{Ag}$ film unless the $\mathrm{Pb}$ and $\mathrm{Ag}$ films are prefectly superimposed.

Nevertheless we were able to measure the excess current for several junctions. The excess current for a proximity effect junction well above its transition temperature fits the simple quasi-Lorentzian structure observed for $\mathrm{Al}$ and $\mathrm{Al}_{1-\mathrm{x}} \mathrm{Er} \mathrm{x}$. However close to $\mathrm{T}_{\dot{f}}$ it appears that in some cases the excess current may include two or more peaks. In Fig. (56) we plot a typical $I_{\mathrm{ex}}(\mathrm{V})$ curve with a quasi-Lorentzian superimposed.

In Table IV we summarize the parameters of several $\mathrm{Pb} / \mathrm{Ag}-\mathrm{Pb}$ junctions. In Fig. (5.7) we plot the location of the peak vs. T-T for the four samples which did nor have larye leakage conductances. In Fig. (58) we plot the data normalized to $3\left(\mathrm{~T}-\mathrm{T}_{\mathrm{C}}\right) / \pi$ which is the Ginzburg-Landau diffusion rate for an unperturbed weak-cuupled superconductor near $T_{c}$. The projected intercept of the data with $\mathrm{T}-\mathrm{T}_{\mathrm{C}}=0$ represents the factor by which the order parameter is reduced as given in Eq. (B5).

One can calculate $\rho_{c}^{+}$from Eq. (B11) along with the known values of $T_{c}$ and $R$. Using this value of $\rho_{c}^{+}$one can then calculate the 


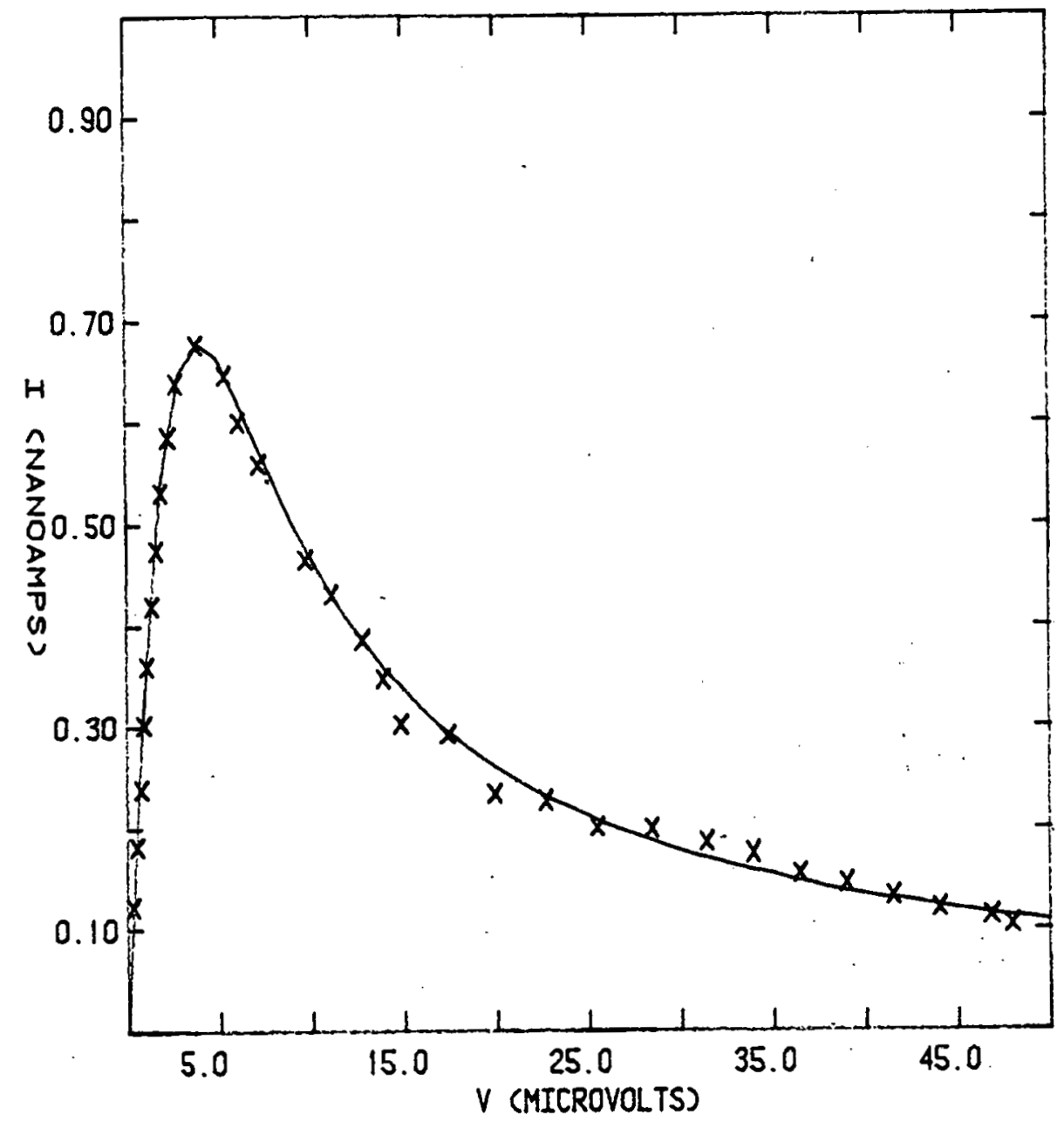

Figure 56. Representative plot of $I_{\text {ex }}$ vs $V$ for an $\mathrm{Ag} / \mathrm{Pb}-\mathrm{Pb}$ junction with quasi-Lorentzian superimposed. 
TABLE IV

Physical Parameters of Several $\mathrm{Ag} / \mathrm{Pb}$ - $\mathrm{Pb}$ Tunneling Junctions

\begin{tabular}{|c|c|c|c|c|c|c|c|}
\hline $\begin{array}{l}\text { Sample } \\
\text { Number }\end{array}$ & $\begin{array}{l}{ }_{\mathrm{Ag}}^{\mathrm{Ag}} \\
(\AA)\end{array}$ & $\begin{array}{l}\mathrm{d}_{\mathrm{Pb}} \\
(\AA)\end{array}$ & $\begin{array}{l}T_{c} \\
(\mathrm{~K})\end{array}$ & $\underset{\text { (ohms) }}{\mathrm{R}_{\mathrm{N}}}$ & $\mathrm{R}_{\mathrm{N}} \mathrm{G}_{\mathrm{L}} *$ & $\begin{array}{l}\text { Edge } \\
\text { mask }\end{array}$ & $\begin{array}{l}\text { Area } \\
\left(\mathrm{mm}^{2}\right)\end{array}$ \\
\hline 98 & 1250 & 333 & 3.80 & 0.2 & 0.7 & $\mathrm{Bi}_{2} \mathrm{O}_{3}$ & $0.27 \times 0.27$ \\
\hline 99 & 1250 & 225 & 1.36 & 0.032 & 0.044 & $\mathrm{Bi}_{2} \mathrm{O}_{3}$ & $0.27 \times 0.27$ \\
\hline 103 & 1250 & 220 & 1.18 & 0.14 & 0.88 & $\mathrm{CaF}_{2}$ & $0.27 \times 0.27$ \\
\hline 123 & 1140 & 225 & 2.80 & 0.11 & 0.55 & $\mathrm{Bi}_{2} \mathrm{D}_{3}$ & $0.27 \times 0.27$ \\
\hline 131 & 1250 & 222 & 1.98 & 0.32 & 0.70 & collodion & $1.58 \times 0.13$ \\
\hline $138 a$ & 1250 & 207 & 1.07 & 64. & 0.91 & Sio & $0.27 \times 0.27$ \\
\hline $141 b$ & 1250 & 216 & 1.70 & 0.63 & 0.042 & $\mathrm{Bi}_{2} \mathrm{O}_{3}$ & $0.27 \times 0.13$ \\
\hline $142 a$ & 1250 & 202 & 1.37 & 3. & 0.37 & Sio & $0.27 \times 0.13$ \\
\hline $143 a$ & 1250 & 204 & 1.32 & 0.7 & 0.41 & $\mathrm{Bi}_{2} \mathrm{O}_{3}$ & $0.27 \times 0.13$ \\
\hline $143 \mathrm{~b}$ & 1250 & $2 / 10$ & 1.31 & 0,74 & 0.053 & $\mathrm{Bi}_{2} \mathrm{O}_{3}$ & $0.27 \times 0.13$ \\
\hline $143 c$ & 1250 & 264 & 2.05 & 0.9 & 0.008 & $\mathrm{Bi}_{2} \mathrm{O}_{3}$ & $0.27 \times 0.13$ \\
\hline 144 & 450 & 111 & $<1.6$ & 0 & - & $\mathrm{Bi}_{2} \mathrm{O}_{3}$ & $0.27 \times 0.13$ \\
\hline 145 & 450 & 146 & 2.1 & 0 & - & $\mathrm{Bi}_{2} \mathrm{O}_{3}$ & $0.27 \times 0.13$ \\
\hline 146 & 450 & 200 & 3.0 & 0.28 & 0.7 & $\mathrm{Bi}_{2} \mathrm{O}_{3}$ & $0.27 \times 0.13$ \\
\hline 155 & 560 & 175 & 1.94 & 0.48 & .024 & $\mathrm{Bi}_{2} \mathrm{O}_{3}$ & $0.27 \times 0.13$ \\
\hline 173 & 1250 & 242 & 1.27 & 2.9 & 0.0003 & $\mathrm{Bi}_{2} \mathrm{O}_{3}$ & $0.27 \times 0.13$ \\
\hline
\end{tabular}

$*_{G}$ is the leakage conductance of the junction. 


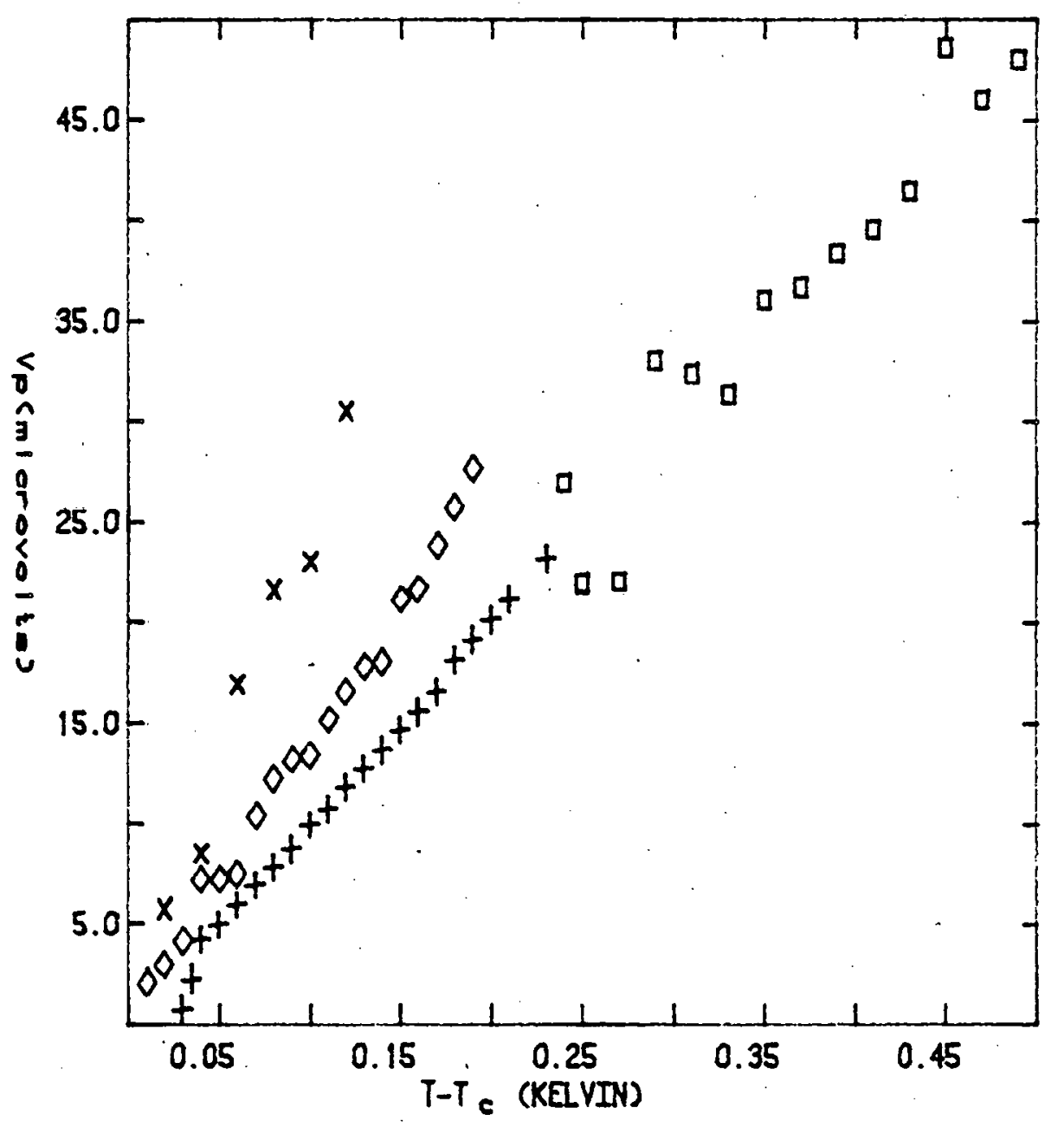

Figure 57. Plot of $\mathrm{V}_{\mathrm{p}}$ vs $\mathrm{T}-\mathrm{T}_{\mathrm{c}}$ for four $\mathrm{Ag} / \mathrm{Pb}-\mathrm{Pb}$ junctions. Samples $\$ 1.43 \mathrm{~b}(\mathrm{O}), 143 \mathrm{C}(0), 155(\mathrm{X})$, and $173(+)$. 


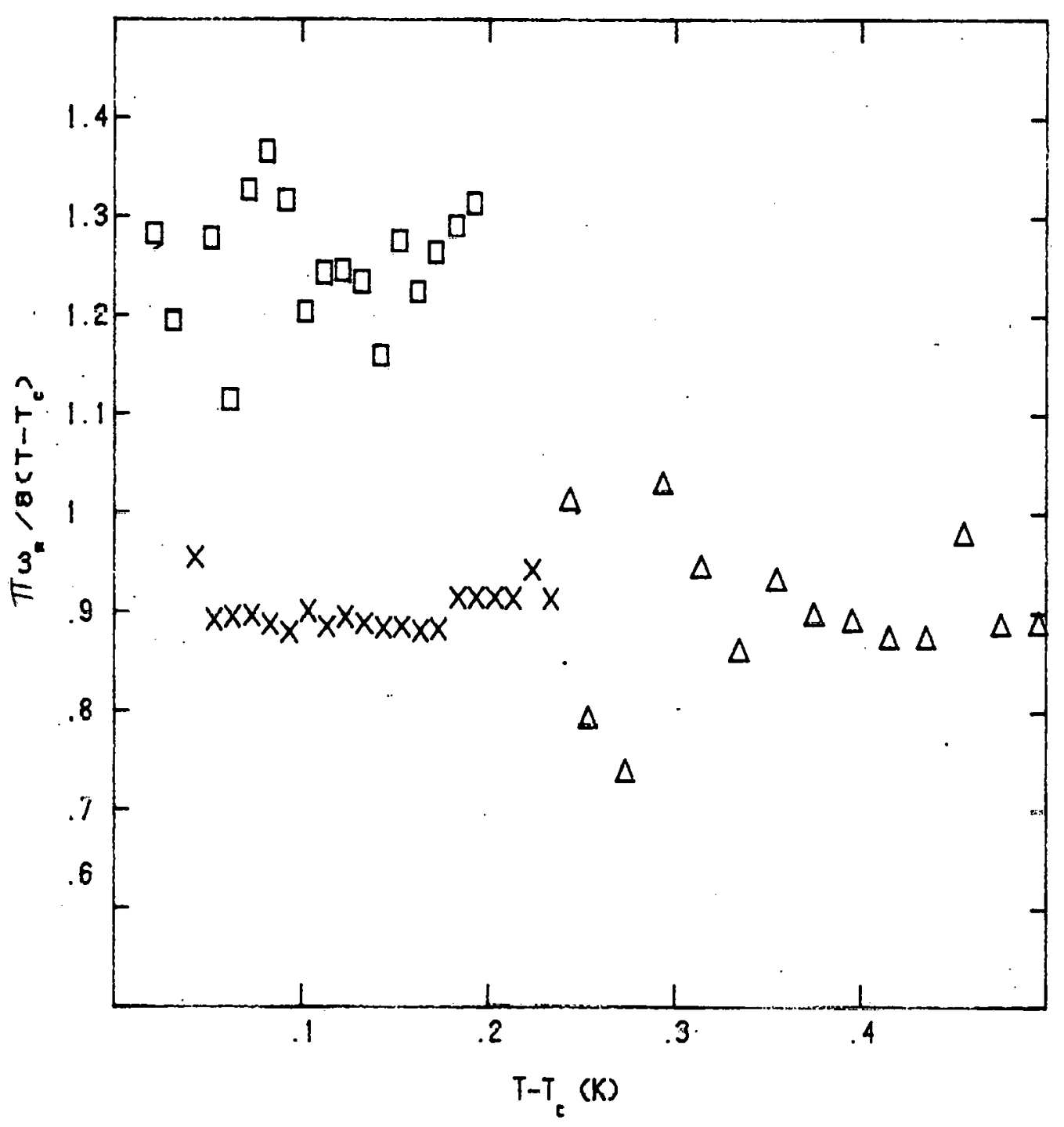

Figure 58. Plot of $\omega_{R}$ normalized to $8\left(T-T_{c}\right) / \pi$ plotted as a function of $\mathrm{T}-\mathrm{T}_{\mathrm{c}}$ for three $\mathrm{Ag} / \mathrm{Pb}-\mathrm{Pb}$ junctions. Samples $\#_{143 \mathrm{~b}}(\Delta), 143 \mathrm{C}(\square)$, and $173(\mathrm{X})$. 
ratio $\Gamma / \Gamma_{0}$ from Eq. (B5). The results of such a calculation along with the experimental values for four films determined from Fig! (58) are summarized in Table V. The data for samples number $143 \mathrm{~b}$ and 173 are in excellent agreement with theory. The quality of the data for sample 非173 was especially good having error bars small enough to show credible agreement with theory. The data for samples $143 \mathrm{c}$ and 155 seem to be at odds with the theory. The data for sample \#155 consists of only six points which were taken over a limited temperature range. We have no explanation for the apparent discrepancy in saraple $\# 143 c$.

Our efforts to measure the relaxation frequency below the transition temperature were plagued by the inability to completely suppress the dc Josephson effect without driving the $\mathrm{Pb} / \mathrm{Ag}$ film normal. The critical field near $T_{C}$ for sample $143 \mathrm{C}$ was measured to be

$$
H_{c}(T)=136\left(T_{c}-T\right)^{1 / 2} \text { oe } k^{-1 / 2} \text {. }
$$

The coherence length can be determined from the magnetic field. dependence of the diffusion rate above $T_{c}$. For sample 173 we found

$$
\xi(T)=3760 \AA / \varepsilon^{1 / 2} \text {. }
$$

Since the BCS coherence length for $\mathrm{Pb}$ is only $830 \AA$, we believe the mean free path is long and the proximity films are in the clean 1imit. 


\section{TABLE V}

Summary of the Parameters Relevant to the

Ginzburg-Landau Diffusion Rate

\begin{tabular}{cccccc}
\hline $\begin{array}{c}\text { Sample } \\
\text { Number }\end{array}$ & $\begin{array}{c}\mathrm{T}_{\mathrm{c}} \\
(\mathrm{K})\end{array}$ & $\mathrm{R}$ & $\begin{array}{c}\rho_{\mathrm{c}}^{+} \\
(\mathrm{calc})\end{array}$ & $\begin{array}{c}\Gamma / \Gamma_{0} \\
(\mathrm{calc})\end{array}$ & $\begin{array}{c}\dot{\Gamma} / \Gamma_{0} \\
(\operatorname{expt})\end{array}$ \\
\hline $143 \mathrm{~b}$ & 1.307 & 1.441 & 0.306 & 0.865 & $0.9 \pm 0.2$ \\
$143 \mathrm{c}$ & 2.048 & 1.310 & 0.251 & 0.882 & $1.2 \pm 0.1$ \\
173 & 1.269 & 1.429 & 0.308 & 0.865 & $0.87 \pm 0.03$ \\
155 & 1.944 & 0.885 & 0.227 & 0.912 & $2.3 \pm 0.2$ \\
\hline
\end{tabular}

The parameters $R$ and $\rho^{+}$are calculated from Eqs. (B6) and (BII) respectively. These values are then used to calculate $\Gamma / \Gamma_{0}$ using Eq. (B5). 


\section{REEERENCES}

1. R. A. Ferre11, J. Low Temp. Phys. 1, 423 (1969).

2. D. J. Scalapino, Phys. Rev. Lett. 24, 1052 (1970).

3. R. V. Carlson, Doctoral Dissertation, University of Minnesota, unpublished.

4. R. V. Carlson and A. M. Goldman, J. Low Temp. Phys. 25, 67 (1976).

5. G. Giaquinta and N. A. Mancini, Rivista Del Nuovo Cimento $\underline{1}$, No. 9, 1 (1978).

6. I. Giaver, Tunneling Phenomena in Solids, E. Burstein and S. Lundquist editors (Plenum, New York, 1969), Chapter 19.

7. G. M. Eliashberg, Zh. Eksp. Teor. Fix. 34, 668 (1972) and references therein.

8. J. T. Anderson, Doctoral Dissertation, University of Minnesota (1971), unpublished.

9. J. T. Anderson and A. M. Goldman, Phys. Rev. Letters 25, 743 (1970).

10. K. Yoshihiro and K. Kajimura, Phys. Lett. 32A, 71 (1970).

11. S. G. Lipson, C. G. Kuper and A. Ron, Physica 55, 269 (1971).

12. J. T. Anderson, R. V. Carlson, dind A. M. Goldman, J. Low Temp. Phys. $\underline{8}, 29$ (1972).

13. R. V. Carlson and A. M. Goldman, Phys. Rev. Lett. 31,880 (1973).

14. R. V. Carlson and A. M. Goldman, Phys Rev Lett. 34, 11 (1975).

15. A. Schmid and G. Schön, J. Low Temp. Phys. 20, 207 (1975).

16. A. Schmid and G. Schön, Phys. Rev. Lett. 34, 941 (1975).

17. A. J. Bray and H. Schmidt, J. Low Temp. Phys. 21, 669 (1975). 
18. A. J. Bray and H. Schmidt, Solid State Com. 17, 1175 (1975).

19. K. Maki and H. Sato, J. Low Temp. Phys. 16, 557 (1974).

20. G. Brieskorn, M. Dinter and H. Schmidt, Solid State Com. $\underline{15}$, 757 (1974).

21. E. Simanek and J. C. Hayward, Physica 78, 199 (1974).

22. G. A. Baramidze and O. D. Cheishvili, Sov. JLTP 1, 430 (1975).

23. G. Burrafato, et al., Phys. Status Sol. 81B, 578 (1977).

24. M. Dinter, J. Low Temp. Phys. 32, 529 (1978).

25. G. Schön and V. Ambegeokar, Phys. Rev. B, 1y, 3513 (1979).

26. K. Maki, Superconductivity, R. D. Parks editor (Marcel Dekker, Inc., New York, 1969) Chapter 18.

27. P. Fulde, Tunneling Phenomena in Solids, E. Burstein and $S$. Lundqvist editors (Phenum, New York, 1969) Chapter 29.

28. M. B. Maple, Magnetism Vol. V, G. T. Rado and H. Suhl editors (Academic Press, New York and London, 1973) Chapter 10.

29. P. W. Anderson, J. Phys. Chem. Solids 11, 26 (1959).

30. A. A. Abrikosov and L. P. Gor'kov, Zh. Eksperim. i Teor. Fiz. 35, 1558 (1958); Soviet Phys. JETP 8, 1090 (1959).

31. For exceptlons to this and furthar discussion see pages 432435 of Ref. 32 or discussion of equivalence theorems beginning on pages 1073 and 1089 of Ref. 31 .

32. A. A. Abrikosov and I. P. Gor'kov, Zh. Eksperim. i Teor. Fiz 39, 1781 (1960); Phys. JETP 12, 1243 (1961).

33. O. Entin-Wohlman and R. Orbach, Phys. Rev. B 12, 4812 (1975).

34. H. Schmidt, Phys. Lett. A 27, 658 (1968).

35. This argument is due to D. Scalapino and conveyed to us by R. Orbach.

36. R. A. Craven, G. A. Thomas and R. D. Parks, Phys. Rev. B $\underline{4}$, 2185 (1971).

37. 0. Entin-Wohlman and R. Orbach, Ann. Phys. (New York 116, 35 (1978). 
38. M. Dinter, J. Low Temp. Phys. 26, 39 (1977).

39. Ibid., equation 18 .

40. V. Ambegaokar, Phys. Rev. Lett. 39, 235 (1977).

41. S. B. Kaplan, C. C. Chi, D. N. Langenberg, J. J. Chang, S. Jafiarey and D. J. Scalapino, Phys. Rev. B 14, 4854 (1976).

42. A. M. Goldman proposes to test this using a double junction arrangement.

43. S. N. Artemenko and A. F. Volkov, Zh. Eksp. Teor. 69, 1764 (1975); Sov. Phys.-JETP 42, 896 (1976).

44. V. P. Galaiko, N. I. Glushchik and V. S. Shumeiko, Sov. J. Low Temp. Phys. 4, 139 (1978).

45. O. D. Cheishv1li, Sov. J. Low Temp. Phys. 3, 357 (1977).

46. C. J. Pethick and H. Smith, Journal De Physique 39, 488 (1978).

47. A. M. Kadin, W. J. Skocpol and L. N. Smith, "Charge Imbalance Waves and Nonequilibrium Dynamics Near a Superconducting PhaseSlip Center," J. Low Temp. Phys., to be published.

48. T. Holstein, I. O. Kulik and R. Orbach, "Pair Susceptability and Mode Propagation in Superconductors: A Microscopic Approach," preprint."

49. A. Schmid, Phys. Kond. Mater 8, 129 (1968).

50. W. E. Lawrence and A. B. Meador, Phys. Rev. B 18, 1154 (1978).

51. All metalo with the exception of Al shot were obtained from Alpha Products, Ventron Corporation, Danvers, Massachusetts. The Al shot was obtained from ROC/RIC.

52. General Electric Handbook of Binary Alloys.

53. Crest Epoxy $\$ 7410$ and $\# 3170$, Crest Product Corporation, 2200 South Susan Street, Santa Ana, California 92704.

54. Wire from Supercon, Inc., 9 Erie Drive, Nantick, MA 01760.

55. Calibrated germanium resistors were purchased from Cryocal Incorporated, Eden Prairie, Minnesota.

56. Tunneling. Phenomena in Solids (Edited by E. Burstein and S. Lundquist) p. 260 . 
57. P. R. Bevington, Data Reduction and Error Analysis for the Physical Sciences (McGraw-Hill, New York, 1969), and references therein.

58. C. Rettori, et al., Phys. Rev. B I, I (1973).

59. F. Aspen and A. M. Goldman, Phys. Rev. Lett. 43, 307 (1979). Eq. (2) therein is in error by a factor of $\pi / 2$.

60. V. Ambegaokar, Superconductivity, edited by P. R. Wallace (Gordon and Breach, New York, 1969), p. 117.

61. J. C. Amato and W. L. Mclean, Phys. Rev. Lett. 37, 430 (1976).

62. L. P. Gor'kov and G. M. Eliashberg, Zh. Eksp. Teor. Fiz. 54, 612 (1968); Sov. Phys. JETP 27, 328 (1968).

63. Y. Song and Y. B. Kim, Solid State Com. 25, 69 (1978).

64. Y. Baba and K. Maki, Prog. Theoret. Phys. (Kyoto) 44, 1431 (1970).

65. G. Fischer, R. D. McConnell, P. Monceau and K. Maki, Phys. Rev. B I, 2134 (1970); and Phys. Rev. B 3, 1617 (1970).

66. I. Schuller and K. E. Gray, Solid State Com. 23, 337 (1977).

67. I. Schuller and K. E. Gray, Phys. Rev. Lett. 36, 429 (1976).

68. W. H. Keller and J. E. Nordem, J. Appl. Phys. 44, 4732 (1973).

69. F. Aspell dill A. M. Coldman, Cryogeniss, 72.1 (1.976).

70. J. M. Eldridge and J. Matisoo, U.S. Patent $3,816,173$.

71. P. Fulde and K. Maki, Phys. Rev. Letters 15, 675 (1965).

72. G. Deutscher and P. G. de Gennes, Superconductivity, R. D. Parks ed. (Marcel Deckker, Inc., New York, 1969) Chapter 17.

73. P. G. de Gennes and E. Guyon, Phys. Letters 3, 168 (1963).

74. N. R. Werthamer, Phys. Rev. 132, 2440 (1963).

75. N. Menyhárd, Il Nuovo Cimento 44B, 4561 (1966).

76. G. Eilenberger and V. Ambegaokar, Phys. Rev. 158, 332 (1967).

77. P. Fulde and K. Maki, Phys. Kandens. Materie $\underline{8}, 371$ (1969).

78. D. Rainer and G. Bergmann, J. Low Temp. Phys. 14, 501 (1974). 
79. 0. Entin-Wohlman, Phys. Rev. B 14, 274 (1976).

80. W. L. McMillan, Phys. Rev. 175, 537 (1968).

81. P. G. de Gennes, Rev. Mod. Phys. 36, 225 (1964).

82. W. Silvert, Phys. Rev. B 12, 4870 (1975).

83. G. Deutcher, O. Entin-Wohlman and Z. Ovadyahu, Phys. Rev. B 14, 1002 (1976).

84. J. P. Garno, J. App1. Phys. 48, 4627 (1977). 